\title{
SMEFTsim 3.0 - a practical guide
}

\author{
Ilaria Brivio \\ Institut für Theoretische Physik, Universität Heidelberg. \\ Philosophenweg 16, 69120 Heidelberg, Germany \\ E-mail: brivio@thphys.uni-heidelberg.de
}

ABSTRACT: The SMEFTsim package [1] is designed to enable automated computations in the Standard Model Effective Field Theory (SMEFT), where the SM Lagrangian is extended with a complete basis of dimension six operators. It contains a set of models written in FeynRules and pre-exported to the UFO format, for usage within Monte Carlo event generators. The models differ in the flavor assumptions and in the input parameters chosen for the electroweak sector. The present document provides a self-contained, pedagogical reference that collects all the theoretical and technical aspects relevant to the use of SMEFTsim and it documents the release of version 3.0. Compared to the previous release, the description of Higgs production via gluon-fusion in the SM has been significantly improved, two flavor assumptions for studies in the top quark sector have been added, and a new feature has been implemented, that enables the treatment of linearized SMEFT corrections to the propagators of unstable particles.

SMEFTsim 3.0 is available on the Github website https://SMEFTsim.github.io and on the FeynRules database http://feynrules.irmp.ucl.ac.be/wiki/SMEFT.

Keywords: Beyond Standard Model, Effective Field Theories, Higgs Physics

ARXiv EPrint: 2012.11343 


\section{Contents}

1 Introduction 1

1.1 Basics and notation 3

2 EWSB, field and parameter redefinitions $\quad 6$

2.1 Higgs sector 6

$\begin{array}{ll}2.2 \text { Gauge sector } & 7\end{array}$

3 Flavor assumptions $\quad 9$

$\begin{array}{lr}3.1 \text { general: general flavor structure } & 10\end{array}$

$\begin{array}{lll}3.2 & \text { U35: maximal U }(3)^{5} \text { symmetry } & 12\end{array}$

$\begin{array}{lll}3.3 & \text { MFV: linear minimal flavor violation } & 14\end{array}$

3.4 top, topU31: $\mathrm{U}(2)^{3}$ symmetry in the quark sector 18

$\begin{array}{ll}3.5 & \text { Comparison with the literature } \\ \end{array}$

4 Input parameters $\quad 27$

4.1 Implementation in SMEFTsim 30

4.2 Higgs and EW sectors 31

4.2.1 $\left\{\alpha_{\mathrm{em}}, m_{Z}, G_{F}\right\}$ scheme $\quad 32$

4.2.2 $\left\{m_{W}, m_{Z}, G_{F}\right\}$ scheme 34

$\begin{array}{lll}4.3 & \text { Yukawa sector } & 35\end{array}$

5 SM loop-generated Higgs interactions $\quad 36$

$\begin{array}{lll}5.1 & \text { Validity of the approximations used } & 38\end{array}$

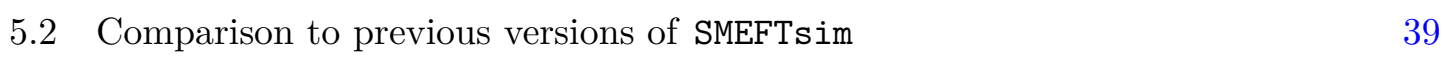

6 Propagator corrections $\quad 40$

6.1 Implementation in SMEFTsim 41

7 Usage in Mathematica $\quad 44$

8 Usage in MadGraph5_aMC@NLO $\quad 49$

$\begin{array}{ll}8.1 \text { Parameter cards and restrictions } & 50\end{array}$

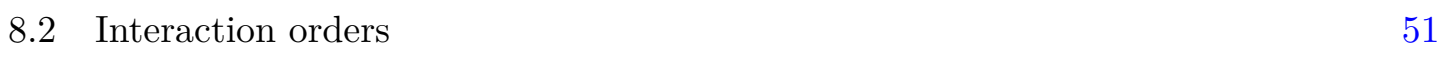

$\begin{array}{lll}\text { 8.2.1 Definitions } & 52\end{array}$

$\begin{array}{lll}\text { 8.2.2 Recommended use } & 53\end{array}$

$\begin{array}{lll}\text { 8.3 Propagator corrections and decay widths } & 54\end{array}$

8.3.1 Method (a): linearized corrections $\quad 54$

8.3.2 Method (b): full corrections $\quad 55$

8.4 Example: Higgs production and decay including $W, Z$ propagator corrections 56

$\begin{array}{lll}\text { 8.4.1 STXS for } \bar{q} q \rightarrow h \bar{q} q & 56\end{array}$

$\begin{array}{lll}\text { 8.4.2 } h \rightarrow e^{+} e^{-} \mu^{+} \mu^{-} & 58\end{array}$ 
A Analytic expressions of decay width corrections 59

$\begin{array}{lll}\text { A.1 } Z \text { boson } & 59\end{array}$

A.2 $W$ boson 61

A.3 Higgs boson 62

A.4 Top quark 63

B What's new in version 3.0 $\quad 64$

C Conversion tables between flavor assumptions $\quad 64$

$\begin{array}{ll}\text { D Parameter definitions in the code implementation } & 71\end{array}$

E Comparison to other SMEFT UFO models $\quad 79$

$\begin{array}{lll}\text { E.1 dim6top } & 79\end{array}$

E.2 SMEFT@NLO 80

$\begin{array}{lll}\text { F Validation of the UFO models } & 89\end{array}$

\section{Introduction}

LHC physics is about to enter a precision era that will span over the next two decades. During this time, new opportunities to hunt for new physics will arise: direct searches of new particles will be complemented by indirect searches, that target possible deviations from the predictions of the Standard Model (SM). While the isolation of this kind of signatures is not without challenges, indirect searches present some very attractive features. Most notably, they do not rely on specific assumptions about the nature of the new physics under scrutiny and, at the same time, their sensitivity in terms of new physics scales can potentially extend beyond the energy reach of the collider.

The Standard Model Effective Field Theory (SMEFT) is the best established theory framework to describe such effects. Its formulation employs the degrees of freedom and gauge symmetries of the SM and it is structured as an infinite series of operators sorted by canonical dimension. At the observables level, it reproduces a series expansion in $(E / \Lambda)$, being $E$ the typical energy exchanged in a process and $\Lambda$ the mass scale that characterizes the beyond-SM (BSM) dynamics. The condition $(E / \Lambda) \ll 1$, indicating the near decoupling of the new physics sector, is necessarily assumed.

The SMEFT has been developed extensively in the past ten years, laying the ground for a systematic program for indirect searches [2-4]. The ultimate goal is to measure as many EFT parameters as possible, in a manner that enables the extraction of unbiased information about the underlying physics. The crucial aspect of this program is its transversality: the SMEFT contains a large number of parameters, each typically entering the description 
of several processes. Combining measurements of different observables is then mandatory in order to preserve the model-independence of the analysis. To date, this principle has been applied within individual sectors as well as across Higgs, electroweak (EW) and top quark measurements, see refs. [5-15] for recent examples. The incorporation of data from flavor observables (including non-LHC experiments) would be very valuable in this context, as most of the SMEFT parameter space is "flavorful". First steps in this direction were taken in [16-19].

The theory developments have been accompanied by the publication of a number of computing tools that automate most stages of a SMEFT study [20]. These include the definition of non-redundant operator bases and the translation between them [21-24], the matching to concrete BSM models or to the low-energy EFT and the renormalization group running [25-31], the extraction of the Feynman rules in $R_{\xi}$ gauges [32, 33] and in the background field gauge [34], Monte Carlo simulations [1, 35-37] and global analyses [13, 38-41].

The SMEFTsim package [1] was designed in order to enable the Monte Carlo simulation of arbitrary processes in the effective theory, in the spirit of providing a unified, generalpurpose tool for SMEFT physics at the LHC. It provides complete tree level, unitary gauge predictions at $\mathcal{O}\left(\Lambda^{-2}\right)$, including all the dimension six operators in the so-called Warsaw basis [42]. The field and parameter redefinitions that are required in order to compute physical observables in the SMEFT are conveniently performed internally. The package contains FeynRules $[43,44]$ source files and a set of models pre-exported to the UFO format [45]. Although the latter are in principle compatible with most Monte Carlo generators, they have been optimized for the use in MadGraph5_aMC@NLO [46], that is illustrated in section 8. Potential issues due, for instance, to the event generation entering regions where the EFT validity or the unitarity of the $S$-matrix are violated are not addressed within SMEFTsim itself, but can be generally treated with tools offered by the Monte Carlo generators or with theoretical assessments a posteriori.

The UFO models differ in the flavor assumptions and in the choice of the input parameters for the EW sector. ${ }^{1}$ The original release implemented three alternative flavor scenarios: a general one, a $\mathrm{U}(3)^{5}$-symmetric case and a linear minimal flavor violation (MFV) option where BSM CP-violating phases are forbidden. For each setup, it offered two EW input sets: $\left\{\alpha_{\mathrm{em}}, m_{Z}, G_{F}\right\}$ or $\left\{m_{W}, m_{Z}, G_{F}\right\}$.

This work documents the release of SMEFTsim version 3.0, that introduces a number of improvements summarized below. It is also meant as a pedagogical and self-contained reference for its usage, where all the relevant theoretical aspects are reviewed in detail.

The present document is structured as follows: sections 1-4 review the theoretical background while sections 5-8 describe technical aspects of the FeynRules and UFO implementations and provide recommendations for their use.

The notation is fixed in section 1.1. Section 2 focuses on the bosonic sector and it reviews the field and parameter redefinitions required to ensure a canonical parameterization of the kinetic terms and scalar potential. Section 3 is devoted to the flavor structure of

\footnotetext{
${ }^{1}$ The original release contained two fully equivalent implementations, that were called model sets A and B. Both were provided for debugging and cross-validation. Set B is not supported anymore starting from version 3.0, which is based on set $\mathrm{A}$.
} 
the SMEFT and it defines the five scenarios implemented in SMEFTsim. Some significant changes have been made compared to version 2, that are documented in detail, and two new flavor options have been introduced (top, topU31) that comply with the recommendations for studies of top quark observables [36]. Section 4 provides a general discussion of how the extraction of numerical values for the SM parameters is affected in the presence of higher-dimensional operators, and illustrates the treatment of these effects in SMEFTsim.

Section 5 documents the implementation of Higgs interactions that are purely loopgenerated in the SM, namely $h \gamma \gamma, h Z \gamma, h g g$ : as SMEFTsim only supports tree-level interactions, these are treated as effective vertices in the large top mass limit, which is a good approximation for Higgs production and decay processes. Compared to version 2, the description of Higgs-gluon vertices has been substantially improved, such that it can now model one-loop SM interactions with up to 4 gluons. Section 6 focuses on SMEFT effects in the propagators of unstable particles, that arise due to modifications of their pole masses or decay widths. A new feature has been introduced in version 3.0, that enables the inclusion of such effects, linearized in the EFT parameters, in Monte Carlo simulations. To our knowledge SMEFTsim is the first publicly available UFO model to implement such a tool. Sections 7, 8 provide recommendations for the use of SMEFTsim in Mathematica and in MadGraph5_aMC@NLO respectively, and in section 9 we conclude.

Additional useful material is provided in the appendices: analytic expressions of the decay widths implemented in the propagator corrections (appendix A), a list of changes made in version 3.0 (appendix B), tables to facilitate the conversion between flavor assumptions (appendix C), between theory and code notation (appendix D) and between SMEFTsim and dim6top or SMEFT@NLO (appendix E). Finally, appendix F documents the validation of the UFO models, that followed the procedure recommended in [47].

\subsection{Basics and notation}

We consider the SMEFT Lagrangian truncated at the dimension-6 level:

$$
\mathcal{L}_{\mathrm{SMEFT}}=\mathcal{L}_{\mathrm{SM}}+\mathcal{L}_{6}
$$

We neglect all lepton- and baryon-number violating terms, which includes the dimension-5 Weinberg operator that generates a Majorana mass term for neutrinos. For future convenience, the SM Lagrangian is split into four terms:

$$
\mathcal{L}_{\mathrm{SM}}=\mathcal{L}_{\text {gauge }}+\mathcal{L}_{\text {fermions }}+\mathcal{L}_{\text {Yukawa }}+\mathcal{L}_{\text {Higgs }}
$$

where

$$
\begin{aligned}
\mathcal{L}_{\text {gauge }} & =-\frac{1}{4} B_{\mu \nu} B^{\mu \nu}-\frac{1}{4} W_{\mu \nu}^{i} W^{i \mu \nu}-\frac{1}{4} G_{\mu \nu}^{a} G^{a \mu \nu}, \\
\mathcal{L}_{\text {fermions }} & =\bar{q} i \not D q+\bar{u} i \not D u+\bar{d} i \not D d+\bar{l} i \not D l+\bar{e} i \not D e \\
\mathcal{L}_{\text {Yukawa }} & =-\bar{d} Y_{d} H^{\dagger} q-\bar{u} Y_{u} \tilde{H}^{\dagger} q-\bar{e} Y_{l} H^{\dagger} l+\text { h.c. } \\
\mathcal{L}_{\text {Higgs }} & =D_{\mu} H^{\dagger} D^{\mu} H+m^{2}\left(H^{\dagger} H\right)-\lambda\left(H^{\dagger} H\right)^{2} .
\end{aligned}
$$


$q, l$ represent the left-handed quark and lepton doublets respectively, and $u, d, e$ the righthanded quarks and leptons. $H$ is the Higgs doublet and $\tilde{H}=i \sigma^{2} H^{*}$, where $\sigma^{i}, i=\{1,2,3\}$ are the Pauli matrices. $Y_{d}, Y_{u}, Y_{l}$ are the $3 \times 3$ Yukawa matrices of the down and up quarks and of the charged leptons. Covariant derivatives are defined with a plus sign, i.e. ${ }^{2}$

$$
D_{\mu} q=\left[\partial_{\mu}+i g_{s} T^{a} G_{\mu}^{a}+i \frac{g_{W}}{2} \sigma^{i} W_{\mu}^{i}+i \mathbf{y}_{q} g_{1} B_{\mu}\right] q
$$

$T^{a} \equiv \lambda^{a} / 2, a=\{1, \ldots, 8\}$ are the $\mathrm{SU}(3)_{c}$ generators, with $\lambda^{a}$ the Gell-Mann matrices. $\mathbf{y}_{q}=1 / 6$ denotes the hypercharge of the $q$ field and $g_{s}, g_{W}, g_{1}$ are the $\mathrm{SU}(3)_{c} \times \mathrm{SU}(2)_{L} \times$ $\mathrm{U}(1)_{Y}$ coupling constants. As a general rule, color indices are denoted by $a, b, c, d, \mathrm{SU}(2)_{L}$ indices by $i, j, k$ and flavor indices by $p, r, s, t$. Summation over identical indices is always understood, unless otherwise specified.

The Lagrangian $\mathcal{L}_{6}$ contains a complete and non-redundant basis of dimension-6 operators $Q_{\alpha}$ constructed with the SM fields and invariant under the $\mathrm{SU}(3)_{c} \times \mathrm{U}(2)_{L} \times \mathrm{U}(1)_{Y}$ gauge symmetry. SMEFTsim implements the Warsaw basis [42], whose operators are collected in 8 groups, following the classification of ref. [48]. Class 8 is further split into 4 subgroups: ${ }^{3}$

$$
\begin{aligned}
\mathcal{L}_{6} & =\mathcal{L}_{6}^{(1)}+\mathcal{L}_{6}^{(2)}+\mathcal{L}_{6}^{(3)}+\mathcal{L}_{6}^{(4)}+\mathcal{L}_{6}^{(7)}+\mathcal{L}_{6}^{(8)}+\left[\mathcal{L}_{6}^{(5)}+\mathcal{L}_{6}^{(6)}+\text { h.c. }\right] \\
\mathcal{L}_{6}^{(8)} & =\mathcal{L}_{6}^{(8 a)}+\mathcal{L}_{6}^{(8 b)}+\mathcal{L}_{6}^{(8 c)}+\left[\mathcal{L}_{6}^{(8 d)}+\text { h.c. }\right] .
\end{aligned}
$$

Each sub-Lagrangian has the form

$$
\mathcal{L}_{6}^{(n)}=\frac{1}{\Lambda^{2}} \sum_{\alpha} C_{\alpha} Q_{\alpha}
$$

with the sum running over the class- $n$ operators $\left\{Q_{\alpha}\right\}$ defined in table 1 and $C_{\alpha}$ denoting the associated Wilson coefficients. Both $Q_{\alpha}$ and $C_{\alpha}$ generally carry flavor indices, that are implicitly contracted in eq. (1.10). In this basis, explicit $\mathrm{CP}$ violation is carried by the real coefficients $C_{\widetilde{G}}, C_{\widetilde{W}}, C_{H \widetilde{G}}, C_{H \widetilde{W}}, C_{H \widetilde{B}}, C_{H \widetilde{W} B}$ and by the imaginary parts of the Wilson coefficients associated to non-Hermitian fermionic operators, namely those in $\mathcal{L}_{6}^{(5),(6),(8 d)}$ and $\mathcal{O}_{H u d}$. Baryon-number violating operators are omitted.

The operators definitions use the following notation:

$$
\begin{aligned}
\widetilde{X}^{\mu \nu} & =\frac{1}{2} \varepsilon^{\mu \nu \rho \sigma} X_{\rho \sigma}, & & H^{\dagger} i \overleftrightarrow{D}_{\mu} H=H^{\dagger}\left(i D_{\mu} H\right)-\left(i D_{\mu} H^{\dagger}\right) H, \\
\sigma^{\mu \nu} & =\frac{i}{2}\left[\gamma^{\mu}, \gamma^{\nu}\right], & H^{\dagger} i \overleftrightarrow{D}_{\mu}^{i} H & =H^{\dagger} \sigma^{i}\left(i D_{\mu} H\right)-\left(i D_{\mu} H^{\dagger}\right) \sigma^{i} H
\end{aligned}
$$

\footnotetext{
${ }^{2}$ The covariant derivative sign is handled automatically by FeynRules. The convention chosen here also implies that gauge field strenghts have the form $W_{\mu \nu}^{i}=\partial_{\mu} W_{\nu}^{i}-\partial_{\nu} W_{\mu}^{i}-g_{W} \varepsilon^{i j k} W_{\mu}^{j} W_{\nu}^{k}$, etc.

${ }^{3}$ Note that $\mathcal{L}_{6}^{(7)}$ implicitly contains $\left(Q_{H u d}+\right.$ h.c. $)$, as this operator is not Hermitian.
} 


\begin{tabular}{|c|c|c|c|c|c|}
\hline \multicolumn{2}{|r|}{$\mathcal{L}_{6}^{(1)}-X^{3}$} & \multicolumn{2}{|r|}{$\mathcal{L}_{6}^{(6)}-\psi^{2} X H$} & \multicolumn{2}{|r|}{$\mathcal{L}_{6}^{(8 b)}-(\bar{R} R)(\bar{R} R)$} \\
\hline$Q_{G}$ & $f^{a b c} G_{\mu}^{a \nu} G_{\nu}^{b \rho} G_{\rho}^{c \mu}$ & $Q_{e W}$ & $\left(\bar{l}_{p} \sigma^{\mu \nu} e_{r}\right) \sigma^{i} H W_{\mu \nu}^{i}$ & $Q_{e e}$ & $\left(\bar{e}_{p} \gamma_{\mu} e_{r}\right)\left(\bar{e}_{s} \gamma^{\mu} e_{t}\right)$ \\
\hline$Q_{\widetilde{G}}$ & $f^{a b c} \widetilde{G}_{\mu}^{a \nu} G_{\nu}^{b \rho} G_{\rho}^{c \mu}$ & $Q_{e B}$ & $\left(\bar{l}_{p} \sigma^{\mu \nu} e_{r}\right) H B_{\mu \nu}$ & $Q_{u u}$ & $\left(\bar{u}_{p} \gamma_{\mu} u_{r}\right)\left(\bar{u}_{s} \gamma^{\mu} u_{t}\right)$ \\
\hline$Q_{W}$ & $\varepsilon^{i j k} W_{\mu}^{i \nu} W_{\nu}^{j \rho} W_{\rho}^{k \mu}$ & $Q_{u G}$ & $\left(\bar{q}_{p} \sigma^{\mu \nu} T^{a} u_{r}\right) \widetilde{H} G_{\mu \nu}^{a}$ & $Q_{d d}$ & $\left(\bar{d}_{p} \gamma_{\mu} d_{r}\right)\left(\bar{d}_{s} \gamma^{\mu} d_{t}\right)$ \\
\hline$Q_{\widetilde{W}}$ & $\varepsilon^{i j k} \widetilde{W}_{\mu}^{i \nu} W_{\nu}^{j \rho} W_{\rho}^{k \mu}$ & $Q_{u W}$ & $\left(\bar{q}_{p} \sigma^{\mu \nu} u_{r}\right) \sigma^{i} \widetilde{H} W_{\mu \nu}^{i}$ & $Q_{e u}$ & $\left(\bar{e}_{p} \gamma_{\mu} e_{r}\right)\left(\bar{u}_{s} \gamma^{\mu} u_{t}\right)$ \\
\hline \multicolumn{2}{|r|}{$\mathcal{L}_{6}^{(2)}-H^{6}$} & $Q_{u B}$ & $\left(\bar{q}_{p} \sigma^{\mu \nu} u_{r}\right) \widetilde{H} B_{\mu \nu}$ & $Q_{e d}$ & $\left(\bar{e}_{p} \gamma_{\mu} e_{r}\right)\left(\bar{d}_{s} \gamma^{\mu} d_{t}\right)$ \\
\hline$Q_{H}$ & $\left(H^{\dagger} H\right)^{3}$ & $Q_{d G}$ & $\left(\bar{q}_{p} \sigma^{\mu \nu} T^{a} d_{r}\right) H G_{\mu \nu}^{a}$ & $Q_{u d}^{(1)}$ & $\left(\bar{u}_{p} \gamma_{\mu} u_{r}\right)\left(\bar{d}_{s} \gamma^{\mu} d_{t}\right)$ \\
\hline \multicolumn{2}{|r|}{$\mathcal{L}_{6}^{(3)}-H^{4} D^{2}$} & $Q_{d W}$ & $\left(\bar{q}_{p} \sigma^{\mu \nu} d_{r}\right) \sigma^{i} H W_{\mu \nu}^{i}$ & $Q_{u d}^{(8)}$ & $\left(\bar{u}_{p} \gamma_{\mu} T^{a} u_{r}\right)\left(\bar{d}_{s} \gamma^{\mu} T^{a} d_{t}\right)$ \\
\hline $\begin{array}{l}Q_{H \square} \\
Q_{H D}\end{array}$ & $\begin{array}{l}\left(H^{\dagger} H\right) \square\left(H^{\dagger} H\right) \\
\left(D^{\mu} H^{\dagger} H\right)\left(H^{\dagger} D_{\mu} H\right)\end{array}$ & $Q_{d B}$ & $\left(\bar{q}_{p} \sigma^{\mu \nu} d_{r}\right) H B_{\mu \nu}$ & & \\
\hline \multicolumn{2}{|r|}{$\mathcal{L}_{6}^{(4)}-X^{2} H^{2}$} & \multicolumn{2}{|r|}{$\mathcal{L}_{6}^{(7)}-\psi^{2} H^{2} D$} & \multicolumn{2}{|r|}{$\mathcal{L}_{6}^{(8 c)}-(\bar{L} L)(\bar{R} R)$} \\
\hline$Q_{H G}$ & $H^{\dagger} H G_{\mu \nu}^{a} G^{a \mu \nu}$ & $Q_{H l}^{(1)}$ & $\left(H^{\dagger} i \overleftrightarrow{D}_{\mu} H\right)\left(\bar{l}_{p} \gamma^{\mu} l_{r}\right)$ & $Q_{l e}$ & $\left(\bar{l}_{p} \gamma_{\mu} l_{r}\right)\left(\bar{e}_{s} \gamma^{\mu} e_{t}\right)$ \\
\hline$Q_{H \widetilde{G}}$ & $H^{\dagger} H \widetilde{G}_{\mu \nu}^{a} G^{a \mu \nu}$ & $Q_{H l}^{(3)}$ & $\left(H^{\dagger} i \overleftrightarrow{D}_{\mu}^{i} H\right)\left(\bar{l}_{p} \sigma^{i} \gamma^{\mu} l_{r}\right)$ & $Q_{l u}$ & $\left(\bar{l}_{p} \gamma_{\mu} l_{r}\right)\left(\bar{u}_{s} \gamma^{\mu} u_{t}\right)$ \\
\hline$Q_{H W}$ & $H^{\dagger} H W_{\mu \nu}^{i} W^{I \mu \nu}$ & $Q_{\mathrm{He}}$ & $\left(H^{\dagger} i \overleftrightarrow{D}_{\mu} H\right)\left(\bar{e}_{p} \gamma^{\mu} e_{r}\right)$ & $Q_{l d}$ & $\left(\bar{l}_{p} \gamma_{\mu} l_{r}\right)\left(\bar{d}_{s} \gamma^{\mu} d_{t}\right)$ \\
\hline$Q_{H \widetilde{W}}$ & $H^{\dagger} H \widetilde{W}_{\mu \nu}^{i} W^{i \mu \nu}$ & $Q_{H q}^{(1)}$ & $\left(H^{\dagger} i \overleftrightarrow{D}_{\mu} H\right)\left(\bar{q}_{p} \gamma^{\mu} q_{r}\right)$ & $Q_{q e}$ & $\left(\bar{q}_{p} \gamma_{\mu} q_{r}\right)\left(\bar{e}_{s} \gamma^{\mu} e_{t}\right)$ \\
\hline$Q_{H B}$ & $H^{\dagger} H B_{\mu \nu} B^{\mu \nu}$ & $Q_{H q}^{(3)}$ & $\left(H^{\dagger} i \overleftrightarrow{D}_{\mu}^{i} H\right)\left(\bar{q}_{p} \sigma^{i} \gamma^{\mu} q_{r}\right)$ & $Q_{q u}^{(1)}$ & $\left(\bar{q}_{p} \gamma_{\mu} q_{r}\right)\left(\bar{u}_{s} \gamma^{\mu} u_{t}\right)$ \\
\hline$Q_{H \widetilde{B}}$ & $H^{\dagger} H \widetilde{B}_{\mu \nu} B^{\mu \nu}$ & $Q_{H u}$ & $\left(H^{\dagger} i \overleftrightarrow{D}_{\mu} H\right)\left(\bar{u}_{p} \gamma^{\mu} u_{r}\right)$ & $Q_{q u}^{(8)}$ & $\left(\bar{q}_{p} \gamma_{\mu} T^{a} q_{r}\right)\left(\bar{u}_{s} \gamma^{\mu} T^{a} u_{t}\right)$ \\
\hline$Q_{H W B}$ & $H^{\dagger} \sigma^{i} H W_{\mu \nu}^{i} B^{\mu \nu}$ & $Q_{H d}$ & $\left(H^{\dagger} i \overleftrightarrow{D}_{\mu} H\right)\left(\bar{d}_{p} \gamma^{\mu} d_{r}\right)$ & $Q_{q d}^{(1)}$ & $\left(\bar{q}_{p} \gamma_{\mu} q_{r}\right)\left(\bar{d}_{s} \gamma^{\mu} d_{t}\right)$ \\
\hline$Q_{H \widetilde{W} B}$ & $H^{\dagger} \sigma^{i} H \widetilde{W}_{\mu \nu}^{i} B^{\mu \nu}$ & $Q_{H u d}+$ h.c. & $i\left(\widetilde{H}^{\dagger} D_{\mu} H\right)\left(\bar{u}_{p} \gamma^{\mu} d_{r}\right)$ & $Q_{q d}^{(8)}$ & $\left(\bar{q}_{p} \gamma_{\mu} T^{a} q_{r}\right)\left(\bar{d}_{s} \gamma^{\mu} T^{a} d_{t}\right)$ \\
\hline \multicolumn{2}{|r|}{$\mathcal{L}_{6}^{(5)}-\psi^{2} H^{3}$} & \multicolumn{2}{|c|}{$\mathcal{L}_{6}^{(8 a)}-(\bar{L} L)(\bar{L} L)$} & \multicolumn{2}{|c|}{$\mathcal{L}_{6}^{(8 d)}-(\bar{L} R)(\bar{R} L),(\bar{L} R)(\bar{L} R)$} \\
\hline$Q_{e H}$ & $\left(H^{\dagger} H\right)\left(\bar{l}_{p} e_{r} H\right)$ & $Q_{l l}$ & $\left(\bar{l}_{p} \gamma_{\mu} l_{r}\right)\left(\bar{l}_{s} \gamma^{\mu} l_{t}\right)$ & $Q_{l e d q}$ & $\left(\bar{l}_{p}^{j} e_{r}\right)\left(\bar{d}_{s} q_{t j}\right)$ \\
\hline$Q_{u H}$ & $\left(H^{\dagger} H\right)\left(\bar{q}_{p} u_{r} \widetilde{H}\right)$ & $Q_{q q}^{(1)}$ & $\left(\bar{q}_{p} \gamma_{\mu} q_{r}\right)\left(\bar{q}_{s} \gamma^{\mu} q_{t}\right)$ & $Q_{q u q d}^{(1)}$ & $\left(\bar{q}_{p}^{j} u_{r}\right) \varepsilon_{j k}\left(\bar{q}_{s}^{k} d_{t}\right)$ \\
\hline \multirow[t]{3}{*}{$Q_{d H}$} & $\left(H^{\dagger} H\right)\left(\bar{q}_{p} d_{r} H\right)$ & $Q_{q q}^{(3)}$ & $\left(\bar{q}_{p} \gamma_{\mu} \sigma^{i} q_{r}\right)\left(\bar{q}_{s} \gamma^{\mu} \sigma^{i} q_{t}\right)$ & $Q_{q u q d}^{(8)}$ & $\left(\bar{q}_{p}^{j} T^{a} u_{r}\right) \varepsilon_{j k}\left(\bar{q}_{s}^{k} T^{a} d_{t}\right)$ \\
\hline & & $Q_{l q}^{(1)}$ & $\left(\bar{l}_{p} \gamma_{\mu} l_{r}\right)\left(\bar{q}_{s} \gamma^{\mu} q_{t}\right)$ & $Q_{l e q u}^{(1)}$ & $\left(\bar{l}_{p}^{j} e_{r}\right) \varepsilon_{j k}\left(\bar{q}_{s}^{k} u_{t}\right)$ \\
\hline & & $Q_{l q}^{(3)}$ & $\left(\bar{l}_{p} \gamma_{\mu} \sigma^{i} l_{r}\right)\left(\bar{q}_{s} \gamma^{\mu} \sigma^{i} q_{t}\right)$ & $Q_{l e q u}^{(3)}$ & $\left(\bar{l}_{p}^{j} \sigma_{\mu \nu} e_{r}\right) \varepsilon_{j k}\left(\bar{q}_{s}^{k} \sigma^{\mu \nu} u_{t}\right)$ \\
\hline
\end{tabular}

Table 1. $\mathcal{L}_{6}$ operators in the Warsaw basis [42], categorized into eight classes $\mathcal{L}_{6}^{(n)}$ as in [48]. Only baryon number-conserving invariants are retained. The flavor indices $p, r, s, t$ are suppressed in the operators' names. 


\section{EWSB, field and parameter redefinitions}

This section reviews the Lagrangian manipulations that are required in order to compute physical processes in the SMEFT truncated at $\mathcal{O}\left(\Lambda^{-2}\right) .{ }^{4}$ The procedure described here largely overlaps with what reported e.g. in $[1,32,48-59]$.

\subsection{Higgs sector}

The operator $Q_{H}$ introduces a perturbation of the Higgs potential:

$$
V(H)=-m^{2} H^{\dagger} H+\lambda\left(H^{\dagger} H\right)^{2}-\frac{C_{H}}{\Lambda^{2}}\left(H^{\dagger} H\right)^{3} .
$$

The true minimum of the potential, that triggers the electroweak symmetry breaking, is

$$
\begin{aligned}
\left\langle H^{\dagger} H\right\rangle=\frac{v_{T}^{2}}{2} & \equiv \frac{v^{2}}{2}\left[1+\frac{3}{4 \lambda} \frac{v^{2}}{\Lambda^{2}} C_{H}\right]+\mathcal{O}\left(\Lambda^{-4}\right) \\
& =\frac{v^{2}}{2}\left[1+\frac{3}{4 \lambda} \bar{C}_{H}\right]+\mathcal{O}\left(\Lambda^{-4}\right),
\end{aligned}
$$

with

$$
v^{2}=\frac{m^{2}}{2 \lambda}
$$

We have introduced the "bar" notation for Wilson coefficients:

$$
\bar{C}_{\alpha} \equiv \frac{v_{T}^{2}}{\Lambda^{2}} C_{\alpha} .
$$

Note that because $v=v_{T}+\mathcal{O}\left(\Lambda^{-2}\right)$ and $\mathcal{O}\left(\Lambda^{-4}\right)$ contributions are entirely neglected, the two quantities $v, v_{T}$ are interchangeable whenever they multiply a Wilson coefficent. ${ }^{5}$

The Higgs field $H$ is expanded around its vacuum expectation value (vev) as

$$
H=\left(\begin{array}{c}
-i G^{+} \\
\frac{v_{T}+h+i G^{0}}{\sqrt{2}}
\end{array}\right),
$$

with $G^{+}, G^{0}$ the charged and neutral Goldstone bosons and $h$ the physical Higgs boson. In the broken phase, the kinetic terms of the scalar fields receive corrections from the operators $Q_{H \square}, Q_{H D}$. As the scope of SMEFTsim is limited to tree-level calculations, we choose to work in unitary gauge and neglect EFT effects in the Goldstone sector, both in the present discussion and in the code implementations. The Goldstone bosons case and the generalization of the gauge fixing procedure in the SMEFT were addressed in refs. [3, 32, 58-62].

\footnotetext{
${ }^{4}$ Within a Monte Carlo event generation, SMEFTsim generally enables the computation of higher order corrections to a given observable, such as $\mathcal{O}\left(\Lambda^{-4}\right)$ corrections stemming from the square of $\mathcal{O}\left(\Lambda^{-2}\right)$ amplitudes (see section 8). However, consistent results are only provided to $\mathcal{O}\left(\Lambda^{-2}\right)$, as the SMEFT Lagrangian implemented is truncated at this order.

${ }^{5}$ In fact, it would be more appropriate to define the $\bar{C}_{\alpha}$ notation with the parameter $\hat{v}$ defined in section 4.2 , rather than with $v_{T}$. However, as long as $\mathcal{O}\left(\Lambda^{-4}\right)$ terms are neglected, all three $v$ 's are formally identical when multiplying a Wilson coefficient.
} 
Using integration by parts, the kinetic term of the physical Higgs boson takes the form

$$
\mathcal{L}_{\text {Higgs }}+\mathcal{L}_{6}=\frac{1}{2} \partial_{\mu} h \partial^{\mu} h\left[1-2 \Delta \kappa_{H}\right]+\ldots \quad \text { with } \quad \Delta \kappa_{H}=\bar{C}_{H \square}-\frac{\bar{C}_{H D}}{4},
$$

and it is brought to the canonical normalization via the field redefinition

$$
h \rightarrow\left[1+\Delta \kappa_{H}\right] h .
$$

This replacement is formally operated on the entire $\mathcal{L}_{\text {SMEFT }}$. However, when applied to $\mathcal{L}_{6}$, its net effect is of $\mathcal{O}\left(\Lambda^{-4}\right)$. As we work at $\mathcal{O}\left(\Lambda^{-2}\right)$, the replacement only needs to be performed on $\mathcal{L}_{\mathrm{SM}}$. This holds for all field and parameter redefinitions introduced in the following, unless otherwise specified. For the same reason, all quantities in a Wilson coefficient's prefactor are understood to be defined in the SM limit.

The main consequence of (2.7) is that the Wilson coefficients $C_{H \square}, C_{H D}$ are recast into an overall rescaling of all SM Higgs couplings. The resulting Higgs potential is

$$
\begin{aligned}
V(H)= & h^{2} \lambda v_{T}^{2}\left[1+2 \Delta \kappa_{H}-\frac{3}{2 \lambda} \bar{C}_{H}\right]+h^{3} \lambda v_{T}\left[1+3 \Delta \kappa_{H}-\frac{5}{2 \lambda} \bar{C}_{H}\right] \\
& +h^{4} \frac{\lambda}{4}\left[1+4 \Delta \kappa_{H}-\frac{15}{2 \lambda} \bar{C}_{H}\right]-\frac{3}{4} \frac{h^{5}}{v_{T}} \bar{C}_{H}-\frac{1}{8} \frac{h^{6}}{v_{T}^{2}} \bar{C}_{H} .
\end{aligned}
$$

In the FeynRules implementation, the redefinitions of the physical Higgs field, eq. (2.7), and of the vev, eq. (2.2), are embedded in the definition of the Higgs doublet.

\subsection{Gauge sector}

Upon EWSB, the operators $Q_{H G}, Q_{H W}, Q_{H B}, Q_{H W B}$ induce corrections to the kinetic terms of the gauge bosons. The first three lead to overall rescalings:

$$
\mathcal{L}_{\text {gauge }}+\mathcal{L}_{6}=-\frac{1}{4} B_{\mu \nu} B^{\mu \nu}\left[1-2 \bar{C}_{H B}\right]-\frac{1}{4} W_{\mu \nu}^{i} W^{i \mu \nu}\left[1-2 \bar{C}_{H W}\right]-\frac{1}{4} G_{\mu \nu}^{a} G^{a \mu \nu}\left[1-2 \bar{C}_{H G}\right]+\ldots
$$

where the dots stand for all other interaction terms induced by $\mathcal{L}_{6}$. The canonical normalization is easily restored at $\mathcal{O}\left(\Lambda^{-2}\right)$, via the field redefinitions

$$
G_{\mu}^{a} \rightarrow G_{\mu}^{a}\left(1+\bar{C}_{H G}\right), \quad W_{\mu}^{i} \rightarrow W_{\mu}^{i}\left(1+\bar{C}_{H W}\right), \quad B_{\mu} \rightarrow B_{\mu}\left(1+\bar{C}_{H B}\right) .
$$

In order to leave the covariant derivatives unchanged, the coupling constants need to be redefined at the same time. Neglecting $\mathcal{O}\left(\Lambda^{-4}\right)$ corrections:

$$
g_{s} \rightarrow g_{s}\left(1-\bar{C}_{H G}\right), \quad g_{W} \rightarrow g_{W}\left(1-\bar{C}_{H W}\right), \quad g_{1} \rightarrow g_{1}\left(1-\bar{C}_{H B}\right) .
$$

The operator $Q_{H W B}$ introduces a kinetic mixing between the $B$ and $W^{3}$ fields of the form

$$
-\frac{C_{H W B}}{2} \frac{v_{T}^{2}}{\Lambda^{2}} W_{\mu \nu}^{3} B^{\mu \nu}
$$

The rotation [48]

$$
\left(\begin{array}{c}
W_{\mu}^{3} \\
B_{\mu}
\end{array}\right) \rightarrow\left(\begin{array}{cc}
1 & -\bar{C}_{H W B} / 2 \\
-\bar{C}_{H W B} / 2 & 1
\end{array}\right)\left(\begin{array}{c}
W_{\mu}^{3} \\
B_{\mu}
\end{array}\right)
$$


removes this residual mixing and leads to fully canonical and diagonal kinetic terms. Once eqs. (2.10), (2.11), (2.13) have been applied, the electric-charge eigenstates $W^{ \pm}$are obtained via the usual rotation

$$
\left(\begin{array}{l}
W_{\mu}^{1} \\
W_{\mu}^{2}
\end{array}\right)=\frac{1}{\sqrt{2}}\left(\begin{array}{cc}
1 & 1 \\
i & -i
\end{array}\right)\left(\begin{array}{l}
W_{\mu}^{+} \\
W_{\mu}^{-}
\end{array}\right),
$$

while the mass matrix of the neutral bosons is diagonalized by

$$
\left(\begin{array}{c}
W_{\mu}^{3} \\
B_{\mu}
\end{array}\right)=\frac{1}{\sqrt{g_{1}^{2}+g_{W}^{2}}}\left(\begin{array}{cc}
g_{W} & g_{1} \\
-g_{1} & g_{W}
\end{array}\right)\left(\begin{array}{cc}
1 & \frac{1}{2} \frac{g_{W}^{2}-g_{1}^{2}}{g_{W}^{2}+g_{1}^{2}} \bar{C}_{H W B} \\
-\frac{1}{2} \frac{g_{W}^{2}-g_{1}^{2}}{g_{W}^{2}+g_{1}^{2}} \bar{C}_{H W B} & 1
\end{array}\right)\left(\begin{array}{c}
Z_{\mu} \\
A_{\mu}
\end{array}\right)
$$

The rightmost rotation is unitary up to $\mathcal{O}\left(\Lambda^{-4}\right)$ corrections, and therefore does not reintroduce kinetic mixing at $d=6$. Equivalently,

$$
\left(\begin{array}{c}
W_{\mu}^{3} \\
B_{\mu}
\end{array}\right)=\left(\begin{array}{cc}
\cos \theta & \sin \theta \\
-\sin \theta & \cos \theta
\end{array}\right)\left(\begin{array}{c}
Z_{\mu} \\
A_{\mu}
\end{array}\right),
$$

with a shifted weak mixing angle $\theta$ defined as

$$
\tan \theta=\frac{g_{1}}{g_{W}}+\frac{1}{2} \bar{C}_{H W B}\left(1-\frac{g_{1}^{2}}{g_{W}^{2}}\right) .
$$

After all the coupling and field redefinitions have been applied, a generic covariant derivative has the form

$$
\begin{aligned}
D_{\mu}= & \partial_{\mu}+i Q \frac{g_{1} g_{W}}{\sqrt{g_{1}^{2}+g_{W}^{2}}} A_{\mu}\left[1-\bar{C}_{H W B} \frac{g_{1} g_{W}}{g_{W}^{2}+g_{1}^{2}}\right] \\
& +i \sqrt{g_{1}^{2}+g_{W}^{2}} Z_{\mu}\left[T_{3}-\frac{g_{1}^{2}}{g_{1}^{2}+g_{W}^{2}} Q+\bar{C}_{H W B} \frac{g_{1} g_{W}}{g_{1}^{2}+g_{W}^{2}}\left(T_{3}-\frac{g_{W}^{2}}{g_{1}^{2}+g_{W}^{2}} Q\right)\right] \\
& +\ldots \\
= & \partial_{\mu}+i Q g_{W} s_{\theta} A_{\mu}\left[1-\frac{1}{2} \frac{c_{\theta}}{s_{\theta}} \bar{C}_{H W B}\right]+i \frac{g_{W}}{c_{\theta}} Z_{\mu}\left(T_{3}-Q s_{\theta}^{2}\right)\left[1+\frac{1}{2} \frac{s_{\theta}}{c_{\theta}} \bar{C}_{H W B}\right] \\
& +\ldots
\end{aligned}
$$

where $T_{3}$ denotes the eigenvalue of the $3^{\text {rd }} \mathrm{SU}(2)_{L}$ generator $\left(T_{3}= \pm 1 / 2\right.$ for left-handed fields and $T_{3}=0$ for right-handed ones) and $Q=T_{3}+\mathbf{y}$ is the electric charge. We have also introduced the shorthand notation $s_{\theta}=\sin \theta, c_{\theta}=\cos \theta$, with $\theta$ defined as in eq. (2.17). The dots stand for potential gluon and $W^{ \pm}$terms, for which there are no residual $\mathcal{L}_{6}$ corrections.

Eq. (2.18) shows that the contributions from $Q_{H W}, Q_{H B}, Q_{H G}$ are fully reabsorbed in the definition of the fields and gauge couplings. As a consequence, these operators have no physical impact in the pure gauge sector, and they only contribute to Higgs-gauge interactions [63]. On the other hand, the operator $Q_{H W B}$ introduces net modifications of all $\gamma$ and $Z$ couplings. In the former case the correction is a universal rescaling of the 
electromagnetic constant, while in the latter case the corrections depend on the field's charges. In particular, in the Higgs case $\left(T_{3}=-1 / 2, Q=0\right)$ this implies a correction $\propto \bar{C}_{H W B}$ to the $Z$ mass term. The physical interpretation of these contributions requires defining a set of input observables and is deferred to section 4 .

In SMEFTsim, the redefinitions described in this subsection are applied simultaneously at the Lagrangian level in the FeynRules model. The coupling constants' rescaling in eq. (2.11) is implemented in the replacement list redefConst. The field redefinitions are operated in the mass and charge eigenstate basis: the replacement list rotateGauge $B$ implements the net mismatch between the series of rotations (2.10), (2.13), (2.15) and the usual SM rotations, i.e.

$$
\begin{aligned}
G_{\mu}^{a} & \rightarrow\left(1+\bar{C}_{H G}\right) G_{\mu}^{a}, \\
\left(\begin{array}{c}
W_{\mu}^{+} \\
W_{\mu}^{-}
\end{array}\right) & \rightarrow\left(1+\bar{C}_{H W}\right)\left(\begin{array}{c}
W_{\mu}^{+} \\
W_{\mu}^{-}
\end{array}\right), \\
\left(\begin{array}{c}
Z_{\mu} \\
A_{\mu}
\end{array}\right) & \rightarrow R_{Z A}\left(\begin{array}{c}
Z_{\mu} \\
A_{\mu}
\end{array}\right),
\end{aligned}
$$

with

$$
R_{Z A}=\left(\begin{array}{cc}
1+c_{\theta}^{2} \bar{C}_{H W}+s_{\theta}^{2} \bar{C}_{H B}+\frac{s_{2 \theta}}{2} \bar{C}_{H W B} & \frac{s_{2 \theta}}{2}\left(\bar{C}_{H W}-\bar{C}_{H B}\right)-\frac{c_{2 \theta}}{2} \bar{C}_{H W B}+\frac{\Delta s_{\theta}^{2}}{s_{2 \theta}} \\
\frac{s_{2 \theta}}{2}\left(\bar{C}_{H W}-\bar{C}_{H B}\right)-\frac{c_{2 \theta}}{2} \bar{C}_{H W B}-\frac{\Delta s_{\theta}^{2}}{s_{2 \theta}} & 1+c_{\theta}^{2} \bar{C}_{H B}+s_{\theta}^{2} \bar{C}_{H W}-\frac{s_{2 \theta}}{2} \bar{C}_{H W B}
\end{array}\right),
$$

where

$$
\Delta s_{\theta}^{2}=\bar{C}_{H W B} g_{1} g_{W} \frac{g_{W}^{2}-g_{1}^{2}}{g_{W}^{2}+g_{1}^{2}}=\frac{s_{4 \theta}}{4} \bar{C}_{H W B}+\mathcal{O}\left(\Lambda^{4}\right)
$$

is the correction to the mixing angle stemming from eq. (2.17).

\section{$3 \quad$ Flavor assumptions}

The SMEFT Lagrangian defined in section 1 is not invariant under flavor rotations of the fermion fields, so the latter should always be defined in order to avoid ambiguities. In SMEFTsim, the fields $q, l, u, d, e$ are defined in the mass basis of the charged leptons and of the up-type quarks, in which the Yukawa matrices in eq. (1.5) take the form

$$
Y_{d} \equiv Y_{d}^{(d)} V^{\dagger}, \quad Y_{u} \equiv Y_{u}^{(d)}, \quad Y_{l} \equiv Y_{l}^{(d)} .
$$

The superscript $(d)$ denotes diagonal matrices and $V$ is the CKM matrix. This basis choice is consistently employed in the definition of both $\mathcal{L}_{\mathrm{SM}}$ and $\mathcal{L}_{6}$, and for all the flavor assumptions implemented in SMEFTsim. The only special case are the top and topU31 models, where quark mixing is entirely neglected by setting $V \equiv \mathbb{1}$. 
Upon EWSB, the Lagrangian can be written in terms of the fermionic mass eigenstates. By definition the relation between the $\mathrm{SU}(2)_{L}$ and mass bases is trivial for all fermion fields, except the left-handed quark doublet: ${ }^{6}$

$$
q_{p}=\left(\begin{array}{c}
u_{L, p} \\
V_{p r} d_{L, r}
\end{array}\right), \quad l_{p}=\left(\begin{array}{c}
\nu_{L, p} \\
e_{L, p}
\end{array}\right), \quad u_{p}=u_{R, p}, \quad d_{p}=d_{R, p}, \quad e_{p}=e_{R, p} .
$$

In unitary gauge, the relevant terms in the SM Lagrangian are therefore

$$
\begin{aligned}
\mathcal{L}_{\text {Fermions }} & =-\frac{g_{W}}{\sqrt{2}} W_{\mu}^{+} \bar{u}_{L} \gamma^{\mu} V d_{L}-\frac{g_{W}}{\sqrt{2}} W_{\mu}^{+} \bar{\nu}_{L} \gamma^{\mu} e_{L}+\ldots \\
\mathcal{L}_{\text {Yukawa }} & =-\frac{v_{T}+h}{\sqrt{2}}\left[\bar{d}_{R} Y_{d}^{(d)} d_{L}+\bar{u}_{R} Y_{u}^{(d)} u_{L}+\bar{e}_{R} Y_{l}^{(d)} e_{L}\right]+\text { h.c. } .
\end{aligned}
$$

The CKM matrix is implemented in the Wolfenstein parameterization [64]:

$$
V=\left(\begin{array}{ccc}
1-\lambda_{C K M}^{2} / 2 & \lambda_{C K M} & A \lambda_{C K M}^{3}(\rho-i \eta) \\
-\lambda_{C K M} & 1-\lambda_{C K M}^{2} / 2 & A \lambda_{C K M}^{2} \\
A \lambda_{C K M}^{3}(1-\rho-i \eta) & -A \lambda_{C K M}^{2} & 1
\end{array}\right) .
$$

The numerical values employed for the parameters are listed in table 16 .

SMEFTsim implements five alternative flavor scenarios: one with fully arbitrary indices, and four based on the implementation of different global symmetries. Three of these scenarios have been present since the first release, and two have been newly introduced in version 3.0. The following sub-sections review in detail the properties of the $\mathcal{L}_{6}$ operators within each setup and provide the corresponding parameter counting. A dictionary between the different flavor assumptions is provided in appendix C.

\section{1 general: general flavor structure}

Without further assumptions on the flavor structure of the SMEFT, $\mathcal{L}_{6}$ contains the operators in table 1 , summed over all possible flavor combinations:

$$
\begin{aligned}
\mathcal{L}_{6}^{(5,6)} & =\frac{1}{\Lambda^{2}} \sum_{\alpha} \sum_{p, r=1}^{3} C_{\alpha, p r} Q_{\alpha, p r}+\text { h.c. }, \quad \mathcal{L}_{6}^{(7)}=\frac{1}{\Lambda^{2}} \sum_{\alpha} \sum_{p, r=1}^{3} C_{\alpha, p r} Q_{\alpha, p r}, \\
\mathcal{L}_{6}^{(8 a, 8 b, 8 c)} & =\frac{1}{\Lambda^{2}} \sum_{\alpha} \sum_{p, r, s, t=1}^{3} C_{\alpha, p r s t} Q_{\alpha, p r s t}, \quad \mathcal{L}_{6}^{(8 d)}=\frac{1}{\Lambda^{2}} \sum_{\alpha} \sum_{p, r, s, t=1}^{3} C_{\alpha, p r s t} Q_{\alpha, p r s t}+\text { h.c. } .
\end{aligned}
$$

Not all flavor combinations included in this way are independent, due to intrinsic symmetry properties of the effective operators. SMEFTsim does not remove redundant terms from the sums in eqs. (3.6), (3.7). Instead, the symmetry relations are enforced in the definition of the tensor Wilson coefficients $C_{\alpha, p r(s t)}$ : only a minimum number of independent parameters is defined for each operator, as reported in appendix D, tables 18, 19, and all the entries of $C_{\alpha, p r(s t)}$ are functions of these parameters, consistent with the relations described below.

\footnotetext{
${ }^{6}$ For economy of notation, we use the same letters $u, d, e$ for the right-handed fields and for the mass eigenstates, both of them carrying flavor indices. To avoid ambiguities, the latter always carry $L, R$ subscripts, while the former don't.
} 
Classes 5 and 6. The operators in $\mathcal{L}_{6}^{(5,6)}$ are not Hermitian. Therefore each Wilson coefficient has 9 independent complex entries. In total, this gives 198 real parameters (counting independently real and imaginary parts).

Class 7. All operators in $\mathcal{L}_{6}^{(7)}$, except $Q_{H u d}$, are Hermitian. In this case, the diagonal entries of the Wilson coefficients are real, and the off-diagonal ones are related by

$$
C_{p r}=C_{r p}^{*}
$$

In total, this class depends on 81 real parameters.

Class 8 a. All operators in $\mathcal{L}_{6}^{(8 a)}$ are Hermitian. Moreover, each of the two currents that compose them is itself Hermitian. Therefore the following relation holds:

$$
C_{p r s t}=C_{r p t s}=C_{r p s t}^{*}=C_{p r t s}^{*}
$$

In the operators $Q_{l l}, Q_{q q}^{(1)}, Q_{q q}^{(3)}$, the two currents contain the same fields, which leads to an additional exchange symmetry

$$
C_{\text {prst }}=C_{\text {stpr }} .
$$

Each of these three operators has then 15 real entries $\left(C_{p p p p}, C_{p p r r}, C_{p r r p}\right)$ and only 9 are independent, and 66 complex entries, 18 independent. Operators $Q_{l q}^{(1)}, Q_{l q}^{(3)}$ have each 9 real entries, all independent, and 72 complex ones, only 36 independent.

In total, this class depends on 297 real parameters.

Class 8 b. All operators in $\mathcal{L}_{6}^{(8 b)}$ are Hermitian and composed of two Hermitian currents, so relation (3.9) holds for all Wilson coefficients in this class. Eq. (3.10) is valid in addition for $C_{u u}, C_{d d}, C_{e e}$.

The operator $Q_{e e}$ is peculiar: because the $e$ field is a singlet under both $\mathrm{SU}(2)_{L}$ and $\mathrm{SU}(3)_{c}$, this term is invariant under Fierz rearranging. This leads to the additional constraint

$$
C_{p r s t}=C_{p t s r}
$$

The coefficient $C_{e e}$ has then 15 real entries, 6 independent, and 66 complex entries, only 15 independent. The counting for the other operators is the same as for the invariants in class $8 \mathrm{a}$, so $\mathcal{L}_{6}^{(8 b)}$ has a total of 450 real parameters.

Class 8 c. All operators in $\mathcal{L}_{6}^{(8 c)}$ are Hermitian and composed of two Hermitian currents, but no other symmetry is present. Therefore only relation (3.9) holds for all Wilson coefficients, leaving a total of 648 parameters.

Class 8 d. Finally, all operators in $\mathcal{L}_{6}^{8 d}$ are non-Hermitian. No symmetry relation is present and this class has 810 independent real parameters. 


\subsection{U35: maximal U(3) ${ }^{5}$ symmetry}

The number of independent parameters is considerably reduced if a flavor symmetry is assumed. The maximal symmetry available for the SM fermion fields is the symmetry of the kinetic terms [65]: $\mathrm{U}(3)^{5}=\mathrm{U}(3)_{q} \times \mathrm{U}(3)_{u} \times \mathrm{U}(3)_{d} \times \mathrm{U}(3)_{l} \times \mathrm{U}(3)_{e}$. Each field is assigned to a 3 representation of the associated group: denoting a generic $\mathrm{U}(3)_{\psi}$ transformation by $\Omega_{\psi}$, the transformation rules are [48]

$$
q \mapsto \Omega_{q} q, \quad u \mapsto \Omega_{u} u, \quad d \mapsto \Omega_{d} d, \quad l \mapsto \Omega_{l} l, \quad e \mapsto \Omega_{e} e
$$

Vector currents $\bar{\psi}_{p} \gamma^{\mu} \psi_{r}$ are trivially made invariant under $\mathrm{U}(3)^{5}$ by imposing a $\delta_{p r}$ contraction, that corresponds to the singlet composition of a $\overline{\mathbf{3}}$ and $\mathbf{3}$ representations. This is immediate to see applying the field transformations and using $\Omega_{\psi} \Omega_{\psi}^{\dagger}=\mathbb{1}=\Omega_{\psi}^{\dagger} \Omega_{\psi}$ :

$$
\bar{\psi} \gamma^{\mu} \psi \mapsto \bar{\psi} \Omega_{\psi}^{\dagger} \gamma^{\mu} \Omega_{\psi} \psi=\bar{\psi} \gamma^{\mu} \psi, \quad \psi=\{q, u, d, l, e\}
$$

Chirality-flipping currents, with either scalar or tensor Lorentz structure, violate the flavor symmetry. To permit the introduction of fermion masses, it is customary to promote the Yukawa couplings to spurions of the flavor symmetry, by assigning them transformation properties

$$
Y_{d} \mapsto \Omega_{d} Y_{d} \Omega_{q}^{\dagger}, \quad Y_{u} \mapsto \Omega_{u} Y_{u} \Omega_{q}^{\dagger}, \quad Y_{l} \mapsto \Omega_{e} Y_{l} \Omega_{l}^{\dagger} .
$$

In this way the structures

$$
\bar{d} Y_{d} q^{i}, \quad \bar{u} Y_{u} q^{i}, \quad \bar{e} Y_{l} l^{i},
$$

are formally invariant. When the $\mathrm{U}(3)^{5}$ symmetry is imposed, the flavor structure of each operator can be factored out of the Wilson coefficient, that becomes a scalar quantity:

$$
\begin{aligned}
\mathcal{L}_{6}^{(5,6)} & =\frac{1}{\Lambda^{2}} \sum_{\alpha} \sum_{p, r=1}^{3} C_{\alpha} X_{\alpha, p r} Q_{\alpha, p r}+\text { h.c. }, & \mathcal{L}_{6}^{(7)}=\frac{1}{\Lambda^{2}} \sum_{\alpha} \sum_{p, r=1}^{3} C_{\alpha} X_{\alpha, p r} Q_{\alpha, p r}, \\
\mathcal{L}_{6}^{(8 a, 8 b, 8 c)} & =\frac{1}{\Lambda^{2}} \sum_{\alpha} \sum_{p, r, s, t=1}^{3} C_{\alpha} X_{\alpha, p r s t} Q_{\alpha, p r s t}, & \mathcal{L}_{6}^{(8 d)}=\frac{1}{\Lambda^{2}} \sum_{\alpha} \sum_{p, r, s, t=1}^{3} C_{\alpha} X_{\alpha, p r s t} Q_{\alpha, p r s t}+\text { h.c. } .
\end{aligned}
$$

In the construction of the $\mathrm{U}(3)^{5}$ symmetric Lagrangian, we do not define a power counting for insertions of the Yukawa couplings. Instead, we simply choose to retain the leading invariant structures for each operator, corresponding to no Yukawa insertions in $\mathcal{L}_{6}^{(7,8 a, 8 b, 8 c)}$, one insertion in $\mathcal{L}_{6}^{(5,6)}$ and two insertions in $C_{H u d}$ and $\mathcal{L}_{6}^{(8 d)}$.

Classes 5 and 6. All the operators in $\mathcal{L}_{6}^{(5,6)}$ require the insertion of a Yukawa coupling:

$$
\begin{gathered}
X_{e H}=X_{e W}=X_{e B}=Y_{l}^{\dagger}=Y_{l}^{(d)}, \\
X_{u H}=X_{u W}=X_{u B}=X_{u G}=Y_{u}^{\dagger}=Y_{u}^{(d)}, \\
X_{d H}=X_{d W}=X_{d B}=X_{d G}=Y_{d}^{\dagger}=V Y_{d}^{(d)},
\end{gathered}
$$


where the last equality in each line holds in the up-quarks mass basis, eq. (3.1). Note that no net mixing among down-type quarks is induced in the mass basis, as the $V$ in the spurion cancels against $V^{\dagger}$ in the $\bar{q}$ field, eq. (3.2). In fact, by construction all the operators in $\mathcal{L}_{6}^{(5,6)}$ have the same flavor structure as the SM Yukawas.

Because the operators are non-Hermitian, the associated $C_{\alpha}$ are complex. These classes therefore introduce 22 independent real parameters.

Class 7. All the currents appearing in the operators of class 7, except $Q_{H u d}$, are invariant under $\mathrm{U}(3)$ with $X_{\alpha}=\mathbb{1}$. This implies that the flavor structure of this class is exactly the same as in the SM kinetic terms. For instance, the charged quark current induced by the operator $Q_{H q}^{(3)}$ is aligned with the SM one (eq. (3.3)), that contains CKM mixing. ${ }^{7}$

In order to make $Q_{H u d}$ invariant, it is necessary to insert the spurion product

$$
X_{H u d}=Y_{u} Y_{d}^{\dagger}=Y_{u}^{(d)} V Y_{d}^{(d)}
$$

The number of independent real parameters is 9 , as 7 out of 8 operators are Hermitian.

Class 8 a. Containing only vector currents, all the operators in $\mathcal{L}_{6}^{(8 a)}$ are $\mathrm{U}(3)^{5}$ invariant with the trivial flavor contraction $X_{\alpha}=\delta_{p r} \delta_{s t}$.

The operators $Q_{l l}, Q_{q q}^{(1)}, Q_{q q}^{(3)}$ additionally admit the "crossed" contraction $X_{\alpha}^{\prime}=\delta_{p t} \delta_{s r}$. This is an independent structure that cannot be arbitrarily rearranged into $X_{\alpha}$ : applying Fierz transformations in this case would introduce additional operators with $\mathrm{SU}(2)_{L}$ triplet and $\mathrm{SU}(3)_{c}$ octet contractions, see section 3.4. Therefore these operators are split into two invariants each, weighted by independent Wilson coefficients:

$$
\Lambda^{2} \mathcal{L}_{6}^{(8 a)}=\left(C_{l l} \delta_{p r} \delta_{s t}+C_{l l}^{\prime} \delta_{p t} \delta_{s r}\right) Q_{l l, p r s t}+\left(C_{q q}^{(1)} \delta_{p r} \delta_{s t}+C_{q q}^{(1) \prime} \delta_{p t} \delta_{s r}\right) Q_{q q, p r s t}^{(1)}+\ldots
$$

where the dots stand for contributions from the other operators in $\mathcal{L}_{6}^{(8 a)}$.

As all operators are Hermitian, $\mathcal{L}_{6}^{(8 a)}$ contains 8 real parameters.

Class 8 b. All operators in $\mathcal{L}_{6}^{(8 b)}$ are invariant with $X_{\alpha}=\delta_{p r} \delta_{s t}$.

The operators $Q_{u u}, Q_{d d}$ additionally admit independent crossed contractions $X_{\alpha}^{\prime}=$ $\delta_{p t} \delta_{s r}$, and are treated analogously to $Q_{l l}, Q_{q q}^{(1),(3)}$. This is not the case for $Q_{e e}$ that, as mentioned above, is invariant under Fierz rearrangements: in this particular case the two flavor contractions are equivalent. In total, there are 9 real parameters in $\mathcal{L}_{6}^{(8 b)}$.

Class 8 c. All the operators in $\mathcal{L}_{6}^{(8 c)}$ admit the invariant contraction is $X_{\alpha}=\delta_{p r} \delta_{s t}$, leading to 8 independent real parameters.

Class 8 d. Finally, operators in $\mathcal{L}_{6}^{(8 d)}$ require one Yukawa coupling insertion for each current. As they are not invariant under Fierz transformations, the operators $Q_{q u q d}^{(1),(8)}$

\footnotetext{
${ }^{7}$ Note that this implies that, even though all Wilson coefficients are real, SM-sourced CP violation, due to the CKM phase in charged left-handed currents, is generally present in $\mathcal{L}_{6}^{(7)}$.
} 
admit two independent contractions, mapped to one another by interchanging the two $\bar{q}$ fields. ${ }^{8}$

$$
\begin{aligned}
& X_{\text {ledq }}=\left(Y_{l}^{\dagger}\right)_{p r}\left(Y_{d}\right)_{s t}=\left(Y_{l}^{(d)}\right)_{p r}\left(Y_{d}^{(d)} V^{\dagger}\right)_{s t}, \\
& X_{q u q d}^{(1)}=X_{q u q d}^{(8)}=\left(Y_{u}^{\dagger}\right)_{p r}\left(Y_{d}^{\dagger}\right)_{s t}=\left(Y_{u}^{(d)}\right)_{p r}\left(V Y_{d}^{(d)}\right)_{s t}, \\
& X_{q u q d}^{(1) \prime}=X_{q u q d}^{(8) \prime}=\left(Y_{u}^{\dagger}\right)_{s r}\left(Y_{d}^{\dagger}\right)_{p t}=\left(Y_{u}^{(d)}\right)_{s r}\left(V Y_{d}^{(d)}\right)_{p t}, \\
& X_{\text {lequ }}^{(1)}=X_{\text {lequ }}^{(3)}=\left(Y_{l}^{\dagger}\right)_{p r}\left(Y_{u}^{\dagger}\right)_{s t}=\left(Y_{l}^{(d)}\right)_{p r}\left(Y_{u}^{(d)}\right)_{s t} .
\end{aligned}
$$

Because the operators are non-Hermitian, there are 14 real parameters in $\mathcal{L}_{6}^{(8 d)}$.

\subsection{MFV: linear minimal flavor violation}

The Minimal Flavor Violation ansatz [65, 67, 68] assumes that the only sources of flavor and $\mathrm{CP}$ violation in $\mathcal{L}_{\mathrm{SMEFT}}$ are those already present in the SM, namely the Yukawa couplings and the CKM phase.

The requirement on $\mathrm{CP}$ violation implies that the Wilson coefficients of $\mathrm{CP}$-odd bosonic operators scale with the Jarlskog invariant $J[69,70]$ :

$$
\begin{aligned}
& \left\{C_{\widetilde{G}}, C_{\widetilde{W}}, C_{H \widetilde{W}}, C_{H \widetilde{B}}, C_{H \widetilde{W} B}, C_{H \widetilde{G}}\right\} \propto J, \\
& J=\operatorname{Im}\left(V_{p r} V_{s t} V_{p t}^{*} V_{s r}^{*}\right) \simeq \eta A^{2} \lambda_{C K M}^{6}\left(1-\frac{\lambda_{C K M}^{2}}{2}\right) \simeq 3 \times 10^{-5} .
\end{aligned}
$$

As the $J$ suppression is stronger, for instance, than a loop factor, these coefficients can be safely neglected within the scope of SMEFTsim. The corresponding operators are therefore not implemented in the MFV version. An analogous argument applies to sources of explicit $\mathrm{CP}$ violation in the fermion sector. In the Warsaw basis, these are the imaginary parts of the Wilson coefficients in $\mathcal{L}_{6}$, that are not defined either in the SMEFTsim MFV models.

The requirement on flavor violation is realized imposing a $\mathrm{U}(3)^{5}$ symmetry on the fermion fields and allowing for arbitrary $\mathrm{U}(3)^{5}$-invariant spurion insertions in the currents, that generate flavor violating effects. Such insertions are organized in an expansion in powers of the Yukawa couplings, that can be either resummed (obtaining a non-linear MFV formulation [71]) or treated as a truncated series. SMEFTsim adopts the latter option and retains contributions up to one power of $Y_{l}$ and up to 3 powers of $Y_{u}, Y_{d}$.

The relevant spurion structures at this order are

$$
\begin{array}{rlrl}
S^{u} & =Y_{u} Y_{u}^{\dagger} & \sim(\mathbf{1}, \mathbf{8}, \mathbf{1}), & S^{u} \mapsto \Omega_{u} S^{u} \Omega_{u}^{\dagger}, \\
S^{d}=Y_{d} Y_{d}^{\dagger} & \sim(\mathbf{1}, \mathbf{1}, \mathbf{8}), & S^{d} \mapsto \Omega_{d} S^{d} \Omega_{d}^{\dagger}, \\
S^{q u}=Y_{u}^{\dagger} Y_{u} & \sim(\mathbf{8}, \mathbf{1}, \mathbf{1}), & S^{q u} \mapsto \Omega_{q} S^{q u} \Omega_{q}^{\dagger}, \\
S^{q d}=Y_{d}^{\dagger} Y_{d} & \sim(\mathbf{8}, \mathbf{1}, \mathbf{1}), & S^{q d} \mapsto \Omega_{q} S^{q d} \Omega_{q}^{\dagger} .
\end{array}
$$

The first column indicates the spurions' representation under the $\mathrm{U}(3)_{q} \times \mathrm{U}(3)_{u} \times \mathrm{U}(3)_{d}$ group, while the second provides the corresponding transformation rules. All of them are

\footnotetext{
${ }^{8}$ The two $X_{\alpha}^{\prime}$ structures (4 real parameters) for $Q_{q u q d}^{(1),(8)}$ were not included in previous versions of SMEFTsim. I thank the authors of ref. [66] for pointing this out.
} 
Hermitian and they satisfy

$$
\begin{array}{ll}
Y_{u}^{\dagger} S^{u}=S^{q u} Y_{u}^{\dagger}, & Y_{u} S^{q u}=S^{u} Y_{u}, \\
Y_{d}^{\dagger} S^{d}=S^{q d} Y_{d}^{\dagger}, & Y_{d} S^{q d}=S^{d} Y_{d} .
\end{array}
$$

In the mass basis of the up quarks (eq. (3.1)) the spurions take the form

$$
\begin{array}{rlrl}
S^{u} & \equiv\left(Y_{u}^{(d)}\right)^{2}, & S^{q u} \equiv\left(Y_{u}^{(d)}\right)^{2}=S^{u}, \\
S^{d} \equiv\left(Y_{d}^{(d)}\right)^{2}, & S^{q d} \equiv V\left(Y_{d}^{(d)}\right)^{2} V^{\dagger} .
\end{array}
$$

Additional relevant structures in this basis are

$$
\begin{aligned}
S^{q u} Y_{u}^{\dagger} & =\left(Y_{u}^{(d)}\right)^{3}, & S^{q u} Y_{d}^{\dagger} & =\left(Y_{u}^{(d)}\right)^{2} V Y_{d}^{(d)}, \\
S^{q d} Y_{u}^{\dagger} & =V\left(Y_{d}^{(d)}\right)^{2} V^{\dagger} Y_{u}^{(d)}, & S^{q d} Y_{d}^{\dagger} & =V\left(Y_{d}^{(d)}\right)^{3} .
\end{aligned}
$$

Classes 5 and 6. With the power counting chosen, $\mathcal{L}_{6}^{(5,6)}$ take the form

$$
\begin{aligned}
\Lambda^{2} \mathcal{L}_{6}^{(5)}=\sum_{p, r=1}^{3} & C_{e H}\left(Y_{l}^{\dagger}\right)_{p r} Q_{e H, p r} \\
& +\left[C_{u H}^{(0)} Y_{u}^{\dagger}+\left(\Delta^{u} C_{u H}\right) S^{q u} Y_{u}^{\dagger}+\left(\Delta^{d} C_{u H}\right) S^{q d} Y_{u}^{\dagger}\right]_{p r} Q_{u H, p r} \\
& +\left[C_{d H}^{(0)} Y_{d}^{\dagger}+\left(\Delta^{u} C_{d H}\right) S^{q u} Y_{d}^{\dagger}+\left(\Delta^{d} C_{d H}\right) S^{q d} Y_{d}^{\dagger}\right]_{p r} Q_{d H, p r} \\
& + \text { h.c. }, \\
\Lambda^{2} \mathcal{L}_{6}^{(6)}=\sum_{p, r=1}^{3} & C_{e W}\left(Y_{l}^{\dagger}\right)_{p r} Q_{e W, p r}+C_{e B}\left(Y_{l}^{\dagger}\right)_{p r} Q_{e B, p r} \\
& +\left[C_{u G}^{(0)} Y_{u}^{\dagger}+\left(\Delta^{u} C_{u G}\right) S^{q u} Y_{u}^{\dagger}+\left(\Delta^{d} C_{u G}\right) S^{q d} Y_{u}^{\dagger}\right]_{p r} Q_{u G, p r} \\
& +\left[C_{u W}^{(0)} Y_{u}^{\dagger}+\left(\Delta^{u} C_{u W}\right) S^{q u} Y_{u}^{\dagger}+\left(\Delta^{d} C_{u W}\right) S^{q d} Y_{u}^{\dagger}\right]_{p r} Q_{u W, p r} \\
& +\left[C_{u B}^{(0)} Y_{u}^{\dagger}+\left(\Delta^{u} C_{u B}\right) S^{q u} Y_{u}^{\dagger}+\left(\Delta^{d} C_{u B}\right) S^{q d} Y_{u}^{\dagger}\right]_{p r} Q_{u B, p r} \\
& +\left[C_{d G}^{(0)} Y_{d}^{\dagger}+\left(\Delta^{u} C_{d G}\right) S^{q u} Y_{d}^{\dagger}+\left(\Delta^{d} C_{d G}\right) S^{q d} Y_{d}^{\dagger}\right]_{p r} Q_{d G, p r} \\
& +\left[C_{d W}^{(0)} Y_{d}^{\dagger}+\left(\Delta^{u} C_{d W}\right) S^{q u} Y_{d}^{\dagger}+\left(\Delta^{d} C_{d W}\right) S^{q d} Y_{d}^{\dagger}\right]_{p r} Q_{d W, p r} \\
& +\left[C_{d B}^{(0)} Y_{d}^{\dagger}+\left(\Delta^{u} C_{d B}\right) S^{q u} Y_{d}^{\dagger}+\left(\Delta^{d} C_{d B}\right) S^{q d} Y_{d}^{\dagger}\right]_{p r} Q_{d B, p r} \\
& + \text { h.c. },
\end{aligned}
$$

where the parameters $C_{\alpha}^{(0)},\left(\Delta^{u} C_{\alpha}\right),\left(\Delta^{d} C_{\alpha}\right)$ are real, scalar quantities. The structures $Y_{u}^{\dagger} S^{u}, Y_{d}^{\dagger} S^{d}$ are also allowed for operators $Q_{u X}, Q_{d X}$ respectively, but they are not independent due to eqs. (3.28), (3.29). These two classes contain a total of 27 real parameters. 
Class 7. For the operators in class 7 we have

$$
\begin{aligned}
\Lambda^{2} \mathcal{L}_{6}^{(7)}=\sum_{p, r=1}^{3} & C_{H l}^{(1)} \delta_{p r} Q_{H l, p r}^{(1)}+C_{H l}^{(3)} \delta_{p r} Q_{H l, p r}^{(3)}+C_{H e} \delta_{p r} Q_{H e, p r} \\
+ & {\left[C_{H q}^{(1)(0)} \mathbb{1}+\left(\Delta^{u} C_{H q}^{(1)}\right) S^{q u}+\left(\Delta^{d} C_{H q}^{(1)}\right) S^{q d}\right]_{p r} Q_{H q, p r}^{(1)} } \\
+ & {\left[C_{H q}^{(3)(0)} \mathbb{1}+\left(\Delta^{u} C_{H q}^{(3)}\right) S^{q u}+\left(\Delta^{d} C_{H q}^{(3)}\right) S^{q d}\right]_{p r} Q_{H q, p r}^{(3)} } \\
+ & {\left[C_{H u}^{(0)} \mathbb{1}+\left(\Delta C_{H u}\right) S^{u}\right]_{p r} Q_{H u, p r} } \\
+ & {\left[C_{H d}^{(0)} \mathbb{1}+\left(\Delta C_{H d}\right) S^{d}\right]_{p r} Q_{H d, p r} } \\
+ & {\left[\left[C_{H u d}^{0} Y_{u} Y_{d}^{\dagger}\right]_{p r} Q_{H u d, p r}+\text { h.c. }\right] . }
\end{aligned}
$$

The total number of independent parameters in this class is 14 .

Class 8 a. The operators of class 8 a are composed of the same currents as those of class 7 . MFV corrections have therefore an analogous structure. With the power counting chosen, the independent contractions are

$$
\begin{aligned}
\Lambda^{2} \mathcal{L}_{6}^{(8 a)}=\sum_{p, r, s, t=1}^{3} & {\left[C_{l l} \delta_{p r} \delta_{s t}+C_{l l}^{\prime} \delta_{p t} \delta_{s r}\right] Q_{l l, p r s t} } \\
+ & {\left[C_{q q}^{(1)(0)} \delta_{p r} \delta_{s t}+\left(\Delta^{u} C_{q q}^{(1)}\right) S_{p r}^{q u} \delta_{s t}+\left(\Delta^{d} C_{q q}^{(1)}\right) S_{p r}^{q d} \delta_{s t}+\right.} \\
& \left.C_{q q}^{(1) \prime(0)} \delta_{p t} \delta_{s r}+\left(\Delta^{u} C_{q q}^{(1) \prime}\right) S_{p t}^{q u} \delta_{s r}+\left(\Delta^{d} C_{q q}^{(1) \prime}\right) S_{p t}^{q d} \delta_{s r}\right] Q_{q q, p r s t}^{(1)} \\
+ & {\left[C_{q q}^{(3)(0)} \delta_{p r} \delta_{s t}+\left(\Delta^{u} C_{q q}^{(3)}\right) S_{p r}^{q u} \delta_{s t}+\left(\Delta^{d} C_{q q}^{(3)}\right) S_{p r}^{q d} \delta_{s t}+\right.} \\
& \left.C_{q q}^{(3) \prime(0)} \delta_{p t} \delta_{s r}+\left(\Delta^{u} C_{q q}^{(3) \prime}\right) S_{p t}^{q u} \delta_{s r}+\left(\Delta^{d} C_{q q}^{(3) \prime}\right) S_{p t}^{q d} \delta_{s r}\right] Q_{q q, p r s t}^{(3)} \\
+ & {\left[C_{l q}^{(1)(0)} \delta_{p r} \delta_{s t}+\left(\Delta^{u} C_{l q}^{(1)}\right) \delta_{p r} S_{s t}^{q u}+\left(\Delta^{d} C_{l q}^{(1)}\right) \delta_{p r} S_{s t}^{q d}\right] Q_{l q, p r s t}^{(1)} } \\
+ & {\left[C_{l q}^{(3)(0)} \delta_{p r} \delta_{s t}+\left(\Delta^{u} C_{l q}^{(3)}\right) \delta_{p r} S_{s t}^{q u}+\left(\Delta^{d} C_{l q}^{(3)}\right) \delta_{p r} S_{s t}^{q d}\right] Q_{l q, p r s t}^{(3)} . }
\end{aligned}
$$

In the case of operators $Q_{l l}, Q_{q q}^{(1),(3)}$ two possible flavor contractions are allowed, as discussed in section 3.2. In $Q_{q q}^{(1,3)}$ spurion insertions in the $(s t),(s r)$ currents are redundant by symmetry. Class $8 \mathrm{c}$ contains 20 independent parameters. 
Class 8 b. The MFV Lagrangian in class $8 \mathrm{~b}$ has the form

$$
\begin{aligned}
\Lambda^{2} \mathcal{L}_{6}^{(8 b)}=\sum_{p, r, s, t=}^{3} & C_{e e} \delta_{p r} \delta_{s t} Q_{e e, p r s t} \\
+ & {\left[C_{u u}^{(0)} \delta_{p r} \delta_{s t}+\left(\Delta C_{u u}\right) S_{p r}^{u} \delta_{s t}+C_{u u}^{(0)} \delta_{p t} \delta_{s r}+\left(\Delta C_{u u}^{\prime}\right) S_{p t}^{u} \delta_{s r}\right] Q_{u u, p r s t} } \\
+ & {\left[C_{d d}^{(0)} \delta_{p r} \delta_{s t}+\left(\Delta C_{d d}\right) S_{p r}^{d} \delta_{s t}+C_{d d}^{(0)} \delta_{p t} \delta_{s r}+\left(\Delta C_{d d}^{\prime}\right) S_{p t}^{d} \delta_{s r}\right] Q_{d d, p r s t} } \\
& +\left[C_{e u}^{(0)} \delta_{s t}+\left(\Delta C_{e u}\right) S_{s t}^{u}\right] \delta_{p r} C_{e u, p r s t} \\
& +\left[C_{e d}^{(0)} \delta_{s t}+\left(\Delta C_{e d}\right) S_{s t}^{d}\right] \delta_{p r} C_{e d, p r s t} \\
& +\left[C_{u d}^{(1)(0)} \delta_{p r} \delta_{s t}+\left(\Delta^{u} C_{u d}^{(1)}\right) S_{p r}^{u} \delta_{s t}+\left(\Delta^{d} C_{u d}^{(1)}\right) \delta_{p r} S_{s t}^{d}\right] C_{u d, p r s t}^{(1)} \\
& +\left[C_{u d}^{(8)(0)} \delta_{p r} \delta_{s t}+\left(\Delta^{u} C_{u d}^{(8)}\right) S_{p r}^{u} \delta_{s t}+\left(\Delta^{d} C_{u d}^{(8)}\right) \delta_{p r} S_{s t}^{d}\right] C_{u d, p r s t}^{(8)} .
\end{aligned}
$$

As in the $\mathrm{U}(3)^{5}$ symmetric case, $Q_{u u}, Q_{d d}$ admit two independent flavor contractions, and spurion insertions in only one of their currents is required, by symmetry. Class $8 \mathrm{~b}$ therefore contains a total of 19 independent parameters.

Class 8 c. For class $8 \mathrm{c}$ we have a total of 28 independent parameters:

$$
\begin{aligned}
\Lambda^{2} \mathcal{L}_{6}^{(8 c)}=\sum_{p, r, s, t=1}^{3} & C_{l e} \delta_{p r} \delta_{s t} Q_{l e, p r s t} \\
+ & {\left[C_{l u}^{(0)} \delta_{s t}+\left(\Delta C_{l u}\right) S_{s t}^{u}\right] \delta_{p r} Q_{l u, p r s t} } \\
+ & {\left[C_{l d}^{(0)} \delta_{s t}+\left(\Delta C_{l d}\right) S_{s t}^{d}\right] \delta_{p r} Q_{l d, p r s t} } \\
+ & {\left[C_{q e}^{(0)} \delta_{p r}+\left(\Delta^{u} C_{q e}\right) S_{p r}^{q u}+\left(\Delta^{d} C_{q e}\right) S_{p r}^{q d}\right] \delta_{s t} Q_{q e, p r s t} } \\
+ & {\left[C_{q u}^{(1)(0)} \delta_{p r} \delta_{s t}+\left(\Delta_{1}^{u} C_{q u}^{(1)}\right) S_{p r}^{q u} \delta_{s t}+\left(\Delta_{1}^{d} C_{q u}^{(1)}\right) S_{p r}^{q d} \delta_{s t}+\left(\Delta_{2} C_{q u}^{(1)}\right) \delta_{p r} S_{s t}^{u}+\right.} \\
& \left.C_{q u}^{(1) \prime(0)}\left(Y_{u}^{\dagger}\right)_{p t}\left(Y_{u}\right)_{s r}\right] Q_{q u, p r s t}^{(1)} \\
+ & {\left[C_{q u}^{(8)(0)} \delta_{p r} \delta_{s t}+\left(\Delta_{1}^{u} C_{q u}^{(8)}\right) S_{p r}^{q u} \delta_{s t}+\left(\Delta_{1}^{d} C_{q u}^{(8)}\right) S_{p r}^{q d} \delta_{s t}+\left(\Delta_{2} C_{q u}^{(8)}\right) \delta_{p r} S_{s t}^{u}+\right.} \\
& \left.C_{q u}^{(8) \prime(0)}\left(Y_{u}^{\dagger}\right)_{p t}\left(Y_{u}\right)_{s r}\right] Q_{q u, p r s t}^{(8)} \\
+ & {\left[C_{q d}^{(1)(0)} \delta_{p r} \delta_{s t}+\left(\Delta_{1}^{u} C_{q d}^{(1)}\right) S_{p r}^{q u} \delta_{s t}+\left(\Delta_{1}^{d} C_{q d}^{(1)}\right) S_{p r}^{q d} \delta_{s t}+\left(\Delta_{2} C_{q d}^{(1)}\right) \delta_{p r} S_{s t}^{d}+\right.} \\
& \left.C_{q d}^{(1) \prime(0)}\left(Y_{d}^{\dagger}\right)_{p t}\left(Y_{d}\right)_{s r}\right] Q_{q d, p r s t}^{(1)} \\
+ & {\left[C_{q d}^{(8)(0)} \delta_{p r} \delta_{s t}+\left(\Delta_{1}^{u} C_{q d}^{(8)}\right) S_{p r}^{q u} \delta_{s t}+\left(\Delta_{1}^{d} C_{q d}^{(8)}\right) S_{p r}^{q d} \delta_{s t}+\left(\Delta_{2} C_{q d}^{(8)}\right) \delta_{p r} S_{s t}^{d}+\right.} \\
& \left.C_{q d}^{(8) \prime(0)}\left(Y_{d}^{\dagger}\right)_{p t}\left(Y_{d}\right)_{s r}\right] Q_{q d, p r s t}^{(8)} .
\end{aligned}
$$

Note that the operators $O_{q u}^{(1),(8)}, Q_{q d}^{(1)(8)}$ admit a contraction $[p t][s r]$ with a Yukawa insertion in each current. 
Class 8 d. Finally, the operators of class $8 d$ have the structure

$$
\begin{aligned}
\Lambda^{2} \mathcal{L}_{6}^{(8 d)}=\sum_{p, r, s, t=1}^{3} & {\left[C_{l e d q}^{(0)} Y_{d, s t}+\left(\Delta^{u} C_{l e d q}\right)\left(Y_{d} S^{q u}\right)_{s t}+\left(\Delta^{d} C_{l e d q}\right)\left(Y_{d} S^{q d}\right)_{s t}\right]\left(Y_{l}^{\dagger}\right)_{p r} Q_{\text {ledq }, p r s t} } \\
& +\left[C_{q u q d}^{(1)} Y_{u, p r}^{\dagger} Y_{d, s t}^{\dagger}+C_{q u q d}^{(1) \prime} Y_{u, s r}^{\dagger} Y_{d, p t}^{\dagger}\right] Q_{q u q d, p r s t}^{(1)} \\
& +\left[C_{q u q d}^{(8)} Y_{u, p r}^{\dagger} Y_{d, s t}^{\dagger}+C_{q u q d}^{(8) \prime} Y_{u, s r}^{\dagger} Y_{d, p t}^{\dagger}\right] Q_{q u q d, p r s t}^{(8)} \\
& +\left[C_{\text {lequ }}^{(1)(0)} Y_{u, s t}^{\dagger}+\left(\Delta^{u} C_{l e q u}^{(1)}\right)\left(S^{q u} Y_{u}^{\dagger}\right)_{s t}+\left(\Delta^{d} C_{\text {lequ }}^{(1)}\right)\left(S^{q d} Y_{u}^{\dagger}\right)_{s t}\right]\left(Y_{l}^{\dagger}\right)_{p r} Q_{\text {lequ,prst }}^{(1)} \\
& +\left[C_{\text {lequ }}^{(3)(0)} Y_{u, s t}^{\dagger}+\left(\Delta^{u} C_{\text {lequ }}^{(3)}\right)\left(S^{q u} Y_{u}^{\dagger}\right)_{s t}+\left(\Delta^{d} C_{\text {lequ }}^{(3)}\right)\left(S^{q d} Y_{u}^{\dagger}\right)_{s t}\right]\left(Y_{l}^{\dagger}\right)_{p r} Q_{\text {lequ,prst }}^{(3)} \\
& + \text { h.c. },
\end{aligned}
$$

The total number of parameters is 13 .

\section{4 top, topU31: $\mathrm{U}(2)^{3}$ symmetry in the quark sector}

Two new sets of models have been introduced in version 3.0, that implement a flavor structure consistent with the recommendations of ref. [36] for the SMEFT interpretation of top quark measurements. The formalism builds upon [72-74] and is defined by the following assumptions:

- quarks of the first two generations and quarks of the 3rd are described by independent fields. We denote them respectively by $\left(q_{p}, u_{p}, d_{p}\right)$ with $p=\{1,2\}$ and by $(Q, t, b)$.

- a symmetry $\mathrm{U}(2)^{3}=\mathrm{U}(2)_{q} \times \mathrm{U}(2)_{u} \times \mathrm{U}(2)_{d}$ is imposed on the Lagrangian, under which only the light quarks transform:

$$
q \mapsto \Omega_{q} q, \quad u \mapsto \Omega_{u} u, \quad d \mapsto \Omega_{d} d, \quad Q \mapsto Q, \quad t \mapsto t, \quad b \mapsto b
$$

- mixing effects in the quark sector are neglected and $V_{C K M} \equiv \mathbb{1}$ is assumed.

This choice greatly simplifies the structure of the Lagrangian, as mixing between the light and heavy quarks can only be introduced through extra $\mathrm{U}(2)$ spurions [66, 72].

With this notation, the SM Lagrangian is

$$
\begin{aligned}
& \mathcal{L}_{\text {fermions }}=i \bar{q} \not D q+i \bar{u} \not D u+i \bar{d} \not D d+i \bar{Q} \not D Q+i \bar{t} \not D t+i \bar{b} \not D b+\text { leptons }, \\
& \mathcal{L}_{\text {Yukawa }}=-\bar{d} Y_{d} H^{\dagger} q-\bar{u} Y_{u} \tilde{H}^{\dagger} q-y_{b} \bar{b} H^{\dagger} Q-y_{t} \bar{t} \tilde{H}^{\dagger} Q+\text { leptons },
\end{aligned}
$$

with the Yukawas of the light quarks $Y_{u} \equiv \operatorname{diag}\left(y_{u}, y_{c}\right), Y_{d} \equiv \operatorname{diag}\left(y_{d}, y_{s}\right)$ promoted to spurions of $\mathrm{U}(2)$

$$
Y_{u} \mapsto \Omega_{u} Y_{u} \Omega_{q}^{\dagger}, \quad Y_{d} \mapsto \Omega_{d} Y_{d} \Omega_{q}^{\dagger},
$$

while $y_{t}, y_{b}$ do not transform under any symmetry. As a consequence, only $(\bar{L} R),(\bar{R} L)$ currents with light quarks need to be weighted by Yukawa insertions.

It is convenient to construct a $\mathrm{U}(2)^{3}$ invariant basis mapping the fermionic operators of table 1 to the notation with 6 quark fields. We choose the set given in table 2, where, 
analogously to the $\mathrm{U}(3)^{5}$ case, we retain the least Yukawa-suppressed $\mathrm{U}(2)^{3}$-invariant contractions for each operator in the Warsaw basis.

In the lepton sector we consider two alternative ansätze:

(a) a $\mathrm{U}(1)_{l+e}^{3}=\mathrm{U}(1)_{e} \times \mathrm{U}(1)_{\mu} \times \mathrm{U}(1)_{\tau}$ symmetry under which the fields transform as

$$
\begin{array}{rlrl}
l_{1} & \mapsto e^{i \alpha_{e}} l_{1}, & l_{2} \mapsto e^{i \alpha_{\mu}} l_{2}, & l_{3} \mapsto e^{i \alpha_{\tau}} l_{3}, \\
e_{1} \mapsto e^{i \alpha_{e}} e_{1}, & e_{2} \mapsto e^{i \alpha_{\mu}} e_{2}, & e_{3} \mapsto e^{i \alpha_{\tau}} e_{3} .
\end{array}
$$

This matches the "baseline" scenario in ref. [36] and corresponds to simple flavordiagonality. It is implemented in the top models.

(b) a $\mathrm{U}(3)^{2}=\mathrm{U}(3)_{l} \times \mathrm{U}(3)_{e}$ symmetry under which

$$
l \mapsto \Omega_{l} l, \quad e \mapsto \Omega_{e} e, \quad Y_{l} \mapsto \Omega_{e} Y_{l} \Omega_{l}^{\dagger} .
$$

In the lepton sector, this setup matches exactly the structure of the U35 and MFV models. It is more restrictive compared to $\mathrm{U}(1)_{l+e}^{3}$ and contains fewer free parameters.

It is implemented in the topU31 models.

In the $\mathrm{U}(1)_{l+e}^{3}$ symmetric case, no transformation rule needs to be assigned to $Y_{l}$, as left- and right-handed leptons transform under the same symmetry. This implies that $(\bar{L} R),(\bar{R} L)$ lepton currents are weighted by $Y_{l}$ in the topU31 models but not in the top ones.

Classes 5 and 6. The basis of quark operators for $\mathcal{L}_{6}^{(5)}$ and $\mathcal{L}_{6}^{(6)}$ in table 2 is easily constructed splitting the quark currents for the first 2 and the 3rd generations. Insertions of $Y_{u}^{\dagger}, Y_{d}^{\dagger}$ in light quark currents, that are required for $\mathrm{U}(2)^{3}$ invariance, are embedded in the operator definitions. $\mathcal{L}_{6}^{(5,6)}$ contain in total 32 real parameters (16 complex) coming from quark invariants.

When $\mathrm{U}(1)_{l+e}^{3}$ is imposed (top models) on the lepton fields, $Q_{e H, p r}, Q_{e W, p r}, Q_{e B, p r}$ admit 3 independent contractions each, one per generation. When the more restrictive $\mathrm{U}(3)^{2}$ is imposed (topU31 models), each operator is associated to only one complex Wilson coefficient.

The total number of real independent parameters in $\mathcal{L}_{6}^{(5,6)}$ is therefore 50 in the top case and 38 in the topU31 case. The Lagrangian is

$$
\begin{aligned}
& \Lambda^{2} \mathcal{L}_{6}^{(5)}=C_{u H} Q_{u H}+C_{t H} Q_{t H}+C_{d H} Q_{d H}+C_{b H} Q_{b H} \\
& + \begin{cases}\sum_{p=1}^{3}\left(C_{e H}\right)_{p p} Q_{e H, p p} & \mathrm{U}(1)_{l+e}^{3}[\mathrm{top}] \\
\sum_{p, r=1}^{3} C_{e H}\left(Y_{l}^{\dagger}\right)_{p r} Q_{e H, p r} & \mathrm{U}(3)^{2} \quad[\text { topU3l] }\end{cases} \\
& + \text { h.c. } \\
& \Lambda^{2} \mathcal{L}_{6}^{(6)}=C_{u W} Q_{u W}+C_{u B} Q_{u B}+C_{u G} Q_{u G}+C_{t W} Q_{t W}+C_{t B} Q_{t B}+C_{t G} Q_{t G} \\
& +C_{d W} Q_{d W}+C_{d B} Q_{d B}+C_{d G} Q_{d G}+C_{b W} Q_{b W}+C_{b B} Q_{b B}+C_{b G} Q_{b G}
\end{aligned}
$$

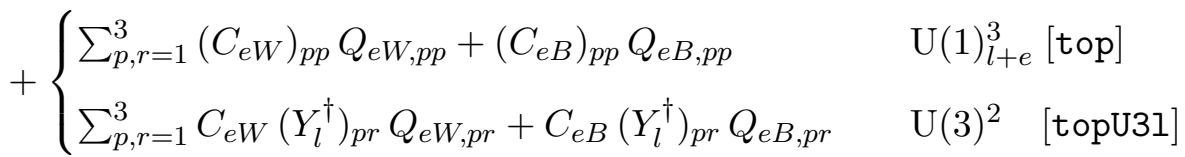

$$
\begin{aligned}
& + \text { h.c. . }
\end{aligned}
$$




\begin{tabular}{|c|c|c|c|c|c|c|c|}
\hline \multicolumn{8}{|c|}{$\mathcal{L}_{6}^{(5)}-\psi^{2} H^{3}$} \\
\hline$Q_{u H}$ & $\left(H^{\dagger} H\right)\left(\bar{q} Y_{u}^{\dagger} u \tilde{H}\right)$ & $Q_{d H}$ & $\left(H^{\dagger} H\right)\left(\bar{q} Y_{d}^{\dagger} d H\right)$ & $Q_{e H}$ & $\left(H^{\dagger} H\right)\left(\bar{l}_{p} e_{r} H\right)$ & & \\
\hline$Q_{t H}$ & $\left(H^{\dagger} H\right)(\bar{Q} \tilde{H} t)$ & $Q_{b H}$ & $\left(H^{\dagger} H\right)(\bar{Q} H b)$ & & & & \\
\hline \multicolumn{8}{|c|}{$\mathcal{L}_{6}^{(6)}-\psi^{2} X H$} \\
\hline$Q_{e W}$ & $\left(\bar{l}_{p} \sigma^{\mu \nu} e_{r}\right) \sigma^{i} H W_{\mu \nu}^{i}$ & $Q_{u W}$ & $\left(\bar{q} Y_{u}^{\dagger} \sigma^{\mu \nu} u\right) \sigma^{i} \tilde{H} W_{\mu \nu}^{i}$ & $Q_{u B}$ & $\left(\bar{q} Y_{u}^{\dagger} \sigma^{\mu \nu} u\right) \tilde{H} B_{\mu \nu}$ & $Q_{u G}$ & $\left(\bar{q} Y_{u}^{\dagger} \sigma^{\mu \nu} T^{a} u\right) \tilde{H} G_{\mu \nu}^{a}$ \\
\hline$Q_{e B}$ & $\left(\bar{l}_{p} \sigma^{\mu \nu} e_{r}\right) H B_{\mu \nu}$ & $Q_{t W}$ & $\left(\bar{Q} \sigma^{\mu \nu} t\right) \sigma^{i} \tilde{H} W_{\mu \nu}^{i}$ & $Q_{t B}$ & $\left(\bar{Q} \sigma^{\mu \nu} t\right) \tilde{H} B_{\mu \nu}$ & $Q_{t G}$ & $\left(\bar{Q} \sigma^{\mu \nu} T^{a} t\right) \tilde{H} G_{\mu \nu}^{a}$ \\
\hline$Q_{d W}$ & $\left(\bar{q} Y_{d}^{\dagger} \sigma^{\mu \nu} d\right) \sigma^{i} H W_{\mu \nu}^{i}$ & $Q_{d B}$ & $\left(\bar{q} Y_{d}^{\dagger} \sigma^{\mu \nu} d\right) H B_{\mu \nu}$ & $Q_{d G}$ & $\left(\bar{q} Y_{d}^{\dagger} \sigma^{\mu \nu} T^{a} d\right) H G_{\mu \nu}^{a}$ & & \\
\hline$Q_{b W}$ & $\left(\bar{Q} \sigma^{\mu \nu} b\right) \sigma^{i} H W_{\mu \nu}^{i}$ & $Q_{b B}$ & $\left(\bar{Q} \sigma^{\mu \nu} b\right) H B_{\mu \nu}$ & $Q_{b G}$ & $\left(\bar{Q} \sigma^{\mu \nu} T^{a} b\right) H G_{\mu \nu}^{a}$ & & \\
\hline \multicolumn{8}{|c|}{$\mathcal{L}_{6}^{(7)}-\psi^{2} H^{2} D$} \\
\hline$Q_{H l}^{(1)}$ & $\left(H^{\dagger} i \overleftrightarrow{D}_{\mu} H\right)\left(\bar{l}_{p} \gamma^{\mu} l_{r}\right)$ & $Q_{H l}^{(3)}$ & $\left(H^{\dagger} i \overleftrightarrow{D}_{\mu}^{i} H\right)\left(\bar{l}_{p} \sigma^{i} \gamma^{\mu} l_{r}\right)$ & $Q_{H e}$ & $\left(H^{\dagger} i \overleftrightarrow{D}_{\mu} H\right)\left(\bar{e}_{p} \gamma^{\mu} e_{r}\right)$ & & \\
\hline$Q_{H q}^{(1)}$ & $\left(H^{\dagger} i \overleftrightarrow{D}_{\mu} H\right)\left(\bar{q} \gamma^{\mu} q\right)$ & $Q_{H q}^{(3)}$ & $\left(H^{\dagger} i \overleftrightarrow{D}_{\mu}^{i} H\right)\left(\bar{q} \sigma^{i} \gamma^{\mu} q\right)$ & $Q_{H u}$ & $\left(H^{\dagger} i \overleftrightarrow{D}_{\mu} H\right)\left(\bar{u} \gamma^{\mu} u\right)$ & $Q_{H d}$ & $\left(H^{\dagger} i \overleftrightarrow{D}_{\mu} H\right)\left(\bar{d} \gamma^{\mu} d\right)$ \\
\hline$Q_{H Q}^{(1)}$ & $\left(H^{\dagger} i \overleftrightarrow{D}_{\mu} H\right)\left(\bar{Q} \gamma^{\mu} Q\right)$ & $Q_{H Q}^{(3)}$ & $\left(H^{\dagger} i \overleftrightarrow{D}_{\mu}^{i} H\right)\left(\bar{Q} \sigma^{i} \gamma^{\mu} Q\right)$ & $Q_{H t}$ & $\left(H^{\dagger} i \overleftrightarrow{D}_{\mu} H\right)\left(\bar{t}^{\mu} t\right)$ & $Q_{H b}$ & $\left(H^{\dagger} i \overleftrightarrow{D}_{\mu} H\right)\left(\bar{b} \gamma^{\mu} b\right)$ \\
\hline$Q_{H u d}$ & $i\left(\tilde{H}^{\dagger} D_{\mu} H\right)\left(\bar{u} Y_{u} Y_{d}^{\dagger} \gamma^{\mu} d\right)$ & $Q_{H t b}$ & $i\left(\tilde{H}^{\dagger} D_{\mu} H\right)\left(\bar{t} \gamma^{\mu} b\right)$ & & & & \\
\hline \multicolumn{8}{|c|}{$\mathcal{L}_{6}^{(8 a)}-(\bar{L} L)(\bar{L} L)$} \\
\hline$Q_{l q}^{(1)}$ & $\left(\bar{l}_{p} \gamma_{\mu} l_{r}\right)\left(\bar{q} \gamma^{\mu} q\right)$ & $Q_{l q}^{(3)}$ & $\left(\bar{l}_{p} \sigma^{i} \gamma_{\mu} l_{r}\right)\left(\bar{q} \sigma^{i} \gamma^{\mu} q\right)$ & $Q_{l l}$ & $\left(\bar{l}_{p} \gamma_{\mu} l_{r}\right)\left(\bar{l}_{s} \gamma^{\mu} l_{t}\right)$ & & \\
\hline$Q_{l Q}^{(1)}$ & $\left(\bar{l}_{p} \gamma_{\mu} l_{r}\right)\left(\bar{Q} \gamma^{\mu} Q\right)$ & $Q_{l Q}^{(3)}$ & $\left(\bar{l}_{p} \sigma^{i} \gamma_{\mu} l_{r}\right)\left(\bar{Q} \sigma^{i} \gamma^{\mu} Q\right)$ & $Q_{Q Q}^{(1)}$ & $\left(\bar{Q} \gamma_{\mu} Q\right)\left(\bar{Q} \gamma^{\mu} Q\right)$ & $Q_{Q Q}^{(8)}$ & $\left(\bar{Q} T^{a} \gamma_{\mu} Q\right)\left(\bar{Q} T^{a} \gamma^{\mu} Q\right)$ \\
\hline$Q_{q q}^{(1,1)}$ & $\left(\bar{q} \gamma_{\mu} q\right)\left(\bar{q} \gamma^{\mu} q\right)$ & $Q_{q q}^{(1,8)}$ & $\left(\bar{q} T^{a} \gamma_{\mu} q\right)\left(\bar{q} T^{a} \gamma^{\mu} q\right)$ & $Q_{q q}^{(3,1)}$ & $\left(\bar{q} \sigma^{i} \gamma_{\mu} q\right)\left(\bar{q} \sigma^{i} \gamma^{\mu} q\right)$ & $Q_{q q}^{(3,8)}$ & $\left(\bar{q} \sigma^{i} T^{a} \gamma_{\mu} q\right)\left(\bar{q} \sigma^{i} T^{a} \gamma^{\mu} q\right)$ \\
\hline$Q_{Q q}^{(1,1)}$ & $\left(\bar{Q} \gamma_{\mu} Q\right)\left(\bar{q} \gamma^{\mu} q\right)$ & $Q_{Q q}^{(1,8)}$ & $\left(\bar{Q} T^{a} \gamma_{\mu} Q\right)\left(\bar{q} T^{a} \gamma^{\mu} q\right)$ & $Q_{Q q}^{(3,1)}$ & $\left(\bar{Q} \sigma^{i} \gamma_{\mu} Q\right)\left(\bar{q} \sigma^{i} \gamma^{\mu} q\right)$ & $Q_{Q q}^{(3,8)}$ & $\left(\bar{Q} \sigma^{i} T^{a} \gamma_{\mu} Q\right)\left(\bar{q} \sigma^{i} T^{a} \gamma^{\mu} q\right)$ \\
\hline \multicolumn{8}{|c|}{$\mathcal{L}_{6}^{(8 b)}-(\bar{R} R)(\bar{R} R)$} \\
\hline$Q_{e u}$ & $\left(\bar{e}_{p} \gamma_{\mu} e_{r}\right)\left(\bar{u} \gamma^{\mu} u\right)$ & $Q_{e d}$ & $\left(\bar{e}_{p} \gamma_{\mu} e_{r}\right)\left(\bar{d} \gamma^{\mu} d\right)$ & $Q_{e e}$ & $\left(\bar{e}_{p} \gamma_{\mu} e_{r}\right)\left(\bar{e}_{s} \gamma^{\mu} e_{t}\right)$ & & \\
\hline$Q_{e t}$ & $\left(\bar{e}_{p} \gamma_{\mu} e_{r}\right)\left(\bar{t} \gamma^{\mu} t\right)$ & $Q_{e b}$ & $\left(\bar{e}_{p} \gamma_{\mu} e_{r}\right)\left(\bar{b} \gamma^{\mu} b\right)$ & $Q_{t t}$ & $\left(\bar{t} \gamma_{\mu} t\right)\left(\bar{t} \gamma^{\mu} t\right)$ & $Q_{b b}$ & $\left(\bar{b} \gamma_{\mu} b\right)\left(\bar{b} \gamma^{\mu} b\right)$ \\
\hline$Q_{u u}^{(1)}$ & $\left(\bar{u} \gamma_{\mu} u\right)\left(\bar{u} \gamma^{\mu} u\right)$ & $Q_{u u}^{(8)}$ & $\left(\bar{u} T^{a} \gamma_{\mu} u\right)\left(\bar{u} T^{a} \gamma^{\mu} u\right)$ & $Q_{t u}^{(1)}$ & $\left(\bar{t} \gamma_{\mu} t\right)\left(\bar{u} \gamma^{\mu} u\right)$ & $Q_{t u}^{(8)}$ & $\left(\bar{t} T^{a} \gamma_{\mu} t\right)\left(\bar{u} T^{a} \gamma^{\mu} u\right)$ \\
\hline$Q_{d d}^{(1)}$ & $\left(\bar{d} \gamma_{\mu} d\right)\left(\bar{d} \gamma^{\mu} d\right)$ & $Q_{d d}^{(8)}$ & $\left(\bar{d} T^{a} \gamma_{\mu} d\right)\left(\bar{d} T^{a} \gamma^{\mu} d\right)$ & $Q_{b d}^{(1)}$ & $\left(\bar{b} \gamma_{\mu} b\right)\left(\bar{d} \gamma^{\mu} d\right)$ & $Q_{b d}^{(8)}$ & $\left(\bar{b} T^{a} \gamma_{\mu} b\right)\left(\bar{d} T^{a} \gamma^{\mu} d\right)$ \\
\hline$Q_{u d}^{(1)}$ & $\left(\bar{u} \gamma_{\mu} u\right)\left(\bar{d} \gamma^{\mu} d\right)$ & $Q_{u d}^{(8)}$ & $\left(\bar{u} T^{a} \gamma_{\mu} u\right)\left(\bar{d} T^{a} \gamma^{\mu} d\right)$ & $Q_{t d}^{(1)}$ & $\left(\bar{\gamma} \gamma_{\mu} t\right)\left(\bar{d} \gamma^{\mu} d\right)$ & $Q_{t d}^{(8)}$ & $\left(\bar{t} T^{a} \gamma_{\mu} t\right)\left(\bar{d} T^{a} \gamma^{\mu} d\right)$ \\
\hline$Q_{u b}^{(1)}$ & $\left(\bar{u} \gamma_{\mu} u\right)\left(\bar{b} \gamma^{\mu} b\right)$ & $Q_{u b}^{(8)}$ & $\left(\bar{u} T^{a} \gamma_{\mu} u\right)\left(\bar{b} T^{a} \gamma^{\mu} b\right)$ & $Q_{t b}^{(1)}$ & $\left(\bar{t} \gamma_{\mu} t\right)\left(\bar{b} \gamma^{\mu} b\right)$ & $Q_{t b}^{(8)}$ & $\left(\bar{t} T^{a} \gamma_{\mu} t\right)\left(\bar{b} T^{a} \gamma^{\mu} b\right)$ \\
\hline$Q_{u t b d}^{(1)}$ & $\left(Y_{u} Y_{d}^{\dagger}\right)_{p r}\left(\bar{u}_{p} \gamma_{\mu} t\right)\left(\bar{b} \gamma^{\mu} d_{r}\right)$ & $Q_{u t b d}^{(8)}$ & $\left(Y_{u} Y_{d}^{\dagger}\right)_{p r}\left(\bar{u}_{p} T^{a} \gamma_{\mu} t\right)\left(\bar{b} T^{a} \gamma^{\mu} d_{r}\right)$ & & & & \\
\hline \multicolumn{8}{|c|}{$\mathcal{L}_{6}^{(8 c)}-(\bar{L} L)(\bar{R} R)$} \\
\hline$Q_{l u}$ & $\left(\bar{l}_{p} \gamma_{\mu} l_{r}\right)\left(\bar{u} \gamma^{\mu} u\right)$ & $Q_{l d}$ & $\left(\bar{l}_{p} \gamma_{\mu} l_{r}\right)\left(\bar{d} \gamma^{\mu} d\right)$ & $Q_{q e}$ & $\left(\bar{q} \gamma_{\mu} q\right)\left(\bar{e}_{p} \gamma^{\mu} e_{r}\right)$ & $Q_{l e}$ & $\left(\bar{l}_{p} \gamma_{\mu} l_{r}\right)\left(\bar{e}_{s} \gamma^{\mu} e_{t}\right)$ \\
\hline$Q_{l t}$ & $\left(\bar{l}_{p} \gamma_{\mu} l_{r}\right)\left(\bar{t} \gamma^{\mu} t\right)$ & $Q_{l b}$ & $\left(\bar{l}_{p} \gamma_{\mu} l_{r}\right)\left(\bar{b} \gamma^{\mu} b\right)$ & $Q_{Q e}$ & $\left(\bar{Q} \gamma_{\mu} Q\right)\left(\bar{e}_{p} \gamma^{\mu} e_{r}\right)$ & & \\
\hline$Q_{q u}^{(1)}$ & $\left(\bar{q} \gamma_{\mu} q\right)\left(\bar{u} \gamma^{\mu} u\right)$ & $Q_{Q u}^{(1)}$ & $\left(\bar{Q} \gamma_{\mu} Q\right)\left(\bar{u} \gamma^{\mu} u\right)$ & $Q_{q t}^{(1)}$ & $\left(\bar{q} \gamma_{\mu} q\right)\left(\bar{t} \gamma^{\mu} t\right)$ & $Q_{Q t}^{(1)}$ & $\left(\bar{Q} \gamma_{\mu} Q\right)\left(\bar{t} \gamma^{\mu} t\right)$ \\
\hline$Q_{q u}^{(8)}$ & $\left(\bar{q} T^{a} \gamma_{\mu} q\right)\left(\bar{u} T^{a} \gamma^{\mu} u\right)$ & $Q_{Q u}^{(8)}$ & $\left(\bar{Q} T^{a} \gamma_{\mu} Q\right)\left(\bar{u} T^{a} \gamma^{\mu} u\right)$ & $Q_{q t}^{(8)}$ & $\left(\bar{q} T^{a} \gamma_{\mu} q\right)\left(\bar{t} T^{a} \gamma^{\mu} t\right)$ & $Q_{Q t}^{(8)}$ & $\left(\bar{Q} T^{a} \gamma_{\mu} Q\right)\left(\bar{t} T^{a} \gamma^{\mu} t\right)$ \\
\hline$Q_{q d}^{(1)}$ & $\left(\bar{q} \gamma_{\mu} q\right)\left(\bar{d} \gamma^{\mu} d\right)$ & $Q_{Q d}^{(1)}$ & $\left(\bar{Q} \gamma_{\mu} Q\right)\left(\bar{d} \gamma^{\mu} d\right)$ & $Q_{q b}^{(1)}$ & $\left(\bar{q} \gamma_{\mu} q\right)\left(\bar{b} \gamma^{\mu} b\right)$ & $Q_{Q b}^{(1)}$ & $\left(\bar{Q} \gamma_{\mu} Q\right)\left(\bar{b} \gamma^{\mu} b\right)$ \\
\hline$Q_{q d}^{(8)}$ & $\left(\bar{q} T^{a} \gamma_{\mu} q\right)\left(\bar{d} T^{a} \gamma^{\mu} d\right)$ & $Q_{Q d}^{(8)}$ & $\left(\bar{Q} T^{a} \gamma_{\mu} Q\right)\left(\bar{d} T^{a} \gamma^{\mu} d\right)$ & $Q_{q b}^{(8)}$ & $\left(\bar{q} T^{a} \gamma_{\mu} q\right)\left(\bar{b} T^{a} \gamma^{\mu} b\right)$ & $Q_{Q b}^{(8)}$ & $\left(\bar{Q} T^{a} \gamma_{\mu} Q\right)\left(\bar{b} T^{a} \gamma^{\mu} b\right)$ \\
\hline$Q_{q Q t u}^{(1)}$ & $\left(Y_{u}^{\dagger}\right)_{p r}\left(\bar{q}_{p} \gamma_{\mu} Q\right)\left(\bar{t} \gamma^{\mu} u_{r}\right)$ & $Q_{q Q t u}^{(8)}$ & $\left(Y_{u}^{\dagger}\right)_{p r}\left(\bar{q}_{p} T^{a} \gamma_{\mu} Q\right)\left(\bar{t} T^{a} \gamma^{\mu} u_{r}\right)$ & $Q_{q Q b d}^{(1)}$ & $\left(Y_{d}^{\dagger}\right)_{p r}\left(\bar{q}_{p} \gamma_{\mu} Q\right)\left(\bar{b} \gamma^{\mu} d_{r}\right)$ & $Q_{q Q b d}^{(8)}$ & $\left(Y_{d}^{\dagger}\right)_{p r}\left(\bar{q}_{p} T^{a} \gamma_{\mu} Q\right)\left(\bar{b} T^{a} \gamma^{\mu} d_{r}\right)$ \\
\hline \multicolumn{8}{|c|}{$\mathcal{L}_{6}^{(8 d)}-(\bar{L} R)(\bar{R} L),(\bar{L} R)(\bar{L} R)$} \\
\hline$Q_{l e d q}$ & $\left(\bar{l}_{p}^{j} e_{r}\right)\left(\bar{d} Y_{d} q_{j}\right)$ & $Q_{l e b Q}$ & $\left(\bar{l} j_{p}^{j} e_{r}\right)\left(\bar{b} Q_{j}\right)$ & $Q_{l e Q t}^{(1)}$ & $\left(\bar{l}_{p}^{j} e_{r}\right) \varepsilon_{j k}\left(\bar{Q}^{k} t\right)$ & $Q_{l e Q t}^{(3)}$ & $\left(\bar{l}_{p}^{j} \sigma_{\mu \nu} e_{r}\right) \varepsilon_{j k}\left(\bar{Q}^{k} \sigma^{\mu \nu} t\right)$ \\
\hline$Q_{\text {lequ }}^{(1)}$ & $\left(\bar{l}_{p}^{j} e_{r}\right) \varepsilon_{j k}\left(\bar{q}^{k} Y_{u}^{\dagger} u\right)$ & $Q_{\text {lequ }}^{(3)}$ & $\left(\bar{l}_{p}^{j} \sigma_{\mu \nu} e_{r}\right) \varepsilon_{j k}\left(\bar{q}^{k} Y_{u}^{\dagger} \sigma^{\mu \nu} u\right)$ & $Q_{Q t Q b}^{(1)}$ & $\left(\bar{Q}^{j} t\right) \varepsilon_{j k}\left(\bar{Q}^{k} b\right)$ & $Q_{Q t Q b}^{(8)}$ & $\left(\bar{Q}^{j} T^{a} t\right) \varepsilon_{j k}\left(\bar{Q}^{k} T^{a} b\right)$ \\
\hline$Q_{q u q d}^{(1)}$ & $\left(\bar{q}^{j} Y_{u}^{\dagger} u\right) \varepsilon_{j k}\left(\bar{q}^{k} Y_{d}^{\dagger} d\right)$ & $Q_{q u q d}^{(8)}$ & $\left(\bar{q}^{j} Y_{u}^{\dagger} T^{a} u\right) \varepsilon_{j k}\left(\bar{q}^{k} Y_{d}^{\dagger} T^{a} d\right)$ & $Q_{q u q d}^{(1) \prime}$ & $\left(Y_{u}^{\dagger}\right)_{s r}\left(Y_{d}^{\dagger}\right)_{p t}\left(\bar{q}_{p}^{j} u_{r}\right) \varepsilon_{j k}\left(\bar{q}_{s}^{k} d_{t}\right)$ & $Q_{q u q d}^{(8) \prime}$ & $\left(Y_{u}^{\dagger}\right)_{s r}\left(Y_{d}^{\dagger}\right)_{p t}\left(\bar{q}_{p}^{j} T^{a} u_{r}\right) \varepsilon_{j k}\left(\bar{q}_{s}^{k} T^{a} d_{t}\right)$ \\
\hline$Q_{Q t q d}^{(1)}$ & $\left(\bar{Q}^{j} t\right) \varepsilon_{j k}\left(\bar{q}^{k} Y_{d}^{\dagger} d\right)$ & $Q_{Q t q d}^{(8)}$ & $\left(\bar{Q}^{j} T^{a} t\right) \varepsilon_{j k}\left(\bar{q}^{k} Y_{d}^{\dagger} T^{a} d\right)$ & $Q_{q u Q b}^{(1)}$ & $\left(\bar{q}^{j} Y_{u}^{\dagger} u\right) \varepsilon_{j k}\left(\bar{Q}^{k} b\right)$ & $Q_{q u Q b}^{(8)}$ & $\left(\bar{q}^{j} Y_{u}^{\dagger} T^{a} u\right) \varepsilon_{j k}\left(\bar{Q}^{k} T^{a} b\right)$ \\
\hline$Q_{Q u q b}^{(1)}$ & $\left(Y_{u}^{\dagger}\right)_{p r}\left(\bar{Q}^{j} u_{r}\right) \varepsilon_{j k}\left(\bar{q}_{p}^{k} b\right)$ & $Q_{Q u q b}^{(8)}$ & $\left(Y_{u}^{\dagger}\right)_{p r}\left(\bar{Q}^{j} T^{a} u_{r}\right) \varepsilon_{j k}\left(\bar{q}_{p}^{k} T^{a} b\right)$ & $Q_{q t Q d}^{(1)}$ & $\left(Y_{d}^{\dagger}\right)_{p r}\left(\bar{q}_{p}^{j} t\right) \varepsilon_{j k}\left(\bar{Q}^{k} d_{r}\right)$ & $Q_{q t Q d}^{(8)}$ & $\left(Y_{d}^{\dagger}\right)_{p r}\left(\bar{q}_{p}^{j} T^{a} t\right) \varepsilon_{j k}\left(\bar{Q}^{k} T^{a} d_{r}\right)$ \\
\hline
\end{tabular}

Table 2. Basis of fermionic operators for the top and topU3l flavor assumptions. Here $(q, u, d)$, $Y_{u}, Y_{d}$ denote quarks of the first 2 generations and their $2 \times 2$ Yukawa matrices. Quark fields of the 3rd generation are $(Q, t, b)$. Flavor indices $p, r, s, t$ run over $\{1,2\}$ for light quarks and $\{1,2,3\}$ for leptons. Whenever flavor indices are not specified, they are implicitly contracted within each current. 
Class 7. Class 7 depends on 12 real parameters from quark operators, plus 9 (3) real parameters from lepton operators in the top (topU31) case. $Q_{H u d}$ is defined with a $Y_{u} Y_{d}^{\dagger}$ insertion to preserve $\mathrm{U}(2)^{3}$, while $Q_{H t b}$ is independent of the Yukawas.

$$
\begin{aligned}
\Lambda^{2} \mathcal{L}_{6}^{(7)}= & C_{H q}^{(1)} Q_{H q}^{(1)}+C_{H q}^{(3)} Q_{H q}^{(3)}+C_{H u} Q_{H u}+C_{H d} Q_{H d}+\left[C_{H u d} Q_{H u d}+\text { h.c. }\right] \\
& +C_{H Q}^{(1)} Q_{H Q}^{(1)}+C_{H Q}^{(3)} Q_{H Q}^{(3)}+C_{H t} Q_{H t}+C_{H b} Q_{H b}+\left[C_{H t b} Q_{H t b}+\text { h.c. }\right] \\
& +\left\{\begin{array}{ll}
\sum_{p=1}^{3}\left(C_{H l}^{(1)}\right)_{p p} Q_{H l, p p}^{(1)}+\left(C_{H l}^{(3)}\right)_{p p} Q_{H l, p p}^{(3)}+\left(C_{H e}\right)_{p p} Q_{H e, p p}, & \mathrm{U}(1)_{l+e}^{3}[\text { top }] \\
\sum_{p, r=1}^{3} C_{H l}^{(1)} \delta_{p r} Q_{H l, p r}^{(1)}+C_{H l}^{(3)} \delta_{p r} Q_{H l, p r}^{(3)}+C_{H e} \delta_{p r} Q_{H e, p r}, & \mathrm{U}(3)^{2}
\end{array}\right]
\end{aligned}
$$

Class 8 a. Class 8 a contains 2 operators with 4 quarks. Mapping them to the formalism with 6 quark fields, each of them admits 5 independent $\mathrm{U}(2)^{3}$ invariant contractions, that can be written

$$
\begin{array}{rlrl}
Q_{q q}^{(1)} & =\sum_{p, r, s, t=1}^{2} \delta_{p r} \delta_{s t}\left(\bar{q}_{p} \gamma_{\mu} q_{r}\right)\left(\bar{q}_{s} \gamma^{\mu} q_{t}\right), & Q_{Q q}^{(1)} & =\sum_{p, r=1}^{2} \delta_{p r}\left(\bar{q}_{p} \gamma_{\mu} q_{r}\right)\left(\bar{Q} \gamma^{\mu} Q\right), \\
Q_{q q}^{(1) \prime} & =\sum_{p, r, s, t=1}^{2} \delta_{p t} \delta_{s r}\left(\bar{q}_{p} \gamma_{\mu} q_{r}\right)\left(\bar{q}_{s} \gamma^{\mu} q_{t}\right), & Q_{Q q}^{(1) \prime}=\sum_{p, t=1}^{2} \delta_{p t}\left(\bar{q}_{p} \gamma_{\mu} Q\right)\left(\bar{Q} \gamma^{\mu} q_{t}\right) \\
Q_{Q Q}^{(1)} & =\left(\bar{Q} \gamma_{\mu} Q\right)\left(\bar{Q} \gamma^{\mu} Q\right)
\end{array}
$$

and analogously for $Q_{q q}^{(3)}$. In practice, for analyses involving top quark processes it is convenient to trade "crossed" flavor contractions, as well as $Q_{Q Q}^{(3)}$, for operators with a color octet structure. This is motivated by top processes being largely dominated by QCD interactions in the SM. The rotation is done using Fierz rearrangements and the completeness relations for $\mathrm{SU}(2)$ and $\mathrm{SU}(3)$

$$
\begin{aligned}
\sigma_{j k}^{i} \sigma_{m n}^{i} & =2 \delta_{j n} \delta_{m k}-\delta_{j k} \delta_{m n} \\
T_{a b}^{A} T_{c d}^{A} & =\frac{1}{2} \delta_{a d} \delta_{c b}-\frac{1}{6} \delta_{a b} \delta_{c d}
\end{aligned}
$$

Consistent with the recommendations in ref. [36], SMEFTsim implements the invariants in table 2, that are related to those in eqs. (3.48)-(3.50) and their $Q_{q q}^{(3)}$ counterparts as:

$$
\begin{array}{ll}
Q_{Q Q}^{(1)}=Q_{Q Q}^{(1)}, & Q_{Q Q}^{(3)}=-\frac{1}{3} Q_{Q Q}^{(1)}+4 Q_{Q Q}^{(8)}, \\
Q_{q q}^{(1)}=Q_{q q}^{(1,1)}, & Q_{q q}^{(1) \prime}=\frac{1}{6}\left(Q_{q q}^{(1,1)}+Q_{q q}^{(3,1)}\right)+Q_{q q}^{(1,8)}+Q_{q q}^{(3,8)}, \\
Q_{q q}^{(3)}=Q_{q q}^{(3,1)}, & Q_{q q}^{(3) \prime}=\frac{1}{2} Q_{q q}^{(1,1)}-\frac{1}{6} Q_{q q}^{(3,1)}+3 Q_{q q}^{(1,8)}-Q_{q q}^{(3,8)}, \\
Q_{Q q}^{(1)}=Q_{Q q}^{(1,1)}, & Q_{Q q}^{(1) \prime}=\frac{1}{6}\left(Q_{Q q}^{(1,1)}+Q_{Q q}^{(3,1)}\right)+Q_{Q q}^{(1,8)}+Q_{Q q}^{(3,8)}, \\
Q_{Q q}^{(3)}=Q_{Q q}^{(3,1)}, & Q_{Q q}^{(3) \prime}=\frac{1}{2} Q_{Q q}^{(1,1)}-\frac{1}{6} Q_{Q q}^{(3,1)}+3 Q_{Q q}^{(1,8)}-Q_{Q q}^{(3,8)} .
\end{array}
$$


Eqs. (3.53)-(3.57) can be written compactly as $\vec{Q}_{\text {Warsaw }}=R \vec{Q}_{\text {top }}$, with $\vec{Q}_{\text {Warsaw }}, \vec{Q}_{\text {top }}$ the two "operator vectors" and $R$ a rotation matrix. The relation among the Wilson coefficients is then derived equating the Lagrangian written in the two bases:

$$
\begin{aligned}
\mathcal{L} & =\vec{C}_{\text {Warsaw }} \cdot \vec{Q}_{\text {Warsaw }}=\left(\vec{C}_{\text {Warsaw }}\right)^{T} R \vec{Q}_{\text {top }} \\
& =\vec{C}_{\text {top }} \cdot \vec{Q}_{\text {top }}
\end{aligned}
$$

with $\vec{C}_{\text {Warsaw }}, \vec{C}_{\text {top }}$ the coefficients vectors. The solution is

$$
\vec{C}_{\text {top }}=R^{T} \vec{C}_{\text {Warsaw }}, \quad \vec{C}_{\text {Warsaw }}=\left(R^{T}\right)^{-1} \vec{C}_{\text {top }}
$$

Explicitly:

$$
\begin{aligned}
C_{Q Q}^{(1)} & =C_{Q Q}^{(1)}-\frac{1}{3} C_{Q Q}^{(3)}, & C_{Q Q}^{(8)} & =4 C_{Q Q}^{(3)}, \\
C_{q q}^{(1,1)} & =C_{q q}^{(1)}+\frac{1}{6} C_{q q}^{(1) \prime}+\frac{1}{2} C_{q q}^{(3) \prime}, & C_{q q}^{(3,1)} & =C_{q q}^{(3)}+\frac{1}{6} C_{q q}^{(1) \prime}-\frac{1}{6} C_{q q}^{(3) \prime}, \\
C_{q q}^{(1,8)} & =C_{q q}^{(1) \prime}+3 C_{q q}^{(3) \prime}, & C_{q q}^{(3,8)} & =C_{q q}^{(1) \prime}-C_{q q}^{(3) \prime}, \\
C_{Q q}^{(1,1)} & =C_{Q q}^{(1)}+\frac{1}{6} C_{Q q}^{(1) \prime}+\frac{1}{2} C_{Q q}^{(3) \prime}, & C_{Q q}^{(3,1)} & =C_{Q q}^{(3)}+\frac{1}{6} C_{Q q}^{(1) \prime}-\frac{1}{6} C_{Q q}^{(3) \prime}, \\
C_{Q q}^{(1,8)} & =C_{Q q}^{(1) \prime}+3 C_{Q q}^{(3) \prime}, & C_{Q q}^{(3,8)} & =C_{Q q}^{(1) \prime}-C_{Q q}^{(3) \prime},
\end{aligned}
$$

and the inverse

$$
\begin{aligned}
C_{Q Q}^{(1)} & =C_{Q Q}^{(1)}+\frac{1}{12} C_{Q Q}^{(8)}, & C_{Q Q}^{(3)} & =\frac{1}{4} C_{Q Q}^{(8)}, \\
C_{q q}^{(1)} & =C_{q q}^{(1,1)}-\frac{1}{6} C_{q q}^{(1,8)}, & C_{q q}^{(1) \prime} & =\frac{1}{4} C_{q q}^{(1,8)}+\frac{3}{4} C_{q q}^{(3,8)}, \\
C_{q q}^{(3)} & =C_{q q}^{(3,1)}-\frac{1}{6} C_{q q}^{(3,8)}, & C_{q q}^{(3) \prime} & =\frac{1}{4} C_{q q}^{(1,8)}-\frac{1}{4} C_{q q}^{(3,8)}, \\
C_{Q q}^{(1)} & =C_{Q q}^{(1,1)}-\frac{1}{6} C_{Q q}^{(1,8)}, & C_{Q q}^{(1) \prime} & =\frac{1}{4} C_{Q q}^{(1,8)}+\frac{3}{4} C_{Q q}^{(3,8)}, \\
C_{Q q}^{(3)} & =C_{Q q}^{(3,1)}-\frac{1}{6} C_{Q q}^{(3,8)}, & C_{Q q}^{(3) \prime} & =\frac{1}{4} C_{Q q}^{(1,8)}-\frac{1}{4} C_{Q q}^{(3,8)} .
\end{aligned}
$$

The operator $Q_{l l}$ admits 2 independent contractions in the $\mathrm{U}(3)^{2}$ symmetric case (eq. (3.20)) and 9 in the $\mathrm{U}(1)_{l+e}^{3}$ case. We choose them as the $\left(C_{l l}\right)_{\text {prst }}$ entries with indices prst in the set

$$
P_{l l}=\{1111,2222,3333,1122,1133,2233,1221,1331,2332\}
$$

Operators $Q_{l q}^{(1),(3)}, Q_{l Q}^{(1),(3)}$ admit 3 (1) contractions each in the top (topU31) case. 
The Lagrangian is therefore

$$
\begin{aligned}
\Lambda^{2} \mathcal{L}_{6}^{(8 a)}= & C_{q q}^{(1,1)} Q_{q q}^{(1,1)}+C_{q q}^{(1,8)} Q_{q q}^{(1,8)}+C_{q q}^{(3,1)} Q_{q q}^{(3,1)}+C_{q q}^{(3,8)} Q_{q q}^{(3,8)}+C_{Q Q}^{(1)} Q_{Q Q}^{(1)} \\
& +C_{Q q}^{(1,1)} Q_{Q q}^{(1,1)}+C_{Q q}^{(1,8)} Q_{Q q}^{(1,8)}+C_{Q q}^{(3,1)} Q_{Q q}^{(3,1)}+C_{Q q}^{(3,8)} Q_{Q q}^{(3,8)}+C_{Q Q}^{(8)} Q_{Q Q}^{(8)} \\
& +\left\{\begin{array}{l}
\sum_{p=1}^{3}\left(C_{l q}^{(1)}\right)_{p p} Q_{l q, p p}^{(1)}+\left(C_{l q}^{(3)}\right)_{p p} Q_{l q, p p}^{(3)}+\left(C_{l Q}^{(1)}\right)_{p p} Q_{l Q, p p}^{(1)}+\left(C_{l Q}^{(3)}\right)_{p p} Q_{l Q, p p}^{(3)} \mathrm{U}(1)_{l+e}^{3}[\mathrm{top}] \\
+\sum_{p r s t \in P_{l l}}\left(C_{l l}\right)_{p r s t} Q_{l l, p r s t}, \\
\sum_{p, r=1}^{3} C_{l q}^{(1)} \delta_{p r} Q_{l q, p r}^{(1)}+C_{l q}^{(3)} \delta_{p r} Q_{l q, p r}^{(3)}+C_{l Q}^{(1)} \delta_{p r} Q_{l Q, p r}^{(1)}+C_{l Q}^{(3)} \delta_{p r} Q_{l Q, p r}^{(3)} \mathrm{U}(3)^{2} \quad[\text { topU31] } \\
+\sum_{p, r, s, t=1}^{3}\left(C_{l l} \delta_{p r} \delta_{s t}+C_{l l}^{\prime} \delta_{p t} \delta_{s r}\right) Q_{l l, p r s t},
\end{array}\right.
\end{aligned}
$$

Note that the allowed flavor contractions in the $\mathrm{U}(1)_{l+r}^{3}$ and $\mathrm{U}(3)^{2}$ cases are the same, but the different symmetry properties generally lead to different relative normalizations. For instance, considering the (1111) and (1122) entries, one has

$$
\Lambda^{2} \mathcal{L}_{6}^{(8 a)} \supset \begin{cases}\left(C_{l l}\right)_{1111} Q_{1111}+\left(C_{l l}\right)_{1122} Q_{1122}+\ldots & \mathrm{U}(1)_{l+e}^{3}[\text { top }] \\ \left(C_{l l}+C_{l l}^{\prime}\right) Q_{1111}+2 C_{l l} Q_{1122}+\ldots & \mathrm{U}(3)^{2} \quad \text { [topU31] }\end{cases}
$$

where the relative 2 between the $C_{l l}$ contributions to $Q_{1122}$ and $Q_{1111}$ is due to $\mathrm{U}(3)^{2}$ requiring to sum over both the 1122 and 2211 contractions, that are equivalent for this particular operator. In total, $\mathcal{L}_{6}^{(8 a)}$ contains 31 independent real parameters in the top case and 16 in the topU31 case.

Class 8 b. A basis rotation analogous to the one performed in $\mathcal{L}_{6}^{(8 a)}$ is applied to $Q_{u u}, Q_{u d}$ in $\mathcal{L}_{6}^{(8 b)}$. No modification is needed for $Q_{u d}^{(1),(8)}$ as in this case the color octet contraction is already manifest. The set of 5 independent $\mathrm{U}(2)^{3}$-invariant contractions in the Warsaw basis is in this case

$$
\begin{aligned}
Q_{u u} & =\sum_{p, r, s, t=1}^{2} \delta_{p r} \delta_{s t}\left(\bar{u}_{p} \gamma_{\mu} u_{r}\right)\left(\bar{u}_{s} \gamma^{\mu} u_{t}\right), & Q_{t u} & =\sum_{p, r=1}^{2} \delta_{p r}\left(\bar{u}_{p} \gamma_{\mu} u_{r}\right)\left(\bar{t} \gamma^{\mu} t\right), \\
Q_{u u}^{\prime} & =\sum_{p, r, s, t=1}^{2} \delta_{p t} \delta_{s r}\left(\bar{u}_{p} \gamma_{\mu} u_{r}\right)\left(\bar{u}_{s} \gamma^{\mu} u_{t}\right), & Q_{t u}^{\prime} & =\sum_{p, t=1}^{2} \delta_{p t}\left(\bar{u}_{p} \gamma_{\mu} t\right)\left(\bar{t} \gamma^{\mu} u_{t}\right), \\
Q_{t t} & =\left(\bar{t} \gamma_{\mu} t\right)\left(\bar{t} \gamma^{\mu} t\right), & &
\end{aligned}
$$

and analogously for the $Q_{d d}$ counterparts. Using Fierz transformations and eqs. (3.51), (3.52):

$$
\begin{aligned}
Q_{t t} & =Q_{t t}, & Q_{b b} & =Q_{b b}, \\
Q_{u u} & =Q_{u u}^{(1)}, & Q_{u u}^{\prime} & =\frac{1}{3} Q_{u u}^{(1)}+2 Q_{u u}^{(8)}, \\
Q_{d d} & =Q_{d d}^{(1)}, & Q_{d d}^{\prime} & =\frac{1}{3} Q_{d d}^{(1)}+2 Q_{d d}^{(8)}, \\
Q_{t u} & =Q_{t u}^{(1)}, & Q_{t u}^{\prime} & =\frac{1}{3} Q_{t u}^{(1)}+2 Q_{t u}^{(8)}, \\
Q_{b d} & =Q_{b d}^{(1)}, & Q_{b d}^{\prime} & =\frac{1}{3} Q_{b d}^{(1)}+2 Q_{b d}^{(8)},
\end{aligned}
$$


where the operators on the right-hand side of the equations are defined in table 2. The relations among Wilson coefficients are

$$
\begin{aligned}
C_{t t} & =C_{t t}, & C_{b b} & =C_{b b}, \\
C_{u u}^{(1)} & =C_{u u}+\frac{1}{3} C_{u u}^{\prime}, & C_{u u}^{(8)} & =2 C_{u u}^{\prime}, \\
C_{d d}^{(1)} & =C_{d d}+\frac{1}{3} C_{d d}^{\prime}, & C_{d d}^{(8)} & =2 C_{d d}^{\prime}, \\
C_{t u}^{(1)} & =C_{t u}+\frac{1}{3} C_{t u}^{\prime}, & C_{t u}^{(8)} & =2 C_{t u}^{\prime}, \\
C_{b d}^{(1)} & =C_{b d}+\frac{1}{3} C_{b d}^{\prime}, & C_{b d}^{(8)} & =2 C_{b d}^{\prime},
\end{aligned}
$$

and the inverse

$$
\begin{aligned}
C_{t t} & =C_{t t}, & C_{b b} & =C_{b b}, \\
C_{u u} & =C_{u u}^{(1)}-\frac{1}{6} C_{u u}^{(8)}, & C_{u u}^{\prime} & =\frac{1}{2} C_{u u}^{(8)}, \\
C_{d d} & =C_{d d}^{(1)}-\frac{1}{6} C_{d d}^{(8)}, & C_{d d}^{\prime} & =\frac{1}{2} C_{d d}^{(8)}, \\
C_{t u} & =C_{t u}^{(1)}-\frac{1}{6} C_{t u}^{(8)}, & C_{t u}^{\prime} & =\frac{1}{2} C_{t u}^{(8)}, \\
C_{b d} & =C_{b d}^{(1)}-\frac{1}{6} C_{b d}^{(1)}, & C_{b d}^{\prime} & =\frac{1}{2} C_{b d}^{(8)} .
\end{aligned}
$$

The operator $Q_{e e}$ admits 6 independent contractions in the top case, with indices that we choose in the set

$$
P_{e e}=\{1111,2222,3333,1122,1133,2233\} .
$$

In the $\mathrm{U}(3)^{2}$ case, there is instead only 1 available contraction. Each of the operators $Q_{l u}^{(1),(3)}, Q_{l d}^{(1),(3)}$ is mapped into 6 (2) independent invariants in the top (topU31) case. The Lagrangian for class 8 a has the form

$$
\begin{aligned}
\Lambda^{2} \mathcal{L}_{6}^{(8 b)}= & C_{u u}^{(1)} Q_{u u}^{(1)}+C_{u u}^{(8)} Q_{u u}^{(8)}+C_{t t} Q_{t t}+C_{t u}^{(1)} Q_{t u}^{(1)}+C_{t u}^{(8)} Q_{t u}^{(8)} \\
& +C_{d d}^{(1)} Q_{d d}^{(1)}+C_{d d}^{(8)} Q_{d d}^{(8)}+C_{b b} Q_{b b}+C_{b d}^{(1)} Q_{b d}^{(1)}+C_{b d}^{(8)} Q_{b d}^{(8)} \\
& +C_{u d}^{(1)} Q_{u d}^{(1)}+C_{u d}^{(8)} Q_{u d}^{(8)}+C_{t d}^{(1)} Q_{t d}^{(1)}+C_{t d}^{(8)} Q_{t d}^{(8)}+C_{u b}^{(1)} Q_{u b}^{(1)}+C_{u b}^{(8)} Q_{u b}^{(8)}+C_{t b}^{(1)} Q_{t b}^{(1)}+C_{t b}^{(8)} Q_{t b}^{(8)} \\
& +\left[\begin{array}{ll}
\left.C_{u t b d}^{(1)} Q_{u t b d}^{(1)}+C_{u t b d}^{(8)} Q_{u t b d}^{(8)}+\text { h.c. }\right] \\
+
\end{array}\right. \\
& +\left\{\begin{array}{ll}
\sum_{p=1}^{3}\left(C_{e u}\right)_{p p} Q_{e u, p p}+\left(C_{e d}\right)_{p p} Q_{e d, p p}+\left(C_{e t}\right)_{p p} Q_{e t, p p}+\left(C_{e b}\right)_{p p} Q_{e b, p p} \\
+\sum_{p r s t \in P_{e e}}\left(C_{e e}\right)_{p r s t} Q_{e e, p r s t}, \\
\sum_{p, r=1}^{3} C_{e u} \delta_{p r} Q_{e u, p r}+C_{e d} \delta_{p r} Q_{e d, p r}+C_{e t} \delta_{p r} Q_{e t, p r}+C_{e b} \delta_{p r} Q_{e b, p r} & \mathrm{U}(1)_{l+e}^{3}[\text { top] } \\
+\sum_{p, r, s, t=1}^{3} C_{e e} \delta_{p r} \delta_{s t} Q_{e e, p r s t}, & \mathrm{U}(3)^{2}
\end{array}\right] \text { [topU31] }
\end{aligned}
$$

and it depends on 40 (27) real independent parameters in the top (topU31) case.

Class 8 c. No basis rotation is required in $\mathcal{L}_{6}^{(8 c)}$, and the quark currents are mapped directly. In the lepton sector, $Q_{l e}$ admits 1 independent contraction in the $\mathrm{U}(3)^{2}$ case 
(neglecting the subleading contribution $\propto Y_{l}^{2}$ ) and 12 in the $\mathrm{U}(1)_{l+e}^{3}$ case. We choose those with indices prst in the set

$$
\begin{aligned}
& P_{l e}=P_{l e}^{h} \cup P_{l e}^{n h}, \\
& P_{l e}^{h}=\{1111,2222,3333,1122,1133,2233,2211,3311,3322\}, \quad P_{l e}^{n h}=\{1221,1331,2332\},
\end{aligned}
$$

where the contractions in $P_{l e}^{h}$ are Hermitian and those in $P_{l e}^{n h}$ are not. The operators $Q_{q Q t u}^{(1),(8)}, Q_{q Q b d}^{(1),(8)}$ are not Hermitian and therefore the associated Wilson coefficients are complex.

The Lagrangian reads

$$
\begin{aligned}
& \Lambda^{2} \mathcal{L}_{6}^{(8 c)}=C_{q u}^{(1)} Q_{q u}^{(1)}+C_{q t}^{(1)} Q_{q t}^{(1)}+C_{Q u}^{(1)} Q_{Q u}^{(1)}+C_{Q t}^{(1)} Q_{Q t}^{(1)} \\
& +C_{q u}^{(8)} Q_{q u}^{(8)}+C_{q t}^{(8)} Q_{q t}^{(8)}+C_{Q u}^{(8)} Q_{Q u}^{(8)}+C_{Q t}^{(8)} Q_{Q t}^{(8)} \\
& +C_{q d}^{(1)} Q_{q d}^{(1)}+C_{q b}^{(1)} Q_{q b}^{(1)}+C_{Q d}^{(1)} Q_{Q d}^{(1)}+C_{Q b}^{(1)} Q_{Q b}^{(1)} \\
& +C_{q d}^{(8)} Q_{q d}^{(8)}+C_{q b}^{(8)} Q_{q b}^{(8)}+C_{Q d}^{(8)} Q_{Q d}^{(8)}+C_{Q b}^{(8)} Q_{Q b}^{(8)} \\
& +\left[C_{q Q t u}^{(1)} Q_{q Q t u}^{(1)}+C_{q Q t u}^{(8)} Q_{q Q t u}^{(8)}+C_{q Q b d}^{(1)} Q_{q Q b d}^{(1)}+C_{q Q b d}^{(8)} Q_{q Q b d}^{(8)}+\text { h.c. }\right] \\
& +\left\{\begin{aligned}
\sum_{p=1}^{3} & \left(C_{l u}\right)_{p p} Q_{l u, p p}+\left(C_{l d}\right)_{p p} Q_{l d, p p}+\left(C_{l t}\right)_{p p} Q_{l t, p p}+\left(C_{l b}\right)_{p p} Q_{l b, p p} \\
& +\left(C_{q e}\right)_{p p} Q_{q e, p p}+\left(C_{Q e}\right)_{p p} Q_{Q e, p p}+\sum_{p r s t \in P_{l e}^{h}}\left(C_{l e}\right)_{p r s t} Q_{l e, p r s t} \quad \mathrm{U}(1)_{l+e}^{3}[\mathrm{top}] \\
& +\left[\sum_{p r s t \in P_{l e}^{n h}}\left(C_{l e}\right)_{p r s t} Q_{l e, p r s t}+\text { h.c. }\right], \\
\sum_{p, r=1}^{3} & C_{l u} \delta_{p r} Q_{l u, p r}+C_{l d} \delta_{p r} Q_{l d, p r}+C_{l t} \delta_{p r} Q_{l t, p r}+C_{l b} \delta_{p r} Q_{l b, p r} \\
& +C_{q e} \delta_{p r} Q_{q e, p r}+C_{Q e} \delta_{p r} Q_{Q e, p r}+\sum_{p, r, s, t=1}^{3} C_{l e} \delta_{p r} \delta_{s t} Q_{l e, p r s t}, \quad \mathrm{U}(3)^{2} \quad \text { [topU31] }
\end{aligned}\right.
\end{aligned}
$$

and it depends on 54 (31) independent real parameters in the top (topU31) case.

Class 8 d. Finally, the operators in $\mathcal{L}_{6}^{(8 d)}$ are also mapped directly to the notation with 6 quark fields. $\mathrm{U}(2)^{3}$ invariance requires an insertion of a light Yukawa couplings for each $(\bar{q} u)$ or $(\bar{q} d)$ current and an insertion of $Y_{l}$ for each $(\bar{l} e)$ current, as indicated in table 2.

This class includes a total of 64 (40) real parameters in the top (topU31) case:

$$
\begin{aligned}
& \Lambda^{2} \mathcal{L}_{6}^{(8 d)}=C_{\text {quqd }}^{(1)} Q_{\text {quqd }}^{(1)}+C_{\text {quqd }}^{(8)} Q_{\text {quqd }}^{(8)}+C_{\text {quqd }}^{(1) \prime} Q_{\text {quqd }}^{(1) \prime}+C_{\text {quqd }}^{(8) \prime} Q_{\text {quqd }}^{(8) \prime}+C_{Q t Q b}^{(1)} Q_{Q t q b}^{(1)}+C_{Q t Q b}^{(8)} Q_{Q t q b}^{(8)} \\
& +C_{Q t q d}^{(1)} Q_{Q t q b}^{(1)}+C_{q u Q b}^{(1)} Q_{q u Q b}^{(1)}+C_{Q u q b}^{(1)} Q_{Q u q b}^{(1)}+C_{q t Q d}^{(1)} Q_{q t Q d}^{(1)} \\
& +C_{Q t q d}^{(8)} Q_{Q t q b}^{(8)}+C_{q u Q b}^{(8)} Q_{q u Q b}^{(8)}+C_{Q u q b}^{(8)} Q_{Q u q b}^{(8)}+C_{q t Q d}^{(8)} Q_{q t Q d}^{(8)} \\
& + \begin{cases}\sum_{p=1}^{3}\left(C_{l e q u}^{(1)}\right)_{p p} Q_{l e q u, p p}^{(1)}+\left(C_{l e Q t}^{(1)}\right)_{p p} Q_{l e Q t, p p}^{(1)}+\left(C_{l e q u}^{(3)}\right)_{p p} Q_{l e q u, p p}^{(3)} & \\
\quad+\left(C_{l e Q t}^{(3)}\right)_{p p} Q_{l e Q t, p p}^{(3)}+\left(C_{l e d q}\right)_{p p} Q_{l e d q, p p}+\left(C_{l e b Q}\right)_{p p} Q_{l e b Q, p p}, & \mathrm{U}(1)_{l+e}^{3}[\mathrm{top}] \\
\sum_{p, r=1}^{3} C_{l e q u}^{(1)}\left(Y_{l}^{\dagger}\right)_{p r} Q_{l e q u, p r}^{(1)}+C_{l e Q t}^{(1)}\left(Y_{l}^{\dagger}\right)_{p r} Q_{l e Q t, p r}^{(1)}+C_{l e q u}^{(3)}\left(Y_{l}^{\dagger}\right)_{p r} Q_{l e q u, p r}^{(3)} & \\
\quad+C_{l e Q t}^{(3)}\left(Y_{l}^{\dagger}\right)_{p r} Q_{l e Q t, p r}^{(3)}+C_{l e d q}\left(Y_{l}^{\dagger}\right)_{p r} Q_{l e d q, p r}+C_{l e b Q}\left(Y_{l}^{\dagger}\right)_{p r} Q_{l e b Q, p r}, & \mathrm{U}(3)^{2} \quad \text { [topU31] }\end{cases} \\
& \text { +h.c. }
\end{aligned}
$$

\subsection{Comparison with the literature}

We conclude this section with a comparison of the parameterizations presented in this section with other recent results in the literature. As a quantitative reference, table 3 


\begin{tabular}{|c|cc|cc|cc|cc|cc|}
\hline & \multicolumn{2}{|c|}{ general } & \multicolumn{2}{|c|}{ U35 } & \multicolumn{2}{c|}{ MFV } & \multicolumn{2}{c|}{ top } & \multicolumn{2}{c|}{ topU31 } \\
\cline { 2 - 12 } & all & CP & all & CP & all & CP & all & CP & all & CP \\
\hline $\mathcal{L}_{6}^{(1)}$ & 4 & 2 & 4 & 2 & 2 & - & 4 & 2 & 4 & 2 \\
$\mathcal{L}_{6}^{(2,3)}$ & 3 & - & 3 & - & 3 & - & 3 & - & 3 & - \\
$\mathcal{L}_{6}^{(4)}$ & 8 & 4 & 8 & 4 & 4 & - & 8 & 4 & 8 & 4 \\
\hline $\mathcal{L}_{6}^{(5)}$ & 54 & 27 & 6 & 3 & 7 & - & 14 & 7 & 10 & 5 \\
$\mathcal{L}_{6}^{(6)}$ & 144 & 72 & 16 & 8 & 20 & - & 36 & 18 & 28 & 14 \\
$\mathcal{L}_{6}^{(7)}$ & 81 & 30 & 9 & 1 & 14 & - & 21 & 2 & 15 & 2 \\
$\mathcal{L}_{6}^{(8 a)}$ & 297 & 126 & 8 & - & 10 & - & 31 & - & 16 & - \\
$\mathcal{L}_{6}^{(8 b)}$ & 450 & 195 & 9 & - & 19 & - & 40 & 2 & 27 & 2 \\
$\mathcal{L}_{6}^{(8 c)}$ & 648 & 288 & 8 & - & 28 & - & 54 & 4 & 31 & 4 \\
$\mathcal{L}_{6}^{(8 d)}$ & 810 & 405 & 14 & 7 & 13 & - & 64 & 32 & 40 & 20 \\
\hline tot & 2499 & 1149 & 85 & 25 & 120 & - & 275 & 71 & 182 & 53 \\
\hline
\end{tabular}

Table 3. Number of independent real parameters in each class of dimension 6 operators, for the 5 flavor structures implemented in SMEFTsim.

summarizes the number of independent real parameters for each class of $\mathcal{L}_{6}$ operators and flavor setup.

Compared to previous versions of SMEFTsim [1], the following changes were made:

- the dependence on the CKM matrix in currents involving left-handed down quarks was neglected in the effective operators defined in the general and MFV versions. It has been restored in version 3.0.

- four parameters corresponding to the real and imaginary parts of $C_{q u q d}^{(1) \prime}, C_{q u q d}^{(8) \prime}$ were missing in the U35 and MFV models, and have now been included.

- the MFV models have been modified: all Yukawas are now retained in the spurions, instead of only $y_{t}, y_{b}$. Moreover, the Lagrangian is now organized according to a power counting in the quark Yukawas, that led to some flavor-violating terms (eg. $\left.\Delta^{u} C_{H u d}, \Delta_{1}^{u} C_{q u q d}^{(1)} \ldots\right)$ being dropped, and others (eg. $\left.\Delta^{d} C_{u H}, C_{q u}^{(1) \prime} \ldots\right)$ being added.

- versions top and topU31 are new in version 3.0.

The U35 and MFV models can be compared, for instance, to the $\mathrm{U}(3)^{5}$ spurion analyses presented in refs. [66, 75]. Ref. [75] contains an exhaustive classification of all the flavor spurions associated with SM fermion currents in the presence of a $\mathrm{U}(3)^{5}$ symmetry. In their notation, $S^{u}, S^{d}$ correspond to $\Delta_{U}, \Delta_{D}$ respectively, while both $S^{q u}, S^{q d}$ are mapped to $\Delta_{Q}$. The structure $Y_{u} Y_{d}^{\dagger}$ corresponds to $\Delta_{U D}$ and, since we only retain linear insertions of $Y_{l}$, $\Delta_{L}=\Delta_{E}=0$ in SMEFTsim. Any other spurion leads to baryon and/or lepton number nonconservation, and therefore does not have an equivalent in the Lagrangian considered here. 
Ref. [66] presented a detailed classification of all the $\mathrm{U}(3)^{5}$ and $\mathrm{U}(2)^{5}$ invariant structures in the Warsaw basis. In the $\mathrm{U}(3)^{5}$ case, their results can be directly compared with the parameterizations of the U35 and MFV models in SMEFTsim, while the U(2) ${ }^{3}$ case can be compared (in the quark sector) to the top and topU31 models. We find complete agreement in the characterization of the structures, and the operator countings are consistent once a few differences in the organization of the invariants are taken into account:

- the $\mathrm{U}(3)^{5}$ and $\mathrm{U}(2)^{5}$ Lagrangians in ref. [66] are organized according to a power counting in the Yukawas, while for the U35, top and topU31 models in SMEFTsim we simply choose to retain the leading invariant for each operator in the Warsaw basis.

- in the MFV models we retain terms up to order $\left(Y_{l}^{1},\left(Y_{d}+Y_{u}\right)^{3}\right)$. This choice is different from the power counting in ref. [66], that truncates at $\left(Y_{l}^{1}, Y_{d}^{1} Y_{u}^{2}\right)$.

- the Lagrangian of the MFV models includes spurions $\propto Y_{d}^{2}$, that were neglected in ref. [66].

- in the $\mathrm{U}(2)$ case, different symmetries were chosen for the lepton sector: $\mathrm{U}(2)^{3}$ in ref. [66] vs $\mathrm{U}(1)_{l+e}^{3}$ and $\mathrm{U}(3)^{2}$ in the top and topU3l models.

The structure of the top and topU31 versions builds upon those of refs. $[7,9,36]$. The main difference compared to these works is that in SMEFTsim the parameterization has been systematically extended to all operators of the Warsaw basis, including at the same time $\mathrm{CP}$ violating terms, interactions that do not involve the top quark, and spurion insertions of the light quark Yukawas.

\section{Input parameters}

Once the kinetic terms have been canonically normalized and the flavor structure has been fixed, the Lagrangian parameters can be assigned numerical values, with a procedure that is sometimes referred to as "fixing an input parameter scheme" or "finite renormalization". This section revisits this procedure in the SM and in the SMEFT, using a general formalism that accounts for terms up to arbitrary EFT order. They can be applied to both tree level and loop calculations but, in the latter case, this procedure needs to be combined with the usual renormalization to reabsorb UV divergences. In sections 4.2, 4.3 these formulas are applied to the Warsaw basis case, to recover the known tree-level results, see e.g. $[1,2,48,52,63,76,77]$. Aspects specific to the NLO case have been discussed in $[51,53,54,56,78,79]$.

The Lagrangian parameters are fixed imposing a set of defining conditions that relate them to (pseudo-)observables: for a Lagrangian with $N$ independent parameters $g=\left\{g_{i} \ldots g_{N}\right\}, M \geq N$ independent input observables $\mathcal{O}=\left\{\mathcal{O}_{1} \ldots \mathcal{O}_{M}\right\}$ need to be selected. Computing each $\mathcal{O}_{n}$ in the theory at a chosen perturbative order, one obtains relations

$$
\mathcal{O}_{n}=F_{n}^{(0)}(g), \quad n=1 \ldots M
$$


where $F_{n}^{(0)}$ denotes a function of the parameters $g$. If $M=N$ and (4.1) is an invertible system of equations, the solution

$$
g_{i}=K_{i}^{(0)}(\mathcal{O}), \quad i=1 \ldots N
$$

fixes $g_{i}$ as a function $K_{i}^{(0)}$ of $\mathcal{O}$. The numerical values of the parameters $g$ are then univocally determined by measurements of $\mathcal{O}$.

In the SM case, one has 19 independent parameters, that we can classify as

$$
\begin{array}{ll}
\alpha_{s}, \bar{\theta}, & \text { QCD } \\
g_{1}, g_{W}, v, \lambda, & \text { EW+Higgs } \\
y_{e}, y_{\mu}, y_{\tau}, y_{u}, y_{c}, y_{t}, y_{d}, y_{s}, y_{b}, & \text { Yukawas } \\
\theta_{12}, \theta_{13}, \theta_{23}, \delta, & \text { CKM }
\end{array}
$$

where we have introduced the CP-violating $\theta$ angle of QCD $(\bar{\theta})$ and the CKM angles and CP phase $\left(\theta_{12}, \theta_{13}, \theta_{23}\right.$ and $\delta$ respectively). The procedure outlined above is most often employed to determine the value of $\bar{\theta}$ and of the EW+Higgs and Yukawa parameters. On the other hand, the determination of the CKM parameters and of $\alpha_{s}$ usually relies on a large number of observables: in these cases, the system (4.1) is not invertible and the parameters' values are extracted via a global fit.

When transitioning from the SM to the SMEFT, a large number of additional parameters enters the Lagrangian, namely the cutoff $\Lambda$ and the Wilson coefficients $C_{\alpha}$. Fixing their numerical values in terms of measured observables is obviously still an open challenge (and indeed the ultimate goal of the present work), so these quantities are necessarily left free in the Lagrangian. Nevertheless, they play a role in the finite renormalization procedure, because the observables $\mathcal{O}$ employed to fix the SM quantities generically receive contributions from higher dimensional operators. Working order by order in the EFT expansion, the relations in (4.1) are modified into ${ }^{9}$

$$
\mathcal{O}_{n}=F_{n}^{(0)}(g)+\frac{1}{\Lambda^{2}} F_{n}^{(2)}(g, C)+\frac{1}{\Lambda^{4}} F_{n}^{(4)}(g, C)+\ldots
$$

where $C$ here generically represents the set of relevant Wilson coefficients, that can be associated to operators of any dimension. In cases where the system of eq. (4.1) can be inverted, (4.4) can also be solved expanding around the SM solution. The result has the general form:

$$
g_{i}=K_{i}^{(0)}(\mathcal{O})+\frac{1}{\Lambda^{2}} K_{i}^{(2)}(\mathcal{O}, C)+\frac{1}{\Lambda^{4}} K_{i}^{(4)}(\mathcal{O}, C)+\ldots
$$

where $K_{i}^{(0)}(\mathcal{O})$ is the SM solution and the following $K$ terms are SMEFT corrections that depend on the Wilson coefficients. The leading term in the solution (4.5) is defined imposing that the SM relation holds:

$$
\mathcal{O}_{n} \equiv F_{n}^{(0)}\left(K^{(0)}(\mathcal{O})\right)
$$

\footnotetext{
${ }^{9}$ Terms suppressed by odd powers of $\Lambda$ are omitted here, as they typically contribute to $B$ and/or $L$ violating observables, that are not relevant for the extraction of SM parameters. Nevertheless, the results derived in this section directly generalize to the case where these contributions are retained.
} 
The explicit form of the remaining $K$ terms is found inserting eq. (4.5) into (4.4), expanding in $\Lambda$ and requiring that SMEFT corrections cancel order by order in the resulting expression. Iteratively, one finds

$$
\begin{aligned}
K_{i}^{(2)} & =-\left(J^{-1}\right)_{i n} F_{n}^{(2)}, \\
K_{i}^{(4)} & =-\left(J^{-1}\right)_{i n}\left[F_{n}^{(4)}+\frac{\partial F_{n}^{(2)}}{\partial g_{k}} K_{k}^{(2)}+\frac{1}{2} \frac{\partial^{2} F_{n}^{(0)}}{\partial g_{k} \partial g_{j}} K_{k}^{(2)} K_{j}^{(2)}\right], \\
& \vdots \\
K_{i}^{(d)} & =-\left(J^{-1}\right)_{i n}\left[F_{n}^{(d)}+\sum_{\substack{m<d \\
m+d_{1}+\ldots d_{D}=d}} \frac{1}{D !} \frac{\partial^{D} F_{n}^{(m)}}{\partial g_{i_{1}} \cdots \partial g_{i_{D}}} K_{i_{1}}^{\left(d_{1}\right)} \cdots K_{i_{D}}^{\left(d_{D}\right)}\right],
\end{aligned}
$$

where $\left(J^{-1}\right)_{\text {in }}=\partial g_{i} / \partial F_{n}^{(0)}$ is the inverse of the Jacobian matrix

$$
J_{n i}=\frac{\partial F_{n}^{(0)}}{\partial g_{i}},
$$

and in eq. (4.9) the sum runs over all possible terms with $m<d$ and such that $m+$ $d_{1}+\cdots+d_{D}=d$. All functions and derivatives appearing explicitly in eqs. (4.7)-(4.9) are evaluated at the SM solution for the parameters $g \equiv K^{(0)}(\mathcal{O})$ and the indices $n, k, j, i_{1} \ldots i_{D}$ are implicitly contracted internally and summed over.

A generic predicted observable $\mathcal{P}$ inherits a dependence on the corrections $F_{n}^{(d \geq 2)}$ to the input quantities. Analogous to $\mathcal{O}, \mathcal{P}$ will have the generic form

$$
\mathcal{P}=P^{(0)}(g)+\frac{1}{\Lambda^{2}} P^{(2)}(g, C)+\frac{1}{\Lambda^{4}} P^{(4)}(g, C)+\ldots
$$

where $P^{(0)}$ is the SM expression and $P^{(d \geq 2)}$ encode direct EFT contributions to $\mathcal{P}$, induced by effective operators entering the relevant Feynman diagrams. Calculating $\mathcal{P}$ in the SMEFT starting from input quantities $\mathcal{O}$ means inserting the expressions of $g$ in eq. (4.5) into eq. (4.11). This operation introduces "indirect" EFT contributions, that are a direct consequence of the $F_{n}^{(d \geq 2)}$ terms in eq. (4.4). The dependence on the latter quantities can be made explicit:

$$
\mathcal{P}=P^{(0)}+\frac{1}{\Lambda^{2}}\left[P^{(2)}-A_{n} F_{n}^{(2)}\right]+\frac{1}{\Lambda^{4}}\left[P^{(4)}-A_{n} F_{n}^{(4)}-A_{n}^{(2)} F_{n}^{(2)}+B_{m n} F_{m}^{(2)} F_{n}^{(2)}\right]+\ldots
$$

where the $m, n$ indices are summed over and, as above, all functions are implicitly evaluated at $g \equiv K^{(0)}(\mathcal{O})$. The coefficients $A, B$ are found via chain differentiation:

$$
\begin{aligned}
A_{n} & =\frac{\partial P^{(0)}}{\partial F_{n}^{(0)}}=\frac{\partial P^{(0)}}{\partial g_{i}}\left(J^{-1}\right)_{i n}, \\
A_{n}^{(2)} & =\frac{\partial P^{(2)}}{\partial F_{n}^{(0)}}-\frac{\partial P^{(0)}}{\partial F_{m}^{(0)}} \frac{\partial F_{m}^{(2)}}{\partial F_{n}^{(0)}}=\frac{\partial P^{(2)}}{\partial g_{i}}\left(J^{-1}\right)_{i n}-A_{m} \frac{\partial F_{m}^{(2)}}{\partial g_{k}}\left(J^{-1}\right)_{k n}, \\
B_{m n} & =\frac{1}{2} \frac{\partial^{2} P^{(0)}}{\partial F_{m}^{(0)} \partial F_{n}^{(0)}}-\frac{1}{2} \frac{\partial P^{(0)}}{\partial F_{p}^{(0)}} \frac{\partial^{2} F_{p}^{(0)}}{\partial F_{m}^{(0)} \partial F_{n}^{(0)}}= \\
& =\frac{1}{2} \frac{\partial^{2} P^{(0)}}{\partial g_{i} \partial g_{j}}\left(J^{-1}\right)_{i m}\left(J^{-1}\right)_{j n}-\frac{1}{2} A_{p} \frac{\partial^{2} F_{p}^{(0)}}{\partial g_{i} \partial g_{j}}\left(J^{-1}\right)_{i m}\left(J^{-1}\right)_{j n} .
\end{aligned}
$$


Here $A_{n}$ and the first term in $A_{n}^{(2)}$ account for linear $K^{(2)}$ corrections to $g$ in the $P^{(0)}$ and $P^{(2)}$ function respectively. The first term in $B_{m n}$ contains double $K^{(2)}$ insertions ${ }^{10}$ in $P^{(0)}$, while the second terms of $A_{n}^{(2)}$ and $B_{m n}$ both stem from $K^{(4)}$ contributions in $P^{(0)}$.

The net effect of the finite renormalization procedure is that all the EFT corrections to input measurements are recast into corrections to predicted quantities: if $\mathcal{P}$ is an input observable $\mathcal{P} \equiv \mathcal{O}_{q}$, all EFT corrections in eq. (4.12) cancel order by order in the EFT. This happens by construction and follows trivially from the defining conditions imposed. It can be checked explicitly: in this case $P^{(d)}=F_{q}^{(d)}$ and assuming that $\mathcal{O}$ is a set of independent quantities, also $\partial F_{q}^{(0)} / \partial F_{n}^{(0)}=\delta_{q n}$ and $\partial^{2} F_{q}^{(0)} / \partial F_{m}^{(0)} \partial F_{n}^{(0)}=0$. This immediately leads to

$$
\begin{aligned}
& P^{(2)}-A_{n} F_{n}^{(2)}=0, \\
& B_{m n}=0, \\
& A_{n}^{(2)}=0, \\
& P^{(4)}-A_{n} F_{n}^{(4)}=0 \text {. }
\end{aligned}
$$

Eq. (4.12) provides a dictionary between different input parameter schemes: comparing sets $\mathcal{O}$ and $\mathcal{O}^{\prime}$, the difference in the predicted $\mathcal{P}$ is

$$
\begin{aligned}
\mathcal{P}(\mathcal{O})-\mathcal{P}\left(\mathcal{O}^{\prime}\right)= & \frac{1}{\Lambda^{2}}\left[-A_{n} F_{n}^{(2)}+A_{n}^{\prime} F_{n}^{(2) \prime}\right]+\frac{1}{\Lambda^{4}}\left[-A_{n} F_{n}^{(4)}+A_{n}^{\prime} F_{n}^{(4) \prime}+\right. \\
& \left.-A_{n}^{(2)} F_{n}^{(2)}+A_{n}^{(2) \prime} F_{n}^{(2) \prime}+B_{m n} F_{m}^{(2)} F_{n}^{(2)}-B_{m n}^{\prime} F_{m}^{(2) \prime} F_{n}^{(2) \prime}\right]+\ldots
\end{aligned}
$$

which is easily evaluated via eqs. (4.13)-(4.15). This result is consistent with those in the appendix of ref. [63] and in ref. [80].

\subsection{Implementation in SMEFTsim}

SMEFTsim implements the finite renormalization procedure via replacements of the form ${ }^{11}$

$$
g_{i} \rightarrow \hat{g}_{i}+\delta g_{i},
$$

where $\hat{g}_{i}$ satisfies the SM relation $\hat{g}_{i}=K_{i}^{(0)}(\mathcal{O})$ and $\delta g_{i}$ encodes all the dependence on the Wilson coefficients. In the FeynRules models, these replacements are operated at the Lagrangian level via the lists redefConst (applied simultaneously to the redefinitions in eq. (2.11)) and redefVev, and the hats are subsequently dropped in the notation. In this way, all the SM parameters appearing explicitly in the final $\mathcal{L}_{\text {SMEFT }}$ are hatted quantities, i.e. they are conveniently defined in the exact same way as in the SM and their numerical value is directly defined by the input observables chosen.

The shifts $\delta g$ appear explicitly in the interaction terms, and they are responsible for propagating input shifts corrections to the computed processes. By construction, the de-

\footnotetext{
${ }^{10}$ Here "double insertions" refers to any contribution quadratic in the $\mathcal{L}_{6}$ coefficients. This includes contributions from the square of a diagram with one EFT insertion, as well as from the interference between SM and EFT diagrams with two EFT vertices, or EFT diagrams with a single interaction $\propto C^{2}$. The latter generally stem from field or parameter redefinitions in the Lagrangian.

${ }^{11}$ Ref. [63] used the notation $\bar{g}_{i} \rightarrow \hat{g}_{i}+\delta g_{i}$ from ref. [52]. This is completely equivalent to the one used here, dropping the bars.
} 
pendence on $\delta g$ themselves is universal, while their expressions in terms of Wilson coefficients are fixed by the input scheme choice:

$$
\delta g_{i}=\frac{1}{\Lambda^{2}} K_{i}^{(2)}(\mathcal{O})=-\frac{1}{\Lambda^{2}}\left(J^{-1}\right)_{i n} F_{n}^{(2)} .
$$

As noted in section 2, because we work at order $\Lambda^{-2}$, the replacements of eq. (4.19) only need to be performed on $\mathcal{L}_{\mathrm{SM}}$ and only linear terms in $\delta g$ need to be retained. Moreover, one can replace $v_{T} \rightarrow \hat{v}$ in the $\bar{C}_{\alpha}$ notation, eq. (2.4).

This procedure is implemented for parameters listed in the Higgs, EW and Yukawa sectors in (4.3), as described below. Eq. (4.4) makes manifest that the extraction of SM parameters from global fits can become problematic when generalized to the SMEFT. Whenever this set of equations is not invertible, it is not possible to find a simple form for $g_{i}$ that expands around the SM solution. A consistent treatment of EFT corrections to such input observables would require to extract simultaneously $g_{i}$ and $C_{\alpha}$, which can be very unpractical or even unfeasible, in the presence of blind directions.

In the case of the CKM parameters, this issue has been overcome in ref. [81], where an optimal set of 4 input measurements was proposed, that allows for a treatment of the CKM angles and phase analogous to that of EW parameters. Its implementation is left for future versions of SMEFTsim.

The case of $\alpha_{s}$ poses a bigger challenge. The strong coupling constant can be determined from a particularly vast range of processes [82], and its extraction is often correlated to that of other physical quantities, such as parton distribution functions (PDFs). A proofof-concept analysis of SMEFT effects on the PDFs determination was presented in ref. [83], that explored the consequences of including four-fermion operators in a fit to deep-inelastic scattering data. Further studies are needed in order to define an optimal strategy for the treatment of SMEFT contributions in this context. For the time being, input shift corrections associated to the determination of $\alpha_{s}$ are omitted in SMEFTsim.

\subsection{Higgs and EW sectors}

The electroweak sector of the SM contains 4 independent quantities, that can be chosen as $g=\left\{g_{1}, g_{W}, v, \lambda\right\}$. The 4 (pseudo-)observables needed to fix their values are usually taken in the set

$$
\left\{\alpha_{\mathrm{em}}, G_{F}, m_{Z}, m_{W}, m_{h}\right\} .
$$

While $m_{h}$ always needs to be retained in order to fix $\lambda$, the choice of the 3 remaining inputs is free, and several combinations have been adopted in the literature. SMEFTsim implements the two alternative schemes $\left\{\alpha_{\mathrm{em}}, m_{Z}, G_{F}\right\}$ and $\left\{m_{W}, m_{Z}, G_{F}\right\}$, providing independent UFO models for both.

The fine structure constant $\alpha_{\mathrm{em}}(0)$ is taken to be measured in Thomson scattering, ${ }^{12}$ the Fermi constant $G_{F}$ measured in muon decays $\mu^{-} \rightarrow e^{-} \nu_{\mu} \bar{\nu}_{e}$, and $m_{W}, m_{Z}, m_{h}$ are

\footnotetext{
${ }^{12}$ As we work at tree level, only direct SMEFT corrections to Thomson scattering (i.e. to the determination of $\left.\alpha_{\mathrm{em}}(0)\right)$ are included here. The determination of $\alpha_{\mathrm{em}}\left(m_{Z}\right)$ at one loop in the SMEFT is another major open problem, as potential EFT contributions in the running have not been estimated to date. The main challenge in this task is posed by non-perturbative effects, particularly those arising as $\alpha_{\mathrm{em}}$ runs through the hadronic resonances region.
} 


\begin{tabular}{|l|l|l|}
\hline$\Delta \kappa_{H}=\bar{C}_{H \square}-\frac{\bar{C}_{H D}}{4}$ & $\bar{C}_{H D}+\frac{2 g_{1} g_{W}}{g_{1}^{2}+g_{W}^{2}} \bar{C}_{H W B}$ \\
$\Delta m_{Z}^{2}=\frac{\bar{C}_{\mathrm{e}}}{2}=-\frac{2 g_{1} g_{W}}{g_{1}^{2}+g_{W}^{2}} \bar{C}_{H W B}$ & $\begin{array}{ll}\text { general } \\
\text { U35, MFV, } \\
\text { topU31 }\end{array}$ & $\left.\Delta G_{F}=2 \bar{C}_{H l}^{(3)}-\bar{C}_{l l}^{\prime(3)}\right)_{11}+\left(\bar{C}_{H l}^{(3)}\right)_{22}-\left(\bar{C}_{l l}\right)_{1221}$ \\
$\Delta m_{h}^{2}=2 \Delta \kappa_{H}-\frac{3}{2 \lambda} \bar{C}_{H}$ & top & $\Delta G_{F}=\bar{C}_{H l, 11}^{(3)}+\bar{C}_{H l, 22}^{(3)}-\frac{\bar{C}_{l l, 1221}}{2}$ \\
\hline
\end{tabular}

Table 4. Expressions of input parameter shifts and the kinetic correction $\Delta \kappa_{H}$ (defined in (2.6)) in terms of Wilson coefficients. The left column is common to all flavor versions, while $\Delta G_{F}$ varies as indicated in the right column. We use the notation $\bar{C}_{\alpha}=C_{\alpha}\left(\hat{v}^{2} / \Lambda^{2}\right)$.

defined as the bosons' pole masses, see ref. [1] and references therein for further details. With these definitions, at tree level: ${ }^{13}$

$$
\begin{array}{rlrl}
\alpha_{\mathrm{em}} & =\frac{1}{4 \pi} \frac{g_{W}^{2} g_{1}^{2}}{g_{W}^{2}+g_{1}^{2}}\left[1+\Delta \alpha_{\mathrm{em}}\right], & G_{F} & =\frac{1}{\sqrt{2} v_{T}^{2}}\left[1+\Delta G_{F}\right], \\
m_{W}^{2} & =\frac{v_{T}^{2}}{4} g_{W}^{2}, & m_{Z}^{2}=\frac{v_{T}^{2}}{4}\left(g_{W}^{2}+g_{1}^{2}\right)\left[1+\Delta m_{Z}^{2}\right], \\
m_{h}^{2}=2 v_{T}^{2} \lambda\left[1+\Delta m_{h}^{2}\right] . &
\end{array}
$$

The $\Delta$ quantities are dimensionless and defined in table 4: $\Delta G_{F}$ is inferred computing the muon decay width at tree level in the SMEFT, while the remaining shifts can be read from the relevant Lagrangian terms. In particular, the contributions in $\bar{C}_{H W B}$ to $\Delta \alpha_{\mathrm{em}}, \Delta m_{Z}^{2}$ follow directly from eq. (2.18) and $\Delta m_{h}^{2}$ follows from eq. (2.8).

The relations (4.21)-(4.23) can be directly mapped to the notation of eq. (4.4): for instance

$$
F_{G_{F}}^{(0)}=\frac{1}{\sqrt{2} v_{T}^{2}}, \quad \frac{1}{\Lambda^{2}} F_{G_{F}}^{(2)}=F_{G_{F}}^{(0)} \Delta G_{F},
$$

and analogously for the other observables.

\subsection{1 $\left\{\alpha_{\mathrm{em}}, m_{Z}, G_{F}\right\}$ scheme}

Solving 3 of the 4 eqs. in (4.21), (4.22), plus eq. (4.23), gives expressions for the SM parameters of the form of (4.5). Let us choose the input quantities $\mathcal{O}^{(\alpha)}=\left\{\alpha_{\mathrm{em}}, m_{Z}^{2}, G_{F}, m_{h}^{2}\right\}$.

It is convenient to define the vector of SM parameters as $g=\left\{g_{1}^{2}, g_{W}^{2}, v_{T}^{2}, \lambda\right\}$. The SM solutions $\hat{g}_{i} \equiv K_{i}^{(0)}(\mathcal{O})$ are then

$$
\hat{g}_{1}^{2}=\frac{4 \pi \alpha_{\mathrm{em}}}{c_{\hat{\theta}}^{2}}, \quad \hat{g}_{W}^{2}=\frac{4 \pi \alpha_{\mathrm{em}}}{s_{\hat{\theta}}^{2}}, \quad \hat{v}^{2}=\frac{1}{\sqrt{2} G_{F}}, \quad \hat{\lambda}=\frac{m_{h}^{2} G_{F}}{\sqrt{2}},
$$

\footnotetext{
${ }^{13}$ The normalization of $\Delta G_{F}$ has been modified compared to previous SMEFTsim versions in order to homogenize the notation with the remaining shifts.
} 
having defined the weak angle $\hat{\theta}$ as

$$
s_{\hat{\theta}}^{2}=\sin ^{2} \hat{\theta} \equiv \frac{\hat{g}_{1}^{2}}{\hat{g}_{1}^{2}+\hat{g}_{W}^{2}}=\frac{1}{2}\left[1-\sqrt{1-\frac{2 \sqrt{2} \pi \alpha_{\mathrm{em}}}{G_{F} m_{Z}^{2}}}\right] .
$$

The Jacobian $J=\partial \mathcal{O}^{(\alpha)} / \partial g$ defined in eq. (4.10) takes the form

$$
J=\frac{1}{4}\left(\begin{array}{cccc}
c_{\hat{\theta}}^{4} / \pi & s_{\hat{\theta}}^{4} / \pi & & \\
\hat{v}^{2} & \hat{v}^{2} & \hat{g}_{W}^{2} / c_{\hat{\theta}}^{2} & \\
& & -2 \sqrt{2} / \hat{v}^{4} & \\
& & 8 \hat{\lambda} & 8 \hat{v}^{2}
\end{array}\right) .
$$

Taking the inverse and plugging it in eq. (4.7), one obtains explicit expressions for the parameter shifts defined as in (4.20):

$$
\begin{aligned}
g_{1}^{2} & =\hat{g}_{1}^{2}\left[1+2 \frac{\delta g_{1}}{\hat{g}_{1}}\right], & \frac{\delta g_{1}}{\hat{g}_{1}} & =\frac{s_{\hat{\theta}}^{2}}{2 c_{2 \hat{\theta}}}\left(\Delta m_{Z}^{2}+\Delta G_{F}\right)-\frac{c_{\hat{\theta}}^{2}}{2 c_{2 \hat{\theta}}} \Delta \alpha_{\mathrm{em}}, \\
g_{W}^{2} & =\hat{g}_{W}^{2}\left[1+2 \frac{\delta g_{W}}{\hat{g}_{W}}\right], & \frac{\delta g_{W}}{\hat{g}_{W}} & =-\frac{c_{\hat{\theta}}^{2}}{2 c_{2 \hat{\theta}}}\left(\Delta m_{Z}^{2}+\Delta G_{F}\right)+\frac{s_{\hat{\theta}}^{2}}{2 c_{2 \hat{\theta}}} \Delta \alpha_{\mathrm{em}}, \\
v_{T}^{2} & =\hat{v}^{2}\left[1+2 \frac{\delta v}{\hat{v}}\right], & \frac{\delta v}{\hat{v}} & =\frac{\Delta G_{F}}{2}, \\
\lambda & =\hat{\lambda}\left[1+\frac{\delta \lambda}{\hat{\lambda}}\right], & \frac{\delta \lambda}{\hat{\lambda}} & =-\Delta G_{F}-\Delta m_{h}^{2} .
\end{aligned}
$$

It can be convenient, as a shorthand notation, to define a shift for $\sin ^{2} \theta$. In the input schemes considered here, this is always a predicted quantity, that can be expressed as

$$
\delta s_{\theta}^{2}=2 c_{\hat{\theta}}^{2} s_{\hat{\theta}}^{2}\left(\frac{\delta g_{1}}{\hat{g}_{1}}-\frac{\delta g_{W}}{\hat{g}_{W}}\right)+\Delta s_{\theta}^{2},
$$

with $\Delta s_{\theta}^{2}$ defined in eq. (2.24).

With this input scheme choice, $m_{W}$ is also a predicted quantity and its expression can be derived from eq. (4.12). From eq. (4.22), we have that $P_{m_{W}^{2}}^{(2)}=0$, so

$$
\begin{aligned}
m_{W}^{2} & =P_{m_{W}^{2}}^{(0)}-\frac{1}{\Lambda^{2}} \frac{\partial P_{m_{W}^{2}}^{(0)}}{\partial g_{i}}\left(J^{-1}\right)_{i n} F_{n}^{(2)} \\
& =\frac{\hat{v}^{2} \hat{g}_{W}^{2}}{4}\left[1-\frac{c_{\hat{\theta}}^{2}}{c_{2 \hat{\theta}}} \Delta m_{Z}^{2}+\frac{s_{\hat{\theta}}^{2}}{c_{2 \hat{\theta}}}\left(\Delta \alpha_{\mathrm{em}}-\Delta G_{F}\right)\right] \\
& =\frac{\hat{v}^{2} \hat{g}_{W}^{2}}{4}\left[1+2 \frac{\delta v}{\hat{v}}+2 \frac{\delta g_{W}}{\hat{g}_{W}}\right] \\
& =\hat{m}_{W}^{2}\left[1+2 \frac{\delta m_{W}}{\hat{m}_{W}}\right],
\end{aligned}
$$

where we defined the shift

$$
\begin{aligned}
\frac{\delta m_{W}}{\hat{m}_{W}} & =\frac{\delta v}{\hat{v}}+\frac{\delta g_{W}}{\hat{g}_{W}}= \\
& =-\frac{s_{2 \hat{\theta}}}{4 c_{2 \hat{\theta}}}\left[\frac{1}{2} \frac{c_{\hat{\theta}}}{s_{\hat{\theta}}} \bar{C}_{H D}+2 \bar{C}_{H W B}+\frac{s_{\hat{\theta}}}{c_{\hat{\theta}}}\left(\left(\bar{C}_{H l}^{(3)}\right)_{11}+\left(\bar{C}_{H l}^{(3)}\right)_{22}-\left(\bar{C}_{l l}\right)_{1221}\right)\right] .
\end{aligned}
$$


The second line was evaluated with generic flavor indices for $\Delta G_{F}$, and it can be easily mapped to other flavor structures with the dictionary in appendix C. Finally, it is worth noting that electromagnetic interactions do not receive any corrections in this scheme:

$$
\frac{\delta e}{\hat{e}}=c_{\hat{\theta}}^{2} \frac{\delta g_{1}}{\hat{g}_{1}}+s_{\hat{\theta}}^{2} \frac{\delta g_{W}}{\hat{g}_{W}}+\frac{1}{2} \Delta \alpha_{\mathrm{em}}=0,
$$

consistent with $\alpha_{\mathrm{em}}$ being an input quantity.

\subsection{2 $\left\{m_{W}, m_{Z}, G_{F}\right\}$ scheme}

Choosing the input observables $\mathcal{O}^{\left(m_{W}\right)}=\left\{m_{W}^{2}, m_{Z}^{2}, G_{F}, m_{h}^{2}\right\}$, the SM expressions $\hat{g}_{i} \equiv$ $K_{i}^{(0)}(\mathcal{O})$ for the relevant parameters are

$$
\hat{g}_{1}^{2}=4 \sqrt{2} G_{F} m_{Z}^{2} s_{\hat{\theta}}^{2}, \quad \hat{g}_{W}^{2}=4 \sqrt{2} G_{F} m_{W}^{2}, \quad \hat{v}^{2}=\frac{1}{\sqrt{2} G_{F}}, \quad \hat{\lambda}=\frac{m_{h}^{2} G_{F}}{\sqrt{2}},
$$

with the weak angle defined by

$$
s_{\hat{\theta}}^{2} \equiv \frac{\hat{g}_{1}^{2}}{\hat{g}_{1}^{2}+\hat{g}_{W}^{2}}=1-\frac{m_{W}^{2}}{m_{Z}^{2}} .
$$

The Jacobian $J=\partial \mathcal{O}^{\left(m_{W}\right)} / \partial g$ takes the form

$$
J=\frac{1}{4}\left(\begin{array}{cccc} 
& \hat{v}^{2} & \hat{g}_{W}^{2} & \\
\hat{v}^{2} & \hat{v}^{2} & \hat{g}_{W}^{2} / c_{\hat{\theta}}^{2} & \\
& & -2 \sqrt{2} / \hat{v}^{4} & \\
& & 8 \hat{\lambda} & 8 \hat{v}^{2}
\end{array}\right),
$$

and from eq. (4.7) one has

$$
\begin{aligned}
g_{1}^{2} & =\hat{g}_{1}^{2}\left[1+2 \frac{\delta g_{1}}{\hat{g}_{1}}\right], & \frac{\delta g_{1}}{\hat{g}_{1}} & =-\frac{1}{2}\left[\Delta G_{F}+\frac{\Delta m_{Z}^{2}}{s_{\hat{\theta}}^{2}}\right], \\
g_{W}^{2} & =\hat{g}_{W}^{2}\left[1+2 \frac{\delta g_{W}}{\hat{g}_{W}}\right], & \frac{\delta g_{W}}{\hat{g}_{W}} & =-\frac{\Delta G_{F}}{2}, \\
v_{T}^{2} & =\hat{v}^{2}\left[1+2 \frac{\delta v}{\hat{v}}\right], & \frac{\delta v}{\hat{v}} & =\frac{\Delta G_{F}}{2}, \\
\lambda & =\hat{\lambda}\left[1-\frac{\delta \lambda}{\hat{\lambda}}\right], & \frac{\delta \lambda}{\hat{\lambda}} & =-\Delta G_{F}-\Delta m_{h}^{2} .
\end{aligned}
$$

With this input scheme choice, $\alpha_{\mathrm{em}}$ is now a predicted quantity. From eq. (4.12):

$$
\begin{aligned}
\alpha_{\mathrm{em}} & =P_{\alpha_{\mathrm{em}}}^{(0)}+\frac{1}{\Lambda^{2}}\left[\frac{\partial P_{\alpha_{\mathrm{em}}}^{(0)}}{\partial g_{i}}\left(J^{-1}\right)_{i n} F_{n}^{(2)}+P_{\alpha_{\mathrm{em}}}^{(2)}\right] \\
& =\frac{1}{4 \pi} \frac{\hat{g}_{1}^{2} \hat{g}_{W}^{2}}{\hat{g}_{1}^{2}+\hat{g}_{W}^{2}}\left[1-\Delta G_{F}-\frac{c_{\hat{\hat{\theta}}}^{2}}{s_{\hat{\theta}}^{2}} \Delta m_{Z}^{2}+\Delta \alpha_{\mathrm{em}}\right] \\
& =\frac{1}{4 \pi} \frac{\hat{g}_{1}^{2} \hat{g}_{W}^{2}}{\hat{g}_{1}^{2}+\hat{g}_{W}^{2}}\left[1+2 c_{\hat{\theta}}^{2} \frac{\delta g_{1}}{\hat{g}_{1}}+2 s_{\hat{\theta}}^{2} \frac{\delta g_{W}}{\hat{g}_{W}}+\Delta \alpha_{\mathrm{em}}\right] \\
& =\frac{\hat{e}^{2}}{4 \pi}\left[1+2 \frac{\delta e}{\hat{e}}\right] .
\end{aligned}
$$




\begin{tabular}{|c|c|c|c|c|c|}
\hline & $\Delta M_{l}$ & \multicolumn{2}{|l|}{$\Delta M_{u}$} & \multicolumn{2}{|l|}{$\Delta M_{d}$} \\
\hline general & $\frac{1}{2} \bar{C}_{e H}^{\dagger}$ & \multicolumn{2}{|l|}{$\frac{1}{2} \bar{C}_{u H}^{\dagger}$} & \multicolumn{2}{|l|}{$\frac{1}{2} \bar{C}_{d H}^{\dagger} V$} \\
\hline U35 & $\frac{1}{2} \bar{C}_{e H}^{*} Y_{l}^{(d)}$ & \multicolumn{2}{|l|}{$\frac{1}{2} \bar{C}_{u H}^{*} Y_{u}^{(d)}$} & \multicolumn{2}{|l|}{$\frac{1}{2} \bar{C}_{d H}^{*} Y_{d}^{(d)}$} \\
\hline MFV & $\frac{1}{2} \bar{C}_{e H} Y_{l}^{(d)}$ & \multicolumn{2}{|c|}{$\frac{1}{2} Y_{u}^{(d)}\left[\bar{C}_{u H}^{(0)}+\left(\Delta^{u} \bar{C}_{u H}\right) S^{q u}+\left(\Delta^{d} \bar{C}_{u H}\right) S^{q d}\right]$} & \multicolumn{2}{|c|}{$\frac{1}{2} Y_{d}^{(d)} V^{\dagger}\left[\bar{C}_{d H}^{(0)}+\left(\Delta^{u} \bar{C}_{d H}\right) S^{q u}+\left(\Delta^{d} \bar{C}_{d H}\right) S^{q d}\right] V$} \\
\hline \multirow[t]{2}{*}{ top } & $\frac{1}{2}\left(\bar{C}_{e H}\right)_{p p}^{*}$ & $\frac{1}{2} \bar{C}_{u H}^{*} Y_{u}^{(d)}$ & $(u, c)$ & $\frac{1}{2} \bar{C}_{d H}^{*} Y_{d}^{(d)}$ & $(d, s)$ \\
\hline & & $\frac{1}{2} \bar{C}_{t H}^{*}$ & $(t)$ & $\frac{1}{2} \bar{C}_{b H}^{*}$ & (b) \\
\hline \multirow[t]{2}{*}{ topU31 } & $\frac{1}{2} \bar{C}_{e H}^{*} Y_{l}^{(d)}$ & $\frac{1}{2} \bar{C}_{u H}^{*} Y_{u}^{(d)}$ & $(u, c)$ & $\frac{1}{2} \bar{C}_{d H}^{*} Y_{d}^{(d)}$ & $(d, s)$ \\
\hline & & $\frac{1}{2} \bar{C}_{t H}^{*}$ & $(t)$ & $\frac{1}{2} \bar{C}_{b H}^{*}$ & (b) \\
\hline
\end{tabular}

Table 5. SMEFT corrections to the fermion mass matrices for each flavor assumption, see section 3 for all definitions. All Wilson coefficients are scalar quantities, except in the general case, where they are $3 \times 3$ matrices. In the MFV case all parameters are real. In the top and topU3I cases $\Delta M_{u, d}, Y_{u, d}$ are $2 \times 2$ matrices for the first two generations, and the mass term for the $t, b$ quarks are independent. We use the notation $\bar{C}_{\alpha}=C_{\alpha}\left(\hat{v}^{2} / \Lambda^{2}\right)$ and the results are given in the mass basis of the up-quarks and charged leptons.

It can be instructive to write the final form of the Higgs potential, once the input shifts are applied onto eq. (2.8). For both input schemes considered here, the result is

$$
\begin{aligned}
V(H)+\mathcal{L}_{6}= & h^{2} \hat{\lambda} \hat{v}^{2}+h^{3} \hat{\lambda} \hat{v}\left[1-\frac{\Delta G_{F}}{2}+\Delta \kappa_{H}-\frac{1}{\hat{\lambda}} \bar{C}_{H}\right] \\
& +h^{4} \frac{\hat{\lambda}}{4}\left[1-\Delta G_{F}+2 \Delta \kappa_{H}-\frac{6}{\hat{\lambda}} \bar{C}_{H}\right]-\frac{3}{4} \frac{h^{5}}{\hat{v}} \bar{C}_{H}-\frac{1}{8} \frac{h^{6}}{\hat{v}^{2}} \bar{C}_{H} .
\end{aligned}
$$

\subsection{Yukawa sector}

To fix the SM Yukawa couplings, we take fermion masses as input quantities. From the propagators' poles, at tree level, we have

$$
M_{\psi}=\frac{v_{T}}{\sqrt{2}}\left[Y_{\psi}^{(d)}-\Delta M_{\psi}\right]
$$

with $Y_{\psi}^{(d)}$ diagonal. In the top, topU3l cases the index $\psi$ runs over $\psi=\{l, u, d, t, b\}$ so that $M_{l}, Y_{l}, \Delta M_{l}$ are $3 \times 3$ tensors, $M_{u, d}, Y_{u, d}, \Delta M_{u, d}$ are $2 \times 2$ and $M_{t, b}, Y_{t, b}, \Delta M_{t, b}$ are scalar quantities. In the other flavor setups $\psi=\{l, u, d\}$ and all quantities are $3 \times 3$ matrices. The SMEFT corrections $\Delta M_{\psi}$ are given in table 5 for each flavor assumption. The SM solutions are

$$
\hat{Y}_{\psi}^{(d)}=\frac{\sqrt{2}}{\hat{v}} M_{\psi},
$$

and the shifts $\delta Y_{\psi}$ have the form

$$
Y_{\psi}^{(d)} \rightarrow \hat{Y}_{\psi}^{(d)}+\delta Y_{\psi}^{(d)}, \quad \delta Y_{\psi}^{(d)}=-\frac{\Delta G_{F}}{2} \hat{Y}_{\psi}^{(d)}+\Delta M_{\psi},
$$

where $\Delta G_{F}$ enters via eq. (4.41) and $\Delta M_{\psi}$ is non-diagonal and non-Hermitian in general. The expressions (4.46), (4.47) can be easily generalized to setups where $M_{\psi}$ is not diagonal, by applying the appropriate flavor rotations to both sides of the equations. 
The net effect of the finite renormalization procedure is that $\Delta M_{\psi}$ corrections to the fermion mass terms are recast into corrections to the $h \bar{\psi} \psi$ couplings. In unitary gauge, the Lagrangian resulting from the replacements $(2.7),(4.47)$ is

$$
\begin{aligned}
\mathcal{L}_{\text {Yukawa }}+\mathcal{L}_{6}^{(5)}= & -\frac{\bar{d}_{R, p} d_{L, r}}{\sqrt{2}}\left[\hat{v} \hat{Y}_{d}^{(d)}+h \hat{Y}_{d}^{(d)}\left(1-\frac{\Delta G_{F}}{2}+\Delta \kappa_{H}\right)-\left(2 h+\frac{3 h^{2}}{\hat{v}}+\frac{h^{3}}{\hat{v}^{2}}\right) \Delta M_{d}\right]_{p r} \\
& -\frac{\bar{u}_{R, p} u_{L, r}}{\sqrt{2}}\left[\hat{v} \hat{Y}_{u}^{(d)}+h \hat{Y}_{u}^{(d)}\left(1-\frac{\Delta G_{F}}{2}+\Delta \kappa_{H}\right)-\left(2 h+\frac{3 h^{2}}{\hat{v}}+\frac{h^{3}}{\hat{v}^{2}}\right) \Delta M_{u}\right]_{p r} \\
& -\frac{\bar{e}_{R, p} e_{L, r}}{\sqrt{2}}\left[\hat{v} \hat{Y}_{l}^{(d)}+h \hat{Y}_{l}^{(d)}\left(1-\frac{\Delta G_{F}}{2}+\Delta \kappa_{H}\right)-\left(2 h+\frac{3 h^{2}}{\hat{v}}+\frac{h^{3}}{\hat{v}^{2}}\right) \Delta M_{l}\right]_{p r} \\
& + \text { h.c. }
\end{aligned}
$$

In the top, topU3I models analogous terms with $t, b$ quarks are also present.

In the FeynRules implementation, the common shifts $\delta v, \delta \lambda, \delta Y_{\psi}$ are automatically replaced with the corresponding expressions in terms of Wilson coefficients. On the other hand, the dependence on the EW shifts $\delta g_{1}, \delta g_{W}$ is left explicit in the Lagrangian, as it is identical for all EW input schemes. Once an inputs set is selected, these shifts can be traded for Wilson coefficients expressions: in Mathematica this is done via the replacement lists alphaShifts or MwShifts. In the UFO models all shifts are replaced with the Wilson coefficient expressions.

\section{SM loop-generated Higgs interactions}

Because SMEFTsim is designed as a tree-level model, it cannot reproduce processes that only occur at 1-loop. In fact, estimating SMEFT corrections to observables that are genuinely loop-generated both in the SM and at $d=6$ level is beyond the scope of SMEFTsim. Nevertheless, there are cases where a 1-loop SM processes receives tree $\mathcal{L}_{6}$ corrections. This notably happens in a few relevant Higgs production and decay channels.

In order to enable an estimate of interference terms between $\mathcal{L}_{6}$ and SM diagrams for the processes $g g \rightarrow h, h \rightarrow \gamma \gamma, h \rightarrow Z \gamma$, SMEFTsim implements effective SM interactions obtained in the large $m_{t}$ limit. This formally corresponds to matching the SM onto an EFT (we will refer to this as "top-EFT") where the top quark has been integrated out. The advantage of this approach is that the top loops are effectively reduced to point vertices that can be inserted in tree diagrams. The obvious caveat is that the top-EFT is only valid in a limited kinematic region, as discussed below.

SMEFTsim 3.0 contains $h \gamma \gamma, h Z \gamma, h g g, h g g g$ and $h g g g g$ interactions with contributions up to $\mathcal{O}\left(m_{t}^{-2}\right)$, i.e. $d=7$ in the top-EFT. The implemented Lagrangian is:

$$
\mathcal{L}_{\text {SMhloop }}=g_{H \gamma \gamma} \mathcal{O}_{\gamma \gamma}+g_{H Z \gamma} O_{Z \gamma}+g_{H g g}^{(1)} \mathcal{O}_{g g}^{(1)}+\frac{1}{m_{t}^{2}} \sum_{i=2}^{5} g_{H g g}^{(i)} \mathcal{O}_{g g}^{(i)},
$$

where

$$
\begin{aligned}
\mathcal{O}_{\gamma \gamma} & =A_{\mu \nu} A^{\mu \nu} \frac{h}{v}, & \mathcal{O}_{z \gamma} & =Z_{\mu \nu} A^{\mu \nu} \frac{h}{v}, \\
\mathcal{O}_{g g}^{(2)} & =D_{\sigma} G_{\mu \nu}^{a} D^{\sigma} G^{a \mu \nu} \frac{h}{v}, & \mathcal{O}_{g g}^{(3)} & =f_{a b c} G_{\mu}^{a \nu} G_{\nu}^{b \sigma} G_{\sigma}^{c \mu} \frac{h}{v}, \\
\mathcal{O}_{g g}^{(4)} & =D^{\mu} G_{\mu \nu}^{a} D_{\sigma} G^{a \sigma \nu} \frac{h}{v}, & \mathcal{O}_{g g}^{(5)} & =G^{a \mu \nu} D_{\nu} D^{\sigma} G_{\sigma \mu}^{a} \frac{h}{v} .
\end{aligned}
$$


The corresponding $g$ coefficients are fixed via a 1-loop matching procedure of the SM onto the top-EFT. For the $h \gamma \gamma$ and $h Z \gamma$ interactions we use the results from refs. [84-87], that include loops of both top quarks and $W$ bosons:

$$
\begin{aligned}
g_{H \gamma \gamma} & =\frac{e^{2}}{8 \pi^{2}}\left[I_{w}\left(\frac{m_{h}^{2}}{4 m_{W}^{2}}\right)+\frac{4}{3} I_{f}\left(\frac{m_{h}^{2}}{4 m_{t}^{2}}, 0\right)\right], \\
g_{H Z \gamma} & =\frac{e^{2}}{4 \pi^{2}}\left[t_{\theta} I_{w}^{Z}\left(\frac{m_{h}^{2}}{4 m_{W}^{2}}, \frac{m_{Z}^{2}}{4 m_{W}^{2}}\right)+\left(\frac{1}{2}-\frac{4}{3} s_{\theta}^{2}\right) I_{f}\left(\frac{m_{h}^{2}}{4 m_{t}^{2}}, \frac{m_{Z}^{2}}{4 m_{t}^{2}}\right)\right] .
\end{aligned}
$$

The loop functions $I_{f}, I_{w}, I_{w}^{Z}$ are evaluated in the limit where the Higgs boson is on-shell and higher order corrections are simply obtained via Taylor-expansion, retaining terms up to $\mathcal{O}\left(m_{t}^{-2} m_{W}^{-6}\right)$ :

$$
\begin{aligned}
I_{f}(a, b)= & \int_{0}^{1} \int_{0}^{1-x} \frac{1-4 x y}{1-4(a-b) x y-4 b y(1-y)} d y d x \\
= & \frac{1}{3}+\frac{7 a}{90}+\frac{11 b}{90}+\mathcal{O}\left(a^{2}, b^{2}\right) \\
I_{w}(a)= & \int_{0}^{1} \int_{0}^{1-x} \frac{-4+6 x y+4 a x y}{1-4 a x y} d y d x \\
= & -\frac{7}{4}-\frac{11 a}{30}-\frac{19 a^{2}}{105}-\frac{58 a^{3}}{525}+\mathcal{O}\left(a^{4}\right) \\
I_{w}^{Z}(a, b)= & \frac{1}{t_{\theta}^{2}} \int_{0}^{1} \int_{0}^{1-x} \frac{\left(5-t_{\theta}^{2}+2 a\left(1-t_{\theta}^{2}\right)\right) x y+t_{\theta}^{2}-3}{1-4(a-b) x y-4 b y(1-y)} d y d x \\
= & 1+\frac{5-t_{\theta}^{2}}{12} a-b+\frac{t_{\theta}^{2}-9}{9} a b+\frac{2\left(5-t_{\theta}^{2}\right)}{45} a^{2}+\frac{56 b^{2}}{45}+\frac{5-t_{\theta}^{2}}{35} a^{3}-\frac{12 b^{3}}{7}+ \\
& -\frac{2\left(7-t_{\theta}^{2}\right)}{15} a^{2} b+\frac{17\left(13-t_{\theta}^{2}\right)}{105} a b^{2}+\mathcal{O}\left(a^{3}, b^{3}\right) .
\end{aligned}
$$

For the Higgs-gluon interactions, the matching has been performed in refs. [88-90] up to dimension 7 in the top expansion: ${ }^{14}$

$$
\begin{aligned}
& g_{H g g}^{(1)}=\frac{g_{s}^{2}}{48 \pi^{2}}+\mathcal{O}\left(g_{s}^{4}\right), \\
& g_{H g g}^{(2)}=-\frac{7 g_{s}^{2}}{2880 \pi^{2}}+\mathcal{O}\left(g_{s}^{4}\right), \\
& g_{H g g}^{(3)}=\frac{g_{s}^{3}}{240 \pi^{2}}+\mathcal{O}\left(g_{s}^{5}\right), \\
& g_{H g g}^{(4)}=\frac{g_{s}^{2}}{1440 \pi^{2}}+\mathcal{O}\left(g_{s}^{4}\right), \\
& g_{H g g}^{(5)}=\frac{g_{s}^{2}}{80 \pi^{2}}+\mathcal{O}\left(g_{s}^{4}\right) .
\end{aligned}
$$

\footnotetext{
${ }^{14}$ There is a sign difference in the definition of $\mathcal{O}_{3}$ compared to refs. [88, 89]. The sign of $C_{3}$ is also affected by the sign in the covariant derivative definition, that was taken with the opposite convention in ref. [90].
} 
Note that the $d=7$ operators produce interactions with one Higgs and up to 6 gluon legs. While the full gauge-invariant Lagrangian is implemented in the FeynRules models, only vertices with up to 4 gluon legs ( $h g g, h g g g, h g g g g)$ were exported to the UFOs. The Feynman rules of the hggggg and hgggggg vertices are extremely complex both in the color and Lorentz structures, to the point that their inclusion makes the Monte Carlo event generation computationally challenging. They are available upon request.

\subsection{Validity of the approximations used}

The Higgs interactions described in this section are implemented to the specific purpose of enabling the simulation of Higgs production and decay processes. In general, these vertices should not be inserted into other arbitrary processes. In MadGraph5_aMC@NLO, the insertions can be controlled at the diagram generation level via the interaction order SMHLOOP $=1$ that is assigned to all the $g$ couplings in the Lagrangian (5.1), see also section 8.2.

The following limitations should also be kept in mind:

- The implementation relies on the top-EFT formalism, that is only valid when the momentum $q$ flowing through the effective vertex is $q<m_{t}$. This condition is always fulfilled for $g g \rightarrow h$ with no extra jets, for which the top-EFT reproduces the 1loop SM cross-section within an accuracy of a few permille. With more complex final states, a validity threshold is present and it can translate differently in terms of measured observables, depending on the process.

Figure 1 shows the relative deviation of the top-EFT predictions from the 1-loop SM results for $d \sigma / d p_{T}(h)$ in $p p \rightarrow h j$ and $p p \rightarrow h j j$, as obtained at parton level with MadGraph5_aMC@NLO, in a 4-flavor scheme and neglecting all electroweak contributions. The SM prediction was obtained generating 100000 events for each $p p \rightarrow h j$ channel and for $q q \rightarrow h q q$, and 50000 events for the remaining $p p \rightarrow h j j$ channels, with the loop_sm UFO. The associated PDF and scale uncertainties were estimated with the MadGraph5_aMC@NLO functionalities [91], and their combination in quadrature is shown for reference as a grey band. The top-EFT predictions were obtained reweighting the events with SMEFTsim. The lines in color compare three different implementations of the top-EFT: including all operators up to $d=7$ (red), including only the $d=5$ operator $\mathcal{O}_{g g}^{(1)}$ (blue) and including only the $g g h$ vertex as in the previous SMEFTsim versions (orange), see the next subsection. The statistical uncertainty associated to each line is shown as a colored band surrounding the solid curves. Because the statistical errors associated to the reweighted histogram and to the original one are fully correlated, in most cases, the uncertainty on their ratio cancels and it is not visible on the plot. Uncertainties due to the reweighting procedure itself have been neglected, in the absence of a prescription for their estimation.

For both $p p \rightarrow h j$ and $p p \rightarrow h j j$, the total cross section is dominated by $g g$ - and $q g$-initiated channel, for which the $m_{t} \rightarrow \infty$ approximation breaks down roughly at $p_{T}(h) \simeq 250 \mathrm{GeV}[89,90,92-95]$. Within the top-EFT validity regime, the $d=7$ implementation reproduces the SM 1-loop result within an accuracy of few \%, see also ref. [90]. The large $m_{t}$ approximation fails most significantly in $q q$-initiated 

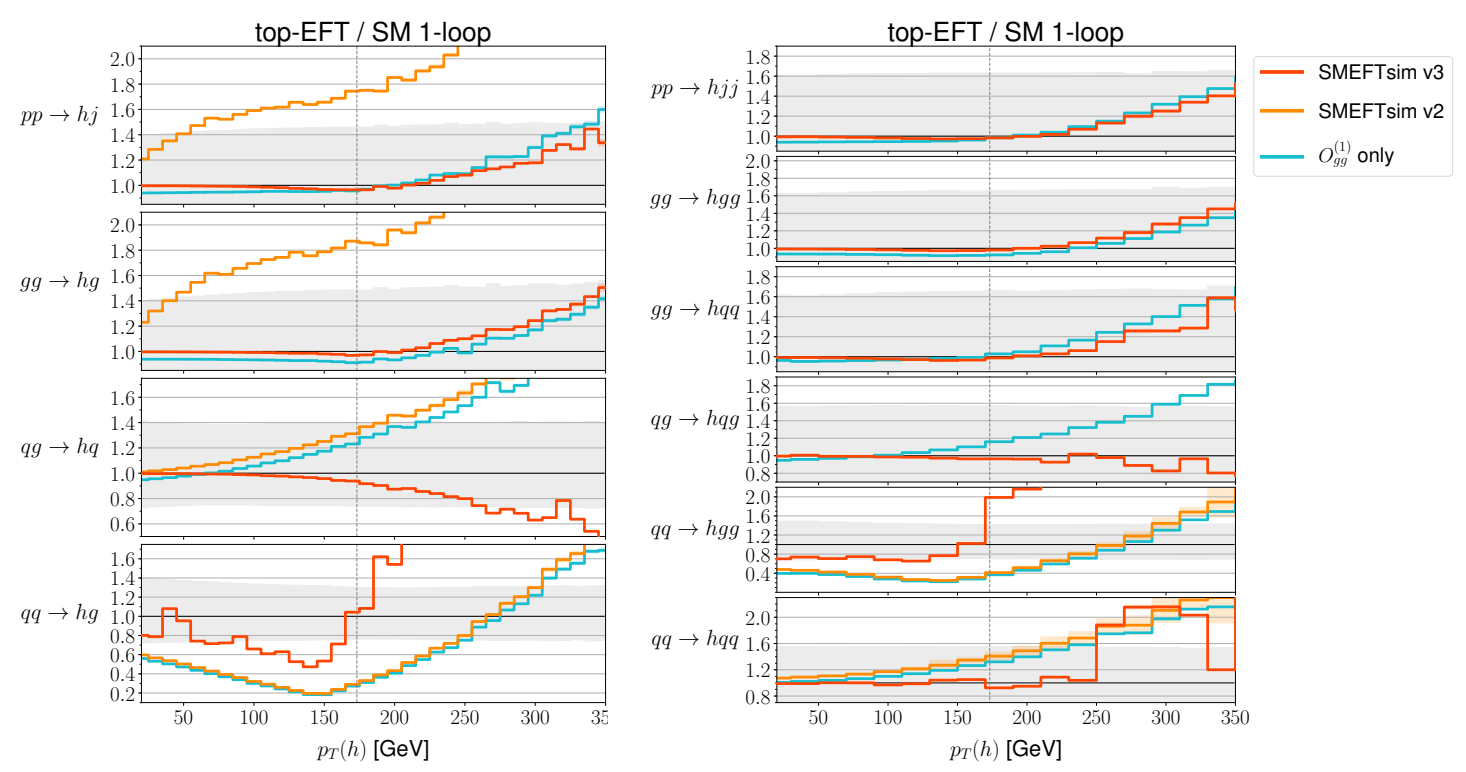

Figure 1. Top-EFT predictions for $d \sigma / d p_{T}(h)$ in $p p \rightarrow h j$ (left) and $p p \rightarrow h j j$ (right), normalized to the SM 1-loop results, see text for the calculation details. In both figures, the first panel shows the combined result, while the lower ones give the breakdown into the contributing channels. Note that here $q$ generically denotes a light quark or antiquark. The statistical uncertainties are plotted in color, and they are not visible in most cases. For reference, the gray bands show the systematic uncertainty on the SM event generation and the vertical dashed line marks $p_{T}=m_{t}$, where the top-EFT is expected to break down. SMEFTsim v2 curves for $p p \rightarrow h j j, g g \rightarrow h g g, g g \rightarrow h q q$, $q g \rightarrow h q g$ lie above the plotted range.

processes that, nevertheless, give a negligible contribution to the total cross section. This behavior is due to the quarks' PDFs preferring significantly larger $x$ compared to the gluon one, which leads to large $\hat{s}$ contributions being suppressed for $g g$ and $q g$ initial states, but not for $q q[94]$.

- The operators $O_{H g g}^{(i)}$ form a complete basis up to $\mathcal{O}\left(m_{t}^{-2}\right)$ for Higgs interactions with up to 4 gluons $[88,96]$. This means that, within the regime of validity of the topEFT, SMEFTsim can reproduce 1-loop SM Higgs production in gluon fusion with up to 2 jets.

Processes $g g \rightarrow h+n j$ with $n \geq 3$ cannot be fully reproduced with SMEFTsim, even with the inclusion of hggggg, hgggggg vertices, because a complete matching to $\mathcal{O}\left(m_{t}^{-2}\right)$ onto these vertices would require $d=9$ top-EFT operators.

- In addition to the validity of the large $m_{t}$ approximation, the implementation of $h \gamma \gamma, h Z \gamma$ assumes an on-shell Higgs in the parameterization of the loop function.

\subsection{Comparison to previous versions of SMEFTsim}

Previous SMEFTsim versions only implemented the $h g g, h \gamma \gamma$ and $h Z \gamma$ vertices, while interactions with higher numbers of gluons were omitted. In version 3.0, all the vertices induced 
by the operator $\mathcal{O}_{H g g}^{(1)}$ and vertices with up to 5 legs (4 gluons) from $\mathcal{O}_{H g g}^{(2,3,4,5)}$ are included.

Moreover, the $h g g$ interaction was previously parameterized in the on-shell Higgs limit, analogously to $h \gamma \gamma, h Z \gamma$, via a coupling [86, 87, 97]

$$
g_{H g g}=\frac{g_{s}^{2}}{16 \pi^{2}} I_{f}\left(\frac{m_{h}^{2}}{4 m_{t}^{2}}, 0\right),
$$

and the loop functions $I_{f}, I_{w}, I_{w}^{Z}$ were expanded up to $\mathcal{O}\left(m_{t}^{-6} m_{W}^{-6}\right)$, which is formally equivalent to a matching up to $d=11$ in the top-EFT for an on-shell Higgs boson. In version 3.0 this is replaced by a consistent matching up to $\mathcal{O}\left(m_{t}^{-2} m_{W}^{-6}\right)$ that does not rely on the on-shell assumption. The two parameterizations are completely equivalent up to $\mathcal{O}\left(m_{t}^{-4}\right)$ corrections for on-shell Higgs production with no extra jets: the Feynman rule of the $g g h$ interaction is

$$
\begin{aligned}
\vartheta^{\mathcal{Q}_{\mu}\left(p_{1}\right)}= & \frac{4 i}{v}\left[p_{1}^{\nu} p_{2}^{\mu}-\eta^{\mu \nu} p_{1} \cdot p_{2}\right]\left[g_{H g g}^{(1)}-\frac{p_{1} \cdot p_{2}}{m_{t}^{2}} g_{H g g}^{(2)}+\frac{p_{1}^{2}+p_{2}^{2}}{4 m_{t}^{2}} g_{H g g}^{(5)}\right]+ \\
& -\frac{2 i}{v} \frac{p_{1}^{\mu} p_{1}^{\nu} p_{2}^{2}+p_{2}^{\mu} p_{2}^{\nu} p_{1}^{2}-\eta^{\mu \nu} p_{1}^{2} p_{2}^{2}-p_{1}^{\mu} p_{2}^{\nu} p_{1} \cdot p_{2}}{m_{t}^{2}} g_{H g g}^{(4)},
\end{aligned}
$$

with the momenta $p_{1}, p_{2}$ taken to be incoming. In the limit $p_{1,2}^{2}=0, p_{h}^{2}=\left(p_{1}+p_{2}\right)^{2}=m_{h}^{2}$

$$
\underbrace{}_{G_{\nu}\left(p_{2}\right)} \stackrel{\vartheta^{G_{\mu}\left(p_{1}\right)}}{\stackrel{\text { on-shell }}{\longrightarrow}} \frac{4 i}{v}\left[p_{1}^{\nu} p_{2}^{\mu}-\eta^{\mu \nu} \frac{m_{h}^{2}}{2}\right]\left[g_{H g g}^{(1)}-\frac{m_{h}^{2}}{2 m_{t}^{2}} g_{H g g}^{(2)}\right]+\frac{i}{v} \frac{m_{h}^{2}}{m_{t}^{2}} p_{1}^{\mu} p_{2}^{\nu} g_{H g g}^{(4)} \text {. }
$$

The last term vanishes for external gluons and, using eqs. (5.13), (5.14),

$$
g_{H g g}^{(1)}-\frac{m_{h}^{2}}{2 m_{t}^{2}} g_{H g g}^{(2)}=\frac{g_{s}^{2}}{16 \pi^{2}}\left[\frac{1}{3}+\frac{7}{90} \frac{m_{h}^{2}}{4 m_{t}^{2}}\right] .
$$

The terms in brackets reproduce the expansion of the top loop $I_{f}$ (eq. (5.8)) up to $\mathcal{O}\left(m_{t}^{-2}\right)$.

\section{Propagator corrections}

Mass terms and decay widths of the SM particles generally receive corrections from $\mathcal{L}_{6}$ operators. In order to compute amplitudes consistently at $\mathcal{O}\left(\Lambda^{-2}\right)$, these corrections need to be included in the propagators.

In unitary gauge the propagator of a generic unstable vector $V$, scalar $S$ or fermion $\psi$ has the form

$$
\begin{aligned}
P_{V}^{\mu \nu} & =\frac{i}{q^{2}-m_{V}^{2}+i m_{V} \Gamma_{V}}\left(-\eta^{\mu \nu}+\frac{q^{\mu} q^{\nu}}{m_{V}^{2}}\right), \\
P_{S} & =\frac{i}{q^{2}-m_{S}^{2}+i m_{S} \Gamma_{S}}, \\
P_{\psi} & =\frac{i\left(q+m_{\psi}\right)}{q^{2}-m_{\psi}^{2}+i m_{\psi} \Gamma_{\psi}} .
\end{aligned}
$$


In the SMEFT we can write, for each particle,

$$
m=m^{\mathrm{SM}}+\delta m, \quad \Gamma=\Gamma^{\mathrm{SM}}+\delta \Gamma,
$$

where the shifts $\delta m, \delta \Gamma$ collect all the contributions from $d \geq 6$ operators. The corresponding propagator expressions be expanded to linear order in the shifts [98]

$$
P_{V}^{\mu \nu}=P_{V}^{\mu \nu, S M}+\Delta P_{V}^{\mu \nu}, \quad P_{S}=P_{S}^{\mathrm{SM}}+\Delta P_{S}, \quad P_{\psi}=P_{\psi}^{\mathrm{SM}}+\Delta P_{\psi},
$$

where the expressions for $P^{\mathrm{SM}}$ are given by eqs. (6.1)-(6.3) replacing $m \rightarrow m^{\mathrm{SM}}, \Gamma \rightarrow \Gamma^{\mathrm{SM}}$. The corrections read

$$
\begin{aligned}
\Delta P_{V}^{\mu \nu} & =-P_{V}^{\mu \nu, S M} \frac{i m_{V}^{\mathrm{SM}}}{D_{V}\left(q^{2}\right)}\left[\delta \Gamma_{V}+2 i\left(1-\frac{i \Gamma_{V}^{\mathrm{SM}}}{2 m_{V}^{\mathrm{SM}}}\right) \delta m_{V}\right]-2 i \frac{q^{\mu} q^{\nu}}{D_{V}\left(q^{2}\right)} \frac{\delta m_{V}}{\left(m_{V}^{\mathrm{SM}}\right)^{3}}, \\
\Delta P_{S} & =-P_{S}^{\mathrm{SM}} \frac{i m_{S}^{\mathrm{SM}}}{D_{S}\left(q^{2}\right)}\left[\delta \Gamma_{S}+2 i\left(1-\frac{i \Gamma_{S}^{\mathrm{SM}}}{2 m_{S}^{\mathrm{SM}}}\right) \delta m_{S}\right] \\
\Delta P_{\psi} & =-P_{\psi}^{\mathrm{SM}} \frac{i m_{\psi}^{\mathrm{SM}}}{D_{\psi}\left(q^{2}\right)}\left[\delta \Gamma_{\psi}+2 i\left(1-\frac{i \Gamma_{\psi}^{\mathrm{SM}}}{2 m_{\psi}^{\mathrm{SM}}}\right) \delta m_{\psi}\right]+\frac{i \delta m_{\psi}}{D_{\psi}\left(q^{2}\right)},
\end{aligned}
$$

with the shorthand notation

$$
D\left(q^{2}\right)=q^{2}-\left(m^{\mathrm{SM}}\right)^{2}+i m^{\mathrm{SM}} \Gamma^{\mathrm{SM}} .
$$

Note that, since $\Delta P \propto D\left(q^{2}\right)^{-1}$, propagator corrections are expected to be relevant in the on-shell kinematic region and suppressed when the particle is largely off-shell. The dominant contributions are therefore approximated by the on-shell expressions: ${ }^{15}$

$$
\begin{aligned}
\left.\Delta P_{V}^{\mu \nu}\right|_{q^{2}=m^{2}} & =\left.P_{V}^{\mu \nu}\right|_{q^{2}=m^{2}}\left[-\frac{\delta \Gamma_{V}}{\Gamma_{V}^{\mathrm{SM}}}-\left(1+\frac{2 i m_{V}^{\mathrm{SM}}}{\Gamma_{V}^{\mathrm{SM}}}\right) \frac{\delta m_{V}}{m_{V}^{\mathrm{SM}}}\right], \\
\left.\Delta P_{S}\right|_{q^{2}=m^{2}} & =\left.P_{S}\right|_{q^{2}=m^{2}}\left[-\frac{\delta \Gamma_{S}}{\Gamma_{S}^{\mathrm{SM}}}-\left(1+\frac{2 i m_{S}^{\mathrm{SM}}}{\Gamma_{S}^{\mathrm{SM}}}\right) \frac{\delta m_{S}}{m_{S}^{\mathrm{SM}}}\right] \\
\left.\Delta P_{\psi}\right|_{q^{2}=m^{2}} & =\left.P_{\psi}\right|_{q^{2}=m^{2}}\left[-\frac{\delta \Gamma_{\psi}}{\Gamma_{\psi}^{\mathrm{SM}}}-\left(1+\frac{2 i m_{\psi}^{\mathrm{SM}}}{\Gamma_{S}^{\mathrm{SM}}}\right) \frac{\delta m_{\psi}}{m_{\psi}^{\mathrm{SM}}}\right]+\frac{\delta m_{\psi}}{m_{\psi}^{\mathrm{SM}} \Gamma_{\psi}^{\mathrm{SM}}},
\end{aligned}
$$

with

$$
\left.P_{V}^{\mu \nu}\right|_{q^{2}=m^{2}}=\frac{-\eta^{\mu \nu}}{m_{V} \Gamma_{V}},\left.\quad P_{S}\right|_{q^{2}=m^{2}}=\frac{1}{m_{S} \Gamma_{S}},\left.\quad P_{\psi}\right|_{q^{2}=m^{2}}=\frac{q+m_{\psi}}{m_{\psi} \Gamma_{\psi}} .
$$

\subsection{Implementation in SMEFTsim}

SMEFTsim 3.0 implements propagator corrections for the $Z, W, h$ bosons and for the top quark. The user has two alternative options for including them in SMEFT predictions:

(a) using the linearized propagator expressions of eqs. (6.5)-(6.8). In this case the pole of the propagator remains located at $m^{\mathrm{SM}}$, and the dependence on the Wilson coefficients, stemming both from $\delta m$ and $\delta \Gamma$, is linear at the amplitude level. This option is selected fixing linearPropCorrections $=1$ (or any value $\neq 0$ ) in the param_card.

\footnotetext{
${ }^{15}$ Longitudinal contributions for vector bosons were neglected here.
} 
(b) using the propagator expressions in eqs. (6.1)-(6.3), with shifted masses. In this case the pole of the propagator is located at $m=m^{\mathrm{SM}}+\delta m$ while width corrections are entirely dropped. ${ }^{16}$ The dependence on the Wilson coefficients is generally non-linear, as contributions $\propto 1 / C_{\alpha}$ are induced in the amplitude. This is the default option and it's selected with linearPropCorrections $=0$.

While option (a) is recommended for consistency of the EFT expansion, we caution the user that the linearization can be problematic, particularly in the presence of mass corrections. Formally, expanding around the complex pole of the propagator is not a gauge-invariant operation [99-101]. Numerically, significantly large discrepancies between methods (a) and (b) can occur, as illustrated in figure 2 (left) for the case of the $W$ boson. Using linearized propagators leads to sizeable numerical distortions already for $\delta m_{W} / m_{W}^{\mathrm{SM}} \gtrsim$ few $\%$, as the dashed curves show. For comparison, the linear approximation works very well for width corrections up to $\mathcal{O}(10 \%)$, see figure 2 (right). This is partially due to the $W$ boson being narrow in the SM. Adopting a $\left\{m_{W}, m_{Z}, G_{F}\right\}$ input scheme is convenient in this respect, because mass correction effects are entirely avoided. For a more general discussion of the theoretical advantages of this scheme choice, see e.g. refs. [1, 2, 51, 53, 63, 78, 98, 102].

Option (a) is implemented in SMEFTsim following the method outlined in ref. [103], contribution 12: ${ }^{17}$ four dummy fields $Z^{\prime}, W^{\prime}, h^{\prime}, t^{\prime}$ (Z1,W1,H1,t1) are introduced, with propagators $\Delta P_{Z}^{\mu \nu}, \Delta P_{W}^{\mu \nu}, \Delta P_{h}, \Delta P_{t}$. The numerical values assigned to $m^{\mathrm{SM}}, \Gamma^{\mathrm{SM}}$ appearing in these expressions are those of the nominal mass and widths of the dummy fields. The latter, in turn, are internal parameters and defined as equal to the SM masses and widths of the corresponding dynamical particles. The $\delta m, \delta \Gamma$ parameters are also defined as internal parameters, function of the relevant SM couplings and Wilson coefficients.

The dummy states have the same SM interactions as $Z, W, h, t$ and do not enter $d=6$ operators: SMEFTsim contains copies of all SM vertices, with one or more of the standard fields replaced by its dummy counterpart. Vertices with $n$ dummy legs are proportional to $n$ powers of a flag parameter propCorr and have interaction order NPprop $=n$. The numerical value of propCorr is set to 0(1) if linearPropCorrections $=0$ (a non-zero value).

In this way, for instance, linearized $Z$-propagator corrections to $p p \rightarrow \mu^{+} \mu^{-}$can be estimated computing the $p p \rightarrow Z \rightarrow \mu^{+} \mu^{-}$and $p p \rightarrow Z^{\prime} \rightarrow \mu^{+} \mu^{-}$amplitudes, and using the interaction order NPprop to isolate the pure SM/interference/quadratic contributions as detailed in section 8.3.

Note that linearized propagator corrections are available only in the UFO models, as the propagators are modified directly in the propagators.py file and not in FeynRules. ${ }^{18}$

Mass and width corrections implemented. All the mass and width shifts implemented in SMEFTsim are computed to $\mathcal{O}\left(\Lambda^{-2}\right)$, i.e. linearly in the Wilson coefficients. Be-

\footnotetext{
${ }^{16}$ The implementation of EFT-corrected decay widths has been avoided to prevent potential conflicts with the treatment of widths in Monte Carlo generators.

${ }^{17}$ I thank O. Mattelaer for pointing me to this reference.

${ }^{18}$ The expressions in propagators . py differ by an overall $i$ factor from those in (6.6), (6.7), because an $i$ is conventionally added by ALOHA upon parsing the UFO model [104].
} 

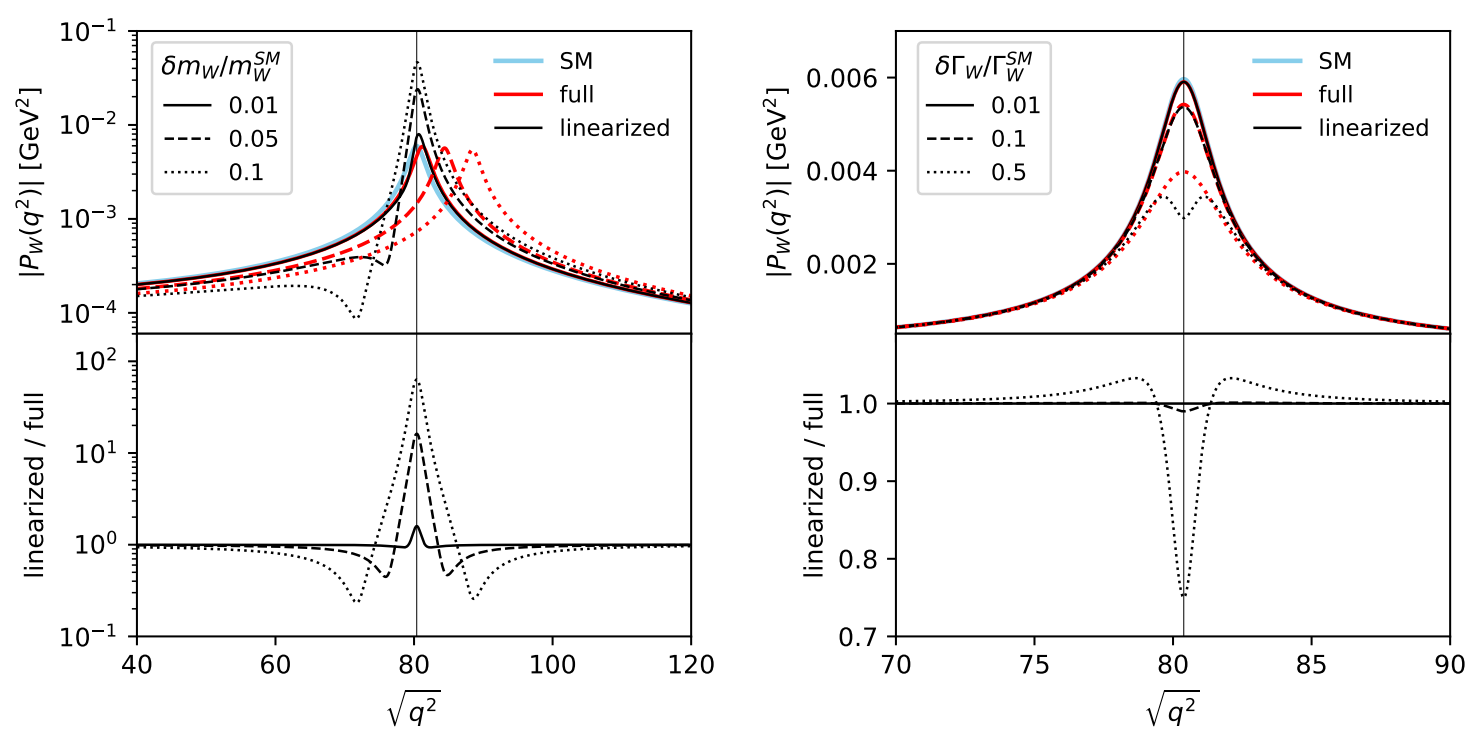

Figure 2. Illustrative comparison of full and linearized $W$ propagators varying $\delta m_{W}$ (left) and $\delta \Gamma_{W}$ (right). The upper panels show the absolute value of the form factor of the transverse propagator $\left|P_{W}\left(q^{2}\right)\right|$ as a function of $\sqrt{q^{2}}$. Red lines are obtained modifying $m_{W}, \Gamma_{W}$ in the full propagator, eq. (6.1), while black lines are obtained linearizing out $\delta m_{W}, \delta \Gamma_{W}$ corrections as in eqs. (6.5), (6.6). The lower panels show the ratio between the two curves. The thin vertical line marks $\sqrt{q^{2}}=$ $m_{W}^{\mathrm{SM}}=80.387 \mathrm{GeV}$. The light blue curve in the upper plots marks for reference the SM behavior $\left(\delta \Gamma_{W}=0=\delta m_{W}\right)$. Solid, dashed and dotted lines correspond to three different sizes of $\delta m_{W}$ and $\delta \Gamma_{W}$, with the same coding in upper and lower panels. In the right panel, the black, red and light blue solid lines are indistinguishable.

cause $m_{Z}, m_{h}$ and $m_{t}$ are taken as input parameters,

$$
\delta m_{Z} \equiv 0, \quad \delta m_{h} \equiv 0, \quad \delta m_{t} \equiv 0 .
$$

The $\delta m_{W}$ correction is non-vanishing only in the $\left\{\alpha_{\mathrm{em}}, m_{Z}, G_{F}\right\}$ scheme, and the expression was given in eq. (4.34). Decay width corrections for the $Z, W$ bosons and for the top quark are defined as

$$
\delta \Gamma_{Z}=\Gamma_{Z}^{S M, \text { best }}\left[\frac{\delta \Gamma_{Z}}{\Gamma_{Z}^{S M}}\right]_{\text {tree }}, \quad \delta \Gamma_{W}=\Gamma_{W}^{S M, \text { best }}\left[\frac{\delta \Gamma_{W}}{\Gamma_{W}^{S M}}\right]_{\text {tree }}, \quad \delta \Gamma_{t}=\Gamma_{t}^{S M, \text { best }}\left[\frac{\delta \Gamma_{t}}{\Gamma_{t}^{S M}}\right]_{\text {tree }},
$$

with

$$
\Gamma_{Z}^{S M, \text { best }}=2.4952 \mathrm{GeV}, \quad \Gamma_{W}^{S M, \text { best }}=2.085 \mathrm{GeV}, \quad \Gamma_{t}^{S M, \text { best }}=1.33 \mathrm{GeV},
$$

the loop-improved SM predictions $[82,105]$. These are free parameters in the models, that can be modified by the user. The quantities $\left[\delta \Gamma / \Gamma^{\mathrm{SM}}\right]_{\text {tree }}$ are calculated at tree level (both numerator and denominator) using the width computation tools in FeynRules [106]. They include all 2-body decays and are extracted in the limit $V_{C K M}=\mathbb{1}$, with all fermion masses set to zero, except those of the $b$ and $t$ quarks. Analytic expressions are given in appendix A. 


\begin{tabular}{|cl|cl|cl|}
\hline$f$ & $\mathrm{Br}_{h \rightarrow f}^{S M, \text { best }}$ & $f$ & $\mathrm{Br}_{h \rightarrow f}^{S M, \text { best }}$ & $f$ & $\mathrm{Br}_{h \rightarrow f}^{S M, \text { best }}$ \\
\hline$\gamma \gamma$ & $2.27 \times 10^{-3}$ & $Z \gamma$ & $1.541 \times 10^{-3}$ & $g g$ & 0.0818 \\
$\bar{b} b$ & 0.5809 & $\bar{c} c$ & 0.02884 & $\tau^{+} \tau^{-}$ & 0.06256 \\
$l l \bar{\nu} \nu$ & 0.0256 & $\bar{u} u \bar{d} d$ & 0.1097 & $l^{-} \bar{\nu} \bar{d} u+$ h.c. & 0.1062 \\
\hline
\end{tabular}

Table 6. Numerical values of the Higgs boson branching ratios employed in the definition of $\delta \Gamma_{h}$, eq. (6.17). The values for 2-body decays are taken from ref. [107], with $m_{h}=125.09 \mathrm{GeV}$. The values in the last line are estimated computing the partial widths with Prophecy4f 2.0 [108] and normalizing their sum to $\mathrm{Br}_{h \rightarrow 4 f}^{S M \text {,best }}=0.24161$ [107]. They include only charged current contributions and are summed over all allowed flavor combinations.

The correction to the total Higgs width is computed using individual $K$-factors for each decay channel, as in ref. [57]: ${ }^{19}$

$$
\delta \Gamma_{h}=\Gamma_{h}^{S M, \text { best }} \sum_{f} \operatorname{Br}_{h \rightarrow f}^{S M, \text { best }}\left[\frac{\delta \Gamma_{h \rightarrow f}}{\Gamma_{h \rightarrow f}^{S M}}\right]_{\text {tree }}, \quad \Gamma_{h}^{S M, \text { best }}=4.07 \mathrm{MeV},
$$

with $f$ running over the set $\left\{\gamma \gamma, Z \gamma, g g, b \bar{b}, c \bar{c}, \tau^{+} \tau^{-}\right\}$plus the allowed 4-fermion channels. In the SMEFTsim implementation, only 4 -fermion decays proceeding via charged currents $\left(h \rightarrow W W^{*} \rightarrow 4 f\right)$ are retained, in order to simplify the analytic expressions. Channels mediated by neutral bosons $\left(h \rightarrow Z Z^{*}, Z \gamma^{*}, \gamma^{*} \gamma^{*}, g^{*} g^{*} \rightarrow 4 f\right)$ give subdominant corrections, that are estimated in a $3-5 \%$ change to the dependence on $\bar{C}_{H W}, \bar{C}_{H B}, \bar{C}_{H D}$ and a change $\lesssim 1 \%$ for the other Wilson coefficients [57].

$\Gamma_{h}^{S M \text {,best }}$ is a free parameter in the models and can be modified by the user. The best-fit branching ratios, instead, are embedded numerically in the $\delta \Gamma_{h}$ expressions and cannot be changed. The values employed are reported in table 6 . The relative deviations $\left[\delta \Gamma_{h \rightarrow f} / \Gamma_{h \rightarrow f}^{\mathrm{SM}}\right]_{\text {tree }}$ for 2-body decays are computed with the FeynRules tools, retaining the full dependence on all the relevant fermion masses and Yukawa couplings. If a given Yukawa coupling $y_{f}$ is set to zero in the param_card, all contributions to $\delta \Gamma_{h}$ originating from the $h \rightarrow f \bar{f}$ decay channel are dropped. For the $h \rightarrow 4 f$ channels we take the analytic results of ref. [57], that neglect all fermion masses and quark mixings. Note that the results in ref. [57] were given for the $\mathrm{U}(3)^{5}$ flavor symmetric case, and they have been generalized to the other flavor assumptions in SMEFTsim. Full analytic results for SMEFT corrections are reported in appendix A.

\section{$7 \quad$ Usage in Mathematica}

The FeynRules files in SMEFTsim can be imported in Mathematica [109] and used to print out analytic expressions for the Feynman rules and Lagrangian terms. A template Mathematica notebook is available at the GitHub repository, with examples of usage of the

\footnotetext{
${ }^{19}$ This normalization choice is due to radiative corrections affecting the various channels in significantly different ways. For comparison, in the case of $Z, W$ decays, using individual $K$-factors leads to variations $\lesssim 2 \%$ in the Wilson coefficient dependence compared to an overall rescaling. The top case is trivial, as there is only one relevant decay channel $t \rightarrow b W^{+}$. In the Higgs case, due to the heterogeneity of the relevant decay processes, the discrepancy between the two normalizations is of order $20-50 \%$.
} 
code in different setups. The functionalities are the standard FeynRules ones. However, since SMEFTsim is an unusually complex model, some recommendations are in order.

Before importing the model, the user must specify a flavor setup and EW input scheme choice. For instance, after loading FeynRules:

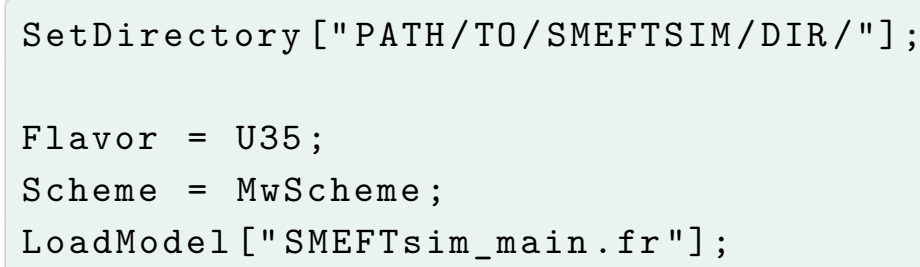

Allowed options for Flavor are general, U35, MFV, top, topU31. Allowed options for Scheme are alphaScheme, MwScheme. The loading time varies between flavor assumptions and can take up to a few seconds. ${ }^{20}$

The FeynRules code is split over different files that contain the required operators, parameters and Lagrangian definitions. The implementation is such that only the objects matching the selected flavor structure and EW input scheme are defined upon loading. In all models, the following Lagrangians are defined (definitions were given in section 1):

- LGauge $=\mathcal{L}_{\text {gauge }}$. Contains the SM terms plus the linearized SMEFT corrections due to field redefinitions and input parameter shifts.

- LGaugeP. Same as LGauge, but with at least one $W$ or $Z$ boson replaced with the corresponding dummy field W1, Z1.

- LHiggs $=\mathcal{L}_{\text {Higgs }}$. Contains the SM terms plus the linearized SMEFT corrections due to field redefinitions and input parameter shifts.

- LHiggsP. Same as LHiggs, but with at least one $W, Z$ or Higgs boson replaced with the corresponding dummy field W1, Z1, H1.

- LFermions $=\mathcal{L}_{\text {fermions }}$. Contains the SM terms plus the linearized SMEFT corrections due to field redefinitions and input parameter shifts.

- LFermionsP. Same as LFermions, but with at least one top quark or $W, Z$ boson replaced with the corresponding dummy field $\mathrm{t} 1$, W1, Z1.

- LYukawa $=\mathcal{L}_{\text {Yukawa }}$. Contains the SM terms plus the linearized SMEFT corrections due to field redefinitions and input parameter shifts.

- LYukawaP. Same as LYukawa, but with at least one top quark or Higgs boson replaced with the corresponding dummy field $t 1, \mathrm{H} 1$.

- $\mathrm{LSM}=\mathcal{L}_{\mathrm{SM}}$. The SM Lagrangian without any SMEFT correction.

\footnotetext{
${ }^{20}$ All the timings indicated in this section refer to a four-core laptop, with FeynRules calculations parallelized.
} 
- LSMlinear. The SM lagrangian plus the linearized SMEFT corrections due to field redefinitions and input parameters shifts.

- LSMIoop $=\mathcal{L}_{\text {SMhloop }}$ as defined in eq. (5.1).

- LSMloopP. Same as LSMloop, but with at least one Higgs or $Z$ boson replaced with the corresponding dummy field $\mathrm{H} 1, \mathrm{Z1}$.

- LSMincl = LSMlinear + LSMloop

- L6cl [n] $=\mathcal{L}_{6}^{(n)}$ with $\mathrm{n}=1 \ldots 8$.

For class 4, the definition is split into L6cl4, L6cl4cpv containing only the CP-even and -odd terms respectively.

For class 8, sub-Lagrangians L6c18a ... L6c18d are defined in addition.

- L6no4f $=\sum_{n=1}^{7} \mathcal{L}_{6}^{(n)}$

- $\mathrm{L} 6=\sum_{n=1}^{8} \mathcal{L}_{6}^{(n)}$

- $\operatorname{LSMEFT}=$ LSMincl + L6

The last 3 Lagrangians contain extremely long expressions. It is strongly recommended to use them with care and avoid calling these variables unless strictly necessary.

The parameters notation in the code is provided in appendix D. In addition, the following parameters lists are defined in all models:

- wC6. The list of all Wilson coefficients.

In the general model the list WC6indices is defined in addition. In this case WC6 contains e.g. cHuIm11, cHuIm33, while WC6indices contains $\mathrm{cHu}\left[\mathrm{ff} 1_{-}, \mathrm{ff} 2_{-}\right]$with blank flavor indices.

- shifts. The list of all shift parameters, such as dGf, dMZ2, dgw, dg1 etc. The complete list is given in table 17 .

- d6pars. List of all SMEFT quantities, including Wilson coefficients with and without free indices, and shifts.

Two handy functions are also defined:

- LinearWC $\left[\mathrm{x}_{-}\right]$. Expands the expression $\mathrm{x}$ to linear order in the SMEFT parameters (Wilson coefficients and shifts).

- SMlimit[x_]. Returns the SM limit of the expression $\mathrm{x}$, setting to zero all the d6pars.

- relativeVariation[x_]. Returns x / SMlimit [x].

- SimplifyWC $\left[\mathrm{x}_{-}\right]$. Returns the expression $\mathrm{x}$ in a form that collects the contributions from each SMEFT parameter (both Wilson coefficients and shifts). 
SetDirectory ["PATH / TO / SMEFTSIM/DIR "];

NotebookEvaluate ["SMEFTsim_SMHIoop_FRs.nb"];

Without producing any output, this will define the objects lhloop5S, 1hloop5PS. The former is a list of all Feynman rules from $\mathcal{L}_{\text {SMhloop }}$, for vertices with up to 5 legs. The latter is the same, but with at least one Higgs boson replaced by the dummy field H1. For instance, the first entry is the $h \gamma \gamma$ vertex:

\section{$\ln [\circ]:=$ Lhloop5S[[1]]}

Out $\left[\circ=\left\{\{\{\mathrm{A}, 1\},\{\mathrm{A}, 2\},\{\mathrm{H}, 3\}\}, \frac{4 i \mathrm{~g}_{\mathrm{HYY}} \mathrm{p}_{1}^{\mu_{2}} \mathrm{p}_{2}^{\mu_{1}}}{\hat{v}}-\frac{4 i g_{H Y Y} \eta_{\mu_{1}, \mu_{2}} \mathrm{p}_{1} \cdot \mathrm{p}_{2}}{\hat{v}}\right\}\right.$

and

$\ln [\circ]:=$ Lhloop5PS[[1]]

Out[o $]=\left\{\{\{\mathrm{A}, 1\},\{\mathrm{A}, 2\},\{\mathrm{H} 1,3\}\}, \frac{4 i \mathrm{~g}_{\mathrm{HYV}} \Delta_{\text {prop }} . \mathrm{p}_{1}^{\mu_{2}} \mathrm{p}_{2}^{\mu_{1}}}{\hat{\mathrm{v}}}-\frac{4 i g_{\mathrm{HYV}} \Delta_{\text {prop. }} \eta_{\mu_{1}, \mu_{2}} \mathrm{p}_{1} \cdot \mathrm{p}_{2}}{\hat{\mathrm{v}}}\right\}$

is explicitly proportional to the propCorr parameter (shown here as $\Delta_{\text {prop. }}$ ), see section $6.1,8.3$.

Finally, it is not recommended to export the UFO models independently, unless only a small subset of operators is included. The UFOs provided in the GitHub repository have been exported in a specific, optimized way and the python files have been manipulated a posteriori in order to introduce the modified form of the propagators and to define the Higgs decay width such that the fermionic decay contributions to $\delta \Gamma_{h}$ is removed whenever the corresponding Yukawa coupling is set to zero. The notebook with the original export procedure is available upon request.

\section{Usage in MadGraph5_aMC@NLO}

This section provides recommendations for the use of SMEFTsim in MadGraph5_aMC@NLO. It is in no way meant as a manual for the functionalities of MadGraph5_aMC@NLO itself, for which we defer the reader to the appropriate references, see e.g. [46, 110, 111].

The SMEFTsim package provides 10 pre-exported UFO models, one for each flavor setup and input parameter scheme. Each of them contains the full $\mathcal{L}_{\text {SMEFT }}$ defined in section 1 and 3 and $\mathcal{L}_{\text {SMhloop }}$ defined in section 5 . The manipulations and redefinitions described in section 2 and 4 have been consistently applied. The vertices contained in the models are derived in unitary gauge and the ghost fields have been removed: SMEFTsim is designed for LO event generation and does not support the NLO syntax. A list of the SMEFT parameters defined in the codes is provided in appendix $\mathrm{D}$, with a mapping to the notation used in this notes. All the UFO models have been validated following the recommendations in ref. [47], as detailed in appendix F. 
Although the selection of an appropriate model is of course up to the taste of the user, each flavor setup is meant to optimize the parameterization of a certain class of effects in the SMEFT. For instance, the top and topU31 models are designed to single out the couplings of the top and bottom quarks [36], and they only differ in that the top case provides more freedom to distinguish the lepton flavors. The U35 models allow one to work with a minimal number of parameters and are recommended for flavor-blind processes or whenever the flavor structure can be assumed to be strictly SM-like. At the other side of the spectrum, the general models provide maximal freedom and can be used to study flavor-violating processes or to realize arbitrary flavor structures beyond those implemented. An operator by operator comparison of the different flavor structures is provided in appendix $\mathrm{C}$.

As discussed in section 6 , the use of the $\left\{m_{W}, m_{Z}, G_{F}\right\}$ input scheme is particularly recommended for processes involving $W$ bosons, as it avoids the problematic introduction of SMEFT corrections to the $W$ pole mass. The $\left\{\alpha_{\mathrm{em}}, m_{Z}, G_{F}\right\}$ and $\left\{m_{W}, m_{Z}, G_{F}\right\}$ input scheme implementations are expected to give results that differ most significantly in the de-

pendence on the Wilson coefficients $C_{H W B}, C_{H D}, C_{H l}^{(3)}, C_{l l}^{\prime}$ (or $\left(C_{H l}^{(3)}\right)_{11},\left(C_{H l}^{(3)}\right)_{22},\left(C_{l l}\right)_{1221}$ in models with explicit flavor indices) and in the presence/absence of corrections to either $m_{W}$ or $\alpha_{\mathrm{em}}$, as described in section 4.2. Further numerical differences affecting both EFT and SM predictions can be present, due to the different definition of the SM parameters in the two cases. These are generally subleading.

\subsection{Parameter cards and restrictions}

The model parameters are grouped in blocks, that are explicitly shown in the parameter cards. Besides the usual ones (SMINPUTS, MASS, DECAY, YUKAWA, CKMBLOCK), the parameter card of each SMEFTsim UFO model contains the blocks:

SMEFTCUTOFF - the parameter $\Lambda$. By default this is $1 \mathrm{TeV}$.

SMEFT - the CP-conserving Wilson coefficients, with default value 0.

SMEFTCPV — the CP-violating Wilson coefficients, with default value 0.

This block is absent in the MFV models.

SMEFTFV - the $\left(\Delta C_{\alpha}\right)$ parameters of the MFV setup, with default value 0.

This block is only present in MFV models.

SWITCHES - the parameter linearPropCorrections, that can be used to switch ON/OFF the linearization of SMEFT corrections in the propagators. The default value is 0 $(\mathrm{OFF})$.

The SMINPUTS block contains $G_{F}, \alpha_{s}$ and either $\alpha_{\mathrm{em}}$ or $m_{W}$ depending on the input scheme. 
The use of restriction cards allows one to reduce the number of diagrams generated for a given process. Two restriction cards are provided by default with each UF0:

- restrict_massless.dat. The masses and Yukawa couplings of all fermions, except the top and bottom quarks, are set to 0. The CKM matrix is set to the identity. The Wilson coefficients are set to arbitrary numerical values.

- restrict_SMlimit_massless.dat. As in restrict_massless.dat, but with all Wilson coefficients set to 0 .

The restrictions should be applied at the stage where the model is imported, e.g.:

import model SMEFTsim_U35_MwScheme_UFO-massless

In this way, all the parameters that are set to either 0 or 1 in the restriction are fixed to their value and cannot be edited further. Sets of parameters that are assigned an identical value in the restriction are fixed to be identical: while their numerical value can still be edited, they cannot be disentangled from one another. Diagrams that are proportional to a vanishing parameter will not be generated.

The use of one of the massless restrictions is recommended for LHC studies, because it simplifies significantly the calculations. There are of course several possible strategies for the use of these restrictions in MadGraph5_aMC@NLO: for instance, one can create a modified version of restrict_SMlimit_massless.dat turning on one Wilson coefficient with some arbitrary value $\neq 0$. Importing the model with this modified restriction allows one to generate events with the chosen coefficient only, while all the other operators are forbidden. Alternatively, if the model is imported with restrict_massless.dat, all the Wilson coefficients are retained: all the allowed SMEFT diagrams will be generated and all the parameters can be freely edited at the event generation stage. Note that, to achieve this, all the Wilson coefficients in restrict_massless.dat are assigned different non-vanishing and non-unitary values, ${ }^{21}$ that will need to be changed prior to the event generation. To simplify this operation, a "restricted" parameter card param_card_massless.dat is provided in the UFO, where all the Wilson coefficients are set to 0 . This card can be directly copied in the PROC/Cards/ directory of the exported process and modified at will.

\subsection{Interaction orders}

A standard feature of UFO models is that every coupling parameter is assigned an interaction order, i.e. a "flag" that provides control on the number of coupling insertions in generated Feynman diagrams. Each parameter carries an arbitrary number of interaction orders.

\footnotetext{
${ }^{21}$ In previous versions of SMEFTsim, the Wilson coefficients in the restriction cards were all set to the special value $9.999999 \mathrm{e}-01$, that in principle allows one to set the parameters to 1 without fixing their value. However, this syntax is not fully supported by MadGraph5_aMC@NLO, and is occasionally source of unexpected numerical behavior in UFO models with a very large number of parameters, such as SMEFTsim general or MFV.
} 


\begin{tabular}{|c|c|}
\hline Order & Parameters assigned \\
\hline & $e, g_{W}, g_{1}, Y_{\psi}$ \\
\hline $\mathrm{QED}=2$ & $G_{F}, \hat{\lambda}, \alpha_{\mathrm{em}}$ \\
\hline $\mathrm{QED}=-1$ & $\hat{v}, \Lambda$ \\
\hline $\mathrm{QCD}=1$ & $g_{s}$ \\
\hline $\mathrm{QCD}=2$ & $\alpha_{s}$ \\
\hline SMHLOOP $=1$ & $g_{H \gamma \gamma}, g_{H Z \gamma}, g_{H g g}^{(1)} \ldots g_{H g g}^{(5)}$ \\
\hline$N P=1$ & all Wilson coefficients and shifts, except propCorr \\
\hline $\mathrm{NPcpv}=1 \quad(\star)$ & all CP-violating Wilson coefficients \\
\hline $\operatorname{NPfv}=1 \quad(\star)$ & all $(\Delta C)$ parameters in MFV models \\
\hline $\mathrm{NPc}[\mathrm{a}]=1 \quad(\star)$ & all the $\mathrm{CP}$ components and flavor indices of the Wilson coefficient $C_{\alpha}$ \\
\hline NPprop $=1 \quad(\star)$ & propCorr \\
\hline
\end{tabular}

Table 7. Interaction orders defined in the SMEFTsim UFO models. Those marked with a $(\star)$ are new in version 3.0. The string [a] stands for a generic root name of a Wilson coefficient, as listed in appendix D.

\subsubsection{Definitions}

In the SMEFTsim UFO models the interaction orders are assigned as reported in table 7.

The orders QED and QCD are assigned as customary in the standard SM UFO implementations, with the exceptions of the SMEFT cutoff $\Lambda$, that has been assigned QED $=-1$ such that the combination $(\hat{v} / \Lambda)$ is order-less, and of the Wilson coefficient $C_{H}$, that has been assigned $\mathrm{QED}=1$. This prevents the $C_{H}$ correction to the $h^{3}$ interaction, that is proportional to $\hat{v}^{3} C_{H} / \Lambda^{2}$ (see eq. (4.44)), from having overall order QED $=-1$.

The interaction order SMHLOOP labels the SM loop-generated Higgs interactions introduced in section 5. Since by definition they are proportional to the SM gauge couplings, the $g_{H g g}^{(k)}$ parameters additionally carry QCD=2 and the $g_{H \gamma \gamma}, g_{H Z \gamma}$ parameters carry QED=2.

The interaction order NP (New Physics) is assigned to all the Wilson coefficients and shifts indistinctly. In addition, starting from version 3.0, individual interaction orders have been introduced for each effective operators. The same order NPc [a] is assigned to all the associated $\mathrm{CP}$-conserving and violating parameters, irrespective of the flavor indices carried. For instance, in the top models, the parameters $\operatorname{Re}\left(C_{e H}\right)_{p p}, \operatorname{Im}\left(C_{e H}\right)_{p p}$ for $p=\{1,2,3\}$ all have order $\mathrm{NPceH}=1$. In the U35, MFV, top and topU31 models, distinct interaction orders are assigned to independent flavor contractions. For instance $C_{l l}$ and $C_{l l}^{\prime}$ have orders NPcll and NPcll1 respectively. In the top models, the parameters $\left(C_{l l}\right)_{p p r r}$ have order NPcll, while the $\left(C_{l l}\right)_{\text {prrp }}$ contractions have order NPcll1, etc. In most cases the label [a] coincides with the name root of the associated Wilson coefficient, that can be read off from the tables in appendix D. If in doubt, the user can resort to the .fr source files or check explicitly the couplings.py file to identify the exact orders assigned to a given parameter or coupling. 
All the CP-violating parameters, that belong to the SMEFTCPV block, have an order NPcpv=1. Analogously, all the $\left(\Delta C_{\alpha}\right)$ quantities in the MFV models, that belong to the SMEFTFV block, have an order NPf $\mathrm{v}=1$.

Finally, the order NPprop labels the interactions of the dummy fields $W^{\prime}, Z^{\prime}, h^{\prime}, t^{\prime}$ carrying linearized propagator corrections, see section 6.1. It is carried by a dummy internal parameter propCorr that only takes values $0 / 1$, when the linearPropCorrections switch is set to 0/a nonzero value. Its application is discussed in the next subsection. By default, the interaction order NPprop is "switched off", as it is assigned an upper limit of 0 interactions, that can be lifted as shown below. No upper limit is set for the other orders.

The interaction orders SMHLOOP, NP, NPprop, NPcpv, NPfv have been assigned hierarchy 99. MadGraph5_aMC@NLO will therefore generally avoid insertions of the associated vertices, unless these orders are specified.

\subsubsection{Recommended use}

Interaction orders are specified at the stage of process generation in MadGraph5_aMC@NLO, e.g.:

generate $p p>m u+m u-S M H L O O P=0$ NPprop=0 NP=1 NP^2==1

where $=$ is equivalent to $<=$, while $==$ selects uniquely the order specified. The syntax $\mathrm{XX}=\mathrm{n}$ acts at the amplitude level, i.e. it specifies the total number of couplings with order $\mathrm{XX}$ to be inserted in each Feynman diagram. The syntax $\mathrm{XX}^{\wedge} 2$ acts instead at the squared amplitude level. This functionality works very nicely for EFT studies, as it allows one to disentangle contributions at different orders in the expansion.

Although a priori SMEFTsim can be used for computations to any allowed order in $\Lambda$, it implements the SMEFT Lagrangian consistently expanded only up to $\mathcal{O}\left(\Lambda^{-2}\right)$. This means that any SMEFTsim prediction beyond this order is necessarily incomplete in the Effective Theory. It is worth noting that this statement does not concern only higher dimensional operators in $\mathcal{L}_{\mathrm{SMEFT}}$, but also affects the dependence on some of the Wilson coefficients in $\mathcal{L}_{6}$. For instance, it was stressed at multiple stages in sections 2 and 4 that terms of order $\Lambda^{-4}$ or higher were neglected in the field and parameter redefinitions performed, as well as in the treatment of input parameters. The impact of these $\mathcal{L}_{6}$ contributions has been discussed in ref. [112] for the case of $\mathcal{O}\left(\Lambda^{-4}\right)$ corrections to $1 \rightarrow 2$ decays, using the geoSMEFT formalism [62].

Complete results truncated at $\mathcal{O}\left(\Lambda^{-2}\right)$ can be obtained with the syntax $\mathrm{NP}<=1 \mathrm{NP}^{\wedge} 2<=1$, that retains only SM plus SM- $\mathcal{L}_{6}$ interference contributions. Contributions of order $\Lambda^{-4}$ stemming from the square of an $\mathcal{O}\left(\Lambda^{-2}\right)$ amplitude, although incomplete, are also commonly included in the SMEFT calculations: they are selected with $\mathrm{NP}=1 \mathrm{NP} \mathrm{N}^{\wedge} 2==2$ or just $\mathrm{NP}==1$. For the reasons above, it is generally recommended to use the specification $\mathrm{NP}<=1$ (or NP=1) for any process, to limit the number of EFT insertions to one per Feynman diagram. A generic observable computed in this way will have the form

$$
\sigma=\sigma_{\mathrm{SM}}+\sum_{\alpha} \sigma_{\alpha} \bar{C}_{a}+\sum_{\alpha, \beta} \sigma_{\alpha \beta} \bar{C}_{\alpha} \bar{C}_{\beta},
$$




\begin{tabular}{|c|c|c|c|c|c|c|}
\hline & $\sigma_{\mathrm{SM}}$ & $\sigma_{\alpha}$ & $\sigma_{\beta}$ & $\sigma_{\alpha \alpha}$ & $\sigma_{\beta \beta}$ & $\sigma_{\alpha \beta}$ \\
\hline $\mathrm{NP}=0$ & $\checkmark$ & & & & & \\
\hline$N P<=1$ & $\checkmark$ & $\checkmark$ & $\checkmark$ & $\checkmark$ & $\checkmark$ & $\checkmark$ \\
\hline$N P==1$ & & & & $\checkmark$ & $\checkmark$ & $\checkmark$ \\
\hline $\mathrm{NP}<=1 \quad \mathrm{NP}^{\wedge} 2<=1$ & $\checkmark$ & $\checkmark$ & $\checkmark$ & & & \\
\hline $\mathrm{NP}<=1 \quad \mathrm{NP}^{\wedge} 2==1$ & & $\checkmark$ & $\checkmark$ & & & \\
\hline $\mathrm{NP}<=1 \mathrm{NPc}[a]^{\wedge} 2<=1$ & $\checkmark$ & $\checkmark$ & & & & $\checkmark$ \\
\hline $\mathrm{NP}<=1 \mathrm{NPc}[\mathrm{a}]^{\wedge} 2<=1 \mathrm{NPc}[\mathrm{b}]^{\wedge} 2<=1$ & $\checkmark$ & $\checkmark$ & $\checkmark$ & & & $\checkmark$ \\
\hline$N P<=1 \quad N P c[a]==1$ & & $\checkmark$ & & $\checkmark$ & & \\
\hline $\mathrm{NP}<=1 \mathrm{NPc}[a]^{\wedge} 2==1$ & & $\checkmark$ & & & & $\checkmark$ \\
\hline $\mathrm{NP}<=1 \mathrm{NPc}[\mathrm{a}]^{\wedge} 2==2$ & & & & $\checkmark$ & & \\
\hline $\mathrm{NP}<=1 \quad \mathrm{NP}^{\wedge} 2==1 \quad \mathrm{NPc}[\mathrm{a}]^{\wedge} 2==1$ & & $\checkmark$ & & & & \\
\hline$N P<=1 \quad N N^{\wedge} 2==2 N P c[a]^{\wedge} 2==1$ & & & & & & $\checkmark$ \\
\hline
\end{tabular}

Table 8. Examples of interaction-order syntax that select different EFT contributions to a generic observable with the dependence given in eq. (8.1), for the case of 2 Wilson coefficients $C_{\alpha}, C_{\beta}$.

where $\sigma_{\mathrm{SM}}, \sigma_{\alpha}, \sigma_{\alpha \beta}$ intuitively denote the $\mathrm{SM}$, interference and quadratic contributions respectively. $\sigma_{\mathrm{SM}}$ and $\sigma_{\alpha \alpha}$ are always positive quantities, while $\sigma_{\alpha}, \sigma_{\alpha \beta}$ with $\alpha \neq \beta$ can take negative values. Table 8 shows examples of how the interaction order syntax can be used to disentangle these contributions, for a simple case with two Wilson coefficients. The expressions directly generalize if three or more parameters are present.

Finally, as discussed in section 5, the loop-generated SM Higgs couplings implemented in SMEFTsim are defined in the $m_{t} \rightarrow \infty$ limit, and their use should be limited to (on-shell) Higgs production and decay processes. Outside of this regime, it is strongly recommended to use SMHLOOP=0.

\subsection{Propagator corrections and decay widths}

As discussed in section 6, SMEFT corrections are generally present in the propagators of unstable particles, due to $d=6$ operators modifying their masses and/or decay widths. Section 6.1 outlined two alternative methods for estimating these contributions in a given process. In the following we illustrate how they can be implemented in MadGraph5_aMC@NLO.

\subsubsection{Method (a): linearized corrections}

SMEFTsim 3.0 offers the possibility to linearize propagator corrections as in section 6 for the $Z, W, h$ bosons and for the top quark. The implementation relies on the introduction of dummy fields $Z^{\prime}, W^{\prime}, h^{\prime}, t^{\prime}$ whose couplings carry interaction order NPprop, which allows one to single them out. For instance, the syntax

generate $p$ p $>e+e-S M H L O O P=0 \quad N P==1$ NPprop $=0$ 
selects all diagrams with one effective operator in a vertex (including 4-point $\bar{q} q e^{+} e^{-}$interactions) but none in propagators, while

generate $\mathrm{p} \mathrm{p}>\mathrm{e}+\mathrm{e}-\mathrm{SMHLOOP}=0$ NP=0 NPprop<=2 NPprop`2==2

generate $\mathrm{p} p>e+e-\operatorname{SMHLOOP}=0$ NP=0 NPprop $==2$

extract the pure SM- $\mathcal{L}_{6}$ interference $\left(\propto \delta \Gamma_{Z}\right)$ and the quadratic $\left(\propto\left(\delta \Gamma_{Z}\right)^{2}\right)$ contributions respectively from corrections to the $Z$ propagator, ${ }^{22}$ and exclude EFT insertions in vertices. Due to the absence of additional interaction orders, there is unfortunately no equivalent to table 8 in this case: propagator corrections from different operators and EFT orders cannot be disentangled at this level.

Important: in order to avoid unwanted insertions of the dummy fields in standard process generations, the functionality described here has to be activated in 2 steps: (i) in the file coupling_order.py, the expansion_order option for the order NPprop has to be set to a number $\geq 2$ (recommended: 99). The default is 0 , which forbids dummy interactions completely. (ii) The parameter linearPropCorrections in the param_card has to be set to a non-zero value. If this is not the case, dummy vertices will be included in the diagrams, but they will be idle, as they are proportional to propCorr $=0 / 1$ for linearPropCorrections $=0 /$ non-zero.

\subsubsection{Method (b): full corrections}

As an alternative to linearization, propagator corrections can be estimated following more canonical procedures. This generally means computing processes with the propagator forms in eqs. (6.1)-(6.3), with mass and decay parameters that depend explicitly on the Wilson coefficients, to either linear or quadratic order. The resulting process will thus exhibit a non-polynomial dependence on the SMEFT parameters.

The most relevant caveat here is that the implementation of the Wilson coefficient dependence is necessarily different for masses and widths. In the former case, it is possible to define mass parameters as internal and assign them an analytic expression, e.g. MW $=$ MWsm + dMW, with dMW defined as in (4.33). In SMEFTsim the dMW term is only included when linearPropCorrections $=0$, and switched off otherwise. Note also that the expression of a generic $\delta m$ is extracted at the Lagrangian level and is purely of $\mathcal{O}\left(\Lambda^{-2}\right)$.

On the other hand, due to how Monte Carlo generators and their interface to parton shower or decay modules are structured, decay widths cannot be defined as internal parameters in UFO models. Therefore the only way their SMEFT expressions can be inserted in the calculation is by letting MadGraph5_aMC@NLO compute them, by setting the relevant widths to Auto in the param_card [106]. The on-the-fly calculation will include all allowed 2-body decays as well as higher multiplicity decays estimated to be numerically relevant, and it will rely on the pre-computed decay results collected in the file decays.py, which

\footnotetext{
${ }^{22}$ Remember that NPprop counts the number of dummy vertices, so, in this case, the order specified is twice the number of dummy propagators.
} 
include $\mathcal{O}\left(\Lambda^{-4}\right)$ terms. $^{23}$ Note that, with this procedure, the decay widths will need to be re-evaluated every time the value of a relevant Wilson coefficient is modified.

With both mass and width corrections evaluated as above, the denominator of a generic propagator has the form

$$
q^{2}-\left(m^{\mathrm{SM}}+\delta m\right)^{2}+i\left(m^{\mathrm{SM}}+\delta m\right)\left(\Gamma^{\mathrm{SM}}+\delta^{(1)} \Gamma+\delta^{(2)} \Gamma\right),
$$

where $\delta m$ and $\delta^{(1)} \Gamma$ are of $\mathcal{O}\left(\Lambda^{-2}\right)$ and $\delta^{(2)} \Gamma$ is of $\mathcal{O}\left(\Lambda^{-4}\right)$. Eq. (8.2) contains therefore terms up to $\mathcal{O}\left(\Lambda^{-6}\right)$. An observable computed for a process with $k$ internal lines corrected in this way, will contain terms up to $\mathcal{O}\left(\Lambda^{-12 k}\right)$ at the denominator.

In principle this functional dependence can be reconstructed fitting the appropriate rational function to a sufficient number of benchmark points. However, it is recommended to reduce the proliferation of higher-order terms in the propagators, by evaluating mass and width corrections separately and by treating propagator corrections to different internal states individually, whenever possible. This is achieved avoiding to switch on at the same time the linearPropCorrections flag (that turns on $\delta m_{W}$ ) and the Auto computation of a decay width, or of two decay widths simultaneously.

\subsection{Example: Higgs production and decay including $W, Z$ propagator corrections}

As a practical example for the use of SMEFTsim in MadGraph5_aMC@NLO, we compute SMEFT corrections to Higgs production and decay processes that are mediated by $W, Z$ exchange, as an illustration of the propagator corrections feature.

\subsubsection{STXS for $\bar{q} q \rightarrow h \bar{q} q$}

We consider two bins of the stage 1.1 Simplified Template Cross Section (STXS) parameterization [113-116], for the EW $\bar{q} q \rightarrow h \bar{q} q$ production channel at low Higgs $p_{T}$.

They are defined by the cuts [115]:

$$
\begin{array}{l|ll}
\text { VBF-like } & 350 \mathrm{GeV}<m_{j j} & p_{T}(h)<200 \mathrm{GeV},\left|y_{h}\right|<2.5 \\
\text { VH-like } & 60 \mathrm{GeV}<m_{j j}<120 \mathrm{GeV} & p_{T}(h)<200 \mathrm{GeV},\left|y_{h}\right|<2.5 .
\end{array}
$$

with $y_{h}$ the rapidity of the Higgs boson. In each bin, the Higgs production cross section in the SMEFT can be parameterized as:

$$
\sigma_{S M E F T}=\sigma_{\mathrm{SM}}+\sum_{\alpha} \sigma_{\alpha} \bar{C}_{\alpha}+\mathcal{O}\left(\Lambda^{-4}\right)
$$

Table 9 reports the values of $\sigma_{\alpha} / \sigma_{\mathrm{SM}}$ for the relevant fermionic operators, computed at parton level using SMEFTsim in the U35 flavor-symmetric, $\left\{m_{W}, m_{Z}, G_{F}\right\}$ input scheme version. The following procedure was followed:

1. 50000 events are generated for each bin in MadGraph5_aMC@NLO, for $\sqrt{s}=13 \mathrm{TeV}$. The syntax used is

\footnotetext{
${ }^{23}$ Since only $1 \rightarrow 2$ decays are included, these results consistently stem from the square of $\mathcal{O}\left(\Lambda^{-2}\right)$ amplitudes. Dummy fields are not included in the pre-computed decay widths.
} 
import model SMEFTsim_U35_MwScheme_UFO-vbf

generate $q \mathrm{q}>\mathrm{h} q \mathrm{q} Q \mathrm{QCD}=0 \quad \mathrm{NP}=0 \quad$ SMHLOOP=0 NPprop=0

where the -vbf flag indicates that the model is imported with a custom restriction card restrict_vbf.dat, that in this case sets to zero the masses and Yukawa couplings of all fermions except the bottom and top quarks, as well as all the Wilson coefficients that are known not to contribute to the process. The remaining ones are set to a random non-zero value in this card. The STXS defining cuts in $p_{T}(h)$ and $y_{h}$ cuts are implemented at the level of the run_card.dat in MadGraph5_aMC@NLO, while the invariant mass cuts are applied when analyzing the events a posteriori. This gives the tree-level SM cross sections

$$
\sigma_{\mathrm{SM}}^{V B F}=1.56 \mathrm{pb}, \quad \sigma_{\mathrm{SM}}^{V H}=0.67 \mathrm{pb} .
$$

2. The events are reweighted using the reweight module in MadGraph5_aMC@NLO [117]. Individual weights are computed for each Wilson coefficient, splitting contributions from operator insertions in the vertices (labeled as "direct") and from insertions in the $W, Z$ propagators (labeled as "propagator"). This is done setting each coefficient to 1 and the SMEFT cutoff scale LambdaSMEFT to $1 \mathrm{TeV}$. For instance, for the $C_{l l}^{\prime}$ parameter, the reweight_card.dat for the direct contributions is

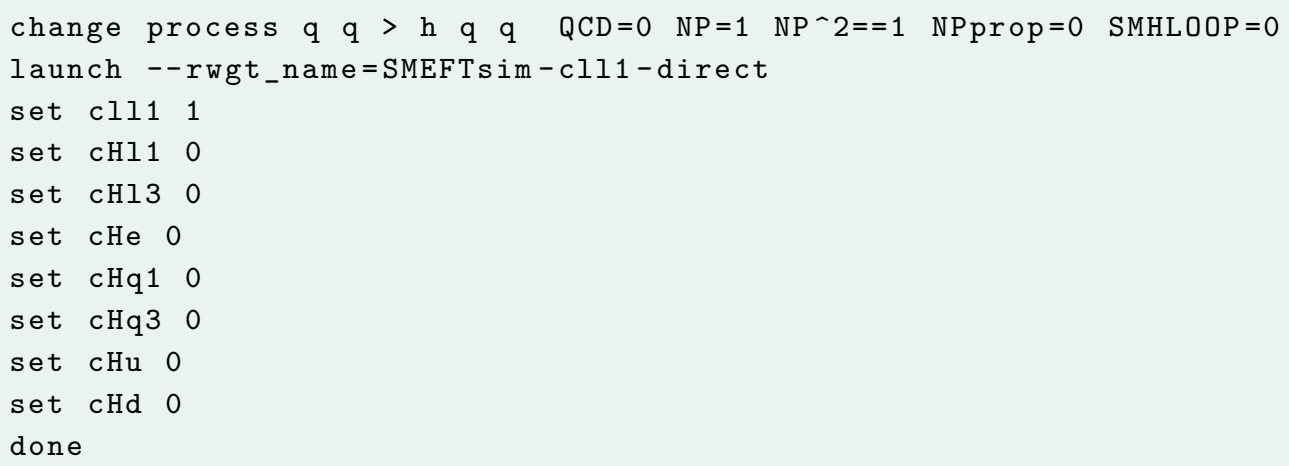

For estimating the pure propagator contributions the first two lines are replaced with

change process $q \mathrm{q}>\mathrm{h} q \mathrm{q}$ QCD=0 $\mathrm{NP}=0$ NPprop=2 NPprop ${ }^{-2}==2$ SMHLOOP=0

launch - -rwgt_name=SMEFTsim-cll1-propagator

Analyzing the reweighted events gives $\left(\sigma_{\alpha} \frac{\hat{v}^{2}}{\Lambda^{2}}\right)$ for each $C_{\alpha}$. The numbers in table 9 are finally obtained dividing by $\sigma_{\mathrm{SM}}$ and normalizing to $\bar{C}_{\alpha}$.

The results show that propagator corrections are negligible in the VBF regime, where the relative SMEFT corrections to the cross section is

$$
\frac{\sigma_{S M E F T}}{\sigma_{\mathrm{SM}}}=1-3 \cdot 10^{-4} \frac{\delta \Gamma_{Z}}{\Gamma_{Z}^{\mathrm{SM}}}-7 \cdot 10^{-4} \frac{\delta \Gamma_{W}}{\Gamma_{W}^{\mathrm{SM}}}+\text { direct. }
$$




\begin{tabular}{|c|rr|rr|rr|}
\hline & \multicolumn{2}{|c|}{$\bar{q} q \rightarrow h \bar{q} q$ VBF-like } & \multicolumn{2}{c|}{$\bar{q} q \rightarrow h \bar{q} q$ VH-like } & \multicolumn{2}{c|}{$h \rightarrow e^{+} e^{-} \mu^{+} \mu^{-}$} \\
\hline & direct & propagators & direct & propagators & direct & propagators \\
\hline $\bar{C}_{H e}$ & & $5.32 \cdot 10^{-5}$ & & 0.0526 & -1.724 & 0.153 \\
$\bar{C}_{H l}^{(1)}$ & & $5.32 \cdot 10^{-5}$ & & 0.0526 & 2.144 & 0.153 \\
$\bar{C}_{H l}^{(3)}$ & -6 & $1.351 \cdot 10^{-3}$ & -6 & 1.258 & -3.856 & 1.147 \\
$\bar{C}_{H q}^{(1)}$ & 0.109 & $-1.363 \cdot 10^{-4}$ & -0.197 & -0.135 & & -0.39 \\
$\bar{C}_{H q}^{(3)}$ & -5.345 & $-1.423 \cdot 10^{-3}$ & 25.66 & -1.329 & & -1.353 \\
$\bar{C}_{H u}$ & -0.323 & $-7.092 \cdot 10^{-5}$ & 1.926 & -0.070 & & -0.203 \\
$\bar{C}_{H d}$ & 0.103 & $5.24 \cdot 10^{-5}$ & -0.608 & 0.0518 & & 0.150 \\
$\bar{C}_{l l}^{\prime}$ & 3 & $-1 . \cdot 10^{-3}$ & 3 & -0.936 & 3 & -0.839 \\
\hline
\end{tabular}

Table 9. Values of $\sigma_{a} / \sigma_{\mathrm{SM}}$ for the relevant fermionic Wilson coefficients $\bar{C}_{\alpha}$ contributing to $\bar{q} q \rightarrow$ $h \bar{q} q$ and $h \rightarrow e^{+} e^{-} \mu^{+} \mu^{-}$. For the first two columns $\sigma$ is the cross section in the VBF-like and VHlike STXS bins defined in the text, while in the third the numbers refer to the partial decay width. The results are given for $\left\{m_{W}, m_{Z}, G_{F}\right\}$ inputs with a $\mathrm{U}(3)^{5}$ flavor symmetry, and neglecting all fermion masses. The "direct" contributions stem from operator insertions in vertices, including parameter shifts, while the "propagator" ones stem from the corrections to the $W, Z$ decay width in internal propagators. The lines highlighted in color are those for which the latter are most relevant.

This is expected, as the intermediate bosons in $t$-channel are mostly off-shell in this process. In the VH bin, on the other hand, one vector boson can be on-shell, which enhances the propagator effects. In this case, the relative SMEFT correction is

$$
\frac{\sigma_{S M E F T}}{\sigma_{\mathrm{SM}}}=1-0.29 \frac{\delta \Gamma_{Z}}{\Gamma_{Z}^{\mathrm{SM}}}-0.65 \frac{\delta \Gamma_{W}}{\Gamma_{W}^{\mathrm{SM}}}+\text { direct }
$$

where the numerical prefactors reflect the proportions of $W$ and $Z$ bosons produced. In fact, the largest numerical effects in table 9 are observed in the operators entering $\delta \Gamma_{W}$.

\subsection{2 $h \rightarrow e^{+} e^{-} \mu^{+} \mu^{-}$}

An analysis of the $Z$-mediated Higgs decay $h \rightarrow e^{+} e^{-} \mu^{+} \mu^{-}$was performed following a procedure analogous to the one described for $\bar{q} q \rightarrow h \bar{q} q$. In the decay case, one $Z$ boson is always on-shell, leading to significant contributions from the intermediate $Z$ propagator. The relative SMEFT correction to the decay width is found to be

$$
\frac{\Gamma_{S M E F T}}{\Gamma_{\mathrm{SM}}}=1-0.84 \frac{\delta \Gamma_{Z}}{\Gamma_{Z}^{\mathrm{SM}}}+\text { direct }
$$

The breakdown into fermionic Wilson coefficients is given in table 9 and it agrees with the analytic results of ref. [57]. 


\section{Summary}

The SMEFTsim package contains models in FeynRules and in the UFO format, that implement the complete Warsaw basis of dimension six operators, under different flavor assumptions and with different choices of the input quantities for the EW sector. Its main scope is the Monte Carlo simulation of LHC processes in the SMEFT, but it can also be employed for simple analytic calculations, exploiting the FeynRules interface in Mathematica.

This work reviewed the theoretical elements that are implemented in SMEFTsim and presented the improvements in version 3.0. The most significant changes compared to previous releases are the addition of two new flavor structures for top quark physics, the implementation of a brand new tool for the inclusion of SMEFT corrections in the propagator of unstable particles and the general improvement of the code, particularly of the parameterization of Higgs-gluon interactions in the SM.

As in previous versions, SMEFTsim 3.0 supports the WCxf exchange format [22]. The corresponding interface will be updated shortly after the code release. Finally, support for the translation of the UFO models to python3 will be provided in the near future.

\section{Acknowledgments}

I would like to thank Sebastian Bruggisser, Ana Cueto, Sally Dawson, Gauthier Durieux, Saskia Falke, Gino Isidori, Olivier Mattelaer, Luca Merlo, Ken Mimasu and Mike Trott for several conversations that inspired and improved this work, Tyler Corbett and Pietro Govoni for their valuable comments on the manuscript. The author acknowledges support by the state of Baden-Württemberg through bwHPC and the German Research Foundation (DFG) through grant no INST39/963-1 FUGG (bwForCluster NEMO).

\section{A Analytic expressions of decay width corrections}

This appendix reports analytic expressions for the decay widths of the $W, Z, h, t$ particles in the SMEFT, that are implemented in the tool documented in section 6.1.

Only linear terms in the Wilson coefficients are retained. CKM mixing and all fermion masses except $m_{b}$ and $m_{t}$ are neglected, unless otherwise specified. Results are reported in the flavor-general setup, and they can be mapped to the symmetric scenarios using the tables in appendix C. We use an input-scheme independent notation. Scheme-specific results can be obtained replacing the generic shifts $\delta g_{W}, \delta g_{1}, \delta m_{W}^{2}$ with the expressions reported in section 4.2.1 or 4.2.2. The quantities $\Delta G_{F}, \Delta m_{Z}^{2}, \Delta \kappa_{H}$ are defined in table 4 . Finally, we use the $\bar{C}$ notation defined in eq. (2.4) and the hat notation defined in section 4 .

\section{A.1 $Z$ boson}

The $Z$ boson couplings to a fermion pair $\psi \bar{\psi}$ are

$$
g_{\psi L}=T_{3}^{\psi}-Q_{\psi} s_{\hat{\theta}}^{2}, \quad g_{\psi R}=-Q_{\psi} s_{\hat{\theta}}^{2},
$$


where $T_{3}^{\psi}= \pm 1 / 2$ is the isospin eigenvalue and $Q_{\psi}$ is the electric charge of the fermion $\psi$. We also define:

$$
\begin{array}{rlrl}
\Delta_{\nu, p p}^{L} & =\left(\bar{C}_{H l}^{(1)}\right)_{p p}-\left(\bar{C}_{H l}^{(3)}\right)_{p p}, & & \\
\Delta_{e, p p}^{L} & =\left(\bar{C}_{H l}^{(1)}\right)_{p p}+\left(\bar{C}_{H l}^{(3)}\right)_{p p}, & & \Delta_{e, p p}^{R}=\left(\bar{C}_{H e}\right)_{p p}, \\
\Delta_{u, p p}^{L} & =\left(\bar{C}_{H q}^{(1)}\right)_{p p}-\left(\bar{C}_{H q}^{(3)}\right)_{p p}, & \Delta_{u, p p}^{R}=\left(\bar{C}_{H u}\right)_{p p}, \\
\Delta_{d, p p}^{L} & =\left(\bar{C}_{H q}^{(1)}\right)_{p p}+\left(\bar{C}_{H q}^{(3)}\right)_{p p}, & \Delta_{d, p p}^{R}=\left(\bar{C}_{H d}\right)_{p p} .
\end{array}
$$

At tree level in the SM, the partial decay width of the $Z$ boson into a $\bar{\psi}_{p} \psi_{p}$ pair, with $\psi=\left\{\nu, l^{-}, u, d\right\}$ and flavor $p$, is

$$
\Gamma_{Z \rightarrow \bar{\psi}_{p} \psi_{p}}^{\mathrm{SM}}=\frac{G_{F} m_{Z}^{3}}{3 \sqrt{2} \pi} N_{C}^{\psi}\left[g_{\psi L}^{2}+g_{\psi R}^{2}\right]
$$

where $N_{c}^{\psi}$ is the number of colors of the fermion species $\psi$.

The relative SMEFT correction to a partial width can be inferred differentiating in the $g_{\psi L}, g_{\psi R}$ couplings and inserting the expressions of their SMEFT shifts:

$$
\begin{gathered}
\delta g_{\psi L}=g_{\psi L} \delta g_{Z}-Q_{\psi} \delta s_{\theta}^{2}-\frac{\Delta_{\psi, p p}^{L}}{2} \\
\delta g_{\psi R}=g_{\psi R} \delta g_{Z}-Q_{\psi} \delta s_{\theta}^{2}-\frac{\Delta_{\psi, p p}^{R}}{2}
\end{gathered}
$$

with

$$
\delta g_{Z}=\frac{\delta g_{W}}{\hat{g}_{W}}+\frac{\delta s_{\theta}^{2}}{2 c_{\hat{\theta}}^{2}}+\frac{s_{\hat{\theta}}}{2 c_{\hat{\theta}}} \bar{C}_{H W B}
$$

Using the expression of $\delta s_{\theta}^{2}$ provided in eq. (4.32), one obtains

$$
\begin{aligned}
\frac{\delta \Gamma_{Z \rightarrow \bar{\psi}_{p} \psi_{p}}}{\Gamma_{Z \rightarrow \bar{\psi}_{p} \psi_{p}}^{\mathrm{SM}}}= & 2 c_{\hat{\theta}}^{2}\left[1+2 s_{\hat{\theta}}^{2} Q_{\psi} \frac{g_{\psi L}+g_{\psi R}}{g_{\psi L}^{2}+g_{\psi R}^{2}}\right] \frac{\delta g_{W}}{\hat{g}_{W}}+2 s_{\hat{\theta}}^{2}\left[1-2 c_{\hat{\theta}}^{2} Q_{\psi} \frac{g_{\psi L}+g_{\psi R}}{g_{\psi L}^{2}+g_{\psi R}^{2}}\right] \frac{\delta g_{1}}{\hat{g}_{1}} \\
& +s_{2 \hat{\theta}}\left[1-c_{2 \hat{\theta}} Q_{\psi} \frac{g_{\psi L}+g_{\psi R}}{g_{\psi L}^{2}+g_{\psi R}^{2}}\right] \bar{C}_{H W B}-\frac{g_{\psi L} \Delta_{\psi, p p}^{L}+g_{\psi R} \Delta_{\psi, p p}^{R}}{g_{\psi L}^{2}+g_{\psi R}^{2}}
\end{aligned}
$$

Flavor violating decays are absent at $\mathcal{O}\left(\Lambda^{-2}\right)$. As $m_{b} \neq 0$ is retained, the $Z \rightarrow \bar{b} b$ result contains additional terms. The partial width expression in the SM is

$$
\Gamma_{Z \rightarrow \bar{b} b}^{\mathrm{SM}}=\frac{G_{F} m_{Z}^{3}}{\sqrt{2} \pi}\left[g_{d L}^{2}+g_{d R}^{2}\right] \sqrt{1-4 x_{b}^{2}}\left[1-x_{b}^{2}\left(1-\frac{6 g_{d L} g_{d R}}{g_{d L}^{2}+g_{d R}^{2}}\right)\right]
$$


with $x_{b}=m_{b} / m_{Z}$. The relative SMEFT correction is

$$
\begin{aligned}
\frac{\delta \Gamma_{Z \rightarrow \bar{b} b}}{\Gamma_{Z \rightarrow \bar{b} b}^{\mathrm{SM}}}= & \left.\frac{\delta \Gamma_{Z \rightarrow \bar{b} b}}{\Gamma_{Z \rightarrow \bar{b} b}^{\mathrm{SM}}}\right|_{m_{b}=0}+\frac{3 x_{b}^{2}}{\left(g_{d L}^{2}+g_{d R}^{2}\right)\left(1-x_{b}^{2}\right)+6 x_{b}^{2} g_{d L} g_{d R}} \frac{g_{d L}^{2}-g_{d R}^{2}}{g_{d L}^{2}+g_{d R}^{2}} \times \\
& \times\left[-\frac{1}{3} s_{2 \hat{\theta}}^{2}\left(g_{d L}-g_{d R}\right)\left(\frac{\delta g_{W}}{\hat{g}_{W}}-\frac{\delta g_{1}}{\hat{g}_{1}}-\frac{c_{2 \hat{\theta}}}{s_{2 \hat{\theta}}} \bar{C}_{H W B}\right)+g_{d L} \Delta_{d, 33}^{R}-g_{d R} \Delta_{d, 33}^{L}\right] \\
& -\frac{6 \sqrt{2} x_{b}\left(g_{d L}^{2}-g_{d R}^{2}\right)}{\left(g_{d L}^{2}+g_{d R}^{2}\right)\left(1-x_{b}^{2}\right)+6 x_{b}^{2} g_{d L} g_{d R}}\left[c_{\hat{\theta}}\left(\bar{C}_{d W}\right)_{33}+s_{\hat{\theta}}\left(\bar{C}_{d B}\right)_{33}\right]
\end{aligned}
$$

where the first term stands for the contributions in eq. (A.10). The relative SMEFT correction to the total decay width is finally obtained as

$$
\frac{\delta \Gamma_{Z}}{\Gamma_{Z}^{\mathrm{SM}}}=\sum_{f}\left[\frac{\delta \Gamma_{Z \rightarrow f}}{\Gamma_{Z \rightarrow f}^{\mathrm{SM}}}\right] \operatorname{Br}_{Z \rightarrow f}^{\mathrm{SM}},
$$

with $f$ running over all the allowed fermion pairs and $\mathrm{Br}_{Z \rightarrow f}^{\mathrm{SM}}, \Gamma_{Z}^{\mathrm{SM}}$ computed directly from the tree level expressions.

\section{A.2 W boson}

At tree level in the SM, the partial decay width of the $W^{+}$boson into a fermion pair $f^{+}=\left\{e_{p}^{+} \nu_{p}, \bar{d}_{p} u_{p}\right\}$ with flavor $p$ is

$$
\Gamma_{W^{+} \rightarrow f^{+}}^{\mathrm{SM}}=\frac{G_{F} m_{W}^{3}}{6 \sqrt{2} \pi} N_{C}^{f},
$$

with $N_{C}^{f}=\{1,3\}$ the number of colors. Only decays into same-generation fermions are considered here, as CKM mixing is neglected. The relative SMEFT correction for each channel is

$$
\begin{aligned}
& \frac{\delta \Gamma_{W^{+} \rightarrow l_{p}^{+} \nu_{p}}}{\Gamma_{W^{+} \rightarrow l_{p}^{+} \nu_{p}}^{\mathrm{SM}}}=2 \frac{\delta g_{W}}{\hat{g}_{W}}+\frac{\delta m_{W}}{\hat{m}_{W}}+2\left(\bar{C}_{H l}^{(3)}\right)_{p p}, \\
& \frac{\delta \Gamma_{W^{+} \rightarrow u_{p} \bar{d}_{p}}}{\Gamma_{W^{+} \rightarrow u_{p} \bar{d}_{p}}^{\mathrm{SM}}}=2 \frac{\delta g_{W}}{\hat{g}_{W}}+\frac{\delta m_{W}}{\hat{m}_{W}}+2\left(\bar{C}_{H q}^{(3)}\right)_{p p} .
\end{aligned}
$$

The total $W^{+}$decay width in the $\mathrm{SM}$ is

$$
\Gamma_{W}=\sum_{p=1}^{3} \Gamma_{W^{+} \rightarrow l_{p}^{+} \nu_{p}}+\sum_{p=1}^{2} \Gamma_{W^{+} \rightarrow u_{p} \bar{d}_{p}} .
$$

Since in this case the branching ratios are simple rational numbers, the relative SMEFT correction simplifies into

$$
\begin{aligned}
\frac{\delta \Gamma_{W}}{\Gamma_{W}^{\mathrm{SM}}} & =\frac{1}{9} \sum_{p=1}^{3} \frac{\delta \Gamma_{W^{+} \rightarrow l_{p}^{+} \nu_{p}}}{\Gamma_{W^{+} \rightarrow l_{p}^{+} \nu_{p}}^{\mathrm{SM}}}+\frac{1}{3} \sum_{p=1}^{2} \frac{\delta \Gamma_{W^{+} \rightarrow \bar{d}_{p} u_{p}}}{\Gamma_{W^{+} \rightarrow \bar{d}_{p} u_{p}}^{\mathrm{SM}}} \\
& =2 \frac{\delta g_{W}}{\hat{g}_{W}}+\frac{\delta m_{W}}{\hat{m}_{W}}+\frac{2}{9} \sum_{p=1}^{3}\left(\bar{C}_{H l}^{(3)}\right)_{p p}+\frac{2}{3} \sum_{p=1}^{2}\left(\bar{C}_{H q}^{(3)}\right)_{p p} .
\end{aligned}
$$




\section{A.3 Higgs boson}

The SM partial widths for two-body Higgs decays are:

$$
\begin{aligned}
\Gamma_{h \rightarrow \bar{\psi}_{p} \psi_{p}}^{\mathrm{SM}} & =\frac{m_{h} y_{\psi_{p}}^{2}}{16 \pi} N_{C}^{\psi}\left[1-4 x_{\psi_{p}}\right]^{3 / 2}, \\
\Gamma_{h \rightarrow \gamma \gamma}^{\mathrm{SM}} & =\frac{m_{h}^{3}}{4 \pi \hat{v}^{2}} g_{H \gamma \gamma}^{2}, \\
\Gamma_{h \rightarrow Z \gamma}^{\mathrm{SM}} & =\frac{m_{h}^{3}}{8 \pi \hat{v}^{2}} g_{H Z \gamma}^{2}\left[1-x_{Z}^{2}\right]^{3}, \\
\Gamma_{h \rightarrow g g}^{\mathrm{SM}} & =\frac{m_{h}^{3}}{2 \pi \hat{v}^{2}}\left[2 g_{H g g}^{(1)}-\frac{1}{x_{t}^{2}} g_{H g g}^{(2)}\right]^{2},
\end{aligned}
$$

where $x_{\psi_{p}}=m_{\psi_{p}} / m_{h}$ for a generic fermion $\psi_{p}, x_{Z}=m_{Z} / m_{h}, x_{t}=m_{t} / m_{h}$ and $y_{\psi_{p}} \equiv$ $\left(Y_{\psi}\right)_{p p}$ is the relevant Yukawa coupling. The relative SMEFT corrections are

$$
\begin{aligned}
\frac{\delta \Gamma_{h \rightarrow \bar{\psi}_{p} \psi_{p}}}{\Gamma_{h \rightarrow \bar{\psi}_{p} \psi_{p}}^{\mathrm{SM}}} & =1-\Delta G_{F}+2 \Delta \kappa_{H}-2 \frac{\left(\bar{C}_{\psi H}\right)_{p p}}{y_{f_{p}}}, \\
\frac{\delta \Gamma_{h \rightarrow \gamma \gamma}}{\Gamma_{h \rightarrow \gamma \gamma}^{\mathrm{SM}}} & =\frac{2}{g_{H \gamma \gamma}^{2}}\left[c_{\hat{\theta}}^{2} \bar{C}_{H B}+s_{\hat{\theta}}^{2} \bar{C}_{H W}-s_{\hat{\theta}} c_{\hat{\theta}} \bar{C}_{H W B}\right], \\
\frac{\delta \Gamma_{h \rightarrow Z \gamma}}{\Gamma_{h \rightarrow Z \gamma}^{\mathrm{SM}}} & =\frac{2}{g_{H Z \gamma}^{2}}\left[s_{2 \hat{\theta}}\left(\bar{C}_{H W}-\bar{C}_{H B}\right)-c_{2 \hat{\theta}} \bar{C}_{H W B}\right], \\
\frac{\delta \Gamma_{h \rightarrow g g}}{\Gamma_{h \rightarrow g g}^{\mathrm{SM}}} & =\frac{4 \bar{C}_{H G}}{2 g_{H g g}^{(1)}-g_{H g g}^{(2)} / x_{t}^{2}},
\end{aligned}
$$

with the $h \gamma \gamma, h Z \gamma, h g g$ couplings defined in section 5. The SMEFTsim implementation retains the masses of the tau lepton, charm and bottom quarks in eq. (A.24). Fourbody decays were included neglecting neutral current contributions, CKM mixing and all fermion masses. The analytic expressions were taken from ref. [57] and generalized to all flavor setups.

For each individual decay channel, the partial decay width in the SM is

$$
\Gamma_{h \rightarrow f_{+} f_{-}}^{\mathrm{SM}}=N_{C}^{f_{+}} N_{C}^{f_{-}} \frac{m_{W}^{8} G_{F}^{3}}{m_{h}}\left(4.65 \times 10^{-4}\right),
$$

with $f_{+}=\left\{l_{p}^{+} \nu_{p}, \bar{d}_{p} u_{p}\right\}, f_{-}=\left\{\bar{\nu}_{p} l_{p}^{-}, \bar{u}_{p} d_{p}\right\}$ and $N_{C}^{f_{+}}, N_{C}^{f--}$ the appropriate color multiplicities. The numerical factor comes from the phase space integration, that is performed taking $\hat{m}_{W}=80.387 \mathrm{GeV}$.

Summing over all allowed flavor combinations, the relative SMEFT corrections are:

$$
\begin{aligned}
\frac{\delta \Gamma_{h \rightarrow l^{+} \nu \bar{\nu} l^{-}}}{\Gamma_{h \rightarrow l^{+} \nu \bar{\nu} l^{-}}^{\mathrm{SM}}} & =F_{1}+\left(\frac{4}{3}-0.588\right) \sum_{p=1}^{3}\left(\bar{C}_{H l}^{(3)}\right)_{p p}, \\
\frac{\delta \Gamma_{h \rightarrow \bar{u} d \bar{d} u}}{\Gamma_{h \rightarrow \bar{u} d \bar{d} u}^{\mathrm{SM}}} & =F_{1}+(2-0.882) \sum_{p=1}^{2}\left(\bar{C}_{H q}^{(3)}\right)_{p p}, \\
\frac{\delta \Gamma_{h \rightarrow l^{+} \nu \bar{u} d+\text { h.c. }}}{\Gamma_{h \rightarrow l^{+} \nu \bar{u} d+\text { h.c. }}^{\mathrm{SM}}} & =F_{1}+\left(\frac{2}{3}-0.294\right) \sum_{p=1}^{3}\left(\bar{C}_{H l}^{(3)}\right)_{p p}+(1-0.441) \sum_{p=1}^{2}\left(\bar{C}_{H q}^{(3)}\right)_{p p},
\end{aligned}
$$


with

$$
F_{1}=-\Delta G_{F}+2 \Delta \kappa_{H}+4 \frac{\delta g_{W}}{\hat{g}_{W}}-0.463 \frac{\delta \Gamma_{W}}{\Gamma_{W}^{\mathrm{SM}}}-9.643 \frac{\delta m_{W}}{m_{W}}-1.487 \bar{C}_{H W}
$$

The SMEFT correction to the total Higgs width is finally estimated as

$$
\frac{\delta \Gamma_{h}}{\Gamma_{f}^{S M}}=\sum_{f} \operatorname{Br}_{h \rightarrow f}^{S M, \text { best }}\left[\frac{\delta \Gamma_{h \rightarrow f}}{\Gamma_{h \rightarrow f}^{S M}}\right],
$$

with $f=\left\{\bar{b} b, \bar{c} c, \tau^{+} \tau^{-}, \gamma \gamma, Z \gamma, g g, l^{+} \nu \bar{\nu} l^{-}, \bar{u} d \bar{d} u, l^{+} \nu \bar{u} d+\right.$ h.c. $\}$ and the branching ratio values in table 6 .

\section{A.4 Top quark}

To a very good approximation, the top quark decays exclusively to $W b$. The SM width is

$$
\Gamma_{t}^{S M}=\Gamma_{t \rightarrow W^{+} b}^{S M}=\frac{G_{F} m_{t}^{3}}{8 \sqrt{2} \pi}\left[\left(1-x_{b}^{2}\right)^{2}+\left(1+x_{b}^{2}\right) x_{W}^{2}-2 x_{W}^{4}\right] \sqrt{\lambda\left(1, x_{b}^{2}, x_{W}^{2}\right)}
$$

with $x_{b}=m_{b} / m_{t}, x_{W}=\hat{m}_{W} / m_{t}$ and $\lambda(a, b, c)=a^{2}+b^{2}+c^{2}-2 a b-2 a c-2 b c$. The relative SMEFT correction is:

$$
\begin{aligned}
\frac{\delta \Gamma_{t}}{\Gamma_{t}^{S M}}= & \frac{\delta \Gamma_{t \rightarrow W^{+} b}}{\Gamma_{t \rightarrow W+b}^{\mathrm{SM}}} \\
= & 2 \frac{\delta g_{W}}{\hat{g}_{W}}+2\left(\bar{C}_{H q}^{(3)}\right)_{33}+\frac{6 x_{W}}{\left(1-x_{b}^{2}\right)^{2}+\left(1+x_{b}^{2}\right) x_{W}^{2}-2 x_{W}^{4}}\left[x_{W} x_{b}\left(\operatorname{Re} \bar{C}_{H u d}\right)_{33}+\right. \\
& \left.-\sqrt{2}\left(\operatorname{Re} \bar{C}_{u W}\right)_{33}\left(1-x_{W}^{2}-x_{b}^{2}\right)+\sqrt{2}\left(\operatorname{Re} \bar{C}_{d W}\right)_{33} x_{b}\left(1+x_{W}^{2}-x_{b}^{2}\right)\right]+ \\
& +2\left[\frac{\left(1-x_{b}^{2}\right)^{2}-3\left(1+x_{b}^{2}\right) x_{W}^{2}+2 x_{W}^{4}}{\lambda\left(1, x_{b}^{2}, x_{W}^{2}\right)}-\frac{2\left(1-x_{b}^{2}\right)^{2}+\left(1+x_{b}\right)^{2} x_{W}^{2}}{\left(1-x_{b}^{2}\right)^{2}+\left(1+x_{b}^{2}\right) x_{W}^{2}-2 x_{W}^{4}}\right] \frac{\delta m_{W}}{\hat{m}_{W}} .
\end{aligned}
$$

Decaying the $W$ in final state does not lead to any additional contribution to $\delta \Gamma_{t} / \Gamma_{t}^{\mathrm{SM}}$. This happens because the $W$ is always on-shell, so its decay essentially factorizes out: using the narrow width approximation, one trivially has

$$
\Gamma_{t}=\sum_{f} \Gamma_{t \rightarrow W^{+} b} \cdot \mathrm{Br}_{W^{+} \rightarrow f}=\Gamma_{t \rightarrow W^{+} b} .
$$

Note that this conclusion only holds at the $\mathrm{SM}-\mathcal{L}_{6}$ interference level, while at $\mathcal{O}\left(\Lambda^{-4}\right)$ additional SMEFT corrections arise through contact vertices $(\bar{t} b)(\bar{\nu} l),(\bar{t} b)(\bar{d} u)$. 


\section{B What's new in version 3.0}

Here we briefly summarize the most significant updates and features introduced in SMEFTsim 3.0, compared to previous versions:

- The flavor assumptions top and topU31 described in section 3.4 have been added.

- The flavor structure of all models has been generally improved. See section 3.5 for details.

- The treatment of propagator corrections described in section 6 has been implemented, enabling the estimate of linearized EFT corrections to the $W, Z, H, t$ widths and to the $W$ mass.

- The treatment of SM loop-generated Higgs interactions has been improved, particularly in the Higgs-gluon case. See section 5 for the general treatment and section 5.2 for a detailed comparison with previous implementations.

- All interaction vertices with up to 6 legs are now included in all UFO models. In the previous version, only 4-point functions were retained.

- The numerical values of the SM parameters have been updated, see table 16. The default value has been set to 0 for all Wilson coefficients.

- All complex Wilson coefficients are expressed in terms of their real and imaginary parts, rather than absolute values and phases.

- Individual interaction orders have been defined for each operator. Additionally, interaction orders NPcpv, NPprop, NPfv have been added, to provide more control on each class of EFT contributions. See section 8.2 for further details.

- In the UFO models, the SMEFT parameters have been organized in parameters blocks: SMEFTcutoff contains only $\Lambda$ and SMEFT (SMEFTcpv) contain CP conserving (violating) Wilson coefficients. In the MFV models the flavor-violating $\Delta C_{\alpha}$ parameters are contained in the additional SMEFTFV block. See section 8.1.

- The normalization of $\Delta G_{F}$ (dGf) has been modified. This is explicit in the FeynRules Lagrangian but does not have any consequence for the UFO models.

\section{Conversion tables between flavor assumptions}

This appendix collects the results of section 3 and compares the flavor structure of the fermionic operators across the five setups considered. Tables 10-15 provide a dictionary between the different models: in order to translate between two flavor assumptions it is sufficient to exchange the corresponding expressions within each table block. All structures are given explicitly in terms of diagonal Yukawa matrices and of the CKM matrix $V$. In the top and topU3l cases, $Y_{u}$ and $Y_{d}$ are $2 \times 2$ matrices and $V=\mathbb{1}$ is assumed. 


\begin{tabular}{|c|c|}
\hline $\begin{array}{r}\text { general } \\
\text { U35, MFV, topU31 } \\
\text { top }\end{array}$ & $\begin{array}{l}\left(C_{e H}\right)_{p r} \\
C_{e H}\left(Y_{l}^{(d)}\right)_{p r} \\
\left(C_{e H}\right)_{p p} \delta_{p r}\end{array}$ \\
\hline general & $\left(C_{u H}\right)_{p r}$ \\
\hline U35 & $C_{u H}\left(Y_{u}^{(d)}\right)_{p r}$ \\
\hline MFV & {$\left[C_{u H}^{(0)} Y_{u}^{(d)}+\left(\Delta^{u} C_{u H}\right)\left(Y_{u}^{(d)}\right)^{3}+\left(\Delta^{d} C_{u H}\right) V\left(Y_{d}^{(d)}\right)^{2} V^{\dagger} Y_{u}^{(d)}\right]_{p r}$} \\
\hline top, topU31 & $C_{u H}\left(Y_{u}^{(d)}\right)_{p r} \quad p, r=\{1,2\}, \quad C_{t H} \quad p=r=3$ \\
\hline general & $\left(V^{\dagger} C_{d H}\right)_{p r}$ \\
\hline U35 & $C_{d H}\left(Y_{d}^{(d)}\right)_{p r}$ \\
\hline $\mathrm{MFV}$ & {$\left[C_{d H}^{(0)} Y_{d}^{(d)}+\left(\Delta^{u} C_{d H}\right) V^{\dagger}\left(Y_{u}^{(d)}\right)^{2} V Y_{d}^{(d)}+\left(\Delta^{d} C_{d H}\right)\left(Y_{d}^{(d)}\right)^{3}\right]_{p r}$} \\
\hline top, topU31 & $C_{d H}\left(Y_{d}^{(d)}\right)_{p r} \quad p, r=\{1,2\}, \quad C_{b H} \quad p=r=3$ \\
\hline general & Same as $\left(C_{e H}\right)_{p r}$. \\
\hline general & Same as $\left(C_{e H}\right)_{p r}$. \\
\hline general & Same as $\left(C_{u H}\right)_{p r}$. \\
\hline general & Same as $\left(C_{u H}\right)_{p r}$. \\
\hline general & Same as $\left(C_{u H}\right)_{p r}$. \\
\hline general & Same as $\left(V^{\dagger} C_{d H}\right)_{p r}$ \\
\hline general & Same as $\left(V^{\dagger} C_{d H}\right)_{p r}$ \\
\hline general & Same as $\left(V^{\dagger} C_{d H}\right)_{p r}$ \\
\hline
\end{tabular}

Table 10. Conversion table among the 5 flavor assumptions considered for the operators in $\mathcal{L}_{6}^{(5)}, \mathcal{L}_{6}^{(6)}$. 


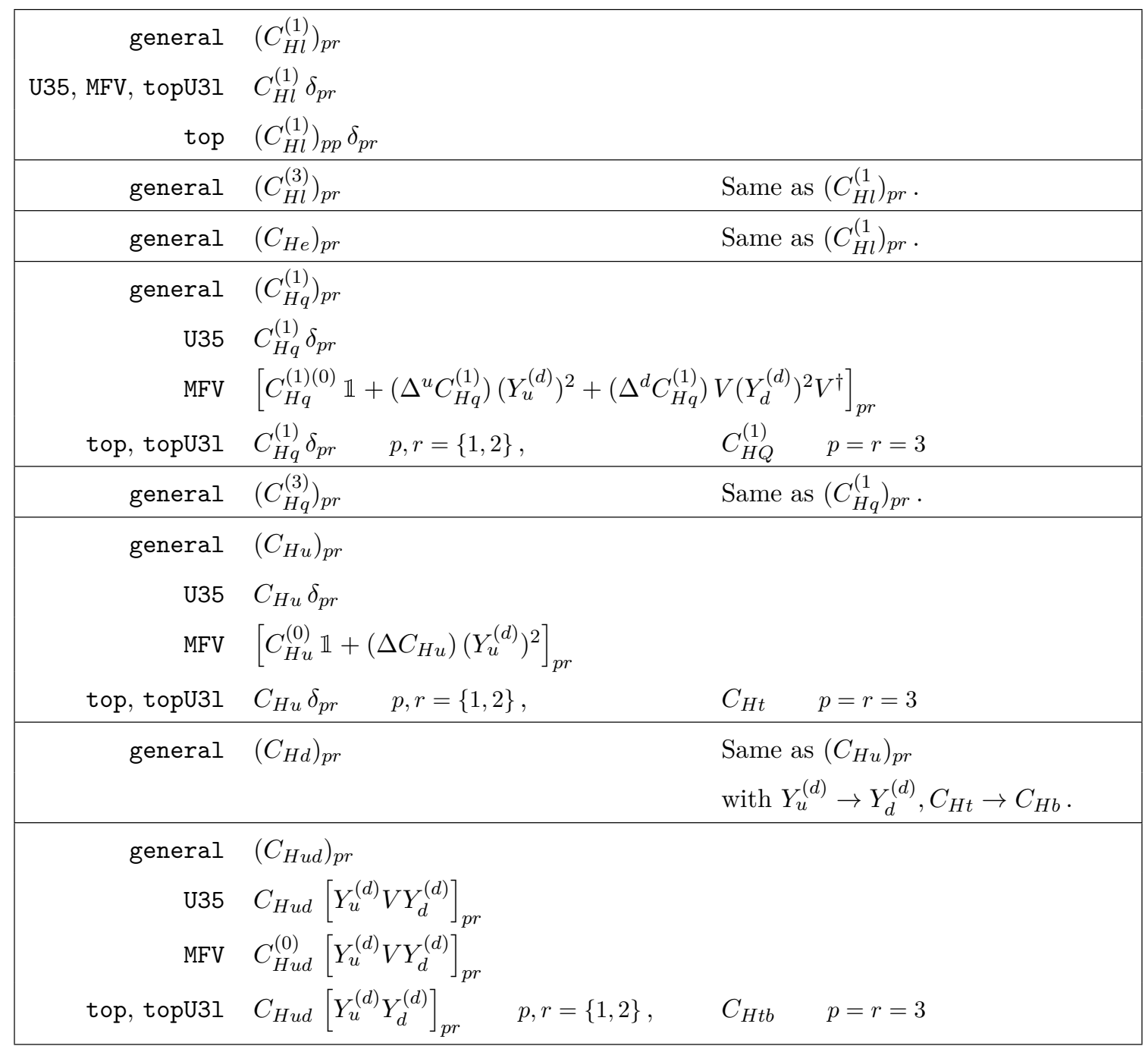

Table 11. Conversion table among the 5 flavor assumptions considered, for the operators in $\mathcal{L}_{6}^{(7)}$. 


\begin{tabular}{|c|c|}
\hline $\begin{array}{r}\text { general } \\
\text { U35, MFV, topU31 } \\
\text { top }\end{array}$ & $\begin{array}{l}\left(C_{l l}\right)_{p r s t} \\
C_{l l} \delta_{p r} \delta_{s t}+C_{l l}^{\prime} \delta_{p t} \delta_{s r} \\
\frac{1}{2}\left(C_{l l}\right)_{p r s t}\left(\delta_{p r} \delta_{s t}+\delta_{p t} \delta_{s r}\right), \quad \text { prst } \in P_{l l}\end{array}$ \\
\hline $\begin{array}{r}\text { general } \\
\text { U35 } \\
\text { MFV }\end{array}$ & 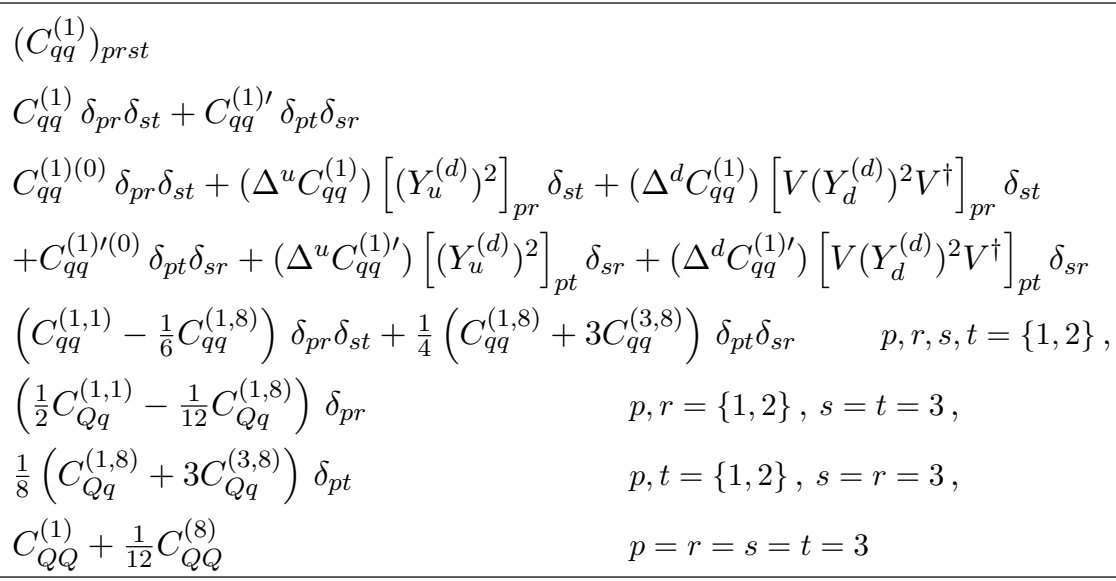 \\
\hline $\begin{array}{r}\text { general } \\
\text { U35 }\end{array}$ & $\begin{array}{l}\left(C_{q q}^{(3)}\right)_{p r s t} \\
C_{q q}^{(3)} \delta_{p r} \delta_{s t}+C_{q q}^{(3) \prime} \delta_{p t} \delta_{s r} \\
C_{q q}^{(3)(0)} \delta_{p r} \delta_{s t}+\left(\Delta^{u} C_{q q}^{(3)}\right)\left[\left(Y_{u}^{(d)}\right)^{2}\right]_{p r} \delta_{s t}+\left(\Delta^{d} C_{q q}^{(3)}\right)\left[V\left(Y_{d}^{(d)}\right)^{2} V^{\dagger}\right]_{p r} \delta_{s t} \\
+C_{q q}^{(3) \prime(0)} \delta_{p t} \delta_{s r}+\left(\Delta^{u} C_{q q}^{(3) \prime}\right)\left[\left(Y_{u}^{(d)}\right)^{2}\right]_{p t} \delta_{s r}+\left(\Delta^{d} C_{q q}^{(3) \prime}\right)\left[V\left(Y_{d}^{(d)}\right)^{2} V^{\dagger}\right]_{p t} \delta_{s r} \\
\left(C_{q q}^{(3,1)}-\frac{1}{6} C_{q q}^{(3,8)}\right) \delta_{p r} \delta_{s t}+\frac{1}{4}\left(C_{q q}^{(1,8)}-C_{q q}^{(3,8)}\right) \delta_{p t} \delta_{s r} \quad p, r, s, t=\{1,2\}, \\
\left(\frac{1}{2} C_{Q q}^{(3,1)}-\frac{1}{12} C_{Q q}^{(3,8)}\right) \delta_{p r} \\
\begin{array}{l}\frac{1}{8}\left(C_{Q q}^{(1,8)}-C_{Q q}^{(3,8)}\right) \delta_{p t} \\
\frac{1}{4} C_{Q Q}^{(8)}\end{array} \quad p, r=\{1,2\}, s=t=3, \\
p=r 1,2\}, s=r=3,\end{array}$ \\
\hline $\begin{array}{r}\text { general } \\
\text { U35 }\end{array}$ & 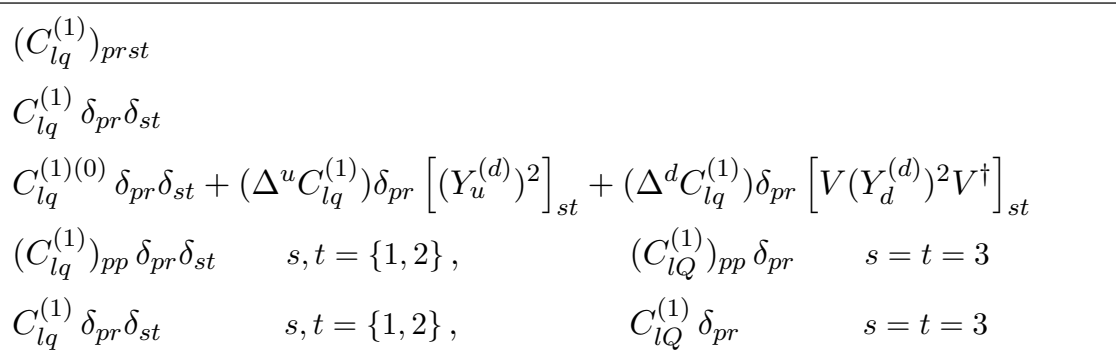 \\
\hline general & Same as $\left(C_{l q}^{(1)}\right)_{p r s t}$ \\
\hline
\end{tabular}

Table 12. Conversion table among the 5 flavor assumptions considered, for the operators in $\mathcal{L}_{6}^{(8 a)}$. The set $P_{l l}$ is defined in eq. (3.70). 


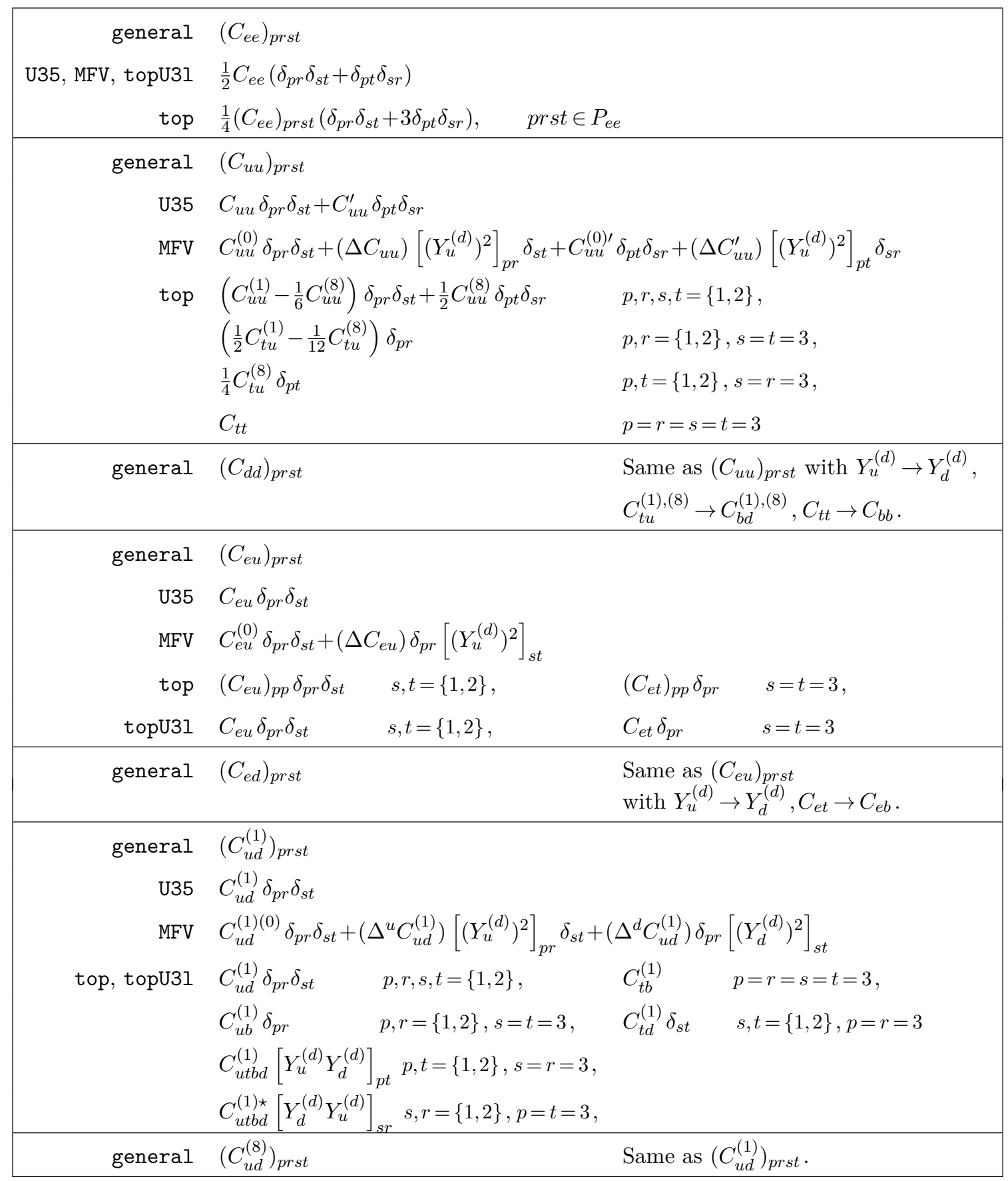

Table 13. Conversion table among the 5 flavor assumptions considered, for the operators in $\mathcal{L}_{6}^{(8 b)}$. The set $P_{e e}$ is defined in eq. (3.91). 


\begin{tabular}{|c|c|}
\hline $\begin{array}{r}\text { general } \\
\text { U35, MFV, topU31 } \\
\text { top }\end{array}$ & $\begin{array}{l}\left(C_{l e}\right)_{p r s t} \\
C_{l e} \delta_{p r} \delta_{s t} \\
\left(C_{l e}\right)_{p r s t}\end{array}$ \\
\hline $\begin{array}{r}\text { top } \\
\text { topU31 }\end{array}$ & $\begin{array}{l}\left(C_{l u}\right)_{p r s t} \\
C_{l u} \delta_{p r} \delta_{s t} \\
C_{l u}^{(0)} \delta_{p r} \delta_{s t}+\left(\Delta C_{l u}\right) \delta_{p r}\left[\left(Y_{u}^{(d)}\right)^{2}\right]_{s t} \\
\left(C_{l u}\right)_{p p} \delta_{p r} \delta_{s t} \quad s, t=\{1,2\} \\
C_{l u} \delta_{p r} \delta_{s t} \quad s, t=\{1,2\}\end{array}$ \\
\hline general & $\begin{array}{l}\text { Same as }\left(C_{l u}\right)_{p r s t} \\
\text { with } Y_{u}^{(d)} \rightarrow Y_{d}^{(d)}, C_{l t} \rightarrow C_{l b}\end{array}$ \\
\hline $\begin{array}{r}\text { MFV } \\
\text { top } \\
\text { topU31 }\end{array}$ & 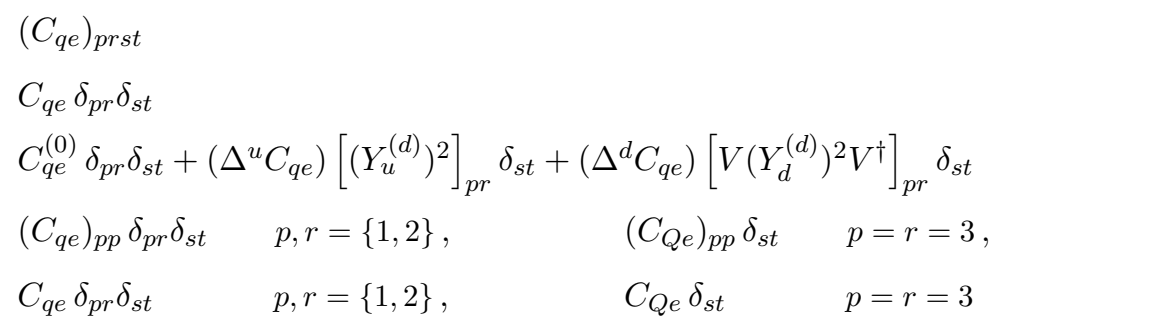 \\
\hline $\begin{array}{r}\text { general } \\
\text { U35 } \\
\text { MFV }\end{array}$ & 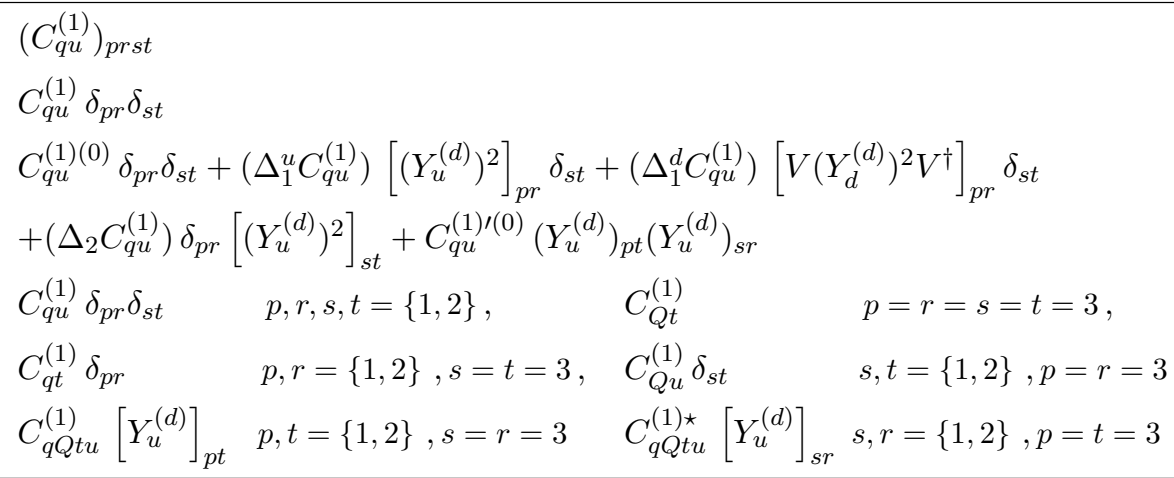 \\
\hline general & Same as $\left(C_{q u}^{(1)}\right)_{p r s t}$ \\
\hline $\begin{array}{r}\text { general } \\
\text { U35 }\end{array}$ & 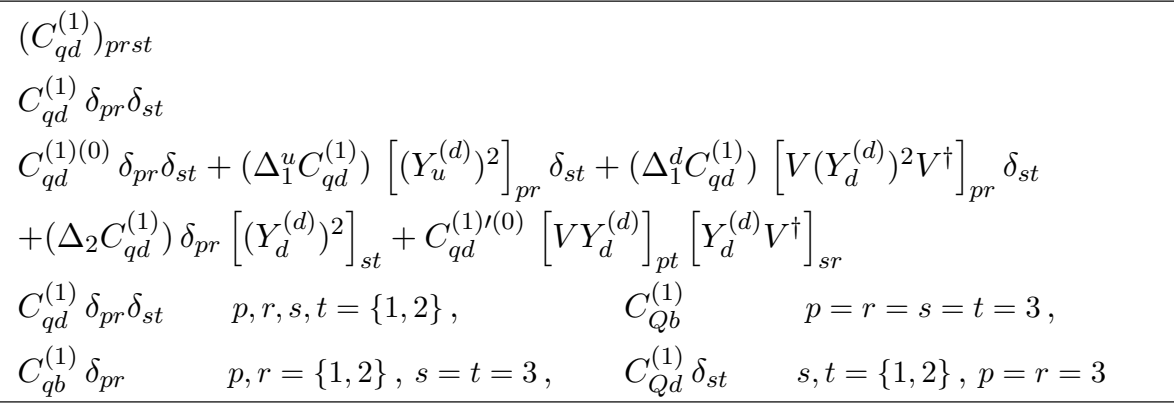 \\
\hline general & Same as $\left(C_{q d}^{(1)}\right)_{p r s t}$ \\
\hline
\end{tabular}

Table 14. Conversion table among the 5 flavor assumptions considered, for the operators in $\mathcal{L}_{6}^{(8 c)}$. The set $P_{l e}$ is defined in eq. (3.93). 


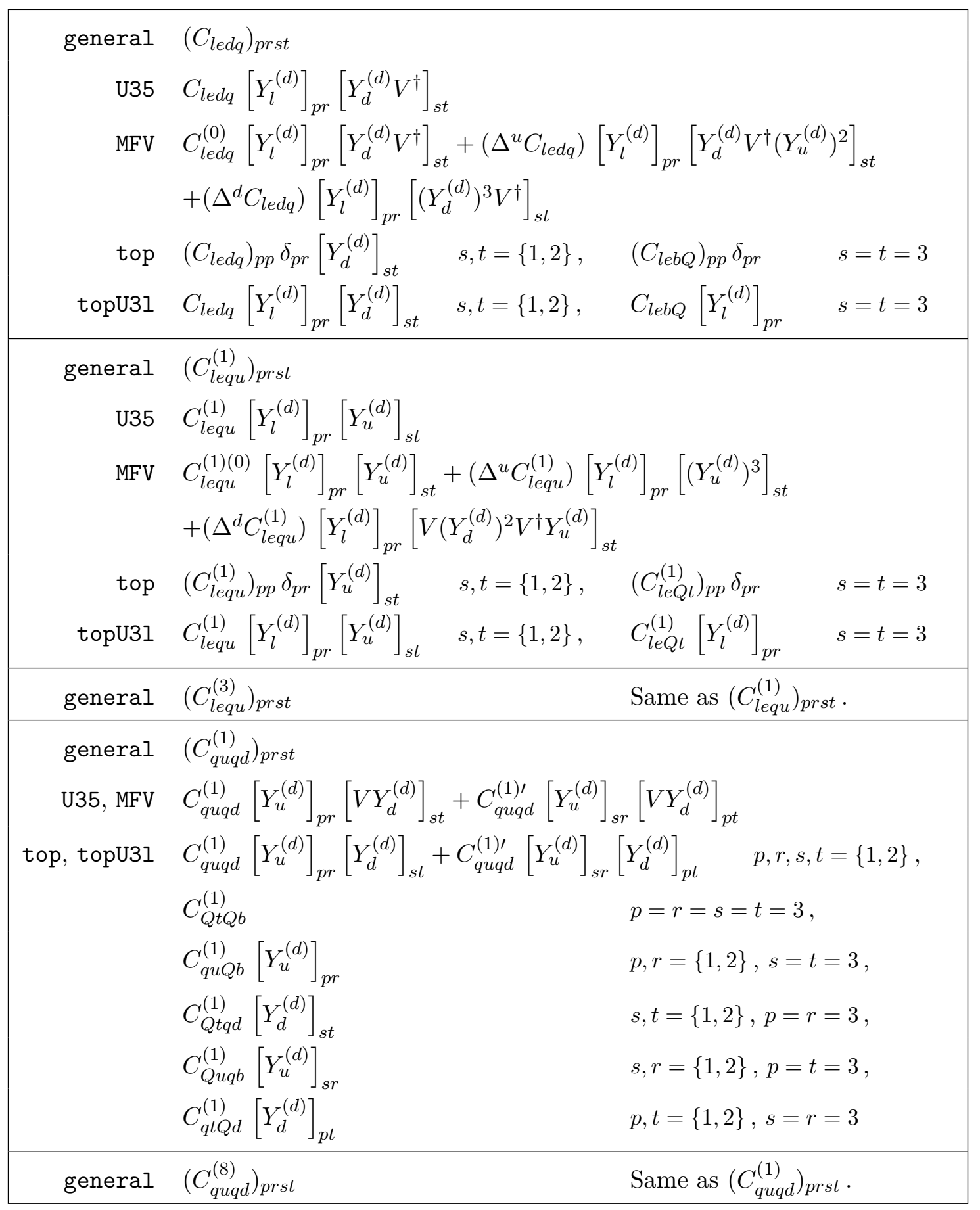

Table 15. Conversion table among the 5 flavor assumptions considered, for the operators in $\mathcal{L}_{6}^{(8 d)}$. 


\section{Parameter definitions in the code implementation}

This appendix provides tables to facilitate the interpretation of the FeynRules and UFO implementations in terms of the theory discussion in the main text.

Table 16 lists the external parameters that are defined in all SMEFTsim models, specifying the corresponding code name and default numerical value. Table 17 shows the nomenclature used for the Wilson coefficients of the bosonic operators and for the shift quantities defined in section 4.1. Tables 18-23 do the same for the Wilson coefficients of fermionic operators, for each flavor assumption. As a common rationale, primes are replaced by 1 in the code name and real and imaginary parts are specified by with Re, Im suffixes. If needed, flavor indices are fully specified and appended at the very end of the code names. In the MFV models, the coefficients $\Delta_{n}^{q}\left(C_{\alpha}\right)$ are denoted Delta[n] [q] c [a].

Although the correspondence between parameters names is most often direct, some notational changes were necessary, particularly in the top and topU31 implementations. Most notably the lowercase $q$ has been replaced with $j$ in all the parameters' and operators' names, as the $q / Q$ distinction between light and heavy quark fields is problematic for noncase-sensitive interfaces. Analogously, the coefficient $C_{H b}$ is denoted as cHbq to avoid conflict with $\mathrm{cHB}$, while $C_{b B}$ is denoted as $\mathrm{cbBB}$, distinct from cbb. The internal parameter $C_{t H}$ is denoted as ctHH to avoid conflict with the cosine of the weak angle cth. 


\begin{tabular}{|c|c|c|c|c|c|c|c|}
\hline \multicolumn{8}{|c|}{ Common parameters defined in SMEFTsim } \\
\hline parameter & UFO & default value & & parameter & UFO & default value & \\
\hline$G_{F}$ & Gf & $1.1663787 \times 10^{-5} \mathrm{GeV}^{-2}$ & {$[82,118]$} & $\alpha_{s}\left(m_{Z}\right)$ & aS & 0.1179 & {$[82]$} \\
\hline$\alpha_{\mathrm{em}}\left(m_{Z}\right)\left(^{\star}\right)$ & $\mathrm{aEW}$ & $1 / 127.95$ & {$[82,118]$} & $m_{W}(\star \star)$ & MW & $80.387 \mathrm{GeV}$ & [119] \\
\hline$m_{d}$ & MD & $4.67 \times 10^{-3} \mathrm{GeV}$ & {$[82]$} & $y_{d} \hat{v} / \sqrt{2}$ & ymdo & $4.67 \times 10^{-3} \mathrm{GeV}$ & {$[82]$} \\
\hline$m_{s}$ & MS & $0.093 \mathrm{GeV}$ & {$[82]$} & $y_{s} \hat{v} / \sqrt{2}$ & yms & $0.093 \mathrm{GeV}$ & {$[82]$} \\
\hline$m_{b}$ & MB & $4.18 \mathrm{GeV}$ & {$[82]$} & $y_{b} \hat{v} / \sqrt{2}$ & ymb & $4.18 \mathrm{GeV}$ & {$[82]$} \\
\hline$m_{u}$ & MU & $2.16 \times 10^{-3} \mathrm{GeV}$ & {$[82]$} & $y_{u} \hat{v} / \sqrt{2}$ & ymup & $2.16 \times 10^{-3} \mathrm{GeV}$ & {$[82]$} \\
\hline$m_{c}$ & MC & $1.27 \mathrm{GeV}$ & {$[82]$} & $y_{c} \hat{v} / \sqrt{2}$ & ymc & $1.27 \mathrm{GeV}$ & {$[82]$} \\
\hline$m_{t}$ & MT & $172.76 \mathrm{GeV}$ & {$[82]$} & $y_{t} \hat{v} / \sqrt{2}$ & ymt & $172.76 \mathrm{GeV}$ & {$[82]$} \\
\hline$m_{e}$ & $\mathrm{Me}$ & $5.11 \times 10^{-4} \mathrm{GeV}$ & {$[82]$} & $y_{e} \hat{v} / \sqrt{2}$ & yme & $5.11 \times 10^{-4} \mathrm{GeV}$ & {$[82]$} \\
\hline$m_{\mu}$ & MMU & $0.10566 \mathrm{GeV}$ & {$[82]$} & $y_{\mu} \hat{v} / \sqrt{2}$ & $\mathrm{ymm}$ & $0.10566 \mathrm{GeV}$ & {$[82]$} \\
\hline$m_{\tau}$ & MTA & $1.777 \mathrm{GeV}$ & {$[82]$} & $y_{\tau} \hat{v} / \sqrt{2}$ & ymtau & $1.777 \mathrm{GeV}$ & {$[82]$} \\
\hline$m_{Z}$ & MZ & $91.1876 \mathrm{GeV}$ & {$[82,118,120]$} & $m_{h}$ & MH & $125.09 \mathrm{GeV}$ & {$[82]$} \\
\hline$\Gamma_{Z}^{S M, \text { best }}$ & WZ & $2.4952 \mathrm{GeV}$ & {$[82]$} & $\Gamma_{h}^{S M, \text { best }}$ & WH & $4.07 \times 10^{-3} \mathrm{GeV}$ & {$[113]$} \\
\hline$\Gamma_{W}^{S M, \text { best }}$ & WW & $2.085 \mathrm{GeV}$ & {$[82]$} & $\Gamma_{t}^{S M, \text { best }}$ & WT & $1.33 \mathrm{GeV}$ & {$[105]$} \\
\hline$\lambda_{C K M}$ & CKMlambda & 0.22650 & {$[82]$} & $A$ & CKMA & 0.790 & {$[82]$} \\
\hline$\rho$ & CKMrho & 0.141 & {$[82]$} & $\eta$ & CKMeta & 0.357 & {$[82]$} \\
\hline$\Lambda$ & LambdaSMEFT & $1 \mathrm{TeV}$ & & & & & \\
\hline
\end{tabular}

$\left({ }^{*}\right)$ only in models with $\left\{\alpha_{\mathrm{em}}, m_{Z}, G_{F}\right\}$ inputs. $\left.{ }^{\star \star}\right)$ only in models with $\left\{m_{W}, m_{Z}, G_{F}\right\}$ inputs.

Table 16. Common external parameters defined in all models, with the corresponding name in the code and default numerical value. The latter have been updated compared to previous SMEFTsim versions, according to the references indicated.

\begin{tabular}{|l|ll|ll|ll|ll|}
\hline \multicolumn{7}{|c|}{ Bosonic SMEFT parameters in SMEFTsim } \\
\hline $\mathcal{L}_{6}^{(1)}$ & $C_{G}$ & $\mathrm{cG}$ & $C_{\widetilde{G}}$ & cGtil & $C_{W}$ & $\mathrm{cW}$ & $C_{\widetilde{W}}$ & cWtil \\
\hline $\mathcal{L}_{6}^{(2,3)}$ & $C_{H}$ & $\mathrm{cH}$ & $C_{H \square}$ & cHbox & $C_{H D}$ & cHDD & & \\
\hline \multirow{2}{*}{$\mathcal{L}_{6}^{(4)}$} & $C_{H G}$ & $\mathrm{cHG}$ & $C_{H W}$ & $\mathrm{cHW}$ & $C_{H B}$ & $\mathrm{cHB}$ & $C_{H W B}$ & cHWB \\
& $C_{H \widetilde{G}}$ & cHGtil & $C_{H \widetilde{W}}$ & cHWtil & $C_{H \widetilde{B}}$ & cHBtil & $C_{H \widetilde{W} B}$ & cHWBtil \\
\hline
\end{tabular}

\begin{tabular}{|ll|ll|ll|ll|}
\hline \multicolumn{8}{|c|}{ Shift parameters in SMEFTsim } \\
\hline$\Delta m_{Z}^{2}$ & $\mathrm{dMZ2}$ & $\Delta m_{h}^{2}$ & $\mathrm{dMH} 2$ & $\Delta G_{F}$ & $\mathrm{dGf}$ & $\Delta \kappa_{H}$ & $\mathrm{dkH}$ \\
\hline$\delta \Gamma_{Z}$ & $\mathrm{dWZ}$ & $\delta \Gamma_{W}$ & $\mathrm{dWW}$ & $\delta \Gamma_{h}$ & $\mathrm{dWH}$ & $\delta \Gamma_{t}$ & $\mathrm{dWT}$ \\
\hline$\delta g_{1} / \hat{g}_{1}$ & $\mathrm{dg} 1$ & $\delta g_{W} / \hat{g}_{W}$ & $\mathrm{dgW}$ & $\delta m_{W}$ & $\mathrm{dMW}$ & & \\
\hline
\end{tabular}

Table 17. Upper block: Wilson coefficients for the 15 bosonic operators. They are common to all flavor versions except MFV, where the CP violating $C_{\widetilde{G}}, C_{\widetilde{W}}, C_{H \widetilde{G}}, C_{H \widetilde{W}}, C_{H \widetilde{B}}, C_{H \widetilde{W} B}$ are not defined. Lower block: shift parameters defined in SMEFTsim, see section 4.1 for details. $\delta m_{W}$ is defined only in models with the $\left\{\alpha_{\mathrm{em}}, m_{Z}, G_{F}\right\}$ input scheme. 


\begin{tabular}{|c|c|c|c|c|c|c|}
\hline \multicolumn{7}{|c|}{ Fermionic SMEFT parameters in SMEFTsim general } \\
\hline class & parameter & UFO & {$[\mathrm{pr}(\mathrm{st})]$} & parameter & UFO & {$[p r(s t)]$} \\
\hline \multirow{3}{*}{$\mathcal{L}_{6}^{(5)}$} & $\operatorname{Re}\left(C_{e H}\right)_{p r}$ & ceHRe $[\mathrm{pr}]$ & {$[2 \mathrm{f}-\mathrm{NH}-\mathrm{R}]$} & $\operatorname{Im}\left(C_{e H}\right)_{p r}$ & ceHIm $[\mathrm{pr}]$ & {$[2 \mathrm{f}-\mathrm{NH}-\mathrm{I}]$} \\
\hline & $\operatorname{Re}\left(C_{u H}\right)_{p r}$ & cdHRe $[\mathrm{pr}]$ & {$[2 \mathrm{f}-\mathrm{NH}-\mathrm{R}]$} & $\operatorname{Im}\left(C_{u H}\right)_{p r}$ & $\mathrm{cdHIm}[\mathrm{pr}]$ & {$[2 \mathrm{f}-\mathrm{NH}-\mathrm{I}]$} \\
\hline & $\operatorname{Re}\left(C_{d H}\right)_{p r}$ & cdHRe $[\mathrm{pr}]$ & {$[2 \mathrm{f}-\mathrm{NH}-\mathrm{R}]$} & $\operatorname{Im}\left(C_{d H}\right)_{p r}$ & $\mathrm{cdHIm}[\mathrm{pr}]$ & {$[2 \mathrm{f}-\mathrm{NH}-\mathrm{I}]$} \\
\hline \multirow{8}{*}{$\mathcal{L}_{6}^{(6)}$} & $\operatorname{Re}\left(C_{e W}\right)_{p r}$ & ceWRe $[\mathrm{pr}]$ & {$[2 \mathrm{f}-\mathrm{NH}-\mathrm{R}]$} & $\operatorname{Im}\left(C_{e W}\right)_{p r}$ & ceWIm $[\mathrm{pr}]$ & {$[2 \mathrm{f}-\mathrm{NH}-\mathrm{I}]$} \\
\hline & $\operatorname{Re}\left(C_{e B}\right)_{p r}$ & ceBRe $[\mathrm{pr}]$ & {$[2 \mathrm{f}-\mathrm{NH}-\mathrm{R}]$} & $\operatorname{Im}\left(C_{e B}\right)_{p r}$ & $\mathrm{ceBIm}[\mathrm{pr}]$ & {$[2 \mathrm{f}-\mathrm{NH}-\mathrm{I}]$} \\
\hline & $\operatorname{Re}\left(C_{u G}\right)_{p r}$ & cuGRe $[\mathrm{pr}]$ & {$[2 \mathrm{f}-\mathrm{NH}-\mathrm{R}]$} & $\operatorname{Im}\left(C_{u G}\right)_{p r}$ & cuGIm [pr] & {$[2 \mathrm{f}-\mathrm{NH}-\mathrm{I}]$} \\
\hline & $\operatorname{Re}\left(C_{u W}\right)_{p r}$ & cuWRe $[\mathrm{pr}]$ & {$[2 \mathrm{f}-\mathrm{NH}-\mathrm{R}]$} & $\operatorname{Im}\left(C_{u W}\right)_{p r}$ & cuWIm $[\mathrm{pr}]$ & {$[2 \mathrm{f}-\mathrm{NH}-\mathrm{I}]$} \\
\hline & $\operatorname{Re}\left(C_{u B}\right)_{p r}$ & cuBRe $[\mathrm{pr}]$ & {$[2 \mathrm{f}-\mathrm{NH}-\mathrm{R}]$} & $\operatorname{Im}\left(C_{u B}\right)_{p r}$ & $\mathrm{cuBIm}[\mathrm{pr}]$ & {$[2 \mathrm{f}-\mathrm{NH}-\mathrm{I}]$} \\
\hline & $\operatorname{Re}\left(C_{d G}\right)_{p r}$ & cdGRe [pr] & {$[2 \mathrm{f}-\mathrm{NH}-\mathrm{R}]$} & $\operatorname{Im}\left(C_{d G}\right)_{p r}$ & $\operatorname{cdGIm}[\mathrm{pr}]$ & {$[2 \mathrm{f}-\mathrm{NH}-\mathrm{I}]$} \\
\hline & $\operatorname{Re}\left(C_{d W}\right)_{p r}$ & cdWRe [pr] & {$[2 \mathrm{f}-\mathrm{NH}-\mathrm{R}]$} & $\operatorname{Im}\left(C_{d W}\right)_{p r}$ & cdWIm [pr] & {$[2 \mathrm{f}-\mathrm{NH}-\mathrm{I}]$} \\
\hline & $\operatorname{Re}\left(C_{d B}\right)_{p r}$ & cdBRe [pr] & {$[2 \mathrm{f}-\mathrm{NH}-\mathrm{R}]$} & $\operatorname{Im}\left(C_{d B}\right)_{p r}$ & $\mathrm{cdBIm}[\mathrm{pr}]$ & [2f-NH-I] \\
\hline \multirow{8}{*}{$\mathcal{L}_{6}^{(7)}$} & $\operatorname{Re}\left(C_{H l}^{(1)}\right)_{p r}$ & cHl1Re [pr] & {$[2 \mathrm{f}-\mathrm{H}-\mathrm{R}]$} & $\operatorname{Im}\left(C_{H l}^{(1)}\right)_{p r}$ & cHl1Im [pr] & {$[2 \mathrm{f}-\mathrm{H}-\mathrm{I}]$} \\
\hline & $\operatorname{Re}\left(C_{H l}^{(3)}\right)_{p r}$ & cH13Re [pr] & {$[2 \mathrm{f}-\mathrm{H}-\mathrm{R}]$} & $\operatorname{Im}\left(C_{H l}^{(3)}\right)_{p r}$ & $\mathrm{cH} 13 \operatorname{Im}[\mathrm{pr}]$ & {$[2 \mathrm{f}-\mathrm{H}-\mathrm{I}]$} \\
\hline & $\operatorname{Re}\left(C_{H e}\right)_{p r}$ & cHeRe $[\mathrm{pr}]$ & {$[2 \mathrm{f}-\mathrm{H}-\mathrm{R}]$} & $\operatorname{Im}\left(C_{H e}\right)_{p r}$ & cHeIm [pr] & {$[2 \mathrm{f}-\mathrm{H}-\mathrm{I}]$} \\
\hline & $\operatorname{Re}\left(C_{H q}^{(1)}\right)_{p r}$ & $\mathrm{cHq} 1 \mathrm{Re}$ [pr] & {$[2 \mathrm{f}-\mathrm{H}-\mathrm{R}]$} & $\operatorname{Im}\left(C_{H q}^{(1)}\right)_{p r}$ & cHq1Im [pr] & {$[2 f-H-I]$} \\
\hline & $\operatorname{Re}\left(C_{H q}^{(3)}\right)_{p r}$ & cHq3Re [pr] & {$[2 \mathrm{f}-\mathrm{H}-\mathrm{R}]$} & $\operatorname{Im}\left(C_{H q}^{(3)}\right)_{p r}$ & cHq3Im [pr] & {$[2 \mathrm{f}-\mathrm{H}-\mathrm{I}]$} \\
\hline & $\operatorname{Re}\left(C_{H u}\right)_{p r}$ & cHuRe $[\mathrm{pr}]$ & {$[2 \mathrm{f}-\mathrm{H}-\mathrm{R}]$} & $\operatorname{Im}\left(C_{H u}\right)_{p r}$ & $\mathrm{cHu} \operatorname{Im}[\mathrm{pr}]$ & {$[2 f-H-I]$} \\
\hline & $\operatorname{Re}\left(C_{H d}\right)_{p r}$ & cHdRe [pr] & {$[2 \mathrm{f}-\mathrm{H}-\mathrm{R}]$} & $\operatorname{Im}\left(C_{H d}\right)_{p r}$ & cHdIm [pr] & {$[2 \mathrm{f}-\mathrm{H}-\mathrm{I}]$} \\
\hline & $\operatorname{Re}\left(C_{H u d}\right)_{p r}$ & cHudRe [pr] & {$[2 \mathrm{f}-\mathrm{NH}-\mathrm{R}]$} & $\operatorname{Im}\left(C_{H u d}\right)_{p r}$ & cHudIm [pr] & {$[2 \mathrm{f}-\mathrm{NH}-\mathrm{I}]$} \\
\hline \multirow{5}{*}{$\mathcal{L}_{6}^{(8 a)}$} & $\operatorname{Re}\left(C_{l l}\right)_{p r s t}$ & cllRe [prst] & {$[4 \mathrm{f}-\mathrm{H}-\mathrm{S}-\mathrm{R}]$} & $\operatorname{Im}\left(C_{l l}\right)_{p r s t}$ & cllIm [prst] & {$[4 \mathrm{f}-\mathrm{H}-\mathrm{S}-\mathrm{I}]$} \\
\hline & $\operatorname{Re}\left(C_{q q}^{(1)}\right)_{p r s t}$ & cqq1Re [prst] & {$[4 \mathrm{f}-\mathrm{H}-\mathrm{S}-\mathrm{R}]$} & $\operatorname{Im}\left(C_{q q}^{(1)}\right)_{p r s t}$ & cqq1Im [prst] & {$[4 f-H-S-I]$} \\
\hline & $\operatorname{Re}\left(C_{q q}^{(3)}\right)_{p r s t}$ & cqq3Re [prst] & {$[4 \mathrm{f}-\mathrm{H}-\mathrm{S}-\mathrm{R}]$} & $\operatorname{Im}\left(C_{q q}^{(3)}\right)_{p r s t}$ & cqq3Im [prst] & {$[4 \mathrm{f}-\mathrm{H}-\mathrm{S}-\mathrm{I}]$} \\
\hline & $\operatorname{Re}\left(C_{l q}^{(1)}\right)_{p r s t}$ & clq1Re [prst] & {$[4 \mathrm{f}-\mathrm{H}-\mathrm{R}]$} & $\operatorname{Im}\left(C_{l q}^{(1)}\right)_{p r s t}$ & clq1Im [prst] & {$[4 f-H-I]$} \\
\hline & $\operatorname{Re}\left(C_{l q}^{(3)}\right)_{p r s t}$ & clq3Re [prst] & {$[4 \mathrm{f}-\mathrm{H}-\mathrm{R}]$} & $\operatorname{Im}\left(C_{l q}^{(3)}\right)_{p r s t}$ & clq3Im [prst] & {$[4 \mathrm{f}-\mathrm{H}-\mathrm{I}]$} \\
\hline \multirow{7}{*}{$\mathcal{L}_{6}^{(8 b)}$} & $\operatorname{Re}\left(C_{e e}\right)_{p r s t}$ & ceeRe [prst] & {$[4 f-e e-R]$} & $\operatorname{Im}\left(C_{e e}\right)_{p r s t}$ & ceeIm[prst] & {$[4 f-e e-I]$} \\
\hline & $\operatorname{Re}\left(C_{u u}\right)_{p r s t}$ & cuuRe [prst] & {$[4 f-H-S-R]$} & $\operatorname{Im}\left(C_{u u}\right)_{p r s t}$ & cuuIm [prst] & {$[4 \mathrm{f}-\mathrm{H}-\mathrm{S}-\mathrm{I}]$} \\
\hline & $\operatorname{Re}\left(C_{d d}\right)_{p r s t}$ & cddRe [prst] & {$[4 \mathrm{f}-\mathrm{H}-\mathrm{S}-\mathrm{R}]$} & $\operatorname{Im}\left(C_{d d}\right)_{p r s t}$ & cddIm [prst] & {$[4 \mathrm{f}-\mathrm{H}-\mathrm{S}-\mathrm{I}]$} \\
\hline & $\operatorname{Re}\left(C_{e u}\right)_{p r s t}$ & ceuRe [prst] & {$[4 \mathrm{f}-\mathrm{H}-\mathrm{R}]$} & $\operatorname{Im}\left(C_{e u}\right)_{p r s t}$ & ceuIm [prst] & {$[4 f-H-I]$} \\
\hline & $\operatorname{Re}\left(C_{e d}\right)_{p r s t}$ & cedRe [prst] & {$[4 \mathrm{f}-\mathrm{H}-\mathrm{R}]$} & $\operatorname{Im}\left(C_{e d}\right)_{p r s t}$ & cedIm [prst] & {$[4 \mathrm{f}-\mathrm{H}-\mathrm{I}]$} \\
\hline & $\operatorname{Re}\left(C_{u d}^{(1)}\right)_{p r s t}$ & cud1Re [prst] & {$[4 \mathrm{f}-\mathrm{H}-\mathrm{R}]$} & $\operatorname{Im}\left(C_{u d}^{(1)}\right)_{p r s t}$ & cud1Im [prst] & {$[4 \mathrm{f}-\mathrm{H}-\mathrm{I}]$} \\
\hline & $\operatorname{Re}\left(C_{u d}^{(8)}\right)_{p r s t}$ & cud8Re [prst] & {$[4 \mathrm{f}-\mathrm{H}-\mathrm{R}]$} & $\operatorname{Im}\left(C_{u d}^{(8)}\right)_{p r s t}$ & cud8Im [prst] & {$[4 \mathrm{f}-\mathrm{H}-\mathrm{I}]$} \\
\hline \multirow{8}{*}{$\mathcal{L}_{6}^{(8 c)}$} & $\operatorname{Re}\left(C_{l e}\right)_{p r s t}$ & cleRe [prst] & {$[4 \mathrm{f}-\mathrm{H}-\mathrm{R}]$} & $\operatorname{Im}\left(C_{l e}\right)_{p r s t}$ & cleIm[prst] & {$[4 \mathrm{f}-\mathrm{H}-\mathrm{I}]$} \\
\hline & $\operatorname{Re}\left(C_{l u}\right)_{p r s t}$ & cluRe [prst] & {$[4 \mathrm{f}-\mathrm{H}-\mathrm{R}]$} & $\operatorname{Im}\left(C_{l u}\right)_{p r s t}$ & cluIm [prst] & {$[4 \mathrm{f}-\mathrm{H}-\mathrm{I}]$} \\
\hline & $\operatorname{Re}\left(C_{l d}\right)_{p r s t}$ & cldRe [prst] & {$[4 \mathrm{f}-\mathrm{H}-\mathrm{R}]$} & $\operatorname{Im}\left(C_{l d}\right)_{p r s t}$ & cldIm [prst] & {$[4 \mathrm{f}-\mathrm{H}-\mathrm{I}]$} \\
\hline & $\operatorname{Re}\left(C_{q e}\right)_{p r s t}$ & cqeRe [prst] & {$[4 f-H-R]$} & $\operatorname{Im}\left(C_{q e}\right)_{p r s t}$ & cqeIm [prst] & {$[4 f-H-I]$} \\
\hline & $\operatorname{Re}\left(C_{q u}^{(1)}\right)_{p r s t}$ & cqu1Re [prst] & {$[4 \mathrm{f}-\mathrm{H}-\mathrm{R}]$} & $\operatorname{Im}\left(C_{q u}^{(1)}\right)_{p r s t}$ & cqu1Im [prst] & {$[4 \mathrm{f}-\mathrm{H}-\mathrm{I}]$} \\
\hline & $\operatorname{Re}\left(C_{q u}^{(8)}\right)_{p r s t}$ & cqu8Re [prst] & {$[4 \mathrm{f}-\mathrm{H}-\mathrm{R}]$} & $\operatorname{Im}\left(C_{q u}^{(8)}\right)_{p r s t}$ & cqu8Im [prst] & {$[4 \mathrm{f}-\mathrm{H}-\mathrm{I}]$} \\
\hline & $\operatorname{Re}\left(C_{q d}^{(1)}\right)_{p r s t}$ & cqd1Re [prst] & {$[4 \mathrm{f}-\mathrm{H}-\mathrm{R}]$} & $\operatorname{Im}\left(C_{q d}^{(1)}\right)_{p r s t}$ & cqd1Im [prst] & {$[4 \mathrm{f}-\mathrm{H}-\mathrm{I}]$} \\
\hline & $\operatorname{Re}\left(C_{q d}^{(8)}\right)_{p r s t}$ & cqd8Re [prst] & {$[4 \mathrm{f}-\mathrm{H}-\mathrm{R}]$} & $\operatorname{Im}\left(C_{q d}^{(8)}\right)_{p r s t}$ & cqd8Im [prst] & {$[4 \mathrm{f}-\mathrm{H}-\mathrm{I}]$} \\
\hline \multirow{5}{*}{$\mathcal{L}_{6}^{(8 d)}$} & $\operatorname{Re}\left(C_{l e d q}\right)_{p r s t}$ & cledqRe [prst] & {$[4 f-\mathrm{NH}-\mathrm{R}]$} & $\operatorname{Im}\left(C_{l e d q}\right)_{p r s t}$ & cledqIm [prst] & {$[4 \mathrm{f}-\mathrm{NH}-\mathrm{I}]$} \\
\hline & $\operatorname{Re}\left(C_{q u q d}^{(1)}\right)_{p r s t}$ & cquqd1Re [prst] & {$[4 f-N H-R]$} & $\operatorname{Im}\left(C_{q u q d}^{(1)}\right)_{p r s t}$ & cquqd1Im [prst] & {$[4 f-N H-I]$} \\
\hline & $\operatorname{Re}\left(C_{q u q d}^{(8)}\right)_{p r s t}$ & cquqd8Re [prst] & {$[4 \mathrm{f}-\mathrm{NH}-\mathrm{R}]$} & $\operatorname{Im}\left(C_{\text {quqd }}^{(8)}\right)_{\text {prst }}$ & cquqd8Im [prst] & {$[4 \mathrm{f}-\mathrm{NH}-\mathrm{I}]$} \\
\hline & $\operatorname{Re}\left(C_{\text {lequ }}^{(1)}\right)_{p r s t}$ & clequ1Re [prst] & {$[4 \mathrm{f}-\mathrm{NH}-\mathrm{R}]$} & $\operatorname{Im}\left(C_{\text {lequ }}^{(1)}\right)_{\text {prst }}$ & clequ1Im [prst] & {$[4 \mathrm{f}-\mathrm{NH}-\mathrm{I}]$} \\
\hline & $\operatorname{Re}\left(C_{\text {lequ }}^{(3)}\right)_{\text {prst }}$ & clequ3Re [prst] & {$[4 \mathrm{f}-\mathrm{NH}-\mathrm{R}]$} & $\operatorname{Im}\left(C_{\text {lequ }}^{(3)}\right)_{\text {prst }}$ & clequ3Im [prst] & {$[4 \mathrm{f}-\mathrm{NH}-\mathrm{I}]$} \\
\hline
\end{tabular}

Table 18. The 2484 independent parameters in $\mathcal{L}_{6}^{(5,6,7,8)}$ defined in the general flavor model, see section 3.1. The indices strings $[\mathrm{pr}]$, [prst] take values in the sets indicated, that are defined in table 19 . 


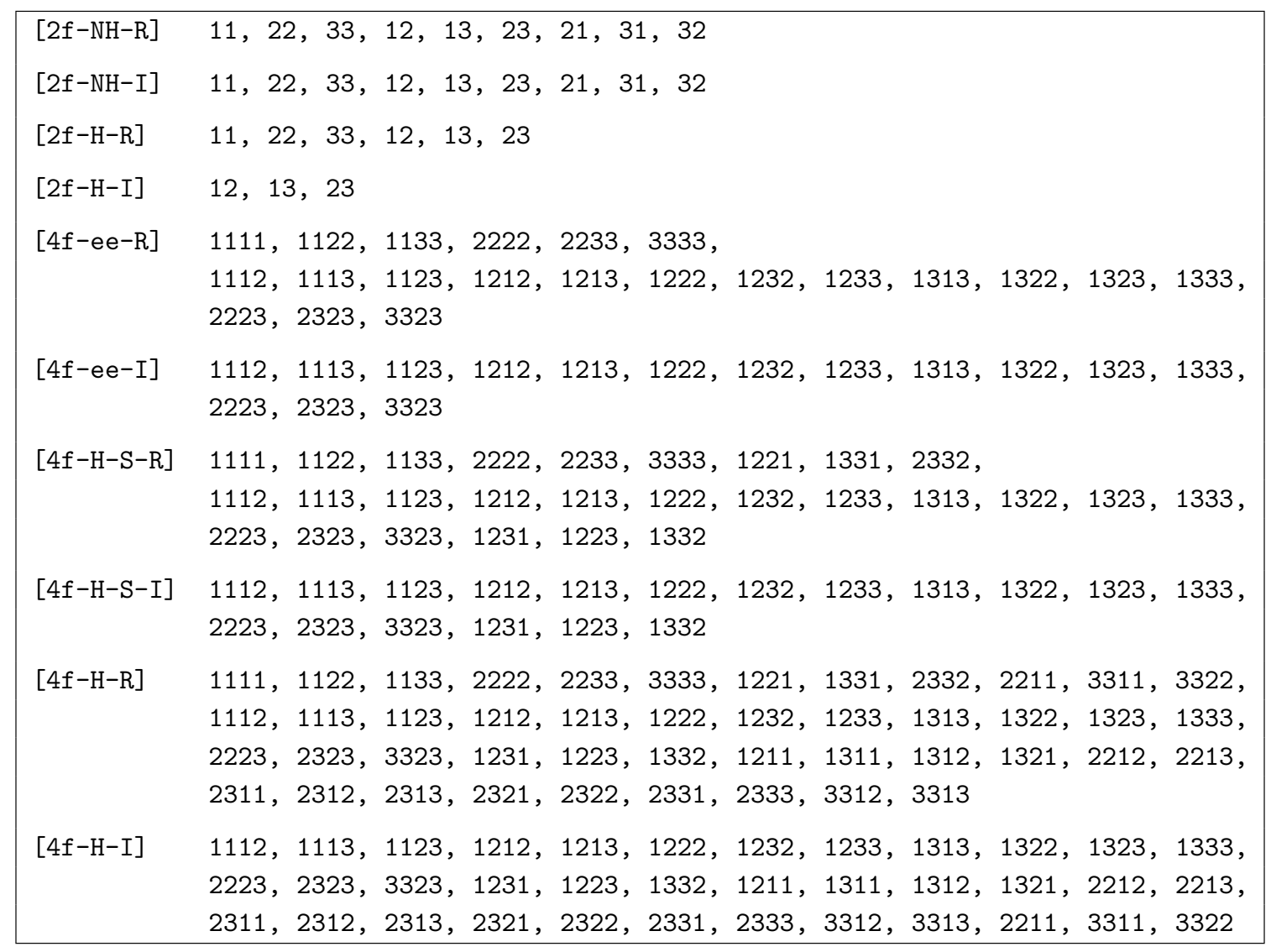

Table 19. Sets of indices implemented for each category of fermionic operator in the general model. 


\begin{tabular}{|c|c|c|c|c|c|c|c|c|}
\hline \multicolumn{9}{|c|}{ Fermionic SMEFT parameters in SMEFTsim U35 } \\
\hline \multirow{2}{*}{$\mathcal{L}_{6}^{(5)}$} & $\operatorname{Re} C_{e H}$ & ceHRe & $\operatorname{Re} C_{u H}$ & cuHRe & $\operatorname{Re} C_{d H}$ & $\mathrm{cdHRe}$ & & \\
\hline & $\operatorname{Im} C_{e H}$ & ceHIm & $\operatorname{Im} C_{u H}$ & cuHIm & $\operatorname{Im} C_{d H}$ & cdHIm & & \\
\hline \multirow{4}{*}{$\mathcal{L}_{6}^{(6)}$} & $\operatorname{Re} C_{e W}$ & ceWRe & $\operatorname{Re} C_{e B}$ & ceBRe & $\operatorname{Re} C_{u G}$ & cuGRe & $\operatorname{Re} C_{u W}$ & cuWRe \\
\hline & $\operatorname{Im} C_{e W}$ & ceWIm & $\operatorname{Im} C_{e B}$ & ceBIm & $\operatorname{Im} C_{u G}$ & cuGIm & $\operatorname{Im} C_{u W}$ & cuWIm \\
\hline & $\operatorname{Re} C_{u B}$ & cuBRe & $\operatorname{Re} C_{d G}$ & cdGRe & $\operatorname{Re} C_{d W}$ & cdWRe & $\operatorname{Re} C_{d B}$ & cdBRe \\
\hline & $\operatorname{Im} C_{u B}$ & cuBIm & $\operatorname{Im} C_{d G}$ & cdGIm & $\operatorname{Im} C_{d W}$ & cdWIm & $\operatorname{Im} C_{d B}$ & $\mathrm{cdBIm}$ \\
\hline \multirow{3}{*}{$\mathcal{L}_{6}^{(7)}$} & $C_{H l}^{(1)}$ & $\mathrm{cHl1}$ & $C_{H q}^{(1)}$ & cHq1 & $C_{H u}$ & $\mathrm{cHu}$ & $C_{H e}$ & $\mathrm{cHe}$ \\
\hline & $C_{H l}^{(3)}$ & $\mathrm{cH} 13$ & $C_{H q}^{(3)}$ & $\mathrm{cHq}_{3}$ & $C_{H d}$ & cHd & & \\
\hline & $\operatorname{Re} C_{H u d}$ & cHudRe & $\operatorname{Im} C_{H u d}$ & cHudIm & & & & \\
\hline \multirow{2}{*}{$\mathcal{L}_{6}^{(8 a)}$} & $C_{l l}$ & $\mathrm{cll}$ & $C_{q q}^{(1)}$ & cqq1 & $C_{q q}^{(3)}$ & cqq3 & $C_{l q}^{(1)}$ & clq1 \\
\hline & $C_{l l}^{\prime}$ & cll1 & $C_{q q}^{(1) \prime}$ & cqq11 & $C_{q q}^{(3) \prime}$ & cqq31 & $C_{l q}^{(3)}$ & clq3 \\
\hline \multirow{3}{*}{$\mathcal{L}_{6}^{(8 b)}$} & $C_{u u}$ & cuu & $C_{d d}$ & cdd & $C_{e u}$ & ceu & $C_{u d}^{(1)}$ & cud1 \\
\hline & $C_{u u}^{\prime}$ & cuu1 & $C_{d d}^{\prime}$ & cdd 1 & $C_{e d}$ & ced & $C_{u d}^{(8)}$ & cud8 \\
\hline & $C_{e e}$ & cee & & & & & & \\
\hline \multirow{2}{*}{$\mathcal{L}_{6}^{(8 c)}$} & $C_{l e}$ & $\mathrm{cle}$ & $C_{l u}$ & $\mathrm{clu}$ & $C_{q u}^{(1)}$ & cqu1 & $C_{q d}^{(1)}$ & cqd1 \\
\hline & $C_{q e}$ & cqe & $C_{l d}$ & $\mathrm{cld}$ & $C_{q u}^{(8)}$ & cqu8 & $C_{q d}^{(8)}$ & cqd8 \\
\hline \multirow{4}{*}{$\mathcal{L}_{6}^{(8 d)}$} & $\operatorname{Re} C_{q u q d}^{(1)}$ & cquqd1Re & $\operatorname{Re} C_{q u q d}^{(8)}$ & cquqd8Re & $\operatorname{Im} C_{q u q d}^{(1)}$ & cquqd1Im & $\operatorname{Im} C_{q u q d}^{(8)}$ & cquqd8Im \\
\hline & $\operatorname{Re} C_{q u q d}^{(1)^{\prime}}$ & cquqd11Re & $\operatorname{Re} C_{q u q d}^{(8)^{\prime}}$ & cquqd81Re & $\operatorname{Im} C_{q u q d}^{(1)^{\prime}}$ & cquqd11Im & $\operatorname{Im} C_{q u q d}^{(8)^{\prime}}$ & cquqd81 Im \\
\hline & $\operatorname{Re} C_{l e q u}^{(1)}$ & clequ1Re & $\operatorname{Re} C_{l e q u}^{(3)}$ & clequ3Re & $\operatorname{Im} C_{l e q u}^{(1)}$ & clequ1Im & $\operatorname{Im} C_{l e q u}^{(3)}$ & clequ3Im \\
\hline & $\operatorname{Re} C_{l e d q}$ & cledqRe & $\operatorname{Im} C_{l e d q}$ & cledqIm & & & & \\
\hline
\end{tabular}

Table 20. The 70 independent parameters in $\mathcal{L}_{6}^{(5,6,7,8)}$ defined in the U35 model, see section 3.2 . 


\begin{tabular}{|c|c|c|c|c|c|c|c|c|}
\hline \multicolumn{9}{|c|}{ Fermionic SMEFT parameters in SMEFTsim MFV } \\
\hline $\mathcal{L}_{6}^{(5)}$ & $\begin{array}{c}C_{e H} \\
\left(\Delta^{u} C_{u H}\right)\end{array}$ & $\begin{array}{c}\text { ceH } \\
\text { DeltaucuH }\end{array}$ & $\begin{array}{c}C_{u H}^{(0)} \\
\left(\Delta^{d} C_{u H}\right)\end{array}$ & $\begin{array}{c}\text { cuHo } \\
\text { DeltadcuH }\end{array}$ & $\begin{array}{c}C_{d H}^{(0)} \\
\left(\Delta^{u} C_{d H}\right)\end{array}$ & $\begin{array}{c}\text { cdHO } \\
\text { DeltaucdH }\end{array}$ & $\left(\Delta^{d} C_{d H}\right)$ & DeltadcdH \\
\hline \multirow{5}{*}{$\mathcal{L}_{6}^{(6)}$} & $C_{e W}$ & $\mathrm{ceW}$ & $C_{e B}$ & $\mathrm{ceB}$ & $C_{u G}^{(0)}$ & cuGO & $C_{u W}^{(0)}$ & cuWO \\
\hline & $C_{u B}^{(0)}$ & cuBO & $C_{d G}^{(0)}$ & cdGO & $C_{d W}^{(0)}$ & cdW0 & $C_{d B}^{(0)}$ & $\mathrm{cdBO}$ \\
\hline & $\left(\Delta^{u} C_{u G}\right)$ & DeltaucuG & $\left(\Delta^{d} C_{u G}\right)$ & DeltadcuG & $\left(\Delta^{u} C_{d G}\right)$ & DeltaucdG & $\left(\Delta^{d} C_{d G}\right)$ & DeltadcdG \\
\hline & $\left(\Delta^{u} C_{u W}\right)$ & DeltaucuW & $\left(\Delta^{d} C_{u W}\right)$ & DeltadcuW & $\left(\Delta^{u} C_{d W}\right)$ & DeltaucdW & $\left(\Delta^{d} C_{d W}\right)$ & DeltadcdW \\
\hline & $\left(\Delta^{u} C_{u B}\right)$ & DeltaucuB & $\left(\Delta^{d} C_{u B}\right)$ & DeltadcuB & $\left(\Delta^{u} C_{d B}\right)$ & DeltaucdB & $\left(\Delta^{d} C_{d B}\right)$ & DeltadcdB \\
\hline \multirow{4}{*}{$\mathcal{L}_{6}^{(7)}$} & $C_{H l}^{(1)}$ & $\mathrm{cHl1}$ & $C_{H q}^{(1)(0)}$ & cHq10 & $C_{H u}^{(0)}$ & $\mathrm{cHuO}$ & $C_{H e}$ & $\mathrm{cHe}$ \\
\hline & $C_{H l}^{(3)}$ & $\mathrm{cH} 13$ & $C_{H q}^{(3)(0)}$ & $\mathrm{cHq} 30$ & $C_{H d}^{(0)}$ & $\mathrm{cHdO}$ & $C_{H u d}^{(0)}$ & cHudO \\
\hline & $\left(\Delta^{u} C_{H q}^{(1)}\right)$ & DeltaucHq1 & $\left(\Delta^{d} C_{H q}^{(1)}\right)$ & DeltadcHq1 & $\left(\Delta^{u} C_{H q}^{(3)}\right)$ & DeltaucHq3 & $\left(\Delta^{d} C_{H q}^{(3)}\right)$ & DeltadcHq3 \\
\hline & $\left(\Delta C_{H u}\right)$ & DeltacHu & $\left(\Delta C_{H d}\right)$ & DeltacHd & & & & \\
\hline \multirow{5}{*}{$\mathcal{L}_{6}^{(8 a)}$} & $C_{l l}$ & $\mathrm{cll}$ & $C_{q q}^{(1)(0)}$ & cqq10 & $C_{q q}^{(3)(0)}$ & cqq30 & $C_{l q}^{(1)(0)}$ & clq10 \\
\hline & $C_{l l}^{\prime}$ & cll1 & $C_{q q}^{(1) \prime(0)}$ & cqq110 & $C_{q q}^{(3) \prime(0)}$ & cqq310 & $C_{l q}^{(3)(0)}$ & clq30 \\
\hline & $\left(\Delta^{u} C_{q q}^{(1)}\right)$ & Deltaucqq1 & $\left(\Delta^{d} C_{q q}^{(1)}\right)$ & Deltadcqq1 & $\left(\Delta^{u} C_{q q}^{(3)}\right)$ & Deltaucqq3 & $\left(\Delta^{d} C_{q q}^{(3)}\right)$ & Deltadcqq3 \\
\hline & $\left(\Delta^{u} C_{q q}^{(1) \prime}\right)$ & Deltaucqq11 & $\left(\Delta^{d} C_{q q}^{(1) \prime}\right)$ & Deltadcqq11 & $\left(\Delta^{u} C_{q q}^{(3) \prime}\right)$ & Deltaucqq31 & $\left(\Delta^{d} C_{q q}^{(3) \prime}\right)$ & Deltadcqq31 \\
\hline & $\left(\Delta^{u} C_{l q}^{(1)}\right)$ & Deltauclq1 & $\left(\Delta^{d} C_{l q}^{(1)}\right)$ & Deltadclq1 & $\left(\Delta^{u} C_{l q}^{(3)}\right)$ & Deltauclq3 & $\left(\Delta^{d} C_{l q}^{(3)}\right)$ & Deltadclq3 \\
\hline \multirow{5}{*}{$\mathcal{L}_{6}^{(8 b)}$} & $C_{u u}^{(0)}$ & cuuO & $C_{d d}^{(0)}$ & $\operatorname{cdd} 0$ & $C_{e u}^{(0)}$ & ceu0 & $C_{u d}^{(1)(0)}$ & cud10 \\
\hline & $C_{u u}^{\prime(0)}$ & cuu10 & $C_{d d}^{\prime(0)}$ & cdd10 & $C_{e d}^{(0)}$ & ced0 & $C_{u d}^{(8)(0)}$ & cud80 \\
\hline & $C_{e e}$ & cee & $\left(\Delta C_{e u}\right)$ & Deltaceu & $\left(\Delta C_{e d}\right)$ & Deltaced & & \\
\hline & $\left(\Delta^{u} C_{u u}\right)$ & Deltaucuu & $\left(\Delta^{d} C_{d d}\right)$ & Deltadcdd & $\left(\Delta^{u} C_{u u}^{\prime}\right)$ & Deltaucuu1 & $\left(\Delta^{d} C_{d d}^{\prime}\right)$ & Deltadcdd1 \\
\hline & $\left(\Delta^{u} C_{u d}^{(1)}\right)$ & Deltaucud1 & $\left(\Delta^{d} C_{u d}^{(1)}\right)$ & Deltadcud1 & $\left(\Delta^{u} C_{u d}^{(8)}\right)$ & Deltaucud8 & $\left(\Delta^{d} C_{u d}^{(8)}\right)$ & Deltadcud8 \\
\hline \multirow{7}{*}{$\mathcal{L}_{6}^{(8 c)}$} & $C_{l e}$ & $\mathrm{cle}$ & $C_{l u}^{(0)}$ & cluo & $C_{l d}^{(0)}$ & $\operatorname{cld} 0$ & $C_{q e}^{(0)}$ & cqeo \\
\hline & $C_{q u}^{(1)(0)}$ & cqu10 & $C_{q d}^{(1)(0)}$ & cqd10 & $C_{q u}^{(8)(0)}$ & cqu 80 & $C_{q d}^{(8)(0)}$ & cqd80 \\
\hline & $C_{q u}^{(1) \prime(0)}$ & cqu110 & $C_{q d}^{(1) \prime(0)}$ & cqd110 & $C_{q u}^{(8) \prime(0)}$ & cqu810 & $C_{q d}^{(8) \prime(0)}$ & cqd810 \\
\hline & $\left(\Delta_{1}^{u} C_{q u}^{(1)}\right)$ & Delta1ucqu1 & $\left(\Delta_{1}^{d} C_{q u}^{(1)}\right)$ & Delta1dcqu1 & $\left(\Delta_{2} C_{q u}^{(1)}\right)$ & Delta2cqu1 & $\left(\Delta C_{l u}\right)$ & Deltaclu \\
\hline & $\left(\Delta_{1}^{u} C_{q u}^{(8)}\right)$ & Delta1ucqu8 & $\left(\Delta_{1}^{d} C_{q u}^{(8)}\right)$ & Delta1dcqu8 & $\left(\Delta_{2} C_{q u}^{(8)}\right)$ & Delta2cqu8 & $\left(\Delta C_{l d}\right)$ & Deltacld \\
\hline & $\left(\Delta_{1}^{u} C_{q d}^{(1)}\right)$ & Delta1ucqd1 & $\left(\Delta_{1}^{d} C_{q d}^{(1)}\right)$ & Delta1dcqd1 & $\left(\Delta_{2} C_{q d}^{(1)}\right)$ & Delta2cqd1 & $\left(\Delta^{u} C_{q e}\right)$ & Deltaucqe \\
\hline & $\left(\Delta_{1}^{u} C_{q d}^{(8)}\right)$ & Delta1ucqd8 & $\left(\Delta_{1}^{d} C_{q d}^{(8)}\right)$ & Delta1dcqd8 & $\left(\Delta_{2} C_{q d}^{(8)}\right)$ & Delta2cqd8 & $\left(\Delta^{d} C_{q e}\right)$ & Deltadcqe \\
\hline \multirow{4}{*}{$\mathcal{L}_{6}^{(8 d)}$} & $C_{l e q u}^{(1)(0)}$ & clequ10 & $C_{l e q u}^{(3)(0)}$ & clequ30 & $C_{l e d q}^{(0)}$ & cledq0 & & \\
\hline & $C_{q u q d}^{(1)}$ & cquqd1 & $C_{q u q d}^{(8)}$ & cquqd8 & $C_{q u q d}^{(1)^{\prime}}$ & cquqd11 & $C_{q u q d}^{(8)^{\prime}}$ & cquqd81 \\
\hline & $\left(\Delta^{u} C_{l e d q}\right)$ & Deltaucledq & $\left(\Delta^{d} C_{l e d q}\right)$ & Deltadcledq & & & & \\
\hline & $\left(\Delta^{u} C_{l e q u}^{(1)}\right)$ & Deltauclequ1 & $\left(\Delta^{d} C_{\text {lequ }}^{(1)}\right)$ & Deltadclequ1 & $\left(\Delta^{u} C_{l e q u}^{(3)}\right)$ & Deltauclequ3 & $\left(\Delta^{d} C_{l e q u}^{(3)}\right)$ & Deltadclequ3 \\
\hline
\end{tabular}

Table 21. The 121 independent parameters in $\mathcal{L}_{6}^{(5,6,7,8)}$ defined in the MFV model, see section 3.3. 


\begin{tabular}{|c|c|c|c|c|c|c|c|c|}
\hline \multicolumn{9}{|c|}{ Fermionic SMEFT parameters in SMEFTsim top } \\
\hline $\mathcal{L}_{6}^{(5)}$ & $\begin{array}{c}\operatorname{Re}\left(C_{e H}\right)_{p p} \\
\operatorname{Re} C_{u H} \\
\operatorname{Re} C_{d H}\end{array}$ & $\begin{array}{c}\text { ceHRe [pp] } \\
\text { cuHRe } \\
\text { cdHRe }\end{array}$ & $\begin{array}{l}\operatorname{Im}\left(C_{e H}\right)_{p p} \\
\operatorname{Im} C_{u H} \\
\operatorname{Im} C_{d H}\end{array}$ & $\begin{array}{c}\text { ceHIm [pp] } \\
\text { cuHIm } \\
\text { cdHIm }\end{array}$ & $\begin{array}{l}\operatorname{Re} C_{t H} \\
\operatorname{Re} C_{b H}\end{array}$ & $\begin{array}{l}\text { ctHRe } \\
\text { cbHRe }\end{array}$ & $\begin{array}{l}\operatorname{Im} C_{t H} \\
\operatorname{Im} C_{b H}\end{array}$ & $\begin{array}{l}\text { ctHIm } \\
\text { cbHIm }\end{array}$ \\
\hline $\mathcal{L}_{6}^{(6)}$ & $\begin{array}{c}\operatorname{Re}\left(C_{e W}\right)_{p p} \\
\operatorname{Re} C_{u G} \\
\operatorname{Re} C_{d G} \\
\operatorname{Re} C_{u W} \\
\operatorname{Re} C_{d W} \\
\operatorname{Re} C_{u B} \\
\operatorname{Re} C_{d B}\end{array}$ & $\begin{array}{l}\text { ceWRe [pp] } \\
\text { cuGRe } \\
\text { cdGRe } \\
\text { cuWRe } \\
\text { cdWRe } \\
\text { cuBRe } \\
\text { cdBRe }\end{array}$ & $\begin{array}{c}\operatorname{Im}\left(C_{e W}\right)_{p p} \\
\operatorname{Im} C_{u G} \\
\operatorname{Im} C_{d G} \\
\operatorname{Im} C_{u W} \\
\operatorname{Im} C_{d W} \\
\operatorname{Im} C_{u B} \\
\operatorname{Im} C_{d B}\end{array}$ & $\begin{array}{l}\text { ceWIm }[\mathrm{pp}] \\
\text { cuGIm } \\
\text { cdGIm } \\
\text { cuWIm } \\
\text { cdWIm } \\
\text { cuBIm } \\
\text { cdBIm }\end{array}$ & $\begin{array}{c}\operatorname{Re}\left(C_{e B}\right)_{p p} \\
\operatorname{Re} C_{t G} \\
\operatorname{Re} C_{b G} \\
\operatorname{Re} C_{t W} \\
\operatorname{Re} C_{b W} \\
\operatorname{Re} C_{t B} \\
\operatorname{Re} C_{b B}\end{array}$ & $\begin{array}{l}\text { ceBRe }[\mathrm{pp}] \\
\text { ctGRe } \\
\text { cbGRe } \\
\text { ctWRe } \\
\text { cbWRe } \\
\text { ctBRe } \\
\text { cbBRe }\end{array}$ & $\begin{array}{c}\operatorname{Im}\left(C_{e B}\right)_{p p} \\
\operatorname{Im} C_{t G} \\
\operatorname{Im} C_{b G} \\
\operatorname{Im} C_{t W} \\
\operatorname{Im} C_{b W} \\
\operatorname{Im} C_{t B} \\
\operatorname{Im} C_{b B}\end{array}$ & $\begin{array}{l}\text { ceBIm [pp] } \\
\text { ctGIm } \\
\text { cbGIm } \\
\text { ctWIm } \\
\text { cbWIm } \\
\text { ctBIm } \\
\text { cbBIm }\end{array}$ \\
\hline $\mathcal{L}_{6}^{(7)}$ & $\begin{array}{c}\left(C_{H l}^{(1)}\right)_{p p} \\
C_{H q}^{(1)} \\
C_{H q}^{(3)} \\
\operatorname{Re} C_{H u d}\end{array}$ & $\begin{array}{c}\text { cH11 [pp] } \\
\text { cHj1 } \\
\text { cHj3 } \\
\text { cHudRe }\end{array}$ & $\begin{array}{c}\left(C_{H l}^{(3)}\right)_{p p} \\
C_{H Q}^{(1)} \\
C_{H Q}^{(3)} \\
\operatorname{Im} C_{H u d}\end{array}$ & $\begin{array}{c}\text { cH13 [pp] } \\
\text { cHQ1 } \\
\text { cHQ3 } \\
\text { cHudIm }\end{array}$ & $\begin{array}{c}\left(C_{H e}\right)_{p p} \\
C_{H u} \\
C_{H d} \\
\operatorname{Re} C_{H t b}\end{array}$ & $\begin{array}{c}\mathrm{cHe}[\mathrm{pp}] \\
\mathrm{cHu} \\
\mathrm{cHd} \\
\text { cHtbRe }\end{array}$ & $\begin{array}{c}C_{H t} \\
C_{H b} \\
\operatorname{Im} C_{H t b}\end{array}$ & $\begin{array}{c}\mathrm{cHt} \\
\mathrm{cHbq} \\
\mathrm{cHtbIm}\end{array}$ \\
\hline $\mathcal{L}_{6}^{(8 a)}$ & $\begin{array}{l}\left(C_{l l}\right)_{p r s t} \\
\left(C_{l q}^{(1)}\right)_{p p} \\
C_{q q}^{(1,1)} \\
C_{q q}^{(3,1)} \\
C_{Q Q}^{(1)} \\
\end{array}$ & $\begin{array}{c}\text { cll [prst] } \\
\text { clj1 [pp] } \\
\text { cjj11 } \\
\text { cjj31 } \\
\text { cQQ1 }\end{array}$ & $\begin{array}{c}\text { prst }=\{111 \\
\left(C_{l Q}^{(1)}\right)_{p p} \\
C_{q q}^{(1,8)} \\
C_{q q}^{(3,8)} \\
C_{Q Q}^{(8)} \\
\end{array}$ & $\begin{array}{c}\text { 2222, 3333, } \\
\text { cQ11 [pp] } \\
\text { cjj18 } \\
\text { cjj38 } \\
\text { cQQ8 }\end{array}$ & $\begin{array}{c}1122,1133, \\
\left(C_{l q}^{(3)}\right)_{p p} \\
C_{Q q}^{(1,1)} \\
C_{Q q}^{(3,1)}\end{array}$ & $\begin{array}{c}233,1221,13 \\
\text { clj3 [pp] } \\
\text { cQj11 }\end{array}$ & $\begin{array}{c}1,2332\} \\
\left(C_{l Q}^{(3)}\right)_{p p} \\
C_{Q q}^{(1,8)} \\
C_{Q q}^{(3,8)}\end{array}$ & $\begin{array}{c}\text { cQ13 [pp] } \\
\text { cQj18 }\end{array}$ \\
\hline $\mathcal{L}_{6}^{(8 b)}$ & $\begin{array}{c}\left(C_{e e}\right)_{p r s t} \\
\left(C_{e u}\right)_{p p} \\
C_{u u}^{(1)} \\
C_{t u}^{(1)} \\
C_{t t} \\
C_{u d}^{(1)} \\
C_{u d}^{(8)} \\
\operatorname{Re}\left(C_{u t b d}^{(1)}\right)\end{array}$ & $\begin{array}{c}\text { cee [prst] } \\
\text { ceu [pp] } \\
\text { cuu1 } \\
\text { ctu1 } \\
\text { ctt } \\
\text { cud1 } \\
\text { cud8 } \\
\text { cutbd1Re }\end{array}$ & $\begin{array}{c}\text { prst }=\{111 \\
\left(C_{e t}\right)_{p p} \\
C_{u u}^{(8)} \\
C_{t u}^{(8)} \\
C_{t d}^{(1)} \\
C_{t d}^{(8)} \\
\operatorname{Im}\left(C_{u t b d}^{(1)}\right)\end{array}$ & $\begin{array}{c}\text { 2222, 3333, } \\
\text { cte }[\mathrm{pp}] \\
\text { cuu8 } 8 \\
\text { ctu8 } \\
\text { ctd1 } \\
\text { ctd8 } \\
\text { cutbd1 Im }\end{array}$ & $\begin{array}{c}1122,1133, \\
\left(C_{e d}\right)_{p p} \\
C_{d d}^{(1)} \\
C_{b d}^{(1)} \\
C_{b b} \\
C_{u b}^{(1)} \\
C_{u b}^{(8)} \\
\operatorname{Re}\left(C_{u t b d}^{(8)}\right)\end{array}$ & $\begin{array}{c}\text { ced [pp] } \\
\text { cdd1 } \\
\text { cbd1 } \\
\text { cbb } \\
\text { cbu1 } \\
\text { cbu8 } \\
\text { cutbd8Re }\end{array}$ & $\begin{array}{c}\left(C_{e b}\right)_{p p} \\
C_{d d}^{(8)} \\
C_{b d}^{(8)} \\
C_{t b}^{(1)} \\
C_{t b}^{(8)} \\
\operatorname{Im}\left(C_{u t b d}^{(8)}\right)\end{array}$ & $\begin{array}{c}\text { cbd8 } \\
\text { ctb1 } \\
\text { ctb8 } \\
\text { cutbd8Im }\end{array}$ \\
\hline $\mathcal{L}_{6}^{(8 c)}$ & $\begin{array}{c}\left(C_{l e}\right)_{p r s t} \\
\left(C_{l u}\right)_{p p} \\
\left(C_{q e}\right)_{p p} \\
C_{q u}^{(1)} \\
C_{q u}^{(8)} \\
\operatorname{Re}\left(C_{q Q t u}^{(1)}\right) \\
C_{q d}^{(1)} \\
C_{q d}^{(8)} \\
\operatorname{Re}\left(C_{q Q b d}^{(1)}\right)\end{array}$ & $\begin{array}{c}\text { cle [prst] } \\
\text { clu[pp] } \\
\text { cje[pp] } \\
\text { cju1 } \\
\text { cju8 } \\
\text { cjQtu1Re } \\
\text { cjd1 }\end{array}$ & $\begin{array}{c}\text { prst }=\{111 \\
\left(C_{l t}\right)_{p p} \\
\left(C_{Q e}\right)_{p p} \\
C_{Q u}^{(1)} \\
C_{Q u}^{(8)} \\
\operatorname{Im}\left(C_{q Q t u}^{(1)}\right) \\
C_{Q d}^{(1)} \\
C_{Q d}^{(8)} \\
\operatorname{Im}\left(C_{q Q b d}^{(1)}\right)\end{array}$ & $\begin{array}{c}\text { 2222, 3333,11 } \\
\text { ctl [pp] } \\
\text { cQe [pp] } \\
\text { cQu1 } \\
\text { cQu8 } \\
\text { cjQtu1Im } \\
\text { cQd1 }\end{array}$ & $\begin{array}{c}C_{q t}^{(1)} \\
C_{q t}^{(8)} \\
\operatorname{Re}\left(C_{q Q t u}^{(8)}\right) \\
C_{q b}^{(1)} \\
C_{q b}^{(8)} \\
\operatorname{Re}\left(C_{q Q b d}^{(8)}\right)\end{array}$ & $\begin{array}{c}\text { ctj1 } \\
\text { ctj8 } \\
\text { cjQtu8Re } \\
\text { cbj1 }\end{array}$ & $\begin{array}{c}C_{Q t}^{(1)} \\
C_{Q t}^{(8)} \\
\operatorname{Im}\left(C_{q Q t u}^{(8)}\right) \\
C_{Q b}^{(1)} \\
C_{Q b}^{(8)} \\
\operatorname{Im}\left(C_{q Q b d}^{(8)}\right)\end{array}$ & $\begin{array}{c}\text { cQt1 } \\
\text { cQt8 } \\
\text { cjQtu8Im } \\
\text { cQb1 }\end{array}$ \\
\hline $\mathcal{L}_{6}^{(8 d)}$ & $\begin{array}{c}\operatorname{Re}\left(C_{\text {ledq }}\right)_{p p} \\
\operatorname{Re}\left(C_{\text {lequ }}^{(1)}\right)_{p p} \\
\operatorname{Re}\left(C_{\text {lequ }}^{(3)}\right)_{p p} \\
\operatorname{Re} C_{q u q d}^{(1)} \\
\operatorname{Re} C_{q u q d}^{(8)} \\
\operatorname{Re} C_{q u q d}^{(1) \prime} \\
\operatorname{Re} C_{Q t q d}^{(1)} \\
\operatorname{Re} C_{Q t q d}^{(8)} \\
\operatorname{Re} C_{q t Q d}^{(1)} \\
\operatorname{Re} C_{q t Q d}^{(8)}\end{array}$ & $\begin{array}{c}\text { cleju3Re [pp] } \\
\text { cjujd1Re } \\
\text { cjujd8Re } \\
\text { cjujd11Re }\end{array}$ & $\begin{array}{c}\operatorname{Im}\left(C_{\text {ledq }}\right)_{p p} \\
\operatorname{Im}\left(C_{\text {lequ }}^{(1)}\right)_{p p} \\
\operatorname{Im}\left(C_{\text {lequ }}^{(3)}\right)_{p p} \\
\operatorname{Im} C_{q u q d}^{(1)} \\
\operatorname{Im} C_{q u q d}^{(8)} \\
\operatorname{Im} C_{q u q d}^{(1) \prime} \\
\operatorname{Im} C_{Q t q d}^{(1)} \\
\operatorname{Im} C_{Q t q d}^{(8)} \\
\operatorname{Im} C_{q t Q d}^{(1)} \\
\operatorname{Im} C_{q t Q d}^{(8)}\end{array}$ & $\begin{array}{c}\text { cleju3Im [pp] } \\
\text { cjujd1Im } \\
\text { cjujd8Im }\end{array}$ & $\begin{array}{c}\operatorname{Re}\left(C_{\text {lebQ } Q}\right)_{p p} \\
\operatorname{Re}\left(C_{\text {leQt }}^{(1)}\right)_{p p} \\
\operatorname{Re}\left(C_{\text {leQt }}^{(3)}\right)_{p p} \\
\operatorname{Re} C_{Q t Q b}^{(1)} \\
\operatorname{Re} C_{Q t Q b}^{(8)} \\
\operatorname{Re} C_{q u q d}^{(8) \prime} \\
\operatorname{Re} C_{q u Q b}^{(1)} \\
\operatorname{Re} C_{q u Q b}^{(8)} \\
\operatorname{Re} C_{Q u q b}^{(1)} \\
\operatorname{Re} C_{Q u q b}^{(8)}\end{array}$ & $\begin{array}{l}\text { cQtQb1Re } \\
\text { cQtQb8Re } \\
\text { cjujd81Re }\end{array}$ & $\begin{array}{c}\operatorname{Im}\left(C_{l e b Q}\right)_{p p} \\
\operatorname{Im}\left(C_{l e Q t}^{(1)}\right)_{p p} \\
\operatorname{Im}\left(C_{l e Q t}^{(3)}\right)_{p p} \\
\operatorname{Im} C_{Q t Q b}^{(1)} \\
\operatorname{Im} C_{Q t Q b}^{(8)} \\
\operatorname{Im} C_{q u q d}^{(8) \prime} \\
\operatorname{Im} C_{q u Q b}^{(1)} \\
\operatorname{Im} C_{q u Q b}^{(8)} \\
\operatorname{Im} C_{Q u q b}^{(1)} \\
\operatorname{Im} C_{Q u q b}^{(8)}\end{array}$ & $\begin{array}{c}\text { clebQIm [pp] } \\
\text { cleQt1Im[pp] } \\
\text { cleQt3Im[pp] } \\
\text { cQtQb1Im } \\
\text { cQtQb8Im }\end{array}$ \\
\hline
\end{tabular}

Table 22. The 260 independent parameters in $\mathcal{L}_{6}^{(5,6,7,8)}$ defined in the top model, see section 3.4. Lepton flavor indices [pp] always run over $\{11,22,33\}$. Indices [prst] take the values indicated in line. 


\begin{tabular}{|c|c|c|c|c|c|c|c|c|}
\hline \multicolumn{9}{|c|}{ Fermionic SMEFT parameters in SMEFTsim topU31 } \\
\hline \multirow{3}{*}{$\mathcal{L}_{6}^{(5)}$} & $\operatorname{Re} C_{e H}$ & ceHRe & $\operatorname{Im} C_{e H}$ & ceHIm & & & & \\
\hline & $\operatorname{Re} C_{u H}$ & cuHRe & $\operatorname{Im} C_{u H}$ & cuHIm & $\operatorname{Re} C_{t H}$ & ctHRe & $\operatorname{Im} C_{t H}$ & ctHIm \\
\hline & $\operatorname{Re} C_{d H}$ & $\mathrm{cdHRe}$ & $\operatorname{Im} C_{d H}$ & $\mathrm{cdHIm}$ & $\operatorname{Re} C_{b H}$ & $\mathrm{cbHRe}$ & $\operatorname{Im} C_{b H}$ & $\mathrm{cbHIm}$ \\
\hline \multirow{7}{*}{$\mathcal{L}_{6}^{(6)}$} & $\operatorname{Re} C_{e W}$ & ceWRe & $\operatorname{Im} C_{e W}$ & ceWIm & $\operatorname{Re} C_{e B}$ & ceBRe & $\operatorname{Im} C_{e B}$ & ceBIm \\
\hline & $\operatorname{Re} C_{u G}$ & cuGRe & $\operatorname{Im} C_{u G}$ & cuGIm & $\operatorname{Re} C_{t G}$ & ctGRe & $\operatorname{Im} C_{t G}$ & ctGIm \\
\hline & $\operatorname{Re} C_{d G}$ & cdGRe & $\operatorname{Im} C_{d G}$ & cdGIm & $\operatorname{Re} C_{b G}$ & cbGRe & $\operatorname{Im} C_{b G}$ & cbGIm \\
\hline & $\operatorname{Re} C_{u W}$ & cuWRe & $\operatorname{Im} C_{u W}$ & cuWIm & $\operatorname{Re} C_{t W}$ & ctWRe & $\operatorname{Im} C_{t W}$ & ctWIm \\
\hline & $\operatorname{Re} C_{d W}$ & cdWRe & $\operatorname{Im} C_{d W}$ & cdWIm & $\operatorname{Re} C_{b W}$ & cbWRe & $\operatorname{Im} C_{b W}$ & cbWIm \\
\hline & $\operatorname{Re} C_{u B}$ & cuBRe & $\operatorname{Im} C_{u B}$ & cuBIm & $\operatorname{Re} C_{t B}$ & ctBRe & $\operatorname{Im} C_{t B}$ & ctBIm \\
\hline & $\operatorname{Re} C_{d B}$ & cdBRe & $\operatorname{Im} C_{d B}$ & $\mathrm{cdBIm}$ & $\operatorname{Re} C_{b B}$ & $\mathrm{cbBRe}$ & $\operatorname{Im} C_{b B}$ & $\mathrm{cbBIm}$ \\
\hline \multirow{4}{*}{$\mathcal{L}_{6}^{(7)}$} & $C_{H l}^{(1)}$ & $\mathrm{cHl} 1$ & $C_{H l}^{(3)}$ & $\mathrm{cHl} 3$ & $C_{H e}$ & $\mathrm{cHe}$ & & \\
\hline & $C_{H q}^{(1)}$ & $\mathrm{cHj} 1$ & $C_{H Q}^{(1)}$ & cHQ1 & $C_{H u}$ & $\mathrm{cHu}$ & $C_{H t}$ & $\mathrm{cHt}$ \\
\hline & $C_{H q}^{(3)}$ & $\mathrm{cHj3}$ & $C_{H Q}^{(3)}$ & cHQ3 & $C_{H d}$ & $\mathrm{cHd}$ & $C_{H b}$ & $\mathrm{cHbq}$ \\
\hline & $\operatorname{Re} C_{H u d}$ & cHudRe & $\operatorname{Im} C_{H u d}$ & cHudIm & $\operatorname{Re} C_{H t b}$ & cHtbRe & $\operatorname{Im} C_{H t b}$ & cHtbIm \\
\hline \multirow{4}{*}{$\mathcal{L}_{6}^{(8 a)}$} & $C_{l l}$ & $\mathrm{cll}$ & $C_{l l}^{\prime}$ & cll1 & $C_{Q Q}^{(1)}$ & cQQ1 & $C_{Q Q}^{(8)}$ & cQQ8 \\
\hline & $C_{l q}^{(1)}$ & clj1 & $C_{l Q}^{(1)}$ & cQl1 & $C_{l q}^{(3)}$ & $\mathrm{clj3}$ & $C_{l Q}^{(3)}$ & cQ13 \\
\hline & $C_{q q}^{(1,1)}$ & cjj11 & $C_{q q}^{(1,8)}$ & $c j j 18$ & $C_{Q q}^{(1,1)}$ & $c Q j 11$ & $C_{Q q}^{(1,8)}$ & $c Q j 18$ \\
\hline & $C_{q q}^{(3,1)}$ & cjj31 & $C_{q q}^{(3,8)}$ & $c j j 38$ & $C_{Q q}^{(3,1)}$ & $c Q j 31$ & $C_{Q q}^{(3,8)}$ & $c Q j 38$ \\
\hline \multirow{7}{*}{$\mathcal{L}_{6}^{(8 b)}$} & $C_{e u}$ & ceu & $C_{e t}$ & cte & $C_{e d}$ & ced & $C_{e b}$ & cbe \\
\hline & $C_{u u}^{(1)}$ & cuu1 & $C_{u u}^{(8)}$ & cuus & $C_{d d}^{(1)}$ & cdd1 & $C_{d d}^{(8)}$ & cdd8 \\
\hline & $C_{t u}^{(1)}$ & ctu1 & $C_{t u}^{(8)}$ & ctu8 & $C_{b d}^{(1)}$ & cbd1 & $C_{b d}^{(8)}$ & cbd8 \\
\hline & $C_{t t}$ & ctt & $C_{b b}$ & $\mathrm{cbb}$ & $C_{e e}$ & cee & & \\
\hline & $C_{u d}^{(1)}$ & cud1 & $C_{t d}^{(1)}$ & $\operatorname{ctd} 1$ & $C_{u b}^{(1)}$ & cbu1 & $C_{t b}^{(1)}$ & ctb1 \\
\hline & $C_{u d}^{(8)}$ & cud8 & $C_{t d}^{(8)}$ & $\operatorname{ctd} 8$ & $C_{u b}^{(8)}$ & cbu8 & $C_{t b}^{(8)}$ & ctb8 \\
\hline & $\operatorname{Re}\left(C_{q Q b d}^{(1)}\right)$ & cjQbd1Re & $\operatorname{Im}\left(C_{q Q b d}^{(1)}\right)$ & cjQbd1Im & $\operatorname{Re}\left(C_{q Q b d}^{(8)}\right)$ & cjQbd8Re & $\operatorname{Im}\left(C_{q Q b d}^{(8)}\right)$ & cjQbd8Im \\
\hline \multirow{8}{*}{$\mathcal{L}_{6}^{(8 c)}$} & $C_{l e}$ & $\mathrm{cle}$ & $C_{q e}$ & $c j e$ & $C_{Q e}$ & cQe & & \\
\hline & $C_{l u}$ & $\mathrm{clu}$ & $C_{l t}$ & ctl & $C_{l d}$ & cld & $C_{l b}$ & $\mathrm{cbl}$ \\
\hline & $C_{q u}^{(1)}$ & cju1 & $C_{Q u}^{(1)}$ & cQu1 & $C_{q t}^{(1)}$ & $\operatorname{ctj} 1$ & $C_{Q t}^{(1)}$ & $c Q t 1$ \\
\hline & $C_{q u}^{(8)}$ & cju8 & $C_{Q u}^{(8)}$ & cQu8 & $C_{q t}^{(8)}$ & ctj8 & $C_{Q t}^{(8)}$ & cQt8 \\
\hline & $\operatorname{Re}\left(C_{q Q t u}^{(1)}\right)$ & cjQtu1Re & $\operatorname{Im}\left(C_{q Q t u}^{(1)}\right)$ & cjQtu1Im & $\operatorname{Re}\left(C_{q Q t u}^{(8)}\right)$ & cjQtu\&Re & $\operatorname{Im}\left(C_{q Q t u}^{(8)}\right)$ & cjQtu8Im \\
\hline & $C_{q d}^{(1)}$ & cjd1 & $C_{Q d}^{(1)}$ & cQd1 & $C_{q b}^{(1)}$ & $\mathrm{cbj} 1$ & $C_{Q b}^{(1)}$ & $c Q b 1$ \\
\hline & $C_{q d}^{(8)}$ & cjd8 & $C_{Q d}^{(8)}$ & cQd8 & $C_{q b}^{(8)}$ & cbj8 & $C_{Q b}^{(8)}$ & cQb8 \\
\hline & $\operatorname{Re}\left(C_{q Q b d}^{(1)}\right)$ & cjQbd1Re & $\operatorname{Im}\left(C_{q Q b d}^{(1)}\right)$ & cjQbd1Im & $\operatorname{Re}\left(C_{q Q b d}^{(8)}\right)$ & cjQbd8Re & $\operatorname{Im}\left(C_{q Q b d}^{(8)}\right)$ & cjQbd8Im \\
\hline \multirow{10}{*}{$\mathcal{L}_{6}^{(8 d)}$} & $\operatorname{Re} C_{l e d q}$ & cledjRe & $\operatorname{Im} C_{l e d q}$ & cledqIm & $\operatorname{Re} C_{l e b Q}$ & clebQRe & $\operatorname{Im} C_{l e b Q}$ & clebQIm \\
\hline & $\operatorname{Re} C_{l e q u}^{(1)}$ & cleju1Re & $\operatorname{Im} C_{l e q u}^{(1)}$ & cleju1Im & $\operatorname{Re} C_{l e Q t}^{(1)}$ & $\mathrm{cleQt1Re}$ & $\operatorname{Im} C_{l e Q t}^{(1)}$ & cleQt1Im \\
\hline & $\operatorname{Re} C_{l e q u}^{(3)}$ & cleju3Re & $\operatorname{Im} C_{l e q u}^{(3)}$ & cleju3Im & $\operatorname{Re} C_{l e Q t}^{(3)}$ & cleQt3Re & $\operatorname{Im} C_{l e Q t}^{(3)}$ & cleQt3Im \\
\hline & $\operatorname{Re} C_{q u q d}^{(1)}$ & cjujd1Re & $\operatorname{Im} C_{q u q d}^{(1)}$ & cjujd1Im & $\operatorname{Re} C_{Q t Q b}^{(1)}$ & cQtQb1Re & $\operatorname{Im} C_{Q t Q b}^{(1)}$ & cQtQb1Im \\
\hline & $\operatorname{Re} C_{q u q d}^{(8)}$ & cjujd8Re & $\operatorname{Im} C_{q u q d}^{(8)}$ & cjujd8Im & $\operatorname{Re} C_{Q t Q b}^{(8)}$ & cQtQb8Re & $\operatorname{Im} C_{Q t Q b}^{(8)}$ & cQtQb8Im \\
\hline & $\operatorname{Re} C_{q u q d}^{(1) \prime}$ & cjujd11Re & $\operatorname{Im} C_{q u q d}^{(1) \prime}$ & cjujd11Im & $\operatorname{Re} C_{q u q d}^{(8) \prime}$ & cjujd81Re & $\operatorname{Im} C_{q u q d}^{(8) \prime}$ & cjujd81Im \\
\hline & $\operatorname{Re} C_{Q t q d}^{(1)}$ & cQtjd1Re & $\operatorname{Im} C_{Q t q d}^{(1)}$ & cQtjd1Im & $\operatorname{Re} C_{q u Q b}^{(1)}$ & cjuQb1Re & $\operatorname{Im} C_{q u Q b}^{(1)}$ & cjuQb1Im \\
\hline & $\operatorname{Re} C_{Q t q d}^{(8)}$ & cQtjd8Re & $\operatorname{Im} C_{Q t q d}^{(8)}$ & cQtjd8Im & $\operatorname{Re} C_{q u Q b}^{(8)}$ & cjuQb8Re & $\operatorname{Im} C_{q u Q b}^{(8)}$ & cjuQb8Im \\
\hline & $\operatorname{Re} C_{q t Q d}^{(1)}$ & cjtQd1Re & $\operatorname{Im} C_{q t Q d}^{(1)}$ & cjtQd1Im & $\operatorname{Re} C_{Q u q b}^{(1)}$ & cQujb1Re & $\operatorname{Im} C_{Q u q b}^{(1)}$ & cQujb1Im \\
\hline & $\operatorname{Re} C_{q t Q d}^{(8)}$ & cjtQd8Re & $\operatorname{Im} C_{q t Q d}^{(8)}$ & cjtQd8Im & $\operatorname{Re} C_{Q u q b}^{(8)}$ & cQujb8Re & $\operatorname{Im} C_{Q u q b}^{(8)}$ & cQujb8Im \\
\hline
\end{tabular}

Table 23. The 167 independent parameters in $\mathcal{L}_{6}^{(5,6,7,8)}$ defined in the topU31 model, see section 3.4. 


\section{E Comparison to other SMEFT UFO models}

In this section we compare SMEFTsim with other UFO models dedicated to SMEFT studies, and provide a mapping of the common parameters. For the time being, the comparison is restricted to dim6top [36, 121] and SMEFT@NLO [37, 122], that are both based on the Warsaw basis.

For each model we summarize the main features and provide conversion tables with the parameters defined in SMEFTsim. To our knowledge, SMEFTsim is currently the only publicly available UFO model that implements linearized SMEFT corrections to propagators.

\section{E.1 dim6top}

dim6top $[36,121]$ contains LO UFO models dedicated to EFT studies in the top sector. Here we refer specifically to dim6top_LO_UFO and dim6top_LO_UFO_each_coupling_order in the version published in May 2020.

Flavor structure. dim6top is based on the recommendations provided in ref. [36], and it assumes a $\mathrm{U}(2)^{3}$ flavor symmetry in the quark sector and a $\left(\mathrm{U}(1)_{l+e}\right)^{3}$ in the lepton sector. $\mathrm{U}(2)^{3}$ breaking terms are also available and they are implemented explicitly, i.e. without promoting the quark Yukawas to spurions of the flavor symmetry. Contractions inducing both flavor-conserving and violating neutral currents are included.

All fermion masses and Yukawa couplings are neglected, except those of the top and bottom quarks of the tau lepton. The CKM is taken to be the unit matrix.

Operators implemented. dim6top contains only operators that modify the interactions of the top quark, and CP violating terms are included. Most operator definitions are identical to those in SMEFTsim top, topU31. In a few cases, the invariants implemented differ by a Fierz rotation, as detailed in ref. [36].

Input parameters. Both input schemes $\left\{\alpha_{\mathrm{em}}, m_{Z}, G_{F}\right\}$ and $\left\{m_{W}, m_{Z}, G_{F}\right\}$ are supported in dim6top. Since purely bosonic and leptonic operators are omitted, this only affects the numerical values assigned to the SM parameters and not the dependence on the Wilson coefficients.

SM loop-generated Higgs couplings. Not implemented.

dim6top matches very closely the top and topU31 versions of SMEFTsim and it can also be mapped to the general one. A correspondence with other flavor versions of SMEFTsim can only be established partially, due to incompatibilities in the assumed flavor structure.

The mapping between Wilson coefficients defined in dim6top and in the top, topU31 versions of SMEFTsim is provided in tables 24, 25. The mapping to the general version of SMEFTsim is provided in tables 26,27 . In both cases, the first table contains parameters with a one-to-one correspondence, while the second contains parameters that require a basis rotation. For example, the point $c Q 1 M 1=1, c Q 131=3$ in dim6top corresponds to $c Q 1111=4$, cQ1311=3 (or clq1Re1133=4, clq3Re1133=3) in SMEFTsim. 
Overall minus signs in the mapping are due to the fact that dim6top and SMEFTsim use opposite sign conventions for the definition of covariant derivatives. Although the operator definitions are identical, the relative sign between the $\mathcal{L}_{6}$ contribution and the corresponding SM coupling is flipped in a few cases. The physics results are identical in both models once this is accounted for. The presence of explicit Yukawa couplings in the tables is due to the different treatment of flavor symmetry breaking terms.

Wilson coefficients inducing flavor-changing neutral currents can be mapped to parameters in SMEFTsim general, and the corresponding tables are available upon request.

\section{E.2 SMEFT@NLO}

SMEFT@NLO [37, 122] is equipped for NLO QCD calculations in MadGraph5_aMC@NLO. Here we compare specifically to SMEFTatNLO v1.0 published in September 2020.

Flavor structure. SMEFT@NLO assumes a flavor symmetry $\mathrm{U}(2)_{q} \times \mathrm{U}(3)_{d} \times \mathrm{U}(2)_{u}$ in the quark sector and $\mathrm{U}(1)^{3}$ in the lepton sector, which is the same as in SMEFTsim top and in dim6top, except for the treatment of down quarks.

All fermion masses and Yukawa couplings are neglected, except those of the top quark.

Operators implemented. SMEFT@NLO contains all the operators in classes (1)-(7) and those in class (8) that contain a top quark. Terms that violate the flavor symmetry have been consistently dropped. CP violating terms are omitted.

Input parameters. SMEFT@NLO implements the $\left\{m_{W}, m_{Z}, G_{F}\right\}$ input scheme.

SM loop-generated Higgs couplings. Higgs couplings in the $m_{t} \rightarrow \infty$ limit are not implemented, but Higgs-gluon interactions can be fully reproduced at 1-loop in QCD.

Given its flavor structure, SMEFT@NLO can be directly mapped to SMEFTsim in the top, topU3l and general versions. The mapping of Wilson coefficients between SMEFT@NLO and the top, topU31 versions of SMEFTsim is provided in tables 28, 29. The mapping to the general version is provided in tables 30,31 . In both cases, the first table contains the mapping of parameters with a one-to-one correspondence, while the second contains parameters that require a basis rotation.

As for dim6top, the sign convention used in SMEFT@NLO is the opposite compared to SMEFTsim, which leads to some minus signs in the conversion. 


\begin{tabular}{|c|c|c|c|c|c|c|}
\hline \multicolumn{7}{|c|}{ SMEFTsim top vs dim6top } \\
\hline class & \multicolumn{2}{|c|}{ SMEFTsim $\leftrightarrow$ dim6top } & \multicolumn{2}{|c|}{ SMEFTsim $\leftrightarrow$ dim6top } & \multicolumn{2}{|c|}{ SMEFTsim $\leftrightarrow$ dim6top } \\
\hline $\mathcal{L}_{6}^{(5)}$ & ctHRe & $\operatorname{ctp}$ & ctHIm & $\operatorname{ctpI}$ & & \\
\hline \multirow{2}{*}{$\mathcal{L}_{6}^{(6)}$} & ctGRe & $-\operatorname{ctg}$ & $\operatorname{ctgIm}$ & $-\operatorname{ctgI}$ & & \\
\hline & cbWRe & $-\mathrm{cbW}$ & cbWIm & - cbWI & & \\
\hline \multirow{2}{*}{$\mathcal{L}_{6}^{(7)}$} & $\mathrm{cHt}$ & $\mathrm{cpt}$ & $\mathrm{cHbq}$ & $\mathrm{cpb}$ & & \\
\hline & cHtbRe & cptb & cHtbIm & cptbI & & \\
\hline \multirow{2}{*}{$\mathcal{L}_{6}^{(8 a)}$} & cQQ1 & $\begin{array}{lll}\frac{1}{2} & \mathrm{cQQ1}\end{array}$ & $c Q j 11$ & cQq11 & cQj31 & cQq13 \\
\hline & cQQ8 & $\frac{1}{2} \quad \mathrm{cQQ8}$ & $\mathrm{cQj} 18$ & cQq81 & $c Q j 38$ & cQq83 \\
\hline \multirow{3}{*}{$\mathcal{L}_{6}^{(8 b)}$} & ctt & ctt1 & ctu1 & ctu1 & ctu8 & ctu8 \\
\hline & $\operatorname{ctd} 1$ & $\operatorname{ctd} 1$ & ctb8 & ctb8 & $\operatorname{ctd} 8$ & $\operatorname{ctd} 8$ \\
\hline & ctb1 & ctb1 & cte $[\mathrm{pp}]$ & $\operatorname{cte}[\mathrm{p}]$ & & \\
\hline \multirow{4}{*}{$\mathcal{L}_{6}^{(8 c)}$} & cQu1 & cQu1 & $\operatorname{ctj1}$ & ctq1 & cQt1 & cQt1 \\
\hline & cQu8 & cQu8 & $\operatorname{ctj} 8$ & $\operatorname{ctq} 8$ & cQt8 & cQt8 \\
\hline & cQd1 & cQd1 & cQb1 & cQb1 & cQb8 & cQb8 \\
\hline & cQd8 & cQd8 & $\mathrm{CQe}[\mathrm{pp}]$ & $c Q e[p]$ & $\operatorname{ctl}[\mathrm{pp}]$ & $\operatorname{ctl}[\mathrm{p}]$ \\
\hline \multirow{4}{*}{$\mathcal{L}_{6}^{(8 d)}$} & clebQRe [pp] & cbls [p] & cleQt1Re [pp] & $\operatorname{ctls}[\mathrm{p}]$ & cleQt3Re [pp] & $\operatorname{ctlT}[\mathrm{p}]$ \\
\hline & clebQIm [pp] & $\operatorname{cblSI}[\mathrm{p}]$ & cleQt1 Im [pp] & $\operatorname{ctlsI}[\mathrm{p}]$ & cleQt3Im [pp] & $\operatorname{ctlTI}[p]$ \\
\hline & cQtQb1Re & cQtQb1 & cQtQb8Re & cQtQb8 & & \\
\hline & cQtQb1Im & cQtQb1I & cQtQb8Im & cQtQb8I & & \\
\hline \multicolumn{7}{|c|}{ SMEFTsim topU3I vs dim6top } \\
\hline $\mathcal{L}_{6}^{(8 b)}$ & cte & $\operatorname{cte}[\mathrm{p}]$ & & & & \\
\hline $\mathcal{L}_{6}^{(8 c)}$ & cQe & $c Q e[p]$ & ctl & $\operatorname{ctl}[\mathrm{p}]$ & & \\
\hline \multirow{2}{*}{$\mathcal{L}_{6}^{(8 d)}$} & clebQRe & $\mathrm{yl}[\mathrm{p}] \quad \mathrm{cblS}[\mathrm{p}]$ & cleQt1Re & yl $[p] \operatorname{ctls}[p]$ & cleQt3Re & yl [p] ctlT[p] \\
\hline & clebQIm] & yl [p] cblSI [p] & cleQt1Im & $\mathrm{yl}[\mathrm{p}] \operatorname{ctlsI}[\mathrm{p}]$ & cleQt3Im & $y 1[p] \operatorname{ctlTI}[p]$ \\
\hline
\end{tabular}

Table 24. Upper panel: conversion table between the SMEFT parameters defined in the top version of SMEFTsim and in dim6top: parameters with a one-to-one translation. Lower panel: conversion table between the topU3I version of SMEFTsim and dim6top. Only leptonic coefficients are reported, as the other parameters behave identically to the top case. Lepton flavor indices $\mathrm{p}$ take values $p=\{1,2,3\}$, light quark indices $r$ take values $r=\{1,2\}$. 


\begin{tabular}{|c|c|c|c|c|}
\hline \multicolumn{5}{|c|}{ SMEFTsim top vs dim6top } \\
\hline class & \multicolumn{2}{|c|}{ SMEFTsim $\rightarrow$ dim6top } & \multicolumn{2}{|c|}{ dim6top $\rightarrow$ SMEFTsim } \\
\hline $\mathcal{L}_{6}^{(6)}$ & $\begin{array}{l}\text { - ctWRe } \\
\text { - ctWIm } \\
\text {-ctWRe } c_{\theta}+\operatorname{ctBRe} s_{\theta} \\
\text {-ctWIm } c_{\theta}+\operatorname{ctBIm} s_{\theta}\end{array}$ & $\begin{array}{l}\operatorname{ctW} \\
\operatorname{ctWI} \\
\operatorname{ctZ} \\
\operatorname{ctZI}\end{array}$ & $\begin{array}{l}-\operatorname{ctW} \\
-\operatorname{ctWI} \\
\operatorname{ctZ} / s_{\theta}-\operatorname{ctW} / t_{\theta} \\
\operatorname{ctZI} / s_{\theta}-\operatorname{ctWI} / t_{\theta}\end{array}$ & $\begin{array}{l}\text { ctWRe } \\
\text { ctWIm } \\
\text { ctBRe } \\
\text { ctBIm }\end{array}$ \\
\hline $\mathcal{L}_{6}^{(7)}$ & $\begin{array}{l}\text { cHQ3 } \\
\text { cHQ1 - cHQ3 }\end{array}$ & & $\begin{array}{l}\text { cpQ3 + } \mathrm{cpQM} \\
\mathrm{cpQ3}\end{array}$ & $\begin{array}{l}\text { cHQ1 } \\
\text { cHQ3 }\end{array}$ \\
\hline $\mathcal{L}_{6}^{(8 a)}$ & $\begin{array}{l}\text { cQ11 [pp] - cQ13 [pp] } \\
\text { cQ13 [pp] }\end{array}$ & $\begin{array}{l}\text { cQ1M [p] } \\
\text { cQ13 [p] }\end{array}$ & $\begin{array}{l}\text { cQ13 }[\mathrm{p}]+\mathrm{cQ1M}[\mathrm{p}] \\
\mathrm{cQ13}[\mathrm{p}]\end{array}$ & 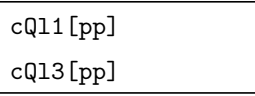 \\
\hline $\mathcal{L}_{6}^{(8 b)}$ & $\begin{array}{l}\frac{1}{3} \text { cutbd1Re }+\frac{4}{9} \text { cutbd8Re } \\
\frac{1}{3} \text { cutbd1Im }+\frac{4}{9} \text { cutbd8Im } \\
2 \text { cutbd1Re }-\frac{1}{3} \text { cutbd8Re } \\
2 \text { cutbd1Im }-\frac{1}{3} \text { cutbd8Im }\end{array}$ & $\begin{array}{lll}\text { yu[r] } & \text { yd [s] } & \text { cbtud1 } \\
\text { yu[r] } & \text { yd[s] } & \text { cbtud1I } \\
\text { yu[r] } & \text { yd[s] } & \text { cbtud8 } \\
\text { yu[r] } & \text { yd[s] } & \text { cbtud8I } \\
\end{array}$ & $\begin{array}{l}\frac{1}{3} \text { cbtud1 }+\frac{4}{9} \text { cbtud8 } \\
\frac{1}{3} \text { cbtud1I }+\frac{4}{9} \text { cbtud8I } \\
2 \text { cbtud } 1-\frac{1}{3} \text { cbtud8 } \\
2 \text { cbtud1I }-\frac{1}{3} \text { cbtud8I } \\
\end{array}$ & $\begin{array}{ll}\frac{1}{\mathrm{yu}[\mathrm{r}] \mathrm{yd}[\mathrm{s}]} & \text { cutbd1Re } \\
\frac{1}{\mathrm{yu}[\mathrm{r}] \mathrm{yd}[\mathrm{s}]} & \text { cutbd1Im } \\
\frac{1}{\mathrm{yu}[\mathrm{r}] \mathrm{yd}[\mathrm{s}]} & \text { cutbd8Re } \\
\frac{1}{\mathrm{yu}[\mathrm{r}] \mathrm{yd}[\mathrm{s}]} & \text { cutbd8Im }\end{array}$ \\
\hline $\mathcal{L}_{6}^{(8 c)}$ & $\begin{array}{l}-\frac{2}{3} \text { cjQtu1Re - } \frac{8}{9} \text { cjQtu8Re } \\
-\frac{2}{3} \text { cjQtu1Im - } \frac{8}{9} \text { cjQtu8Im } \\
-4 \text { cjQtu1Re + } \frac{2}{3} \text { cjQtu8Re } \\
-4 \text { cjQtu1Im + } \frac{2}{3} \text { cjQtu8Im } \\
-\frac{2}{3} \text { cjQbd1Re }-\frac{8}{9} \text { cjQbd8Re } \\
-\frac{2}{3} \text { cjQbd1Im - } \frac{8}{9} \text { cjQbd8Im } \\
-4 \text { cjQbd1Re + } \frac{2}{3} \text { cjQbd8Re } \\
-4 \text { cjQbd1Im + } \frac{2}{3} \text { cjQbd8Im } \\
\end{array}$ & $\begin{array}{ll}\text { yu[r] } & \text { ctQqu1 } \\
\text { yu[r] } & \text { ctQqu1I } \\
\text { yu[r] } & \text { ctQqu8 } \\
\text { yu[r] } & \text { ctQqu8I } \\
\text { yd[r] } & \text { cbQqd1 } \\
\text { yd[r] } & \text { cbQqd1I } \\
\text { yd }[r] & \text { cbQqd8 } \\
\text { yd }[r] & \text { cbQqd8I } \\
\end{array}$ & $\begin{array}{l}-\frac{1}{6} \text { ctQqu1 - } \frac{2}{9} \text { ctQqu8 } \\
-\frac{1}{6} \text { ctQqu1I }-\frac{2}{9} \text { ctQqu8I } \\
- \text { ctQqu1 }+\frac{1}{6} \text { ctQqu8 } \\
- \text { ctQqu1I }+\frac{1}{6} \text { ctQqu8I } \\
-\frac{1}{6} \text { cbQqd1 }-\frac{2}{9} \text { cbQqd8 } \\
-\frac{1}{6} \text { cbQqd1I }-\frac{2}{9} \text { cbQqd8I } \\
- \text { cbQqd1 }+\frac{1}{6} \text { cbQqd8 } \\
- \text { cbQqd1I }+\frac{1}{6} \text { cbQqd8I } \\
\end{array}$ & 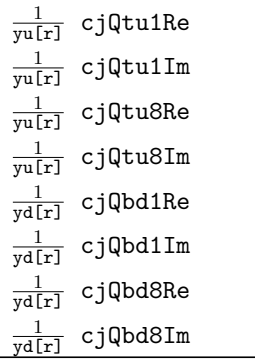 \\
\hline $\mathcal{L}_{6}^{(8 d)}$ & 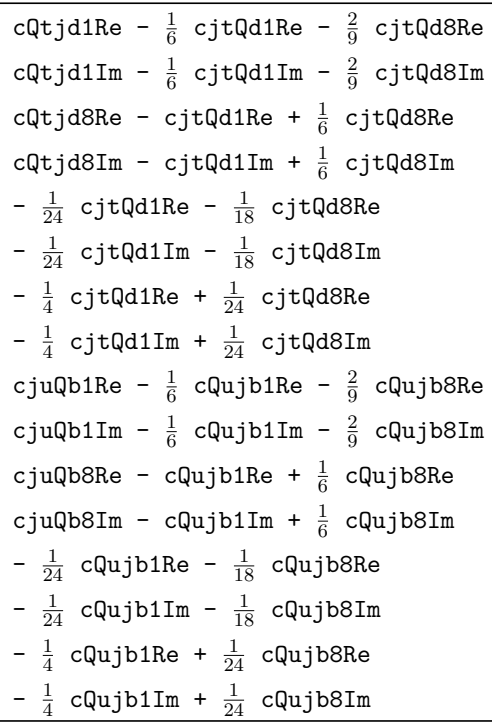 & 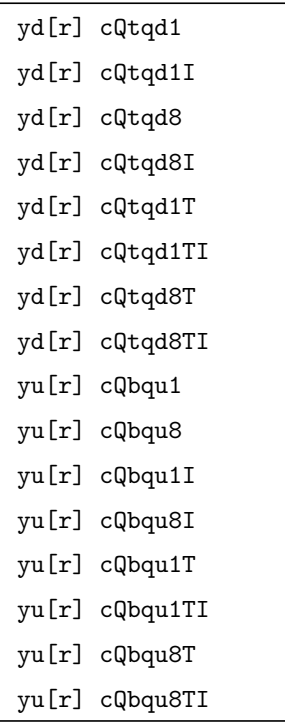 & $\begin{array}{l}\text { cQtqd1 - } 4 \text { cQtqd1T } \\
\text { cQtqd1I - } 4 \text { cQtqd1TI } \\
\text { cQtqd8 - } 4 \text { cQtqd8T } \\
\text { cQtqd8I - } 4 \text { cQtqd8TI } \\
-\frac{8}{3} \text { cQtqd1T - } \frac{32}{9} \text { cQtqd8T } \\
-\frac{8}{3} \text { cQtqd1TI - } \frac{32}{9} \text { cQtqd8TI } \\
-16 \text { cQtqd1T + } \frac{8}{3} \text { cQtqd8T } \\
-16 \text { cQtqd1TI + } \frac{8}{3} \text { cQtqd8TI } \\
\text { cQbqu1 }-4 \text { cQbqu1T } \\
\text { cQbqu1I }-4 \text { cQbqu1TI } \\
\text { cQbqu8 - } 4 \text { cQbqu8T } \\
\text { cQbqu8I - } 4 \text { cQbqu8TI } \\
-\frac{8}{3} \text { cQbqu1T - } \frac{32}{9} \text { cQbqu8T } \\
-\frac{8}{3} \text { cQbqu1TI - } \frac{32}{9} \text { cQbqu8TI } \\
-16 \text { cQbqu1T + } \frac{8}{3} \text { cQbqu8T } \\
-16 \text { cQbqu1TI + } \frac{8}{3} \text { cQbqu8TI }\end{array}$ & 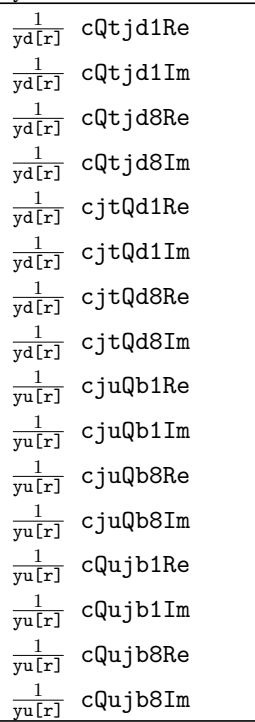 \\
\hline \multicolumn{5}{|c|}{ SMEFTsim topU3I vs dim6top } \\
\hline $\mathcal{L}_{6}^{(8 a)}$ & $\begin{array}{l}\text { cQ11 - cQ13 } \\
\text { cQ13 }\end{array}$ & $\begin{array}{l}\text { cQ1M [p] } \\
\text { cQ13[p] }\end{array}$ & $\begin{array}{l}\text { cQ13[p] + cQ1M[p] } \\
\text { cQ13[p] }\end{array}$ & $\begin{array}{l}\text { CQ11 } \\
\text { CQ13 }\end{array}$ \\
\hline
\end{tabular}

Table 25. Upper panel: conversion table between the SMEFT parameters defined in the top version of SMEFTsim and in dim6top: parameters that require a basis rotation. Lower panel: conversion table between the topU31 version of SMEFTsim and dim6top. Only leptonic coefficients are reported here, as the other parameters behave identically to the top case. Lepton flavor indices take values $\mathrm{p}$ $=\{1,2,3\}$. In the notation $\mathrm{yd}[\mathrm{r}]$, yu $[r]$ etc, the flavor index is that of the associated righthanded light field ( $d$ for yd and $u$ for yu). 


\begin{tabular}{|c|c|c|c|c|c|c|}
\hline \multicolumn{7}{|c|}{ SMEFTsim general vs dim6top } \\
\hline class & \multicolumn{2}{|c|}{ SMEFTsim $\leftrightarrow$ dim6top } & \multicolumn{2}{|c|}{ SMEFTsim $\leftrightarrow$ dim6top } & \multicolumn{2}{|c|}{ SMEFTsim $\leftrightarrow$ dim6top } \\
\hline $\mathcal{L}_{6}^{(5)}$ & cuHRe33 & $\operatorname{ctp}$ & cuHIm33 & $\operatorname{ctpI}$ & & \\
\hline \multirow{2}{*}{$\mathcal{L}_{6}^{(6)}$} & cuGRe33 & $-\operatorname{ctg}$ & cuGIm33 & $-\operatorname{ctGI}$ & & \\
\hline & cdWRe33 & $-\mathrm{cbW}$ & $\operatorname{cdW} \operatorname{Im} 33$ & - cbWI & & \\
\hline \multirow{2}{*}{$\mathcal{L}_{6}^{(7)}$} & cHuRe33 & cpt & cHdRe33 & $\mathrm{cpb}$ & & \\
\hline & cHudRe33 & cptb & cHudIm33 & cptbI & & \\
\hline \multirow{2}{*}{$\mathcal{L}_{6}^{(8 b)}$} & ceu $[\mathrm{pp}] 33$ & $\operatorname{cte}[\mathrm{p}]$ & cuuRe3333 & ctt1 & $\operatorname{cud} 1 \operatorname{Re} 33[\mathrm{rr}]$ & $\operatorname{ctd} 1$ \\
\hline & cud1Re3333 & ctb1 & cud8Re3333 & ctb8 & cud8Re33 [rr] & $\operatorname{ctd} 8$ \\
\hline \multirow{4}{*}{$\mathcal{L}_{6}^{(8 c)}$} & cqu1Re33 $[r r]$ & cQu1 & cqu1Re $[r r] 33$ & ctq1 & cqu1Re3333 & cQt1 \\
\hline & cqu8Re33 $[\mathrm{rr}]$ & cQu8 & cqu8Re $[r r] 33$ & ctq8 & cqu8Re3333 & cQt8 \\
\hline & cqd1Re33 $[r r]$ & cQd1 & cqd1Re3333 & cQb1 & cqd8Re3333 & cQb8 \\
\hline & cqd8Re33 [rr] & cQd8 & cqeRe33 [pp] & $c Q e[p]$ & cluRe $[p p] 33$ & $\operatorname{ctl}[\mathrm{p}]$ \\
\hline \multirow{4}{*}{$\mathcal{L}_{6}^{(8 d)}$} & cledqRe $[p p] 33$ & $\operatorname{cblS}[\mathrm{p}]$ & clequ1Re [pp] 33 & $\operatorname{ctlS}[\mathrm{p}]$ & clequ3Re $[p p] 33$ & $\operatorname{ctlT}[\mathrm{p}]$ \\
\hline & cledqIm [pp] 33 & $\mathrm{cblSI}[\mathrm{p}]$ & clequ1 Im [pp] 33 & $\operatorname{ctlSI}[\mathrm{p}]$ & clequ3Im [pp] 33 & $\operatorname{ctlTI}[\mathrm{p}]$ \\
\hline & cquqd1Re3333 & cQtQb1 & cquqd1Im3333 & cQtQb1I & & \\
\hline & cquqd8Re3333 & cQtQb8 & cquqd8Im3333 & cQtQb8I & & \\
\hline
\end{tabular}

Table 26. Conversion table between the SMEFT parameters defined in the general version of SMEFTsim and in dim6top: parameters with one-to-one conversion. Lepton flavor indices $\mathrm{p}$ take values in $\{1,2,3\}$. Quark flavor indices $r$ take values in $\{1,2\}$. 


\begin{tabular}{|c|c|c|c|c|}
\hline \multicolumn{5}{|c|}{ SMEFTsim general vs dim6top } \\
\hline class & SMEFTsim $\rightarrow$ dim6top & & dim6top $\rightarrow$ SM & \\
\hline $\mathcal{L}_{6}^{(6)}$ & $\begin{array}{l}\text { - cuWRe33 } \\
\text { - cuWIm33 } \\
\text { - cuWRe33 } c_{\theta}+\text { cuBRe33 } s_{\theta} \\
\text { - cuWIm33 } c_{\theta}+\text { cuBIm33 } s_{\theta}\end{array}$ & $\begin{array}{l}\operatorname{ctW} \\
\operatorname{ctWI} \\
\operatorname{ctZ} \\
\operatorname{ctZI}\end{array}$ & $\begin{array}{l}-\operatorname{ctW} \\
-\operatorname{ctWI} \\
\operatorname{ctZ} / s_{\theta}-\operatorname{ctW} / t_{\theta} \\
\operatorname{ctZI} / s_{\theta}-\operatorname{ctWI} / t_{\theta}\end{array}$ & $\begin{array}{l}\text { cuWRe33 } \\
\text { cuWIm33 } \\
\text { cuBRe33 } \\
\text { cuBIm33 }\end{array}$ \\
\hline $\mathcal{L}_{6}^{(7)}$ & $\begin{array}{l}\text { cHq3Re33 } \\
\text { cHq1Re33 - cHq3Re33 }\end{array}$ & $\begin{array}{l}\text { cpQ3 } \\
\text { cpQM }\end{array}$ & $\begin{array}{l}\mathrm{cpQ3}+\mathrm{cpQM} \\
\mathrm{cpQ3}\end{array}$ & $\begin{array}{l}\text { cHq1Re33 } \\
\text { cHq3Re33 }\end{array}$ \\
\hline $\mathcal{L}_{6}^{(8 a)}$ & 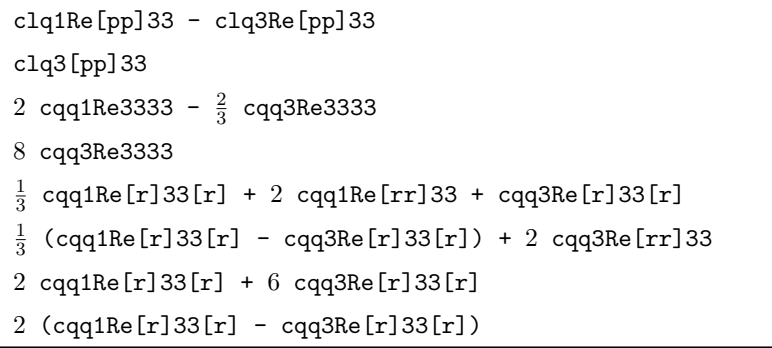 & $\begin{array}{l}\text { cQ1M [p] } \\
\text { cQ13 [p] } \\
\text { cQQ1 } \\
\text { cQQ8 } \\
\text { cQq11 } \\
\text { cQq13 } \\
\text { cQq83 }\end{array}$ & $\begin{array}{l}c Q 13[\mathrm{p}]+\mathrm{cQ} 1 \mathrm{M}[\mathrm{p}] \\
\mathrm{cQ13}[\mathrm{p}] \\
\frac{1}{2} \mathrm{cQQ1}+\frac{1}{24} \mathrm{cQQ8} \\
\frac{1}{8} \mathrm{cQQ8} \\
\frac{1}{2} \mathrm{cQq11}-\frac{1}{12} \mathrm{cQq81} \\
\frac{1}{8} \mathrm{cQq81}+\frac{3}{8} \mathrm{cQq83} \\
\frac{1}{2} \mathrm{cQq13}-\frac{1}{12} \mathrm{cQq83} \\
\frac{1}{8}(\mathrm{cQq} 81-\mathrm{cQq83})\end{array}$ & $\begin{array}{l}\text { clq1Re }[\mathrm{pp}] 33 \\
\text { clq3Re }[\mathrm{pp}] 33 \\
\text { cqq1Re3333 } \\
\text { cqq3Re3333 } \\
\text { cqq1Re }[\mathrm{rr}] 33 \\
\text { cqq1Re }[\mathrm{r}] 33[\mathrm{r}] \\
\text { cqq3Re }[\mathrm{rr}] 33 \\
\text { cqq3Re }[\mathrm{r}] 33[\mathrm{r}]\end{array}$ \\
\hline & $\begin{array}{l}\frac{2}{3} \operatorname{cuuRe}[r] 33[r]+2 \text { cuuRe }[r r] 33 \\
4 \text { cuuRe }[r] 33[r] \\
\frac{1}{3} \text { cud1Re }[r] 33[r]+\frac{4}{9} \text { cud8Re }[r] 33[r] \\
\frac{1}{3} \operatorname{cud} 1 \operatorname{Im}[r] 33[r]+\frac{4}{9} \operatorname{cud} 8 \operatorname{Im}[r] 33[r] \\
2 \operatorname{cud} 1 \operatorname{Re}[r] 33[r]-\frac{1}{3} \operatorname{cud} 8 R e[r] 33[r] \\
2 \operatorname{cud} 1 \operatorname{Im}[r] 33[r]-\frac{1}{3} \operatorname{cud} 8 \operatorname{Im}[r] 33[r]\end{array}$ & $\begin{array}{l}\text { ctu1 } \\
\text { ctu8 } \\
\text { cbtud1 } \\
\text { cbtud1I } \\
\text { cbtud8 } \\
\text { cbtud8I }\end{array}$ & $\begin{array}{l}\frac{1}{2} \text { ctu1 }-\frac{1}{12} \text { ctu } 8 \\
\frac{1}{4} \text { ctu8 } \\
\frac{1}{3} \text { cbtud } 1+\frac{4}{9} \text { cbtud8 } \\
\frac{1}{3} \text { cbtud1I }+\frac{4}{9} \text { cbtud8I } \\
2 \text { cbtud1 }-\frac{1}{3} \text { cbtud8 } \\
2 \text { cbtud1I }-\frac{1}{3} \text { cbtud8I }\end{array}$ & $\begin{array}{l}\text { cuuRe }[r r] 33 \\
\text { cuuRe }[r] 33[r] \\
\text { cud1Re }[r] 33[r] \\
\text { cud1 } \operatorname{Im}[r] 33[r] \\
\text { cud8Re }[r] 33[r] \\
\text { cud8Im }[r] 33[r]\end{array}$ \\
\hline $\mathcal{L}_{6}^{(8 c)}$ & $\begin{array}{l}-\frac{2}{3} \text { cqu1Re }[r] 33[r]-\frac{8}{9} \text { cqu8Re }[r] 33[r] \\
-\frac{2}{3} \operatorname{cqu} 1 \operatorname{Im}[r] 33[r]-\frac{8}{9} \text { cqu8Im }[r] 33[r] \\
-4 \operatorname{cqu} 1 \operatorname{Re}[r] 33[r]+\frac{2}{3} \text { cqu8Re }[r] 33[r] \\
-4 \operatorname{cqu} 1 \operatorname{Im}[r] 33[r]+\frac{2}{3} \text { cqu8Im }[r] 33[r] \\
-\frac{2}{3} \operatorname{cqd1Re}[r] 33[r]-\frac{8}{9} \operatorname{cqd} 8 R e[r] 33[r] \\
-\frac{2}{3} \operatorname{cqd} 1 \operatorname{Im}[r] 33[r]-\frac{8}{9} \operatorname{cqd} 8 \operatorname{Im}[r] 33[r] \\
-4 \operatorname{cqd} 1 \operatorname{Re}[r] 33[r]+\frac{2}{3} \operatorname{cqd} 8 R e[r] 33[r] \\
-4 \operatorname{cqd} 1 \operatorname{Im}[r] 33[r]+\frac{2}{3} \operatorname{cqd} 8 \operatorname{Im}[r] 33[r]\end{array}$ & $\begin{array}{l}\text { ctQqu1 } \\
\text { ctQqu1I } \\
\text { ctQqu8 } \\
\text { ctQqu8I } \\
\text { cbQqd1 } \\
\text { cbQqd1I } \\
\text { cbQqd8 } \\
\text { cbQqd8I }\end{array}$ & $\begin{array}{l}-\frac{1}{6} \text { ctQqu1 - } \frac{2}{9} \text { ctQqu8 } \\
-\frac{1}{6} \text { ctQqu1I - } \frac{2}{9} \text { ctQqu8I } \\
\text { - ctQqu1 + } \frac{1}{6} \text { ctQqu8 } \\
\text { - ctQqu1I + } \frac{1}{6} \text { ctQqu8I } \\
-\frac{1}{6} \text { cbQqd1 - } \frac{2}{9} \text { cbQqd8 } \\
-\frac{1}{6} \text { cbQqd1I }-\frac{2}{9} \text { cbQqd8I } \\
- \text { cbQqd1 }+\frac{1}{6} \text { cbQqd8 } \\
\text { - cbQqd1I }+\frac{1}{6} \text { cbQqd8I }\end{array}$ & $\begin{array}{l}\text { cqu1Re }[r] 33[r] \\
\text { cqu1Im }[r] 33[r] \\
\text { cqu8Re }[r] 33[r] \\
\text { cqu8Im }[r] 33[r] \\
\text { cqd1Re }[r] 33[r] \\
\text { cqd1Im }[r] 33[r] \\
\text { cqd8Re }[r] 33[r] \\
\text { cqd8Im }[r] 33[r]\end{array}$ \\
\hline $\mathcal{L}_{6}^{(8 d)}$ & 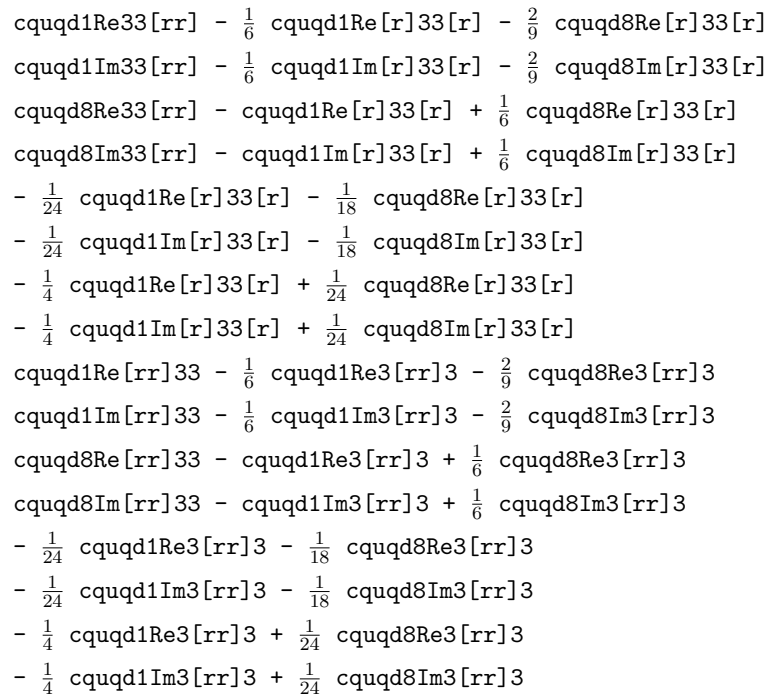 & $\begin{array}{l}\text { cQtqd1 } \\
\text { cQtqd1I } \\
\text { cQtqd8 } \\
\text { cQtqd8I } \\
\text { cQtqd1T } \\
\text { cQtqd1TI } \\
\text { cQtqd8T } \\
\text { cQtqd8TI } \\
\text { cQbqu1 } \\
\text { cQbqu8 } \\
\text { cQbqu1I } \\
\text { cQbqu8I } \\
\text { cQbqu1T } \\
\text { cQbqu1TI } \\
\text { cQbqu8T } \\
\text { cQbqu8TI }\end{array}$ & $\begin{array}{l}\text { cQtqd1 - } 4 \text { cQtqd1T } \\
\text { cQtqd1I - } 4 \text { cQtqd1TI } \\
\text { cQtqd8 - } 4 \text { cQtqd8T } \\
\text { cQtqd8I - } 4 \text { cQtqd8TI } \\
-\frac{8}{3} \text { cQtqd1T - } \frac{32}{9} \text { cQtqd8T } \\
-\frac{8}{3} \text { cQtqd1TI - } \frac{32}{9} \text { cQtqd8TI } \\
-16 \text { cQtqd1T + } \frac{8}{3} \text { cQtqd8T } \\
-16 \text { cQtqd1TI + } \frac{8}{3} \text { cQtqd8TI } \\
\text { cQbqu1 - } 4 \text { cQbqu1T } \\
\text { cQbqu1I - } 4 \text { cQbqu1TI } \\
\text { cQbqu8 - } 4 \text { cQbqu8T } \\
\text { cQbqu8I - } 4 \text { cQbqu8TI } \\
-\frac{8}{3} \text { cQbqu1T - } \frac{32}{9} \text { cQbqu8T } \\
-\frac{8}{3} \text { cQbqu1TI - } \frac{32}{9} \text { cQbqu8TI } \\
-16 \text { cQbqu1T + } \frac{8}{3} \text { cQbqu8T } \\
-16 \text { cQbqu1TI + } \frac{8}{3} \text { cQbqu8TI }\end{array}$ & $\begin{array}{l}\text { cquqd8Im }[r] 33[r] \\
\text { cquqd1Re }[r r] 33 \\
\text { cquqd1 Im }[r r] 33 \\
\text { cquqd8Re }[r r] 33 \\
\text { cquqd8Im }[r r] 33 \\
\text { cquqd1Re3 }[r r] 3 \\
\text { cquqd1Im3 }[r r] 3 \\
\text { cquqd8Re3 }[r r] 3 \\
\text { cquqd8Im3 }[r r] 3\end{array}$ \\
\hline
\end{tabular}

Table 27. Conversion table between the SMEFT parameters defined in the general version of SMEFTsim and in dim6top: parameters that require a basis rotation. Lepton flavor indices $p$ take values in $\{1,2,3\}$. Quark flavor indices $r$ take values in $\{1,2\}$. 


\begin{tabular}{|c|c|c|c|c|c|c|}
\hline \multicolumn{7}{|c|}{ SMEFTsim top vs SMEFT@NLO } \\
\hline class & \multicolumn{2}{|c|}{ SMEFTsim $\leftrightarrow$ SMEFT@NLO } & \multicolumn{2}{|c|}{ SMEFTsim $\leftrightarrow$ SMEFT@NLO } & \multicolumn{2}{|c|}{ SMEFTsim $\leftrightarrow$ SMEFT@NLO } \\
\hline $\mathcal{L}_{6}^{(1)}$ & cG & - gs cG & $\mathrm{cW}$ & - cWWW & & \\
\hline $\mathcal{L}_{6}^{(2,3)}$ & $\mathrm{CH}$ & $\mathrm{cp}$ & cHbox & cdp & $\mathrm{cHDD}$ & $\mathrm{cpDC}$ \\
\hline $\mathcal{L}_{6}^{(4)}$ & $\begin{array}{l}\mathrm{cHG} \\
\mathrm{cHWB}\end{array}$ & $\begin{array}{l}\text { cpG } \\
\text { cpWB }\end{array}$ & $\mathrm{cHW}$ & $\mathrm{cpW}$ & $\mathrm{cHB}$ & $\mathrm{cpBB}$ \\
\hline $\mathcal{L}_{6}^{(5)}$ & ctHRe & $\operatorname{ctp}$ & & & & \\
\hline $\mathcal{L}_{6}^{(6)}$ & ctGRe & - gs ctG & & & & \\
\hline $\mathcal{L}_{6}^{(7)}$ & $\begin{array}{l}\text { cHl1 [pp] } \\
\text { cHe11 } \\
\text { cHu }\end{array}$ & $\begin{array}{l}\mathrm{cpl}[\mathrm{p}] \\
\mathrm{cpe} \\
\mathrm{cpu}\end{array}$ & $\begin{array}{l}\mathrm{cH} 13[\mathrm{pp}] \\
\mathrm{cHe} 22 \\
\mathrm{cHt}\end{array}$ & $\begin{array}{l}\text { c3pl [p] } \\
\text { cpmu } \\
\text { cpt }\end{array}$ & $\begin{array}{l}\mathrm{cHd}=\mathrm{cHbq} \\
\mathrm{cHe} 33\end{array}$ & $\begin{array}{l}\text { cpd } \\
\text { cpta }\end{array}$ \\
\hline $\mathcal{L}_{6}^{(8 a)}$ & $\begin{array}{l}\text { cQj11 } \\
\text { cQj38 } \\
\text { cll [pppp] }\end{array}$ & $\begin{array}{l}\text { cQq11 } \\
\text { cQq83 } \\
\text { cll [pppp] }\end{array}$ & $\begin{array}{l}\text { cQj18 } \\
\text { cQQ1 } \\
\text { cll [pprr] }\end{array}$ & $\begin{array}{l}\text { cQq81 } \\
\frac{1}{2} \text { cQQ1 } \\
2 \text { cll [pprr] }\end{array}$ & $\begin{array}{l}\text { cQj31 } \\
\text { cQQ8 } \\
\text { cll [prrp] }\end{array}$ & $\begin{array}{l}\text { cQq13 } \\
\frac{1}{2} \text { cQQ8 } \\
2 \text { cll [prrp] }\end{array}$ \\
\hline $\mathcal{L}_{6}^{(8 b)}$ & $\begin{array}{l}\text { cte }[\mathrm{pp}] \\
\text { ctt }\end{array}$ & $\begin{array}{l}\operatorname{cte}[\mathrm{p}] \\
\operatorname{ctt} 1\end{array}$ & $\begin{array}{l}\operatorname{ctu} 1 \\
\operatorname{ctd} 1=\operatorname{ctb} 1\end{array}$ & $\begin{array}{l}\operatorname{ctu} 1 \\
\operatorname{ctd} 1\end{array}$ & $\begin{array}{l}\operatorname{ctu} 8 \\
\operatorname{ctd} 8=\operatorname{ctb} 8\end{array}$ & $\begin{array}{l}\text { ctu8 } \\
\operatorname{ctd} 8\end{array}$ \\
\hline $\mathcal{L}_{6}^{(8 c)}$ & $\begin{array}{l}\text { ct1 [pp] } \\
\text { cQt1 } \\
\text { cQu1 } \\
\text { cQu8 }\end{array}$ & $\begin{array}{l}\text { ctl [p] } \\
\text { cQt1 } \\
\text { cQu1 } \\
\text { cQu8 }\end{array}$ & $\begin{array}{l}\text { cQe [pp] } \\
\text { cQt8 } \\
\text { cQd1 = cQb1 } \\
\text { cQd8 = cQb8 }\end{array}$ & $\begin{array}{l}\text { cQe [p] } \\
\text { cQt8 } \\
\text { cQd1 } \\
\text { cQd8 }\end{array}$ & $\begin{array}{l}\operatorname{ctj} 1 \\
\operatorname{ctj} 8\end{array}$ & $\begin{array}{l}\operatorname{ctq} 1 \\
\operatorname{ctq} 8\end{array}$ \\
\hline $\mathcal{L}_{6}^{(8 d)}$ & cleQt1Re33 & ctls3 & cleQt3Re33 & ct1T3 & clebQRe33 & cbls3 \\
\hline
\end{tabular}

\begin{tabular}{|c|c|c|c|c|c|c|}
\hline \multicolumn{7}{|c|}{ SMEFTsim topU31 vs SMEFT@NLO } \\
\hline class & \multicolumn{2}{|c|}{ SMEFTsim $\leftrightarrow$ SMEFT@NLO } & \multicolumn{2}{|c|}{ SMEFTsim $\leftrightarrow$ SMEFT@NLO } & \multicolumn{2}{|c|}{ SMEFTsim $\leftrightarrow$ SMEFT@NLO } \\
\hline $\mathcal{L}_{6}^{(7)}$ & $\mathrm{cHe}$ & $\mathrm{cpe}=\mathrm{cpmu}=\mathrm{cpta}$ & $\mathrm{cHl1}$ & $\mathrm{cpl}[\mathrm{p}]$ & $\mathrm{cHl3}$ & c3pl $[\mathrm{p}]$ \\
\hline $\mathcal{L}_{6}^{(8 a)}$ & cll & $\begin{array}{l}\text { cll [pppp] = } \\
\text { cll[pprr] }\end{array}$ & & & & \\
\hline & cll1 & $\begin{array}{l}\text { cll [pppp] = } \\
\text { cll [prrp] }\end{array}$ & & & & \\
\hline $\mathcal{L}_{6}^{(8 b)}$ & cte & $\operatorname{cte}[\mathrm{p}]$ & & & & \\
\hline $\mathcal{L}_{6}^{(8 c)}$ & cQe & $\mathrm{cQe}[\mathrm{p}]$ & ctl & $\operatorname{ctl}[\mathrm{p}]$ & & \\
\hline $\mathcal{L}_{6}^{(8 d)}$ & cleQt1Re33 & yl[3] ctls3 & cleQt3Re33 & $\mathrm{y} 1[3] \operatorname{ctlT3}$ & clebQRe33 & $\mathrm{yl}[3] \mathrm{cbls} 3$ \\
\hline
\end{tabular}

Table 28. Upper panel: conversion table between the SMEFT parameters defined in the top version of SMEFTsim and in SMEFT@NLO v1.0, for parameters that have a one-to-one translation. Lower panel: conversion table between the topU31 version of SMEFTsim and SMEFT@NLO. Only leptonic coefficients are reported, as the other parameters behave identically to the top case. The = sign indicates that all parameters need to be fixed to the same value. Lepton flavor indices $\mathrm{p}$ take values $p, r=\{1,2,3\}$. 


\begin{tabular}{|c|c|c|c|c|}
\hline \multicolumn{5}{|c|}{ SMEFTsim top vs SMEFT@NLO } \\
\hline class & \multicolumn{2}{|c|}{ SMEFTsim $\rightarrow$ SMEFT@NLO } & \multicolumn{2}{|c|}{ SMEFT@NLO $\rightarrow$ SMEFTsim } \\
\hline $\mathcal{L}_{6}^{(6)}$ & $\begin{array}{l}-\operatorname{ctWRe} \\
-\operatorname{ctWRe} c_{\theta}+\operatorname{ctBRe} s_{\theta}\end{array}$ & $\begin{array}{l}\operatorname{ctW} \\
\operatorname{ctZ}\end{array}$ & $\begin{array}{l}-\operatorname{ctW} \\
\operatorname{ctZ} / s_{\theta}-\operatorname{ctW} / t_{\theta}\end{array}$ & $\begin{array}{l}\text { ctWRe } \\
\text { ctBRe }\end{array}$ \\
\hline $\mathcal{L}_{6}^{(7)}$ & $\begin{array}{l}\text { CHQ3 } \\
\text { CHQ1 - }{ }^{-} \mathrm{HQ3} \\
\mathrm{cHj3} \\
\mathrm{cHj} 1-\mathrm{cHj}_{3}\end{array}$ & $\begin{array}{l}\text { cpQ3 } \\
\text { cpQM } \\
\text { cpq3i } \\
\text { cpqMi }\end{array}$ & $\begin{array}{l}\text { cpQ3 + cpQM } \\
\text { cpQ3 } \\
\text { cpq3i + cpqMi } \\
\text { cpq3i }\end{array}$ & $\begin{array}{l}\mathrm{CHQ1} \\
\mathrm{CHQ3} \\
\mathrm{CHj} 1 \\
\mathrm{CHj}^{3}\end{array}$ \\
\hline $\mathcal{L}_{6}^{(8 a)}$ & $\begin{array}{l}\text { cQ11 [pp] - cQ13[pp] } \\
\text { cQ13[pp] }\end{array}$ & $\begin{array}{l}\text { cQ1M [p] } \\
\text { cQ13 [p] }\end{array}$ & $\begin{array}{l}\mathrm{cQ13}[\mathrm{p}]+\mathrm{cQ1M}[\mathrm{p}] \\
\mathrm{cQ13}[\mathrm{p}]\end{array}$ & $\begin{array}{l}\text { cQ11 [pp] } \\
\text { cQ13 [pp] }\end{array}$ \\
\hline \multicolumn{5}{|c|}{ SMEFTsim topU31 vs SMEFT@NLO } \\
\hline $\mathcal{L}_{6}^{(8 a)}$ & $\begin{array}{l}\text { cQ11 - cQ13 } \\
\text { cQ13 }\end{array}$ & $\begin{array}{l}\text { cQ1M [p] } \\
\text { cQ13 [p] }\end{array}$ & $\begin{array}{l}\mathrm{cQ13}[\mathrm{p}]+\mathrm{cQ1M}[\mathrm{p}] \\
\mathrm{cQ13}[\mathrm{p}]\end{array}$ & $\begin{array}{l}\text { cQ11 } \\
\text { cQ13 }\end{array}$ \\
\hline
\end{tabular}

Table 29. Upper panel: conversion table between the SMEFT parameters defined in the top version of SMEFTsim and in SMEFT@NLO v1.0: parameters that require a basis rotation. Lower panel: conversion table between the topU3I version of SMEFTsim and SMEFT@NLO. Only leptonic coefficients are reported here, as the other parameters behave identically to the top case. Lepton flavor indices take values $\mathrm{p}=\{1,2,3\}$. 


\begin{tabular}{|c|c|c|c|c|c|c|}
\hline \multicolumn{7}{|c|}{ SMEFTsim general vs SMEFT@NLO } \\
\hline class & \multicolumn{2}{|c|}{ SMEFTsim $\leftrightarrow$ SMEFT@NLO } & \multicolumn{2}{|c|}{ SMEFTsim $\leftrightarrow$ SMEFT@NLO } & \multicolumn{2}{|c|}{ SMEFTsim $\leftrightarrow$ SMEFT@NLO } \\
\hline $\mathcal{L}_{6}^{(1)}$ & cG & - gs cG & $\mathrm{cW}$ & - cWWW & & \\
\hline $\mathcal{L}_{6}^{(2,3)}$ & $\mathrm{cH}$ & $\mathrm{cp}$ & cHbox & cdp & $\mathrm{cHDD}$ & $\mathrm{cpDC}$ \\
\hline $\mathcal{L}_{6}^{(4)}$ & $\begin{array}{l}\mathrm{cHG} \\
\mathrm{cHWB}\end{array}$ & $\begin{array}{l}\mathrm{cpG} \\
\mathrm{cpWB}\end{array}$ & $\mathrm{cHW}$ & $\mathrm{cpW}$ & $\mathrm{CHB}$ & $\mathrm{cpBB}$ \\
\hline $\mathcal{L}_{6}^{(5)}$ & cuHRe33 & $\operatorname{ctp}$ & & & & \\
\hline $\mathcal{L}_{6}^{(6)}$ & cuGRe33 & - gs ctG & & & & \\
\hline $\mathcal{L}_{6}^{(7)}$ & $\begin{array}{l}\text { cHl1Re }[\mathrm{pp}] \\
\text { cHeRe11 } \\
\text { cHuRe }[\mathrm{rr}]\end{array}$ & $\begin{array}{l}\text { cpl [p] } \\
\text { cpe } \\
\text { cpu }\end{array}$ & $\begin{array}{l}\text { cHl3Re [pp] } \\
\text { cHe2Re2 } \\
\text { cHuRe33 }\end{array}$ & $\begin{array}{l}\text { c3pl [p] } \\
\text { cpmu } \\
\text { cpt }\end{array}$ & $\begin{array}{l}\text { cHdRe }[r r]=\text { cHdRe33 } \\
\text { cHeRe33 }\end{array}$ & $\begin{array}{l}\text { cpd } \\
\text { cpta }\end{array}$ \\
\hline $\mathcal{L}_{6}^{(8 a)}$ & cllRe [prst] & cll [prst] & & & & \\
\hline $\mathcal{L}_{6}^{(8 b)}$ & ceuRe $[p p] 33$ & $\operatorname{cte}[\mathrm{p}]$ & cuuRe3333 & $\operatorname{ctt} 1$ & $\begin{array}{l}\text { cud1Re33 }[\mathrm{rr}]=\text { cud1Re3333 } \\
\text { cud8Re33 }[\mathrm{rr}]=\text { cud8Re3333 }\end{array}$ & $\begin{array}{l}\operatorname{ctd} 1 \\
\operatorname{ctd} 8\end{array}$ \\
\hline $\mathcal{L}_{6}^{(8 c)}$ & $\begin{array}{l}\text { cluRe }[\mathrm{pp}] 33 \\
\text { cqu1Re3333 } \\
\text { cqu1Re }[\mathrm{rr}] 33 \\
\text { cqu8Re }[\mathrm{rr}] 33\end{array}$ & $\begin{array}{l}\operatorname{ctl}[\mathrm{p}] \\
\operatorname{cQt1} \\
\operatorname{ctq} 1 \\
\operatorname{ctq} 8\end{array}$ & $\begin{array}{l}\text { cqeRe33 }[\mathrm{pp}] \\
\text { cqu8Re3333 } \\
\text { cqu1Re33 }[\mathrm{rr}] \\
\text { cqu8Re33[rr] }\end{array}$ & $\begin{array}{l}\text { cQe [p] } \\
\text { cQt8 } \\
\text { cQu1 } \\
\text { cQu8 }\end{array}$ & $\begin{array}{l}\text { cqd1Re33 }[r r]=\text { cqd1Re3333 } \\
\text { cqd8Re33 }[r r]=\text { cqd8Re3333 }\end{array}$ & $\begin{array}{l}\text { cQd1 } \\
\text { cQd8 }\end{array}$ \\
\hline $\mathcal{L}_{6}^{(8 d)}$ & clequ1Re3333 & ctls3 & clequ3Re3333 & ctlT3 & cledqRe3333 & cbls3 \\
\hline
\end{tabular}

Table 30. Conversion table between the SMEFT parameters defined in the general version of SMEFTsim and in SMEFT@NLO v1.0: set of parameters with one-to-one conversion. Lepton flavor indices $\mathrm{p}$ take values in $\{1,2,3\}$. Quark indices $r$ take values in $\{1,2\}$. 


\begin{tabular}{|c|c|c|c|c|}
\hline \multicolumn{5}{|c|}{ SMEFTsim general vs SMEFT@NLO } \\
\hline class & SMEFTsim $\rightarrow$ SMEFT@NLO & & SMEFT@NLO $\rightarrow$ & SMEFTsim \\
\hline $\mathcal{L}_{6}^{(6)}$ & $\begin{array}{l}\text { - cuWRe33 } \\
\text { - cuWRe33 } c_{\theta}+\text { cuBRe33 } s_{\theta}\end{array}$ & $\begin{array}{l}\operatorname{ctW} \\
\operatorname{ctZ}\end{array}$ & $\begin{array}{l}-\operatorname{ctW} \\
\operatorname{ctZ} / s_{\theta}-\operatorname{ctW} / t_{\theta}\end{array}$ & $\begin{array}{l}\text { cuWRe33 } \\
\text { cuBRe33 }\end{array}$ \\
\hline $\mathcal{L}_{6}^{(7)}$ & $\begin{array}{l}\text { cHq3Re33 } \\
\text { cHq1Re33 - cHq3Re33 } \\
\text { cHq3Re }[r r] \\
\text { cHq1Re }[r r]-c H q 3 R e[r r]\end{array}$ & $\begin{array}{l}\text { cpQ3 } \\
\text { cpQM } \\
\text { cpq3i } \\
\text { cpqMi }\end{array}$ & $\begin{array}{l}\mathrm{cpQ3}+\mathrm{cpQM} \\
\mathrm{cpQ3} \\
\mathrm{cpq3i}+\mathrm{cpqMi} \\
\mathrm{cpq3i}\end{array}$ & $\begin{array}{l}\text { cHq1Re33 } \\
\text { cHq3Re33 } \\
\text { cHq1Re }[r r] \\
\text { cHq3Re }[r r]\end{array}$ \\
\hline $\mathcal{L}_{6}^{(8 a)}$ & $\begin{array}{l}\text { clq1Re }[\mathrm{pp}] 33-\mathrm{clq} 3 \operatorname{Re}[\mathrm{pp}] 33 \\
\text { clq3 }[\mathrm{pp}] 33 \\
2 \text { cqq1Re3333- } \frac{2}{3} \text { cqq3Re3333 } \\
8 \text { cqq3Re3333 } \\
\frac{1}{3} \text { cqq1Re}[r] 33[\mathrm{r}]+2 \mathrm{cqq} 1 \operatorname{Re}[\mathrm{rr}] 33+\mathrm{cqq} 3 \operatorname{Re}[\mathrm{r}] 33[\mathrm{r}] \\
\frac{1}{3}(\mathrm{cqq} 1 \operatorname{Re}[\mathrm{r}] 33[\mathrm{r}]-\mathrm{cqq} 3 \operatorname{Re}[\mathrm{r}] 33[\mathrm{r}])+2 \mathrm{cqq} 3 \operatorname{Re}[\mathrm{rr}] 33 \\
2 \mathrm{cqq} 1 \operatorname{Re}[\mathrm{r}] 33[\mathrm{r}]+6 \mathrm{cqq} 3 \operatorname{Re}[\mathrm{r}] 33[\mathrm{r}] \\
2(\mathrm{cqq} 1 \operatorname{Re}[\mathrm{r}] 33[\mathrm{r}]-\mathrm{cqq} 3 \operatorname{Re}[\mathrm{r}] 33[\mathrm{r}]) \\
\end{array}$ & $\begin{array}{l}\text { cQ1M [p] } \\
\text { cQ13 [p] } \\
\text { cQQ1 } \\
\text { cQQ8 } \\
\text { cQq11 } \\
\text { cQq81 } \\
\text { cQq83 }\end{array}$ & $\begin{array}{l}\mathrm{cQ13}[\mathrm{p}]+\mathrm{cQ1M}[\mathrm{p}] \\
\mathrm{cQ13}[\mathrm{p}] \\
\frac{1}{2} \mathrm{cQQ1}+\frac{1}{24} \mathrm{cQQ8} \\
\frac{1}{8} \mathrm{cQQ8} \\
\frac{1}{2} \mathrm{cQq11}-\frac{1}{12} \mathrm{cQq81} \\
\frac{1}{8} \mathrm{cQq81}+\frac{3}{8} \mathrm{cQq83} \\
\frac{1}{2} \mathrm{cQq13}-\frac{1}{12} \mathrm{cQq83} \\
\frac{1}{8}(\mathrm{cQq} 81-\mathrm{cQq83})\end{array}$ & $\begin{array}{l}\text { clq1Re }[\mathrm{pp}] 33 \\
\text { clq3Re }[\mathrm{pp}] 33 \\
\text { cqq1Re3333 } \\
\text { cqq3Re3333 } \\
\text { cqq1Re }[\mathrm{rr}] 33 \\
\text { cqq1Re }[\mathrm{r}] 33[\mathrm{r}] \\
\text { cqq3Re }[\mathrm{rr}] 33 \\
\text { cqq3Re }[\mathrm{r}] 33[\mathrm{r}]\end{array}$ \\
\hline $\mathcal{L}_{6}^{(8 b)}$ & $\begin{array}{l}\frac{2}{3} \text { cuuRe }[r] 33[r]+2 \text { cuuRe }[r r] 33 \\
4 \text { cuuRe }[r] 33[r]\end{array}$ & $\begin{array}{l}\text { ctu1 } \\
\text { ctu8 }\end{array}$ & $\begin{array}{l}\frac{1}{2} \operatorname{ctu} 1-\frac{1}{12} \operatorname{ctu} 8 \\
\frac{1}{4} \text { ctu8 }\end{array}$ & $\begin{array}{l}\text { cuuRe }[r r] 33 \\
\text { cuuRe }[r] 33[r]\end{array}$ \\
\hline
\end{tabular}

Table 31. Conversion table between the SMEFT parameters defined in the general version of SMEFTsim and in SMEFT@NLO v1.0: parameters that require a basis rotation. Lepton flavor indices $\mathrm{p}$ take values in $\{1,2,3\}$. Quark flavor indices $r$ take values in $\{1,2\}$. 


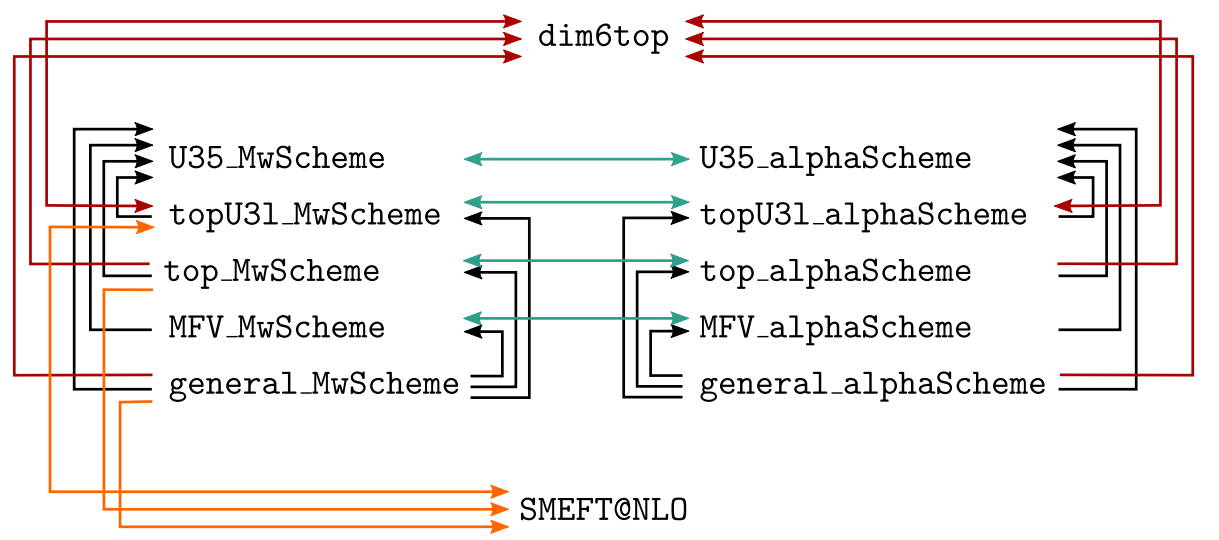

Figure 3. Diagram of the pairwise comparisons performed to validate the UFO models. The arrow indicates the direction in which the SMEFT parameters are mapped.

\section{F Validation of the UFO models}

The 10 UFO models contained in the SMEFTsim package have been validated following the recommendations of ref. [47]: the procedure relies on pairwise comparisons between models, based on the values returned for a set of squared amplitudes. Each comparison is performed with the dedicated MadGraph5_aMC@NLO plugin [123]: given a list of $2 \rightarrow n$ processes and of points in parameter space, the SM squared amplitude $\left|A_{\mathrm{SM}}\right|^{2}$, the pure SM- $\mathcal{L}_{6}$ interference $2 \operatorname{Re} A_{\mathrm{SM}} A_{6}^{*}$ and the quadratic $\mathcal{L}_{6}$ contribution $\left|A_{6}\right|^{2}$ are calculated at one random phasespace point for each process and parameter point. The validation is considered successful if the squared amplitudes evaluated with each model pair agree within a permille. Larger discrepancies are ignored if they do not show a consistent pattern across different processes and the squared amplitude is $<10^{-16}$ for both models.

Figure 3 illustrates diagrammatically the set of comparisons performed: the top, topU31 and general versions of SMEFTsim have been compared to dim6top (version of May 2020) and SMEFT@NLO (both versions of August 2019 and September 2020, only for models with $\left\{m_{W}, m_{Z}, G_{F}\right\}$ scheme). An internal validation was also carried out, comparing models with different flavor assumptions and same input scheme, and vice versa. The arrows in the figure indicate that, in the comparison, the parameters of the first model were mapped onto those of the latter: the flow generally goes towards more restrictive flavor assumptions.

The validation was performed on the processes listed in table 32 , that were chosen so as to probe most effective operators independently. All Wilson coefficients have been included in the comparison, with the exception of those inducing flavor-changing neutral currents.

All fermion masses and Yukawa couplings were retained for internal validation, while only those implemented in dim6top or SMEFT@NLO were included when comparing to these models. CKM mixing has been neglected in all cases.

Linearized propagator corrections have been validated with an analogous procedure, using the processes listed in table 33 and carrying out internal comparisons across models with same inputs and different flavor assumption. Loop-induced SM Higgs couplings have 


\begin{tabular}{|c|c|c|c|c|c|}
\hline $\begin{array}{l}\mathrm{g} g>t \mathrm{t} \\
\mathrm{w}^{+} \mathrm{w}^{-}>\mathrm{a} a \\
\mathrm{~h} \mathrm{w}^{+}>\mathrm{ud} \\
\mathrm{h} \mathrm{a}>\mathrm{b} \mathrm{b} \\
\mathrm{h} \mathrm{a}>\mathrm{e}^{+} \mathrm{e}^{-} \\
\mathrm{t} \mathrm{t}>\mathrm{t} \\
\mathrm{u} \mathrm{u}>\mathrm{cc} \\
\mathrm{e}^{+} \mathrm{e}^{-}>\mathrm{mu}+\mathrm{mu}- \\
\mathrm{e}^{+} \mathrm{ve}>\mathrm{e}^{+} \mathrm{ve} \\
\mathrm{d} \mathrm{d}>\mathrm{e}^{+} \mathrm{e}^{-} \\
\mathrm{t} \mathrm{t}>\mathrm{vm} \mathrm{vm} \\
\mathrm{w}^{+} \mathrm{w}^{-}>\mathrm{a} \mathrm{a} \\
\mathrm{h} \mathrm{h}>\mathrm{h} \mathrm{s}^{-} \\
\mathrm{h} \mathrm{h}>\mathrm{e}^{+} \mathrm{e}^{-} \\
\mathrm{h} \mathrm{h}>\mathrm{w}^{+} \mathrm{w}^{-} \mathrm{a}\end{array}$ & 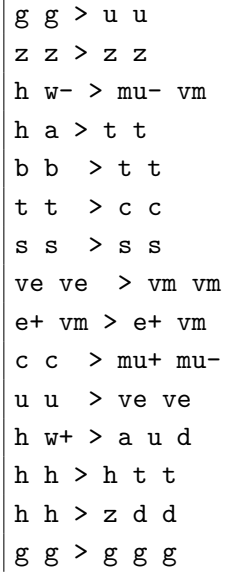 & 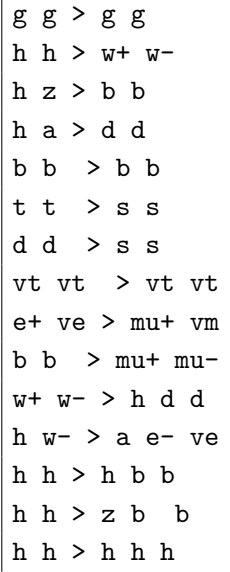 & 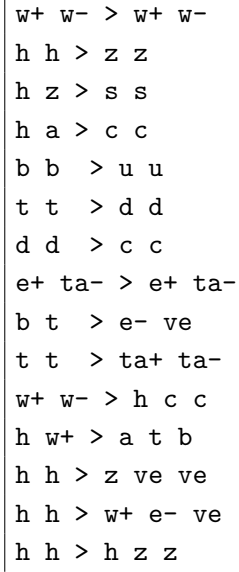 & 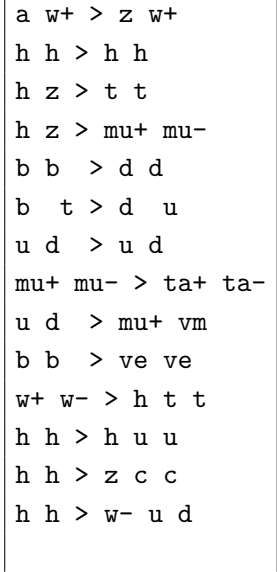 & 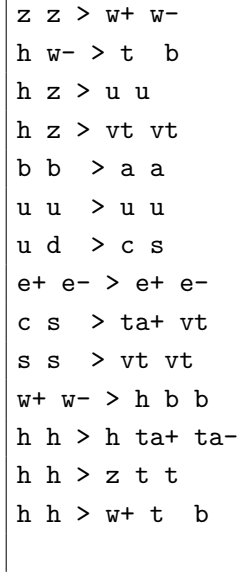 \\
\hline
\end{tabular}

Table 32. Set of processes that have been used to validate the UFO models.

\begin{tabular}{|c|c|c|c|c|c|}
\hline $\begin{array}{l}\mathrm{w}^{+} \mathrm{w}^{-}>\mathrm{w}^{+} \mathrm{w}^{-} \\
\mathrm{h} \mathrm{w}^{+}>\mathrm{ud} \\
\mathrm{b} \mathrm{b}>\mathrm{t} \mathrm{t} \\
\mathrm{d} \mathrm{d}>\mathrm{cc} \\
\mathrm{e}+\mathrm{ve}>\mathrm{e}^{+} \mathrm{ve} \\
\mathrm{b} \mathrm{b}>\mathrm{mu}+\mathrm{mu}- \\
\mathrm{w}^{+} \mathrm{w}^{-}>\mathrm{hcc} \\
\mathrm{h} \mathrm{h}>\mathrm{hta} \mathrm{ta}^{-} \\
\mathrm{h} \mathrm{h}>\mathrm{w}^{-} \mathrm{ud}\end{array}$ & $\begin{array}{l}z \mathrm{z}>\mathrm{w}^{+} \mathrm{w}^{-} \\
\mathrm{h} \mathrm{w}^{-}>\mathrm{mu}-\mathrm{vm} \\
\mathrm{b} \mathrm{b}>\mathrm{b} \mathrm{b} \\
\mathrm{u} d>\mathrm{c} \mathrm{s} \\
\text { e+ vm>e+vm } \\
\mathrm{b} \mathrm{b}>\mathrm{ve} \text { ve } \\
\mathrm{w}^{+} \mathrm{w}^{-}>\mathrm{h} b \mathrm{~b} \\
\mathrm{~h} \mathrm{~h}>\mathrm{h} \mathrm{b} b \\
\mathrm{~h} \mathrm{~h}>\mathrm{w}^{+} \mathrm{w}^{-} \mathrm{a}\end{array}$ & $\begin{array}{l}\mathrm{w}^{+} \mathrm{w}^{-}>\mathrm{a} a \\
\mathrm{~h} \mathrm{z}>\mathrm{s} \mathrm{s} \\
\mathrm{t} \mathrm{t}>\mathrm{t} \mathrm{t} \\
\mathrm{e}+\mathrm{e}->\mathrm{mu}+\mathrm{mu}- \\
\mathrm{e}+\mathrm{ve}>\mathrm{mu}+\mathrm{vm} \\
\mathrm{s} \mathrm{s}>\mathrm{vt} \mathrm{vt} \\
\mathrm{w}^{+} \mathrm{w}^{-}>\mathrm{aaa} \\
\mathrm{h} \mathrm{h}>\mathrm{zcc}\end{array}$ & 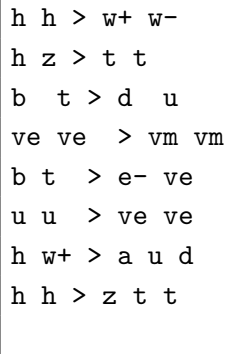 & 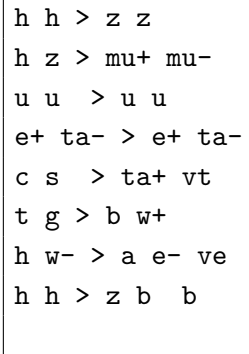 & $\begin{array}{l}\mathrm{h} h>\mathrm{h} \mathrm{h} \\
\mathrm{h} z>\mathrm{vt} \mathrm{vt} \\
\mathrm{s} \mathrm{s}>\mathrm{s} \mathrm{s} \\
\mathrm{e}+\mathrm{e}^{-}>\mathrm{e}^{+} \mathrm{e}^{-} \\
\mathrm{c} \mathrm{c}>\mathrm{mu}+\mathrm{mu}- \\
\mathrm{w}^{+} \mathrm{w}^{-}>\mathrm{hdd} \\
\mathrm{h} \mathrm{w}^{+}>\mathrm{atb} \\
\mathrm{h} \mathrm{h}>\mathrm{w}^{+} \mathrm{e}^{-} \mathrm{ve}\end{array}$ \\
\hline
\end{tabular}

Table 33. Set of processes that have been used to validate linearized propagator corrections implemented in the UFO models.

\begin{tabular}{|c|c|c|c|c|c|}
\hline $\begin{array}{l}\mathrm{g} g>t \mathrm{t} \\
\mathrm{h} \mathrm{a}>\mathrm{b} \mathrm{b} \\
\mathrm{w}^{+} \mathrm{w}^{-}>\mathrm{h} \mathrm{d} d \\
\mathrm{~h} \mathrm{~h}>\mathrm{zdd}\end{array}$ & $\begin{array}{l}\mathrm{g} g>\mathrm{u} u \\
\mathrm{~h} a>d \mathrm{~d} \\
\mathrm{w}^{+} \mathrm{w}^{-}>\mathrm{h} \mathrm{t} \\
\mathrm{h} \mathrm{h}>\mathrm{zb} \mathrm{b}\end{array}$ & $\begin{array}{l}\mathrm{g} g>\mathrm{g} g \\
\mathrm{~h} a>c \mathrm{c} \\
\mathrm{w}^{+} \mathrm{w}^{-}>\mathrm{h} \mathrm{b} b \\
\mathrm{~g} \mathrm{~g}>\mathrm{g} \mathrm{g} \mathrm{g}\end{array}$ & $\begin{array}{l}\mathrm{a} \mathrm{w}^{+}>\mathrm{z} \mathrm{w}^{+} \\
\mathrm{h} \mathrm{a}>\mathrm{e}^{+} \mathrm{e}^{-} \\
\mathrm{w}^{+} \mathrm{w}^{-}>\mathrm{a} \mathrm{a} a \\
\mathrm{~h} \mathrm{~h}>\mathrm{h} \mathrm{h} \mathrm{h}\end{array}$ & $\begin{array}{l}z \mathrm{z}>\mathrm{w}^{+} \mathrm{w}^{-} \\
\mathrm{b} \mathrm{b}>\mathrm{a} a \\
\mathrm{~h} \mathrm{~h}>\mathrm{zcc}\end{array}$ & $\begin{array}{l}\mathrm{w}^{+} \mathrm{w}^{-}>\mathrm{a} a \\
\mathrm{t} \mathrm{t}>\mathrm{za} \\
\mathrm{h} \mathrm{h}>\mathrm{ze} \mathrm{e}^{+} \mathrm{e}^{-}\end{array}$ \\
\hline
\end{tabular}

Table 34. Set of processes that have been used to validate loop-induced SM Higgs couplings implemented in the UFO models.

been validated comparing the SM squared amplitudes at one phase-space point for each of the processes in table 34 with all models.

The output files generated by the MadGraph5_aMC@NLO plugin are available at the github repository. No significant residual differences are present between models. Only one exception was observed: potentially large discrepancies are present between SMEFTsim (both top and general) and dim6top, for the Wilson coefficients cQbqu1T, cQbqu8T, cQtqd1T, cQtqd8T and the associated imaginary parts. These differences have been already noted in the past and are currently not fully understood.

Open Access. This article is distributed under the terms of the Creative Commons Attribution License (CC-BY 4.0), which permits any use, distribution and reproduction in any medium, provided the original author(s) and source are credited. 


\section{References}

[1] I. Brivio, Y. Jiang and M. Trott, The SMEFTsim package, theory and tools, JHEP 12 (2017) 070 [arXiv: 1709.06492] [inSPIRE].

[2] I. Brivio and M. Trott, The Standard Model as an Effective Field Theory, Phys. Rept. 793 (2019) 1 [arXiv: 1706 .08945] [INSPIRE].

[3] G. Passarino, XEFT, the challenging path up the hill: $\operatorname{dim}=6$ and $\operatorname{dim}=8$, arXiv: 1901.04177 [INSPIRE].

[4] A. David and G. Passarino, Use and reuse of SMEFT, arXiv:2009.00127 [InSPIRE].

[5] E. da Silva Almeida, A. Alves, N. Rosa Agostinho, O.J.P. Éboli and M.C. Gonzalez-Garcia, Electroweak Sector Under Scrutiny: A Combined Analysis of LHC and Electroweak Precision Data, Phys. Rev. D 99 (2019) 033001 [arXiv:1812.01009] [InSPIRE].

[6] A. Biekoetter, T. Corbett and T. Plehn, The Gauge-Higgs Legacy of the LHC Run II, SciPost Phys. 6 (2019) 064 [arXiv:1812.07587] [InSPIRE].

[7] N.P. Hartland et al., A Monte Carlo global analysis of the Standard Model Effective Field Theory: the top quark sector, JHEP 04 (2019) 100 [arXiv:1901.05965] [INSPIRE].

[8] S. van Beek, E.R. Nocera, J. Rojo and E. Slade, Constraining the SMEFT with Bayesian reweighting, SciPost Phys. 7 (2019) 070 [arXiv: 1906.05296] [INSPIRE].

[9] I. Brivio et al., O new physics, where art thou? A global search in the top sector, JHEP 02 (2020) 131 [arXiv: 1910.03606] [INSPIRE].

[10] A. Falkowski and D. Straub, Flavourful SMEFT likelihood for Higgs and electroweak data, JHEP 04 (2020) 066 [arXiv: 1911.07866] [inSPIRE].

[11] S. Bißmann, J. Erdmann, C. Grunwald, G. Hiller and K. Kröninger, Correlating uncertainties in global analyses within SMEFT matters, Phys. Rev. D 102 (2020) 115019 [arXiv: 1912.06090] [INSPIRE].

[12] S. Dawson, S. Homiller and S.D. Lane, Putting standard model EFT fits to work, Phys. Rev. D 102 (2020) 055012 [arXiv: 2007.01296] [INSPIRE].

[13] J. Ellis, M. Madigan, K. Mimasu, V. Sanz and T. You, Top, Higgs, Diboson and Electroweak Fit to the Standard Model Effective Field Theory, arXiv:2012.02779 [INSPIRE].

[14] ATLAS collaboration, Interpretations of the combined measurement of Higgs boson production and decay, Tech. Rep. ATLAS-CONF-2020-053 (2020).

[15] J.J. Ethier, R. Gomez-Ambrosio, G. Magni and J. Rojo, SMEFT analysis of vector boson scattering and diboson data from the LHC Run II, arXiv:2101.03180 [INSPIRE].

[16] S. Bißmann, J. Erdmann, C. Grunwald, G. Hiller and K. Kröninger, Constraining top-quark couplings combining top-quark and $\boldsymbol{B}$ decay observables, Eur. Phys. J. C 80 (2020) 136 [arXiv: 1909.13632] [INSPIRE].

[17] R. Aoude, T. Hurth, S. Renner and W. Shepherd, The impact of flavour data on global fits of the MFV SMEFT, JHEP 12 (2020) 113 [arXiv:2003.05432] [INSPIRE].

[18] S. Bißmann, C. Grunwald, G. Hiller and K. Kröninger, Top and Beauty synergies in SMEFT-fits at present and future colliders, arXiv:2012.10456 [INSPIRE].

[19] S. Bruggisser, R. Schäfer, D. van Dyk and S. Westhoff, The Flavor of UV Physics, arXiv:2101.07273 [INSPIRE]. 
[20] J. Aebischer, M. Fael, A. Lenz, M. Spannowsky and J. Virto eds., Computing Tools for the SMEFT, arXiv:1910.11003 [INSPIRE].

[21] A. Falkowski, B. Fuks, K. Mawatari, K. Mimasu, F. Riva and V. Sanz, Rosetta: an operator basis translator for Standard Model effective field theory, Eur. Phys. J. C 75 (2015) 583 [arXiv: 1508.05895] [INSPIRE].

[22] J. Aebischer et al., WCxf: an exchange format for Wilson coefficients beyond the Standard Model, Comput. Phys. Commun. 232 (2018) 71 [arXiv:1712.05298] [INSPIRE].

[23] B. Gripaios and D. Sutherland, DEFT: A program for operators in EFT, JHEP 01 (2019) 128 [arXiv: 1807.07546] [INSPIRE].

[24] J.C. Criado, BasisGen: automatic generation of operator bases, Eur. Phys. J. C 79 (2019) 256 [arXiv: 1901.03501] [INSPIRE].

[25] A. Celis, J. Fuentes-Martin, A. Vicente and J. Virto, DsixTools: The Standard Model Effective Field Theory Toolkit, Eur. Phys. J. C 77 (2017) 405 [arXiv:1704.04504] [INSPIRE].

[26] J.C. Criado, MatchingTools: a Python library for symbolic effective field theory calculations, Comput. Phys. Commun. 227 (2018) 42 [arXiv:1710.06445] [INSPIRE].

[27] J. Aebischer, J. Kumar and D.M. Straub, Wilson: a Python package for the running and matching of Wilson coefficients above and below the electroweak scale, Eur. Phys. J. C 78 (2018) 1026 [arXiv: 1804.05033] [INSPIRE].

[28] S. Das Bakshi, J. Chakrabortty and S.K. Patra, CoDEx: Wilson coefficient calculator connecting SMEFT to UV theory, Eur. Phys. J. C 79 (2019) 21 [arXiv:1808.04403] [INSPIRE].

[29] J. Fuentes-Martin, P. Ruiz-Femenia, A. Vicente and J. Virto, DsixTools 2.0: The Effective Field Theory Toolkit, Eur. Phys. J. C 81 (2021) 167 [arXiv:2010.16341] [InSPIRE].

[30] T. Cohen, X. Lu and Z. Zhang, STrEAMlining EFT Matching, arXiv:2012.07851 [INSPIRE].

[31] J. Fuentes-Martin, M. König, J. Pagès, A.E. Thomsen and F. Wilsch, SuperTracer: A Calculator of Functional Supertraces for One-Loop EFT Matching, arXiv:2012.08506 [INSPIRE].

[32] A. Dedes, W. Materkowska, M. Paraskevas, J. Rosiek and K. Suxho, Feynman rules for the Standard Model Effective Field Theory in $R_{\xi}$-gauges, JHEP 06 (2017) 143 [arXiv: 1704.03888] [INSPIRE].

[33] A. Dedes, M. Paraskevas, J. Rosiek, K. Suxho and L. Trifyllis, SmeftFR - Feynman rules generator for the Standard Model Effective Field Theory, Comput. Phys. Commun. 247 (2020) 106931 [arXiv:1904.03204] [INSPIRE].

[34] T. Corbett, The Feynman rules for the SMEFT in the background field gauge, JHEP 03 (2021) 001 [arXiv : 2010.15852] [inSPIRE].

[35] A. Alloul, B. Fuks and V. Sanz, Phenomenology of the Higgs Effective Lagrangian via FEYNRULES, JHEP 04 (2014) 110 [arXiv:1310.5150] [INSPIRE].

[36] D. Barducci et al., Interpreting top-quark LHC measurements in the standard-model effective field theory, arXiv: 1802.07237 [INSPIRE]. 
[37] C. Degrande, G. Durieux, F. Maltoni, K. Mimasu, E. Vryonidou and C. Zhang, Automated one-loop computations in the SMEFT, arXiv:2008.11743 [INSPIRE].

[38] N. Castro, J. Erdmann, C. Grunwald, K. Kröninger and N.-A. Rosien, EFTfitter - A tool for interpreting measurements in the context of effective field theories, Eur. Phys. J. C 76 (2016) 432 [arXiv: 1605.05585] [INSPIRE].

[39] J. Aebischer, J. Kumar, P. Stangl and D.M. Straub, A Global Likelihood for Precision Constraints and Flavour Anomalies, Eur. Phys. J. C 79 (2019) 509 [arXiv:1810.07698] [INSPIRE].

[40] D.M. Straub, flavio: a Python package for flavour and precision phenomenology in the Standard Model and beyond, arXiv:1810.08132 [INSPIRE].

[41] J. De Blas et al., HEPfit: a code for the combination of indirect and direct constraints on high energy physics models, Eur. Phys. J. C 80 (2020) 456 [arXiv:1910.14012] [InSPIRE].

[42] B. Grzadkowski, M. Iskrzynski, M. Misiak and J. Rosiek, Dimension-Six Terms in the Standard Model Lagrangian, JHEP 10 (2010) 085 [arXiv: 1008.4884] [INSPIRE].

[43] N.D. Christensen and C. Duhr, FeynRules - Feynman rules made easy, Comput. Phys. Commun. 180 (2009) 1614 [arXiv:0806.4194] [INSPIRE].

[44] A. Alloul, N.D. Christensen, C. Degrande, C. Duhr and B. Fuks, FeynRules 2.0 - A complete toolbox for tree-level phenomenology, Comput. Phys. Commun. 185 (2014) 2250 [arXiv: 1310.1921] [INSPIRE].

[45] C. Degrande, C. Duhr, B. Fuks, D. Grellscheid, O. Mattelaer and T. Reiter, UFO - The Universal FeynRules Output, Comput. Phys. Commun. 183 (2012) 1201 [arXiv:1108.2040] [INSPIRE].

[46] J. Alwall et al., The automated computation of tree-level and next-to-leading order differential cross sections, and their matching to parton shower simulations, JHEP $\mathbf{0 7}$ (2014) 079 [arXiv: 1405.0301] [INSPIRE].

[47] F. Maltoni et al., Proposal for the validation of Monte Carlo implementations of the standard model effective field theory, arXiv:1906.12310 [INSPIRE].

[48] R. Alonso, E.E. Jenkins, A.V. Manohar and M. Trott, Renormalization Group Evolution of the Standard Model Dimension Six Operators III: Gauge Coupling Dependence and Phenomenology, JHEP 04 (2014) 159 [arXiv:1312.2014] [INSPIRE].

[49] B. Grinstein and M.B. Wise, Operator analysis for precision electroweak physics, Phys. Lett. B 265 (1991) 326 [INSPIRE].

[50] T. Corbett, O.J.P. Eboli, J. Gonzalez-Fraile and M.C. Gonzalez-Garcia, Robust Determination of the Higgs Couplings: Power to the Data, Phys. Rev. D 87 (2013) 015022 [arXiv:1211.4580] [INSPIRE].

[51] M. Ghezzi, R. Gomez-Ambrosio, G. Passarino and S. Uccirati, NLO Higgs effective field theory and $\kappa$-framework, JHEP 07 (2015) 175 [arXiv: 1505.03706] [INSPIRE].

[52] L. Berthier and M. Trott, Towards consistent Electroweak Precision Data constraints in the SMEFT, JHEP 05 (2015) 024 [arXiv: 1502.02570] [INSPIRE].

[53] R. Gauld, B.D. Pecjak and D.J. Scott, One-loop corrections to $h \rightarrow b \bar{b}$ and $h \rightarrow \tau \bar{\tau}$ decays in the Standard Model Dimension-6 EFT: four-fermion operators and the large-m limit, $^{-}$ JHEP 05 (2016) 080 [arXiv:1512.02508] [INSPIRE]. 
[54] G. Passarino and M. Trott, The Standard Model Effective Field Theory and Next to Leading Order, arXiv: 1610.08356 [INSPIRE].

[55] S. Dawson and P.P. Giardino, Electroweak corrections to Higgs boson decays to $\gamma \gamma$ and $W^{+} W^{-}$in standard model EFT, Phys. Rev. D 98 (2018) 095005 [arXiv:1807.11504] [INSPIRE].

[56] S. Dawson and P.P. Giardino, Higgs decays to $Z Z$ and $Z \gamma$ in the standard model effective field theory: An NLO analysis, Phys. Rev. D 97 (2018) 093003 [arXiv:1801.01136] [INSPIRE].

[57] I. Brivio, T. Corbett and M. Trott, The Higgs width in the SMEFT, JHEP 10 (2019) 056 [arXiv: 1906. 06949] [InSPIRE].

[58] J.M. Cullen, B.D. Pecjak and D.J. Scott, NLO corrections to $h \rightarrow b \bar{b}$ decay in SMEFT, JHEP 08 (2019) 173 [arXiv: 1904.06358] [INSPIRE].

[59] A. Denner and S. Dittmaier, Electroweak Radiative Corrections for Collider Physics, Phys. Rept. 864 (2020) 1 [arXiv: 1912.06823] [INSPIRE].

[60] A. Helset, M. Paraskevas and M. Trott, Gauge fixing the Standard Model Effective Field Theory, Phys. Rev. Lett. 120 (2018) 251801 [arXiv:1803.08001] [InSPIRE].

[61] M. Misiak, M. Paraskevas, J. Rosiek, K. Suxho and B. Zglinicki, Effective Field Theories in $R_{\xi}$ gauges, JHEP 02 (2019) 051 [arXiv:1812.11513] [INSPIRE].

[62] A. Helset, A. Martin and M. Trott, The Geometric Standard Model Effective Field Theory, JHEP 03 (2020) 163 [arXiv:2001.01453] [INSPIRE].

[63] I. Brivio and M. Trott, Scheming in the SMEFT... and a reparameterization invariance!, JHEP 07 (2017) 148 [Addendum ibid. 05 (2018) 136] [arXiv: 1701.06424] [INSPIRE].

[64] L. Wolfenstein, Parametrization of the Kobayashi-Maskawa Matrix, Phys. Rev. Lett. 51 (1983) 1945 [INSPIRE].

[65] R.S. Chivukula and H. Georgi, Composite Technicolor Standard Model, Phys. Lett. B 188 (1987) 99 [INSPIRE].

[66] D.A. Faroughy, G. Isidori, F. Wilsch and K. Yamamoto, Flavour symmetries in the SMEFT, JHEP 08 (2020) 166 [arXiv:2005.05366] [INSPIRE].

[67] L.J. Hall and L. Randall, Weak scale effective supersymmetry, Phys. Rev. Lett. 65 (1990) 2939 [INSPIRE].

[68] G. D'Ambrosio, G.F. Giudice, G. Isidori and A. Strumia, Minimal flavor violation: An Effective field theory approach, Nucl. Phys. B 645 (2002) 155 [hep-ph/0207036] [InSPIRE].

[69] C. Jarlskog, Commutator of the Quark Mass Matrices in the Standard Electroweak Model and a Measure of Maximal CP-violation, Phys. Rev. Lett. 55 (1985) 1039 [INSPIRE].

[70] C. Jarlskog, A Basis Independent Formulation of the Connection Between Quark Mass Matrices, CP-violation and Experiment, Z. Phys. C 29 (1985) 491 [INSPIRE].

[71] A.L. Kagan, G. Perez, T. Volansky and J. Zupan, General Minimal Flavor Violation, Phys. Rev. D 80 (2009) 076002 [arXiv:0903.1794] [INSPIRE].

[72] R. Barbieri, G. Isidori, J. Jones-Perez, P. Lodone and D.M. Straub, U(2) and Minimal Flavour Violation in Supersymmetry, Eur. Phys. J. C 71 (2011) 1725 [arXiv:1105.2296] [INSPIRE]. 
[73] R. Barbieri, D. Buttazzo, F. Sala and D.M. Straub, Flavour physics from an approximate $\mathrm{U}(2)^{3}$ symmetry, JHEP 07 (2012) 181 [arXiv:1203.4218] [INSPIRE].

[74] G. Blankenburg, G. Isidori and J. Jones-Perez, Neutrino Masses and LFV from Minimal Breaking of $\mathrm{U}(3)^{5}$ and $\mathrm{U}(2)^{5}$ flavor Symmetries, Eur. Phys. J. C 72 (2012) 2126 [arXiv: 1204.0688] [INSPIRE].

[75] M. Bordone, O. Catà and T. Feldmann, Effective Theory Approach to New Physics with Flavour: General Framework and a Leptoquark Example, JHEP 01 (2020) 067 [arXiv: 1910.02641] [INSPIRE].

[76] L. Berthier and M. Trott, Consistent constraints on the Standard Model Effective Field Theory, JHEP 02 (2016) 069 [arXiv: 1508.05060] [INSPIRE].

[77] S. Dawson, P.P. Giardino and A. Ismail, Standard model EFT and the Drell-Yan process at high energy, Phys. Rev. D 99 (2019) 035044 [arXiv:1811.12260] [inSPIRE].

[78] C. Hartmann, W. Shepherd and M. Trott, The $Z$ decay width in the SMEFT: $y_{t}$ and $\lambda$ corrections at one loop, JHEP 03 (2017) 060 [arXiv:1611.09879] [INSPIRE].

[79] J.M. Cullen and B.D. Pecjak, Higgs decay to fermion pairs at NLO in SMEFT, JHEP 11 (2020) 079 [arXiv: 2007.15238] [inSPIRE].

[80] J. Baglio, S. Dawson and I.M. Lewis, NLO effects in EFT fits to $W^{+} W^{-}$production at the LHC, Phys. Rev. D 99 (2019) 035029 [arXiv:1812.00214] [INSPIRE].

[81] S. Descotes-Genon, A. Falkowski, M. Fedele, M. González-Alonso and J. Virto, The CKM parameters in the SMEFT, JHEP 05 (2019) 172 [arXiv:1812.08163] [INSPIRE].

[82] Particle Data Group collaboration, Review of Particle Physics, Prog. Theor. Exp. Phys. 2020 (2020) 083C01.

[83] S. Carrazza, C. Degrande, S. Iranipour, J. Rojo and M. Ubiali, Can New Physics hide inside the proton?, Phys. Rev. Lett. 123 (2019) 132001 [arXiv: 1905. 05215] [InSPIRE].

[84] J.R. Ellis, M.K. Gaillard and D.V. Nanopoulos, A Phenomenological Profile of the Higgs Boson, Nucl. Phys. B 106 (1976) 292 [InSPIRE].

[85] M.A. Shifman, A.I. Vainshtein, M.B. Voloshin and V.I. Zakharov, Low-Energy Theorems for Higgs Boson Couplings to Photons, Sov. J. Nucl. Phys. 30 (1979) 711 [INSPIRE].

[86] L. Bergstrom and G. Hulth, Induced Higgs Couplings to Neutral Bosons in $e^{+} e^{-}$Collisions, Nucl. Phys. B 259 (1985) 137 [Erratum ibid. 276 (1986) 744] [inSPIRE].

[87] A.V. Manohar and M.B. Wise, Modifications to the properties of the Higgs boson, Phys. Lett. B 636 (2006) 107 [hep-ph/0601212] [INSPIRE].

[88] D. Neill, Two-Loop Matching onto Dimension Eight Operators in the Higgs-Glue Sector, arXiv:0908.1573 [INSPIRE].

[89] R.V. Harlander and T. Neumann, Probing the nature of the Higgs-gluon coupling, Phys. Rev. D 88 (2013) 074015 [arXiv: 1308.2225] [INSPIRE].

[90] S. Dawson, I.M. Lewis and M. Zeng, Effective field theory for Higgs boson plus jet production, Phys. Rev. D 90 (2014) 093007 [arXiv:1409.6299] [INSPIRE].

[91] R. Frederix, S. Frixione, V. Hirschi, F. Maltoni, R. Pittau and P. Torrielli, Four-lepton production at hadron colliders: aMC@NLO predictions with theoretical uncertainties, JHEP 02 (2012) 099 [arXiv: 1110.4738] [INSPIRE]. 
[92] U. Baur and E.W.N. Glover, Higgs Boson Production at Large Transverse Momentum in Hadronic Collisions, Nucl. Phys. B 339 (1990) 38 [inSPIRE].

[93] V. Del Duca, W. Kilgore, C. Oleari, C.R. Schmidt and D. Zeppenfeld, Kinematical limits on Higgs boson production via gluon fusion in association with jets, Phys. Rev. D 67 (2003) 073003 [hep-ph/0301013] [INSPIRE].

[94] W.-Y. Keung and F.J. Petriello, Electroweak and finite quark-mass effects on the Higgs boson transverse momentum distribution, Phys. Rev. D 80 (2009) 013007 [arXiv:0905.2775] [INSPIRE].

[95] M. Buschmann, D. Goncalves, S. Kuttimalai, M. Schonherr, F. Krauss and T. Plehn, Mass Effects in the Higgs-Gluon Coupling: Boosted vs Off-Shell Production, JHEP 02 (2015) 038 [arXiv: 1410.5806] [INSPIRE].

[96] J.A. Gracey, Classification and one loop renormalization of dimension-six and dimension-eight operators in quantum gluodynamics, Nucl. Phys. B 634 (2002) 192 [Erratum ibid. 696 (2004) 295] [hep-ph/0204266] [INSPIRE].

[97] S. Dawson, Radiative corrections to Higgs boson production, Nucl. Phys. B 359 (1991) 283 [INSPIRE].

[98] L. Berthier, M. Bjørn and M. Trott, Incorporating doubly resonant $W^{ \pm}$data in a global fit of SMEFT parameters to lift flat directions, JHEP 09 (2016) 157 [arXiv:1606.06693] [INSPIRE].

[99] M.J.G. Veltman, Unitarity and causality in a renormalizable field theory with unstable particles, Physica 29 (1963) 186 [INSPIRE].

[100] R.G. Stuart, Gauge invariance, analyticity and physical observables at the Z0 resonance, Phys. Lett. B 262 (1991) 113 [inSPIRE].

[101] M.W. Grunewald et al., Reports of the Working Groups on Precision Calculations for LEP2 Physics: Proceedings. Four fermion production in electron positron collisions, hep-ph/0005309 [INSPIRE].

[102] R. Gauld, B.D. Pecjak and D.J. Scott, QCD radiative corrections for $h \rightarrow b \bar{b}$ in the Standard Model Dimension-6 EFT, Phys. Rev. D 94 (2016) 074045 [arXiv: 1607.06354] [INSPIRE].

[103] G. Brooijmans et al., Les Houches 2017: Physics at TeV Colliders New Physics Working Group Report, in 10th Les Houches Workshop on Physics at TeV Colliders, (2018) [arXiv: 1803.10379] [INSPIRE].

[104] N.D. Christensen et al., Simulating spin- $\frac{3}{2}$ particles at colliders, Eur. Phys. J. C 73 (2013) 2580 [arXiv: 1308.1668] [INSPIRE].

[105] J. Gao, C.S. Li and H.X. Zhu, Top Quark Decay at Next-to-Next-to Leading Order in QCD, Phys. Rev. Lett. 110 (2013) 042001 [arXiv:1210.2808] [INSPIRE].

[106] J. Alwall, C. Duhr, B. Fuks, O. Mattelaer, D.G. Öztürk and C.-H. Shen, Computing decay rates for new physics theories with FeynRules and MadGraph 5_aMC@NLO, Comput. Phys. Commun. 197 (2015) 312 [arXiv:1402.1178] [INSPIRE].

[107] LHC Higgs Cross Section Working Group collaboration, SM Higgs Branching Ratios and Total Decay Widths, https://twiki.cern.ch/twiki/bin/view/LHCPhysics/CERNYellowReportPageBR. 
[108] A. Bredenstein, A. Denner, S. Dittmaier and M.M. Weber, Precise predictions for the Higgs-boson decay $H \rightarrow W W / Z Z \rightarrow 4$ leptons, Phys. Rev. D 74 (2006) 013004 [hep-ph/0604011] [INSPIRE].

[109] Wolfram Research, Inc., Mathematica, Version 12.1, Champaign, IL (2020).

[110] https://launchpad.net/mg5amcnlo.

[111] http://amcatnlo.web.cern.ch/amcatnlo/list_refs.htm.

[112] C. Hays, A. Helset, A. Martin and M. Trott, Exact SMEFT formulation and expansion to $\mathcal{O}\left(v^{4} / \Lambda^{4}\right)$, JHEP 11 (2020) 087 [arXiv: 2007.00565] [INSPIRE].

[113] LHC Higgs Cross Section Working Group collaboration, Handbook of LHC Higgs Cross Sections: 4. Deciphering the Nature of the Higgs Sector, arXiv:1610.07922 [INSPIRE].

[114] J.R. Andersen et al., Les Houches 2015: Physics at TeV Colliders Standard Model Working Group Report, in 9th Les Houches Workshop on Physics at TeV Colliders, (2016) [arXiv: 1605.04692] [INSPIRE].

[115] N. Berger et al., Simplified Template Cross Sections - Stage 1.1, arXiv:1906.02754 [INSPIRE].

[116] S. Amoroso et al., Les Houches 2019: Physics at TeV Colliders: Standard Model Working Group Report, in 11th Les Houches Workshop on Physics at TeV Colliders: PhysTeV Les Houches, (2020) [arXiv:2003.01700] [INSPIRE].

[117] O. Mattelaer, On the maximal use of Monte Carlo samples: re-weighting events at NLO accuracy, Eur. Phys. J. C $\mathbf{7 6}$ (2016) 674 [arXiv:1607.00763] [INSPIRE].

[118] P.J. Mohr, B.N. Taylor and D.B. Newell, CODATA Recommended Values of the Fundamental Physical Constants: 2010, Rev. Mod. Phys. 84 (2012) 1527 [arXiv:1203.5425] [INSPIRE].

[119] CDF and D0 collaborations, Combination of CDF and Do W-Boson Mass Measurements, Phys. Rev. D $8 \mathbf{8}$ (2013) 052018 [arXiv:1307.7627] [INSPIRE].

[120] ALEPH, DELPHI, L3, OPAL and SLD collaboration, LEP Electroweak Working Group, SLD Electroweak Group and SLD Heavy Flavour Group, Precision electroweak measurements on the $Z$ resonance, Phys. Rept. 427 (2006) 257 [hep-ex/0509008] [InSPIRE].

[121] http://feynrules.irmp.ucl.ac.be/wiki/dim6top.

[122] http://feynrules.irmp.ucl.ac.be/wiki/SMEFTatNLO.

[123] code.launchpad.net/ ${ }^{\sim}$ rwgtdim6/mg5amcnlo/plugin_eft_contrib. 